\title{
Probes of pulsar emission physics: The double pulsar and the gamma-ray pulsar population
}

\author{
Benetge Bhakthi Pranama Perera \\ West Virginia University
}

Follow this and additional works at: https://researchrepository.wvu.edu/etd

\section{Recommended Citation}

Perera, Benetge Bhakthi Pranama, "Probes of pulsar emission physics: The double pulsar and the gammaray pulsar population" (2013). Graduate Theses, Dissertations, and Problem Reports. 233.

https://researchrepository.wvu.edu/etd/233

This Dissertation is protected by copyright and/or related rights. It has been brought to you by the The Research Repository @ WVU with permission from the rights-holder(s). You are free to use this Dissertation in any way that is permitted by the copyright and related rights legislation that applies to your use. For other uses you must obtain permission from the rights-holder(s) directly, unless additional rights are indicated by a Creative Commons license in the record and/ or on the work itself. This Dissertation has been accepted for inclusion in WVU Graduate Theses, Dissertations, and Problem Reports collection by an authorized administrator of The Research Repository @ WVU.

For more information, please contact researchrepository@mail.wvu.edu. 


\title{
PROBES OF PULSAR EMISSION PHYSICS: THE DOUBLE PULSAR AND THE GAMMA-RAY PULSAR POPULATION
}

\author{
by \\ Benetge Bhakthi Pranama Perera
Dissertation submitted to the Eberly College of Arts and Sciences at West Virginia University in partial fulfillment of the requirements for the degree of
Doctor of Philosophy in Physics

Dr. Maura McLaughlin, Ph.D., Chair

Dr. Duncan Lorimer, Ph.D.

Dr. D.J. Pisano, Ph.D.

Dr. Paul Cassak, Ph.D.

Dr. Arun Ross, Ph.D.

Department of Physics

Morgantown, West Virginia 2013 


\title{
ABSTRACT \\ Probes of Pulsar Emission Physics: The Double Pulsar and the Gamma-Ray Pulsar Population
}

\author{
Benetge Bhakthi Pranama Perera
}

The double pulsar, PSR J0737-3039A/B, is a unique binary system in which both neutron stars have been detected as radio pulsars. We analyze the evolution of the radio emission from the second born, $2.8 \mathrm{~s}$ pulsar (pulsar B) based on five years of Green Bank Telescope data since 2003 December. We find that the pulse profile and the flux density of pulsar B change significantly over time, culminating in its radio emission disappearance towards our line of sight since 2008 March. Over this time, the flux density decreases dramatically and the pulse profile evolves from a single to a double peak. This profile shape evolution is likely caused by relativistic spin precession. We explain the profile evolution by an elliptical beam shape geometry model based on geodetic spin precession. By fitting for the observed pulse profile widths, the model constrains the geometry angles of pulsar B, namely the magnetic misalignment from the spin axis $\alpha_{\mathrm{B}}=61^{\circ}$ and the spin misalignment from the orbit normal $\theta_{\mathrm{B}}=138.5^{\circ}$, which are consistent with and similar to those derived by Breton et al. (2008) with a completely different geometry framework. The elliptical beam model predicts that the radio emission reappearance from pulsar B towards our line of sight is expected to happen between 2014 and 2035, with the variation depending on assumptions of the symmetry of the beam.

The strong stellar wind produced by the high spin-down luminosity of the first born, recycled, $23 \mathrm{~ms}$ pulsar (pulsar A) of the double pulsar system distorts the magnetosphere of its companion pulsar B. The wind-magnetosphere interaction model determines a bow-shock around pulsar B and it is likely the boundary of its magnetosphere. With geodetic spin precession, pulsar B provides an excellent opportunity to study different emission regions in the magnetosphere. Using the distorted magnetosphere and the well-defined geometrical parameters of pulsar B, we estimate the emission altitude to be $\sim 20$ neutron star radii in the bright orbital longitude regions. We further find that the emission altitude varies across the orbit due to the change in the orientation of the bow-shock with respect to our line of sight. Moreover, the emission altitude of pulsar B changes over time due to spin precession.

We then study the pulse profile variation of pulsar A. Analyzing more than six years of data, we confirm that pulsar A does not show a significant pulse width variation over time, which is consistent with previous works. Following a similar geometry framework as for pulsar B, we determine the geometry of pulsar A based 
on geodetic spin precession, including subtle changes of the pulse width at lower intensity levels from pulse peaks. Using a simple double-pole circular beam model, we find that pulsar $\mathrm{A}$ is nearly orthogonal, i.e., $\alpha_{\mathrm{A}}=88.1^{\circ}$ and $\theta_{\mathrm{A}}=0.9^{\circ}$, which is consistent with the results of Ferdman et al. (2013). We then use an advanced magnetosphere model including vacuum retarded dipole configuration with a polar cap geometry to synthesize pulse profiles of pulsar A. By fitting the modeled profiles to observed profiles, we constrain the physical properties of its radio beam, namely the beam size $\rho_{\mathrm{A}} \approx 30^{\circ}$ and emission altitude $\sim 10$ neutron star radii. By knowing the complete geometry of both pulsars, we construct the full geometrical configuration of the system. We find that the relative angle between the spin axes of the two pulsars varies periodically over time. This is the first time that this relative spin angle has been estimated for a double neutron star system.

Finally, we use Fermi Gamma-ray Space Telescope results on non-recycled pulsars to study the gamma-ray pulsar population. We use pulsar detections obtained from the Large Area Telescope (LAT) to constrain how the gamma-ray luminosity $L_{\gamma}$ depends on the period $P$ and the period derivative $\dot{P}$. An outer gap (OG) magnetosphere geometry provides the best-fit model, which is $L_{\gamma} \propto P^{-a} \dot{P}^{b}$ where $a=1.36 \pm 0.03$ and $b=0.44 \pm 0.02$, similar to but not identical to the commonly assumed $L_{\gamma} \propto \sqrt{\dot{E}} \propto P^{-1.5} \dot{P}^{0.5}$. Given upper limits on gamma-ray fluxes of currently known radio pulsars and using the OG model, we find that about $92 \%$ of the radio-detected pulsars have gamma-ray beams that intersect our line of sight. By modeling the misalignment of radio and gamma-ray beams of these pulsars, we find an average gamma-ray beaming solid angle of about $3.7 \pi$ for the OG model, assuming a uniform beam. Using LAT-measured diffuse fluxes, we place a $2 \sigma$ upper limit on the average braking index and a $2 \sigma$ lower limit on the average surface magnetic field strength of the gamma-ray pulsar population of 3.8 and $3.2 \times 10^{10} \mathrm{G}$, respectively. We then predict the number of non-recycled pulsars detectable by the LAT based on our population model. Using the two-year sensitivity, we find that the LAT is capable of detecting emission from about 380 non-recycled pulsars, including 150 currently identified radio pulsars. Using the expected five-year sensitivity, about 620 non-recycled pulsars are detectable, including about 220 currently identified radio pulsars. We note that these predictions are significantly dependent on our model assumptions. 


\section{Acknowledgments}

There are many people that I would like to thank for their great support on this work in numerous ways. Without their help, I would not be able to come this far and survive in this field. Thus, I would like to mention them at this point.

First of all, I must thank Dr. Maura McLaughlin for supervising me on all of these research projects and helping me throughout this path. She generously shared her knowledge and I learned most of the pulsar astronomy I know today from her. Most importantly, I learned how to tackle and solve problems by talking to her when I got stuck on a research problem. This really helped me to go through this path smoothly.

Next I must thank my other committee members, Dr. Duncan Lorimer, Dr. D.J. Pissano, Dr. Paul Cassak, and Dr. Arun Ross for reviewing this work and providing valuable comments. Specially I want to thank Dunc for accepting me to the WVU pulsar group even though I did not have any astronomy background at that time. Also I learned lot of astronomy from his wonderful classes.

I would like to thank Dr. Maxim Lyutikov for giving me the opportunity to come and work at Purdue University with David and Kostas on the double pulsar modeling. Also I must thank all the other collaborators for sharing and reviewing my work.

I would also like to thank the WVU Physics department, Dr. Earl Scime, for giving me this opportunity to study as a graduate student at WVU. Also I would like to mention Sherry, Greg, Siobhan, Val, Audrey, and Lori for their great support on various occasions. Also, I would like to mention postdocs Vlad, Chunglee, and Manjari for their valuable help on computing and other things.

Without my fellow graduate students, this life would have been hard, so I would like to thank all of them. Also I would like to mention my office mates Jason, Josh R, Josh M, Mitch, and Priya in Hodges hall, and Jayanth and Nipuni in White hall. You all helped me in many ways. Specially, I would like to thank all of my Sri Lankan friends in Morgantown. The time I spent with you all made my stay in Morgantown enjoyable. All the discussions we had were wonderful and will be cherished forever.

Finally, a big thank must go to my wonderful parents, Amma and Thaththa, for showing my the beauty of this world. Then I must thank my brother and his family - ayya, akka, Duvish, and Vidush - for their great support. I must specially mention my grand parent, Seeya, for directing me into education when I was a kid. Without you all, I would not have been able to achieve this. 


\section{Table of Contents}

List of Tables $\quad$ vii

List of Figures viii

List of Abbreviations and Symbols $\quad x$

1 Introduction 1

1.1 A brief history of the discovery of pulsars . . . . . . . . . . . . . 1

1.2 Properties and theoretical background of pulsars . . . . . . . . . . 3

1.2.1 Mass, radius, and moment of inertia of NSs . . . . . . . 6

1.2.2 Rotational kinetic energy . . . . . . . . . . . . . . . 8

1.2.3 Magnetic field strength . . . . . . . . . . . . . . . . . . 9

1.2.4 Age estimate . . . . . . . . . . . . . . . . . . 10

1.2.5 $P-\dot{P}$ diagram of pulsars . . . . . . . . . . . . . . 12

1.2.6 Pulsar distances . . . . . . . . . . . . . . . . . . . 14

1.2.6.1 Parallax measurements . . . . . . . . . . . . 14

1.2.6.2 HI measurements . . . . . . . . . . . . . . . . 15

1.2.6.3 DM-derived distances . . . . . . . . . . . 15

1.3 The pulsar magnetosphere . . . . . . . . . . . . . . . . 17

1.3.1 Pulsar radio emission . . . . . . . . . . . . . . . 20

1.3.2 Pulsar gamma-ray emission . . . . . . . . . . . . . 25

1.4 Pulsar geometry with polarization studies and the rotating vector model 29

1.5 Pulsar radio beams and emission altitudes . . . . . . . . . . . . 32

1.6 Pulsar timing . . . . . . . . . . . . . . . . . . . . . . . . . . . 35

1.7 Pulsars in binary systems . . . . . . . . . . . . . . 36

1.7.1 Timing binary pulsars . . . . . . . . . . . . . . 37

1.7.2 Relativistic spin precession and radio emission variability in DNSs ....................... 39

1.8 Double pulsar system PSR J0737-3039A/B . . . . . . . . . . . 43

2 Radio emission evolution and the geometry of J0737-3039B 52

2.1 Introduction . . . . . . . . . . . . . . . . . . . 52

2.2 Observations . . . . . . . . . . . . . . . . . 53

2.2.1 Pulse profile evolution of the two bright phases . . . . . . . 54

2.2.2 Flux evolution of the two bright phases . . . . . . . . . . . . 58

2.2.3 Analysis and comparison of light curves of the two bright phases 61

2.2.4 Analysis of the two weak phases . . . . . . . . . . . . 64

2.3 Determining the geometry of pulsar B with geodetic spin precession . 66

2.3.1 Modeling the geometry with a circular hollow-cone beam . . . 67

2.3.2 Modeling the geometry with a 2-dimensional elliptical horseshoe shaped beam . . . . . . . . . . . . . . 71

2.3.3 Revisiting the geometry with a 3-dimensional elliptical beam model ................... . . 78 
2.4 Discussion . . . . . . . . . . . . . . . . . . . 85

3 The emission altitudes of J0737-3039B 88

3.1 Introduction . . . . . . . . . . . . . . . . . . . . . . . . 88

3.2 Boundary model . . . . . . . . . . . . . . . . . . . . . . . . . . . . . . . . . . . 90

3.3 Tracing the dipole field lines . . . . . . . . . . . . . . . . . . . . 97

3.4 Emission height calculation . . . . . . . . . . . . . . . 103

3.5 Discussion . . . . . . . . . . . . . . . . . . . . . 110

4 The geometry of PSR J0737-3039A and its radio beam 115

4.1 Introduction . . . . . . . . . . . . . . . . . . . 115

4.2 Pulse Profiles of Pulsar A . . . . . . . . . . . . . . . . 117

4.3 Geometry of pulsar A with a simple circular beam . . . . . . . . . . . 119

4.4 Geometry of pulsar A with a retarded vacuum dipole PC model . . . 122

4.4.1 Fitting Pulse Profiles of pulsar A . . . . . . . . . . . 130

4.4.2 Results........................ 132

4.5 The 3-D Orbital Geometry of the Double Pulsar . . . . . . . . . . . . 134

4.6 Discussion . . . . . . . . . . . . . . . . . . . 137

5 Modeling the non-recycled Fermi gamma-ray pulsar population $\quad 140$

5.1 Introduction . . . . . . . . . . . . . . . . 140

5.2 Data . . . . . . . . . . . . . . . . . . . . 144

5.2.1 Pulsed gamma-ray detections from GRPs . . . . . . . . . . 144

5.2.2 Gamma-ray flux upper limits . . . . . . . . . . . . . . 147

5.2.3 Gamma-ray diffuse flux measurements . . . . . . . . . . . . . 148

5.3 Luminosity law . . . . . . . . . . . . . . . . . . . . . . . . 149

5.4 Luminosity law likelihood analysis . . . . . . . . . . . . . . . . 150

5.5 Luminosity law analysis and results . . . . . . . . . . . . . . . 152

5.5.1 Constant beaming factor . . . . . . . . . . . . . . . 153

5.5.2 Period-dependent beaming factor . . . . . . . . . . . . 158

5.5.3 Phase-shift dependent beaming factor . . . . . . . . . . . . . . 159

5.5.4 Outer magnetosphere model dependent beaming factor . . . . 160

5.6 Determining the geometry of gamma-ray and radio beams . . . . . 163

5.7 Properties and population analysis of GRPs with diffuse flux measurements . . . . . . . . . . . . . . . . . 170

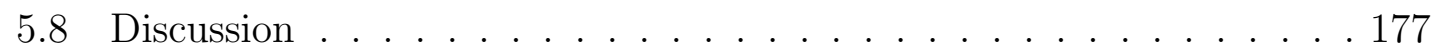

6 Conclusions and Future work 180

6.1 PSR J0737-3039A/B and future work related with the system . . . . 180

6.2 Fermi gamma-ray analysis and future work . . . . . . . . . . . . . 182

A Derivation of the elliptical beam width corresponding to CW08 192

B Derivation of the new elliptical beam 194

$\begin{array}{ll}\text { C Gamma-ray pulsar population } & 197\end{array}$ 


\section{List of Tables}

1.1 Properties of DNS systems . . . . . . . . . . . . . . . . . . . 42

1.2 Starting and ending orbital longitudes of each phase of pulsar B . . . 48

2.1 The derived geometry parameters of pulsar B . . . . . . . . . . . 71

4.1 Geometric parameters of known pulsar binaries . . . . . . . . . 123

5.1 Fermi gamma-ray pulsar detections . . . . . . . . . . . . . . . . 145

5.2 Gamma-ray pulsar luminosity law . . . . . . . . . . . . . . . . . . 154

5.3 Estimated geometries of gamma-ray pulsars . . . . . . . . . . . 162 


\section{List of Figures}

1.1 Discovery plot of the first pulsar CP 1919 . . . . . . . . . . . . 2

1.2 Schematic view of a pulsar magnetosphere . . . . . . . . . . . 5

1.3 Integrated pulse profiles of pulsars . . . . . . . . . . . . . 6

1.4 Neutron star masses of binary systems . . . . . . . . . . . . . . 7

$1.5 P-\dot{P}$ diagram of pulsars . . . . . . . . . . . . . . . . . . . . . . . . . . 13

1.6 Pulsar magnetosphere . . . . . . . . . . . . . . . . . . 19

1.7 Pair cascade in the pulsar magnetosphere . . . . . . . . . . . . 23

1.8 Outer gap emission model . . . . . . . . . . . . . . . . . . 26

1.9 Polarization profiles and PPA swing . . . . . . . . . . . . . 30

1.10 Core and conal beam structure and patchy beam . . . . . . . . . 33

1.11 Orbital decay of PSR B1913+16 . . . . . . . . . . . . . . . . . 40

1.12 Modulation in pulsar A's radio emission due to pulsar B . . . . . . . 45

1.13 The schematic diagram of the pulsar geometry and the two orbits of PSR J0737-3039A/B . . . . . . . . . . . . . . . . . 46

1.14 The observed radio emission of PSR J0737-3039B . . . . . . . . . . . 49

1.15 Drifting sub-pulses of J0737-3039B . . . . . . . . . . . . . 50

1.16 Mass-mass diagram of J0737-3039A/B . . . . . . . . . . . . . 51

2.1 Pulse profiles of BP1 of pulsar B . . . . . . . . . . . . . 55

2.2 Pulse profiles of BP2 of pulsar B . . . . . . . . . . . . 56

2.3 Grey-scale plot of two BPs . . . . . . . . . . . . . . 57

2.4 FWHM values of BP1 . . . . . . . . . . . . . . . . . . 57

2.5 FWHM values of BP2 . . . . . . . . . . . . . . 58

2.6 Separation of the two peaks over time . . . . . . . . . . . . 59

2.7 Mean flux density of BPs over time . . . . . . . . . . . . . . . 60

2.8 Light curves of BP1 of pulsar B . . . . . . . . . . . . . . . 62

2.9 Light curves of BP2 of pulsar B . . . . . . . . . . . . . . . 63

2.10 Evolution of the two BPs . . . . . . . . . . . . . . 63

2.11 Pulse profiles of WP1 . . . . . . . . . . . . . . . 65

2.12 Pulse profiles of WP2 . . . . . . . . . . . . . . . . . . . 65

2.13 Mean flux density of WPs over time . . . . . . . . . . . . 66

2.14 Geometry of the coordinate system of pulsar B . . . . . . . . . . . 69

2.15 Best-fit of the circular hollow-cone beam model . . . . . . . . . . . 70

2.16 Schematic view of the elliptical model . . . . . . . . . . . . . . 72

2.17 Posterior probabilities of the likelihood analysis . . . . . . . . . 75

2.18 Best-fit plot of the elliptical hollow cone beam model . . . . . . . . . 76

2.19 High-resolution mean pulse profiles of pulsar B . . . . . . . . . . . 79

2.20 The geometry of the elliptical emission cone . . . . . . . . . . . . . . 82

2.21 Result of the new elliptical hollow cone beam model . . . . . . . . . . 83

3.1 Schematic geometry of the wind-magnetospheric interaction model . . 92

3.2 Cartesian coordinate system of the geometry of pulsar B . . . . . . . 99 
3.3 Confined magnetosphere from the wind-magnetospheric interaction model . . . . . . . . . . . . . . . . . . . . . . . 102

3.4 Emission height variation across a single orbit . . . . . . . . . 108

3.5 Emission height variation of BP1 over time . . . . . . . . . . . 109

3.6 Emission height variation of BP2 over time . . . . . . . . . . . 110

4.1 Integrated pulse profile of PSR J0737-3039A . . . . . . . . . . 118

4.2 Pulse profile widths at $5 \%$ intensity of pulsar A . . . . . . . . . 119

4.3 Retarded vacuum dipole magnetosphere . . . . . . . . . . 126

4.4 Photon map in the sky from the last closed field lines . . . . . . . . 128

4.5 Schematic diagram of the radio emission beam . . . . . . . . . . . 129

4.6 Model pulse profiles of A with geometries from M1 and M2 . . . 133

4.7 Model pulse profiles of A with geometries from OG, TPC, and RVM . 134

4.8 The variation of the relative angle between the spin axes of the $\mathrm{A}$ and B pulsars . . . . . . . . . . . . . . . 136

5.1 Marginalized PDFs for luminosity parameters . . . . . . . . . . 155

5.2 Model-estimated fluxes for LAT-detected non-recycled pulsars with

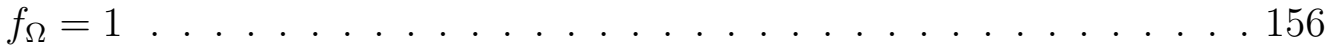

5.3 Model-estimated luminosity vs. spin-down luminosity for LAT-detected pulsars . . . . . . . . . . . . . . . . . . . . 157

5.4 Model-estimated fluxes for LAT-detected non-recycled pulsars with TPC model . . . . . . . . . . . . . . . . . . . . . . . . . . 164

5.5 Model-estimated fluxes for LAT-detected non-recycled pulsars with OG model . . . . . . . . . . . . . . . . . . . . . . . . . 165

5.6 Model-estimated fluxes of non-recycled radio pulsars . . . . . . . 168

5.7 Marginalized PDFs for $n, B, P_{0, \min }$, and $P_{0, \max } \ldots \ldots \ldots \ldots$

5.8 The fraction of diffuse flux due to pulsars as a function of $n$ and $B \quad .176$

5.9 Predicted Fermi LAT energy fluxes of the GRPs . . . . . . . . . 176

5.10 The luminosity distribution of GRPs . . . . . . . . . . . 177

A.1 Projected trajectories of the elliptical beam . . . . . . . . 193

B.1 Schematic view of the new elliptical model . . . . . . . . . . . . 195 


\section{List of Abbreviations and Symbols}

\begin{tabular}{|c|c|}
\hline NS & Neutron star \\
\hline GR & General relativity \\
\hline GW & Gravitational wave \\
\hline $\mathrm{CP}$ & Canonical (or normal) pulsar \\
\hline MSP & Millisecond pulsar \\
\hline DNS & Double neutron star \\
\hline $\mathrm{DM}$ & Dispersion measure \\
\hline $\mathrm{CR}$ & Curvature radiation \\
\hline $\mathrm{PC}$ & Polar cap \\
\hline OG & Outer gap \\
\hline SG & Slot gap \\
\hline $\mathrm{TPC}$ & Two-pole caustic \\
\hline RVM & Rotating vector model \\
\hline PPA & Linear polarization position angle \\
\hline TOA & Time of arrival \\
\hline GRP & Gamma-ray pulsar \\
\hline GBT & Green Bank Telescope \\
\hline Fermi & Fermi Gamma-ray Telescope \\
\hline LAT & Large Area Telescope \\
\hline$M_{\odot}$ & Solar mass \\
\hline$d_{\odot}$ & Distance to the Sun from the Galactic center \\
\hline$l$ & Galactic longitude \\
\hline$b$ & Galactic latitude \\
\hline $\mathrm{R}_{\mathrm{NS}}$ & Neutron star radius $(=10 \mathrm{~km})$ \\
\hline$c$ & Speed of light \\
\hline$m$ & Magnetic moment $(=|\vec{m}|)$ \\
\hline$I$ & Neutron star moment of inertial $\left(=10^{45} \mathrm{~g} \mathrm{~cm}^{2}\right)$ \\
\hline $\mathrm{R}_{\mathrm{LC}}$ & Light cylinder radius \\
\hline$D$ & Distance to the pulsar \\
\hline$\Omega$ & Rotational angular frequency \\
\hline $\mathrm{P}$ & Spin period \\
\hline$\dot{P}$ & Derivative of the spin period \\
\hline$n$ & Braking index \\
\hline$B_{12}$ & Magnetic field strength in $10^{12}$ Gauss \\
\hline$P_{0}$ & Initial spin period \\
\hline$\dot{P}_{15}$ & Derivative of the spin period in $10^{-15}$ \\
\hline$\dot{E}$ & Spin-down luminosity \\
\hline$L_{\gamma}$ & Gamma-ray pulsar luminosity \\
\hline$G$ & Gamma-ray pulsar energy flux \\
\hline
\end{tabular}




\section{List of Abbreviations and Symbols (Cont....)}

$\alpha \quad$ Misalignment of the magnetic axis from the spin axis

$\theta \quad$ Misalignment of the spin axis from the orbit normal

$\zeta \quad$ The angle between the spin axis and the line of sight

$\beta \quad$ Impact parameter (or the closest approach of the magnetic axis to the line of sight)

$f_{\Omega} \quad$ Beaming factor

$\Omega_{\gamma} \quad$ Beaming solid angle

$\Omega_{\gamma \mathrm{i}}$ Instantaneous gamma-ray beam solid angle

$\rho \quad$ Half-opening angle of the beam 


\section{Chapter 1}

\section{Introduction}

Pulsars are rapidly rotating highly magnetized neutron stars (NSs) which emit electromagnetic radiation. These are born from supernova explosions of massive stars and powered by loss of their rotational kinetic energy. These highly compact objects have densities which compare with that of an atomic nucleus. Pulsars are

ideal sources for understanding the physics of NSs due to emission of radiation from radio to gamma-ray wavelengths.

\subsection{A brief history of the discovery of pulsars}

Pulsars were first discovered in 1967 by Jocelyn Bell, a graduate student at University of Cambridge (Lyne \& Smith, 2004). She worked with her advisor, Dr. Antony Hewish, to construct a radio telescope to study quasars by using interplanetary scintillation. She recorded radio data on hundreds of meters of paper by using a chart recorder. These data included radio signals from space and also radio interference from the ground. On August 6, she found that there was a pulsing signal on the recorded paper, but Hewish believed that it was interference. However, the following observations showed that this signal appeared four minutes earlier every day, synchronized with the sidereal time. This convinced Hewish to conclude that the signal originated from an astronomical object. High-resolution follow-up obser- 


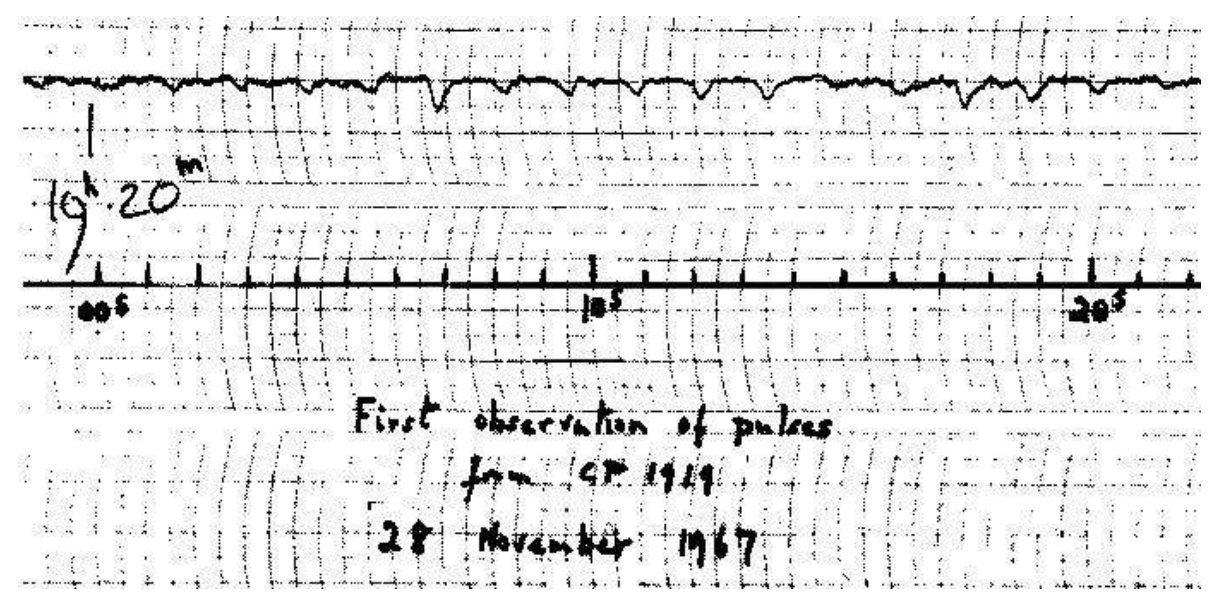

Figure 1.1: Discovery observation of the first pulsar LGM-1, or CP 1919 (now it is known as PSR J1921+2153).

vations showed that this object was emitting regular pulsations every 1.33 seconds (see Figure 1.1). The source was dubbed LGM-1 (Little Green Man 1), because it was thought that perhaps the cause of the signal was from an intelligent form of extraterrestrial life.

Soon after this discovery, she found another similar source at a different position in the sky. Follow-up observations further confirmed these two sources and identified two new sources, revealing that LGM-1 was not unique. Therefore, LGM1 was named as CP 1919 according its right ascension (19h 19m) and other three were named as CP 1133, CP 0834 and CP 0950. After confirming observations and data, they published the discovery of the new astronomical object CP 1919 on February 28, 1968 (Hewish et al., 1968). The second paper came out on April 13 reporting the discovery of the other three sources and further timing results of CP 1919 (Pilkington et al., 1968). Since the number of pulsar detections increased significantly with time, it required a proper method to identify them. In order to do that, pulsars are now named according to their position in the sky with right 
ascension and declination; i.e., CP 1919, CP 1133, CP 0834, and CP 0950 are named as PSRs J1921+2153, J1136+1551, J0837+0610, and J0953+0755, respectively.

Since then, over more than four decades, we have observed more than 2000 pulsars in the Galaxy and developed different kinds of theories to understand and explain the physics of these exotic astronomical objects. These sources are special tools for understanding basic theories in physics. They are also ideal sources for experiments which we cannot conduct in laboratories such as testing General Relativity (GR). In the near future, they will be key objects in direct gravitational wave detection through pulsar timing arrays.

\subsection{Properties and theoretical background of pulsars}

When a massive star $\left(\sim 8-25 \mathrm{M}_{\odot}\right)$ undergoes a core collapse supernova explosion at the end of its life cycle, a compact dense star (called a NS) is formed. This highly magnetized $\left(\sim 10^{12}\right.$ Gauss $)$ compact star has a small spin period $(\sim 0.001-$ 1 second) and almost all of its energy is released by loss of the rotational kinetic energy mainly through the ejection of relativistic particles and the emission of nonthermal electromagnetic radiation. The electromagnetic radiation (from radio to gamma-ray wavelengths) from the NS is observed as pulsations due to the NS spin. This active NS is called a pulsar if detectable given our line of sight.

In general, the NS magnetosphere can be understood as a magnetic dipole. Figure 1.2 shows a "toy model" of a pulsar magnetosphere, sometimes known as the "lighthouse model". The radio emission of pulsars originates within the inner 
acceleration gap and is centered around the magnetic axis. Thus, the radio emission is low-altitude, extending from the NS surface up to about a few hundred kilometers, and emitted as a narrow beam. In general, we assume pulsars are bipolar, having two radio beams, one for each magnetic pole. With a misaligned magnetic axis, we detect pulses from the pulsar when its radio beam crosses our line of sight while it is spinning, analogous to a lighthouse beam. On the other hand, the highenergy gamma-ray emission of pulsars originates at higher altitudes within outer acceleration gaps in the outer magnetosphere (see Figure 1.2). Therefore, gammaray pulsars have wider fan-like beams, covering a larger fraction of the sky compared to radio beams.

The individual radio pulses of pulsars are very weak signals and can only be seen from brighter sources. Therefore, we fold hundreds to thousands of single pulses on top of each other depending on the observation length and create a total pulse profile which is known as the "integrated pulse profile". Its vertical axis represents the intensity and the horizontal axis represents a spin rotation in degrees $\left(0^{\circ}-360^{\circ}\right)$, or spin phase. The shape of the pulse profile depends on the viewing geometry and the intrinsic beam structure of the pulsar, which is unique for the given source. For instance, if the magnetic misalignment of the pulsar is zero, we do not see pulses at all, but continuous radio emission only when our line of sight is within the radio emission beam. On the other hand, if the magnetic misalignment is close to $90^{\circ}$ and our line of sight is nearly perpendicular to the rotation axis, we observe radio emission from both poles of the pulsar, resulting in a main and an interpulse in the pulse profile with a separation of about $\sim 180^{\circ}$. In addition to the geometry, the 


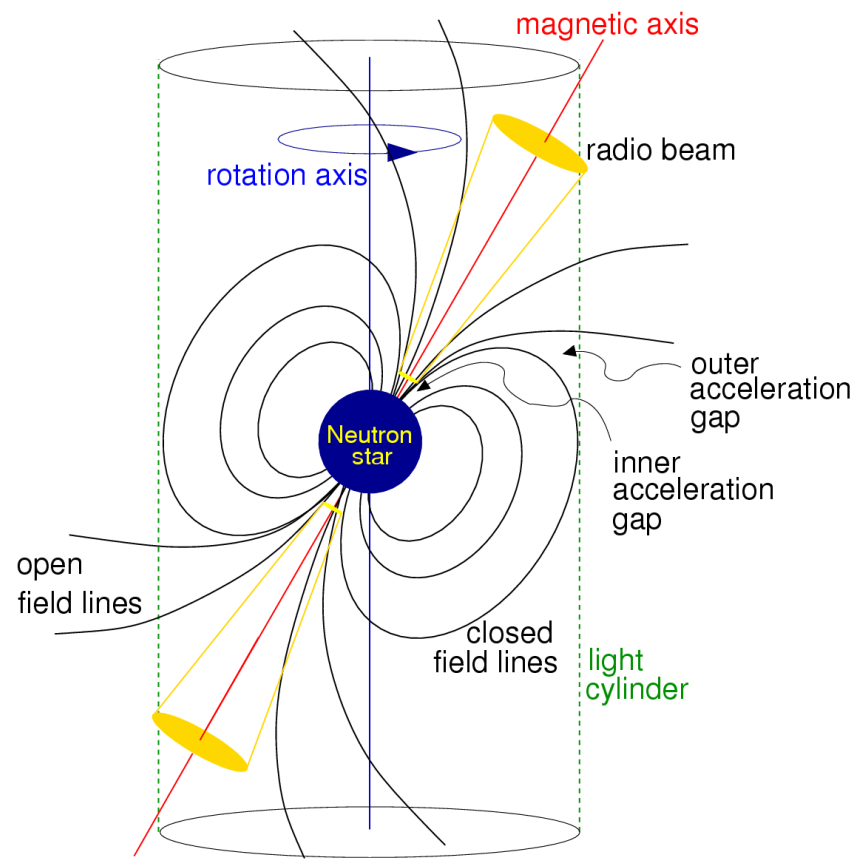

Figure 1.2: Lighthouse model of a rotating pulsar and its magnetosphere (Lorimer \& Kramer, 2005). The magnetic axis is misaligned with the vertical rotation axis. The closed field lines are defined with respect to the light cylinder, which is marked with dotted lines. The light cylinder is an imaginary boundary of the NS magnetosphere where the corotating particles obtain the maximum velocity, the speed of light. The field lines which are outside of this boundary are known as open field lines. The radio beams are located at each pole of the NS centered around the magnetic axis. 


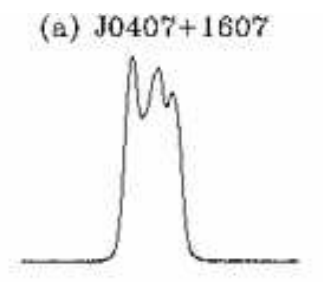

(d) $80826-34$

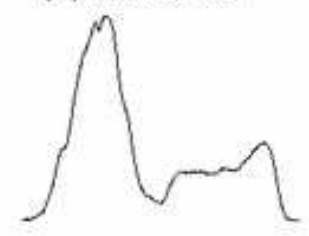

(g) $\mathrm{B} 1913+16$

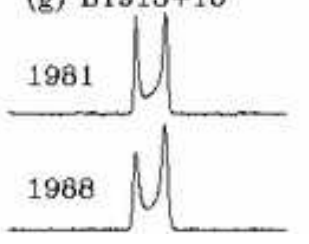

(b) $\mathrm{J} 0437-4715$
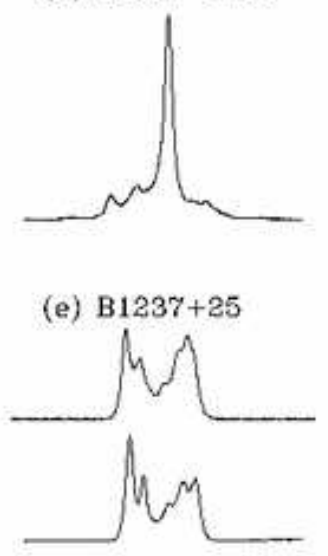

(h) $\mathrm{B} 1933+16$

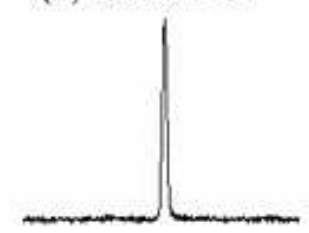

(c) J0737-3039A

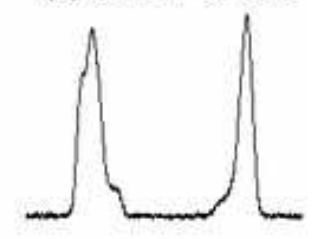

(f) $\mathrm{B} 1702-19$
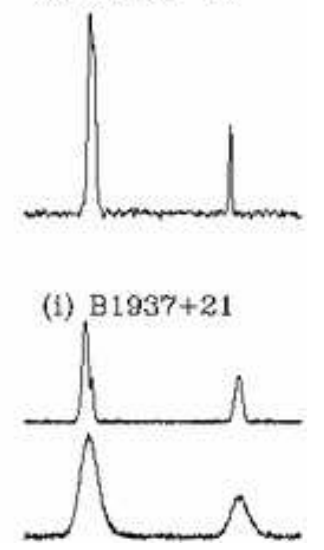

Figure 1.3: Integrated pulse profiles of nine pulsars (Lorimer \& Kramer, 2005). Each profile shows a full rotational phase except B1237+25. The profile of J0737-3039A shows the main pulse and the interpulse separated by about $180^{\circ}$. Two pulse profiles of B1913+16 are at two different epochs, showing profile evolution. This is discussed in Section 1.7.2. The two profiles of B1937+21 were obtained using two different data analysis techniques, namely coherent and incoherent de-dispersion.

pulse profile shape depends on the radio flux across the beam, which is not uniform and has a complicated structure (for further discussion, see Manchester et al., 2010). Therefore, the observed pulse profile may consist of several components in the main pulse or interpulse (e.g. Rankin, 1983a; Gangadhara \& Gupta, 2001). Figure 1.3 shows some examples of different integrated pulse profile shapes.

\subsubsection{Mass, radius, and moment of inertia of NSs}

The mass of a NS cannot be easily predicted theoretically due to the lack of knowledge of the equation of state. With different assumptions of equation of states 


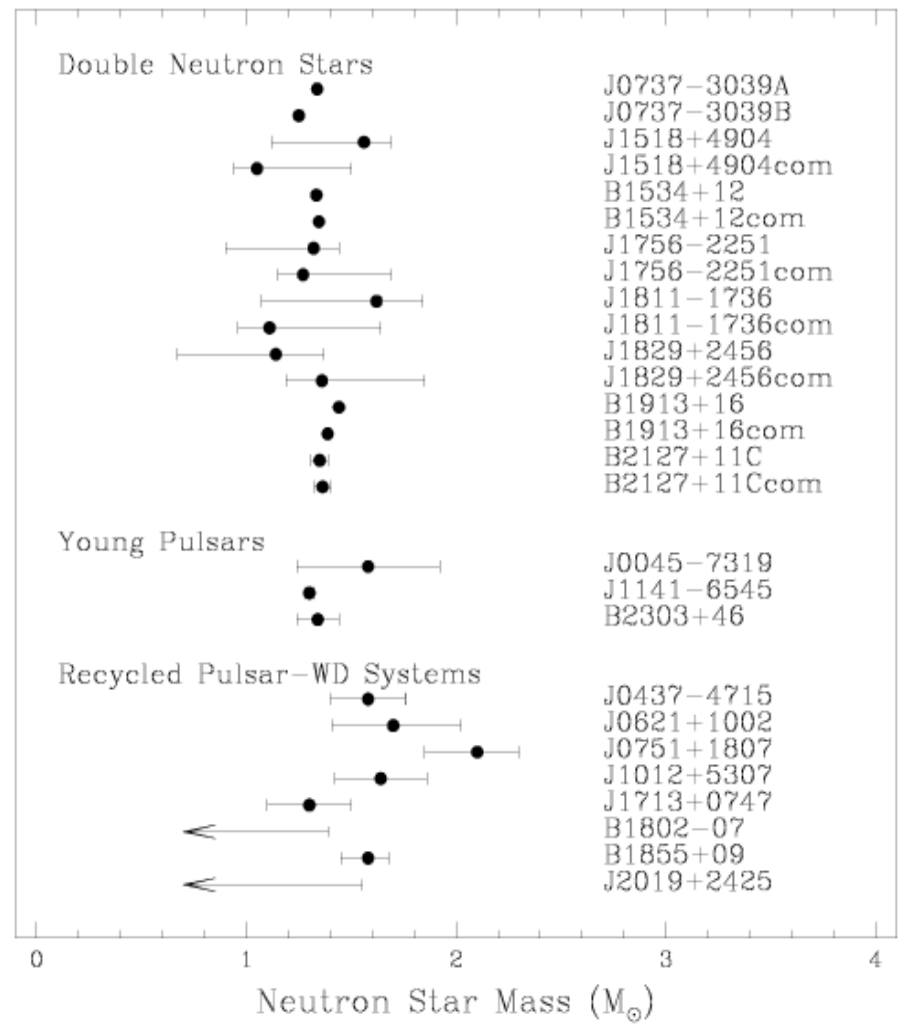

Figure 1.4: NS masses inferred from timing observations of binary pulsars (Lorimer \& Kramer, 2005). Note that the canonical NS mass is $1.4 \mathrm{M}_{\odot}$. The binary pulsars $\mathrm{J} 1906+0746$ and J1753-2240 are not included in this figure.

as given in Lattimer \& Prakash (2007), the maximum predicted NS mass is about $3 \mathrm{M}_{\odot}$. In observation, we are able to measure the NS mass when it is in a binary systems through pulsar timing using Keplerian and Post-Keplerian parameters. This is discussed in Section 1.7.1 in detail. Figure 1.4 summarizes the inferred masses of NSs in binary systems. The canonical mass of a NS is assumed be to $1.4 \mathrm{M}_{\odot}$, which is the Chandrasekhar mass. Recently, Demorest et al. (2010) measured the mass of the millisecond pulsar (MSP) J1614-2230 is to be $(1.97 \pm 0.04) \mathrm{M}_{\odot}$ from pulsar timing through the Shapiro delay signature. This is the largest measured mass for a NS so far.

The NS radius $\left(R_{N S}\right)$ is another property which we cannot obtain easily (see 
Lorimer \& Kramer, 2005). With a typical NS mass of $1.4 \mathrm{M}_{\odot}$, Lattimer \& Prakash (2007) showed that the radius of a NS is between 10-15 km. Generally, in most studies and in this work, the NS radius is taken to be $10 \mathrm{~km}$; i.e., $\mathrm{R}_{\mathrm{NS}}=10 \mathrm{~km}$.

The moment of inertia $(I)$ of NSs is obtained theoretically. For a sphere of uniform density, the moment of inertia can be written as $I=k M R^{2}$, where $k=2 / 5$, but the mass density of a pulsar is not uniform. Most models predict $k \sim 0.30-0.45$, so that in practical calculations, with $M=1.4 \mathrm{M}_{\odot}$ and $R=10 \mathrm{~km}$, the moment of inertia is $I=10^{45} \mathrm{~g} \mathrm{~cm}^{2}$.

\subsubsection{Rotational kinetic energy}

Pulsars are powered by loss of their rotational kinetic energy. Therefore, the spin period of a pulsar increases with time. With the rotational energy $E_{\text {rot }}=I \Omega^{2} / 2$, we write the rotational kinetic energy loss as

$$
\dot{E}=\frac{d E_{\mathrm{rot}}}{d t}=\frac{d\left(I \Omega^{2} / 2\right)}{d t}=I \Omega \dot{\Omega}=4 \pi^{2} I \dot{P} P^{-3}
$$

where $\Omega$ is the rotational angular frequency, $P$ is the spin period, and $\dot{P}$ is the

derivative of the spin period. This quantity $\dot{E}$ is known as the "spin-down luminosity" of the pulsar. The young pulsars are observed as bright sources due to high energy loss with small spin periods and larger period derivatives (e.g. Crab pulsar). By assuming $I=10^{45} \mathrm{~g} \mathrm{~cm}^{2}$, we can rewrite the equation as

$$
\dot{E}=3.95 \times 10^{31}\left(\frac{\dot{P}}{10^{-15}}\right)\left(\frac{P}{s}\right)^{-3} \mathrm{erg} / \mathrm{s}
$$


implying that for a pulsar with $P=1 \mathrm{~s}$ and $\dot{P}=10^{-15} \mathrm{~s} / \mathrm{s}$, the spin-down luminosity is $3.95 \times 10^{31} \mathrm{erg} / \mathrm{s}$.

The rotational kinetic energy of pulsars is dissipated through mechanisms such as nonthermal radiation (from radio to gamma-ray) and a relativistic wind (see Section 1.3). Therefore, the pulsar luminosity $L$ for a given frequency window must be less than the spin-down luminosity (i.e. $L<\dot{E}$ ). Note that we constrain the luminosity for gamma-ray emitting pulsars in Chapter 5.

\subsubsection{Magnetic field strength}

It is believed that pulsars have strong dipole magnetic fields. However, a direct measurement for the magnetic field of a pulsar is difficult to obtain. An indirect measurement can be made from cyclotron absorption lines in the spectrum of Xray binaries. We derive the magnetic field theoretically by using simple physics. From classical electrodynamics, we can write an equation for energy radiation by a rotating magnetic dipole with a magnetic moment $\vec{m}$ as (Jackson, 1962),

$$
\dot{E}_{\text {dipole }}=\frac{2}{3 c^{3}} m^{2} \Omega^{4} \sin ^{2} \alpha
$$

where $\alpha$ is the misalignment of the magnetic axis from the rotation axis and $c$ is the

speed of light. By equating the spin-down luminosity (Equation 1.1) with $\dot{E}_{\text {dipole }}$, we derive an expression for the rotational frequency evolution

$$
\dot{\Omega}=-\left(\frac{2 m^{2} \sin ^{2} \alpha}{3 I c^{3}}\right) \Omega^{3} .
$$


The magnetic moment is approximately equal to the magnetic field strength in the form of $B \approx m / r^{3}$, so that we get the surface magnetic field strength

$$
B_{\text {surf }}=B(r=R)=\sqrt{\frac{3 c^{3} I}{8 \pi^{2} R^{6} \sin ^{2} \alpha} P \dot{P}}
$$

For a typical NS with $I=10^{45} \mathrm{~g} \mathrm{~cm}^{2}$ and radius of $R_{\mathrm{NS}}=10 \mathrm{~km}$, we find

$$
B_{\text {surf }}=3.2 \times 10^{19} \sqrt{P \dot{P}} \text { Gauss } \simeq 10^{12}\left(\frac{\dot{P}}{10^{-15}}\right)^{1 / 2}\left(\frac{P}{s}\right)^{1 / 2} \text { Gauss }
$$

for an orthogonal rotator, $\alpha=90^{\circ}$. With measured $P$ and $\dot{P}$, the inferred surface magnetic fields of observed pulsars are $\sim 10^{7}-10^{14} \mathrm{G}$.

\subsubsection{Age estimate}

The rotational frequency evolution (Equation 1.4), more generally, can be written as a power law, in terms of pulse frequency $\nu$,

$$
\dot{\nu}=-K \nu^{n}
$$

where $n$ is known as the braking index and $K$ is a constant. For a pure magnetic dipole, the braking index is $n=3$ (see Equation 1.4 with the form $\dot{\Omega} \propto \Omega^{n}$ ). By using the observed $\ddot{\nu}$ for some pulsars along with their measured $\dot{\nu}$ and $\nu$, braking indices have been measured from $n=\nu \ddot{\nu} / \dot{\nu}^{2}$. The values range from $n=0.9$ to $n=2.9$ (Espinoza et al., 2011; Magalhaes et al., 2012), implying that the actual 
value is less than the pure dipole braking index of $n=3$. However, for simplicity, $n=3$ is assumed in most pulsar studies. Note that we place limits on the braking index for high-energy gamma-ray pulsars in Chapter 5.

The evolution of the pulse period $(P=1 / \nu)$ of the pulsar becomes $\dot{P}=$ $K P^{2-n}$. By integrating this first-order differential equation, we can derive an expression for the age of the pulsar

$$
T=\frac{P}{(n-1) \dot{P}}\left[1-\left(\frac{P_{0}}{P}\right)^{n-1}\right]
$$

where $P_{0}$ is the spin period at the birth. However, the initial spin period $P_{0}$ of the pulsar is difficult to obtain unless we know the true age independently and an accurate $n$ of the pulsar (e.g., for Crab, $P_{0} \approx 19 \mathrm{~ms}$ ). Therefore, the distribution of $P_{0}$ is not clearly understood. Recent studies estimate a wide range of initial spin periods $P_{0} \in[15 \mathrm{~ms}, 150 \mathrm{~ms}]$ (see, Migliazzo et al., 2002; Kramer et al., 2003), while Watters \& Romani (2011) used a short initial spin period $P_{0} \approx 50 \mathrm{~ms}$. Note that we place limits on $P_{0}$ for gamma-ray pulsars in Chapter 5. Assuming that the initial spin period is much shorter than the present value $\left(P_{0} \ll P\right)$ and the spin-down is due to magnetic dipole radiation $(n=3)$, Equation (1.8) simplifies to the characteristic age of

$$
\tau_{c}=\frac{P}{2 \dot{P}} \simeq 15.8\left(\frac{P}{s}\right)\left(\frac{\dot{P}}{10^{-15}}\right)^{-1} \mathrm{Myr} .
$$

This equation gives an approximate value for the age of a pulsar based on its period and period derivative. For an instance, the characteristic age of the Crab pulsar 
is $1240 \mathrm{yr}$, but the true known age is about $950 \mathrm{yr}$. Therefore, this is a crude estimation.

Sometimes, pulsar ages are derived from their measured transverse velocities (from pulsar timing) and distances from the Galactic plane. Assuming pulsars were born in the Galactic plane, this "kinetic age" is computed by extrapolating their velocities back to the plane. In general, the kinetic age is roughly consistent with the characteristic age for young pulsars. However, they significantly deviate from each other for older pulsars due to their large characteristic ages. A possible explanation is that the period derivative $\dot{P}$ is smaller for old pulsars and hence, the characteristic age is much larger than the kinetic age (Lyne \& Smith, 2004).

\subsection{5 $P-\dot{P}$ diagram of pulsars}

As discussed above, pulsar properties such as spin-down luminosity, magnetic field strength, and characteristic age are obtained from the pulsar timing inferred period and period derivative (see Section 1.6 for a detailed discussion about the pulsar timing). Therefore, we can present these properties and the location of the pulsar in period versus period-derivative space. This plot is known as $P-\dot{P}$ diagram and is shown in Figure 1.5 (Note that the two axes are in logarithmic scale).

According to the $P-\dot{P}$ diagram, we can classify radio pulsars mainly into two categories, namely canonical pulsars (CPs) which have periods of $\sim 1 \mathrm{~s}$ and fast spinning millisecond pulsars (MSPs). Most of the MSPs are in binary systems with a companion star. This population is very old with characteristic ages of $>100 \mathrm{Myr}$ 


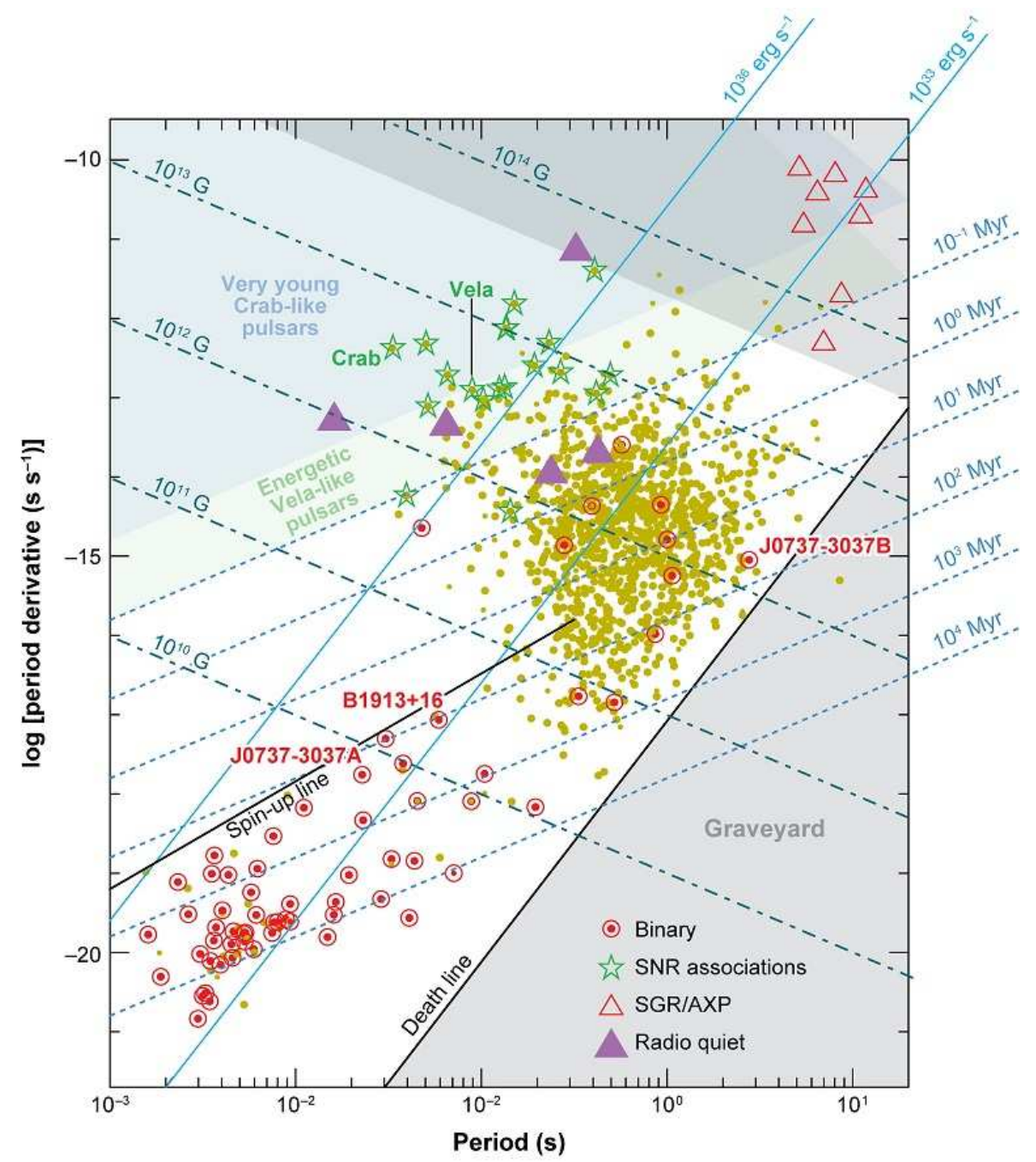

Figure 1.5: $P-\dot{P}$ diagram for known pulsars (Kramer \& Stairs, 2008). The spindown luminosity, magnetic field and the characteristic age are marked with different lines. Dots and circle-dots represent isolated pulsars and binary pulsars, respectively. Open stars represent pulsars with supernova remnant (SNR) associations and open triangles represent soft gamma-ray repeaters (SGR) and anomalous X-ray pulsars (AXP). Filled triangles represent radio quiet pulsars. 
and are comprised of stable rotators. We believe that MSPs were CPs and then recycled from their binary companion. Thus, during the recycling process, the old $\mathrm{CP}$ accretes materials from the companion star. This process transfers the angular momentum to the pulsar and spins it up to millisecond periods. The fastest spinning MSP is PSR J1748-2446ad (a binary MSP located in the globular cluster Terzan 5), which has a spin period of $1.396 \mathrm{~ms}$ (Hessels et al., 2006).

\subsubsection{Pulsar distances}

In general, pulsar distances are determined from three different methods, namely parallax measurements, HI measurements, and DM-derived distances. The pulsar distances used in Chapter 5 are mainly estimated from these three methods.

\subsubsection{Parallax measurements}

The pulsar parallax generally measures the curvature of the wavefront arriving from the pulsar. This can be done by measuring the apparent position change of the pulsar with the Earth orbital motion around the Sun, or by precise measurements of pulse times of arrival. In order to study the apparent position, we need to measure the pulsar position precisely, which can be obtained by long-baseline interferometers. This method provides the best model-independent pulsar distances. The other method is done through high-precision pulsar timing. In both methods, we need to observe the pulsar for at least a complete year. By measuring the parallax precisely, the distance to the pulsar is obtained as $1 / p$, where $p$ is the parallax angle in arc- 
seconds. However, this technique is applicable to nearby pulsars in general, because the parallax angle becomes very small for distant pulsars $(\geq 2 \mathrm{kpc})$ and difficult to measure.

\subsubsection{HI measurements}

In this technique, we measure the neutral hydrogen (HI) emission and absorption features in pulsar data. If there is a HI distribution in front of the pulsar along the line of sight, we are likely to detect absorption by this region. Similarly, if the HI region is behind the pulsar, an emission feature can be detected in the pulsar observation. Therefore, by modeling the Doppler shifts of emission and absorption features with a Galactic rotation curve along the direction of the pulsar, we are able to constrain the upper and lower limits of the distance to the pulsars.

\subsubsection{DM-derived distances}

This method is heavily used in radio pulsar astronomy. The pulsar signal propagates through the cold ionized plasma in space (interstellar medium - ISM) along the line of sight before it reaches the Earth. The refractive index of the ISM is frequency dependent in a way such that the high frequency signal from the pulsar reaches the Earth earlier than the low frequency signal. Therefore, within the observing bandwidth, we need to correct for this delay and align all the pulses in order to form a proper pulse profile. Otherwise the dispersed pulses lead to a much

broader pulse profile compared to the intrinsic profile. Then the time of arrival of 
the pulse obtained from this smeared profile is less precisely measured than that of the intrinsic pulse, contributing to timing error.

The time delay between two frequencies $f_{1}$ and $f_{2}$ can be given as

$$
\Delta t \simeq 4.15 \times 10^{6} \mathrm{~ms} \times\left(f_{1}^{-2}-f_{2}^{-2}\right) \times D M
$$

where the dispersion measure (DM) can be written as

$$
D M=\int_{0}^{d} n_{e} d l \mathrm{~cm}^{-3} \mathrm{pc} .
$$

Simply, the DM gives the electron density $n_{\mathrm{e}}$ along the line of sight between the pulsar and the Earth (for the pulsar distance $d$ ). Therefore, by correcting for time delays between pulses across the frequency band, we can make a de-dispersed pulse profile. This time correction is done through pulsar timing by fitting for the best DM. Finally, the inferred DM can be used to determine the distance to the pulsar for a given $n_{e}$ model (Equation 1.11). By using the Galactic spiral arm structure, Cordes \& Lazio (2002) developed an electron density model (NE2001) which can be used to obtain $n_{e}$ for a given line of sight. In order to calibrate this model, they used independent pulsar distance estimates obtained from parallaxes and HI measurements. 


\subsection{The pulsar magnetosphere}

Pulsar magnetospheres are not completely understood due to their complicated structure. There are a few models put forward to explain the structure, but none of these models are a realistic form of the pulsar magnetosphere. However, the current understanding of the NS magnetosphere and its characteristics are capable of explaining some main features of observed electromagnetic radiation such as pulse profile shapes, linear polarization characteristics, and pulsar geometry, in general. This subsection mainly discusses the theoretical background of pulsar magnetospheres and then extends it to pulsar emission mechanisms.

With the discussion of Deutsch (1955), a NS can be idealized as a completely conducting, sharply bounded sphere, rigidly rotating in a vacuum. Based on their investigation, Goldreich \& Julian (1969) proposed a simplified, but useful, model to explain pulsar electrodynamics. In their model, the pulsar is an aligned rotator (i.e. the magnetic axis is aligned with the rotation axis) with a spin angular velocity $\Omega$. They assumed that the NS has a dipolar magnetic field and both the interior and exterior of the star are excellent electrical conductors. Therefore, with rotation, the star will be polarized and the surface charge density is quadrupolar, so that the outside electric field is a quadrupole (see Michel, 1991). The electric field component which is parallel to the magnetic field line on the NS surface is

$$
E_{\|}(r=R)=\left.\frac{\vec{E} \cdot \vec{B}}{B}\right|_{r=R}=-\frac{\Omega B_{0} R}{c} \cos ^{3} \theta
$$

where $B_{0}$ is the polar magnetic field, $R$ is the NS radius, $c$ is the speed of light, 
and $\theta$ is the polar angle. The outward electric force $\left(F=q E_{\|}\right)$exerted on the charged particles on the NS surface is much greater than the inward gravitational force on them. Therefore, these charged particles leave the NS surface and enter the magnetosphere. Thus, the assumed vacuum condition outside the NS no longer exists, but rather the magnetosphere is filled with a dense plasma with a local charge distribution of

$$
\rho_{e}(r, \theta)=\frac{\vec{\nabla} \cdot \vec{E}}{4 \pi}=-\frac{\vec{\Omega} \cdot \vec{B}}{2 \pi c}=-\frac{B_{0} \Omega R^{3}}{4 \pi c r^{3}}\left(3 \cos ^{3} \theta-1\right) .
$$

Once this charge distribution is arranged in the magnetosphere, the parallel component of the electric field to the magnetic field becomes zero $(\vec{E} \cdot \vec{B} \sim 0)$. In other words, the parallel electric field component is screened from these particles and a force-free condition is maintained outside the star. With the above charge distribution, it is clearly seen that the charges above the equatorial region and the poles are opposite in sign, whereas $\rho_{e}$ changes sign when the polar angle $\theta$ is at $\cos \theta=1 / \sqrt{3}$. This surface is denoted as the "null-charge surface", where $\vec{\Omega} \cdot \vec{B}=0$. Figure 1.6 shows the particle distribution in the magnetosphere according to Goldreich \& Julian (1969). The particle number density at the magnetic pole on the NS surface $n_{G J}=7 \times 10^{10} P^{-0.5} \dot{P}^{0.5}$, where $P$ in seconds and $\dot{P}$ in units of $10^{-15}$, is known as the "Goldreich-Julian (GJ) density".

The drift velocity $V_{D}=(\vec{E} \times \vec{B}) / B^{2}$ on charged particles is in the azimuthal direction $\hat{\phi}$, which forces them to corotate with the NS. This corotation is limited up to the light cylinder radius $\mathrm{R}_{\mathrm{LC}}=c P / 2 \pi$, where the corotational velocity reaches 


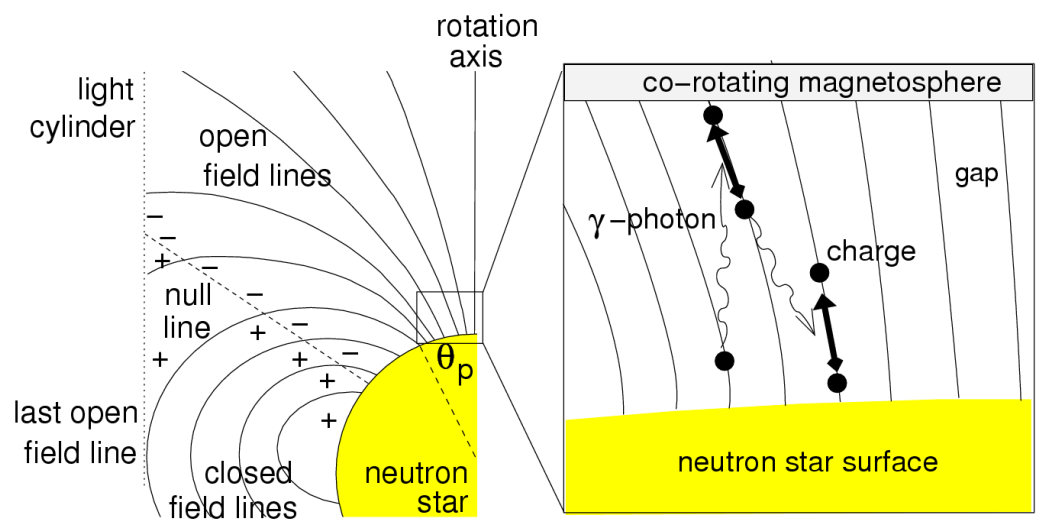

Figure 1.6: Pulsar magnetosphere in the Goldreich-Julian model. The pair creation in the polar gap is indicated (Lorimer \& Kramer, 2005).

the speed of light. This is the boundary of the magnetosphere. The magnetic field lines which are closed within the light cylinder are referred to as closed field lines and the field lines that do not close are known as open field lines. The polar cap (PC) region is defined by the footpoints of the last closed field lines (i.e. the field lines which just touch the light cylinder) on the NS surface. Therefore, the footpoints of all the open field lines are located inside the PC region.

With this model, the particles corotate with closed field lines in the magnetosphere. However, above the PC, the corotating particles can flow out from the light cylinder along the open field lines due to the centrifugal force. Therefore, the particle density drops from the GJ density and the previously screened $E_{\|}$component is no longer zero $(\vec{E} \cdot \vec{B} \neq 0)$. This breaks the force-free condition in the magnetosphere. Thus, the charged particles accelerate with $E_{\|}$component along open magnetic field lines and produce electromagnetic radiation.

In general, there are few regions located in the pulsar magnetosphere in which 
the GJ density is violated. In these regions, particle depletion occurs and $E_{\|}$cannot be screened. These regions are called acceleration gaps. The electromagnetic radiation is mainly generated with these gaps. The commonly identified gaps are the polar gap (or the inner magnetosphere gap) and the outer magnetosphere gap (see Figure 1.2). The different emission properties of these gaps are discussed in the next few sections.

\subsubsection{Pulsar radio emission}

Observations show that radio pulsars have smaller duty cycles (i.e., narrower pulse components) when compared to high-energy emission. Therefore, the radio emitting region is likely to be localized to a narrower region in the magnetosphere. Although the the radio emission mechanism is not completely understood, three types of techniques are widely used in explaining the pulsar radio emission. In order to obtain the observed high brightness temperature of pulsar radio emission, which is about $10^{25}-10^{30} \mathrm{~K}$, the radiation has to be generated coherently. The three techniques which can produce coherent emission are curvature radiation $(\mathrm{CR})$ involving an antenna mechanism, relativistic plasma emission, and maser emission. In all these techniques, the particles stream along magnetic field lines and emit radiation tangent to the field line at the emission point. As an introduction to our work presented in later chapters, we primarily discuss the antenna mechanism based on pulsar PC geometry as the radio emission mechanism (Ruderman \& Sutherland, 1975). We briefly describe the other two coherent emission techniques later in this 
section.

According to the antenna mechanism, the charged particles are bunched to generate coherent radiation. The basic idea is that if $N$ particles of charge $q$ are confined in a volume of size less than half of the emitted wavelength, then these will radiate in phase like a particle of charge $N q$. Then the emitted power is $N^{2}$ times the power radiated by one single particle. The most common radio emission mechanism was proposed by Ruderman \& Sutherland (1975) based on the antenna mechanism involving a $\mathrm{PC}$ model in which the radio emission is generated from the accelerated particles within the polar gap. The polar gap is a charge depleted region located within the PC bounded by the last closed field lines and extended upwards from the NS surface. When the charged particles leave the magnetosphere through the light cylinder the polar gap is formed. Within the gap, the force-free condition is violated $(\vec{E} \cdot \vec{B} \neq 0)$, but the rest of the magnetosphere is force-free. The parallel component of the electric field at the pole within the gap is

$$
E_{\|}=2 \frac{\Omega B_{s}}{c}(h-z)
$$

where $B_{s}$ is the surface magnetic field, $h$ is the polar gap height, and $z$ is the distance from the NS surface. Therefore, the potential drop across the gap is written as

$$
\Delta V=\frac{\Omega B_{s} h^{2}}{c}
$$

These charged particles are known as "primary particles". They accelerate through non-zero electric field component in the gap and gain extremely relativistic energies. 
For a pulsar with $B_{s}=10^{12} \mathrm{G}, \Omega=2 \pi \mathrm{s}^{-1}$, and $h=10^{3} \mathrm{~cm}$, the energy becomes $e \Delta V \geq 10^{11} \mathrm{eV}$. When these particles accelerate along magnetic field lines, CR is emitted with photon energies of

$$
E_{p h}=\hbar \omega=\frac{3 \gamma^{3} \hbar c}{2 r_{c}}
$$

where $\gamma$ is the Lorentz factor and $r_{c}$ is the field line radius of curvature. When a CR photon exceeds the energy $2 m c^{2}$, a $e^{+}-e^{-}$pair is formed (known as pair cascade), where $m$ is the particle mass (see Figure 1.7). This pair can discharge the gap and significantly change $E_{\|}$. Due to the discharge, a pair formation front occurs at a height $h$ and is the upper boundary of the gap. The newly generated pairs are so called "secondary particles" and have low energies compared to those of primary particles. These secondary particles then move to the force-free region in the magnetosphere above the polar gap and travel with a constant velocity along the magnetic field line.

The characteristic frequency of the CR photon is deduced from Equation 1.16 as $\omega_{c}=3 \gamma^{3} c / 2 r_{c}$. A primary particle can gain the maximum speed, $\gamma=1.2 \times 10^{7}$, when it crosses the entire gap height. Then, for $r_{c} \approx 10^{6} \mathrm{~cm}$, the CR photon energy is $E_{p h} \approx 800 \mathrm{MeV}$ and therefore the frequency is $\omega_{c} / 2 \pi \approx 10^{23} \mathrm{~Hz}$. This radiation is in the gamma-ray regime. However, the secondary particles have much lower energies $(\gamma \approx 800)$ and for $r_{c} \approx 10^{9} \mathrm{~cm}$, the CR frequency becomes $\omega_{c} / 2 \pi=10^{9} \mathrm{~Hz}$, which is in the radio band. Therefore, the secondary particles produce radio emission in pulsars. 


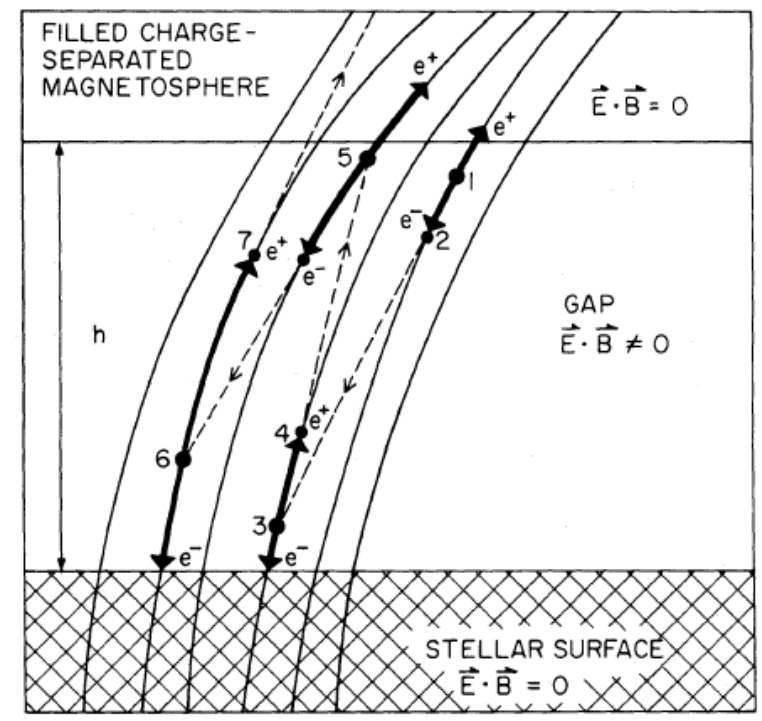

Figure 1.7: Pair cascade in the polar gap of the pulsar magnetosphere (Ruderman \& Sutherland, 1975). A primary particle produced photon discharges to an $e^{+}-e^{-}$ pair at 1 . Then the secondary $e^{+}$enters to the force-free magnetosphere above the gap and $e^{-}$accelerates toward the surface and radiates a $\mathrm{CR}$ photon at 2. This photon again discharge to a pair at 3. This process continues until the CR photons do not have enough energy to produce pairs, where energy $<2 m c^{2}$.

The next argument in Ruderman \& Sutherland (1975) is that if the radio emission is generated incoherently, then the brightness temperature of the emission should be lower than what we measure from pulsars. Therefore, the radio emission should be generated coherently. Both primary and secondary particles are involved in this coherent mechanism. The secondary particles have lower kinetic energy and therefore move with a slower speed compared to the primary high energy particles. Thus, a beam of primary particles catches up secondary particles within the forcefree magnetosphere. This beam leads to the bunching of secondary particles and enhances the CR, resulting in radio emission of high brightness temperature.

Although the coherent CR emission by bunches explains the pulsar radio emission to some extent, there are serious drawbacks to this technique, mainly including 
the lack of a satisfactory mechanism to produce bunches and the inability to maintain these bunches over a characteristic time of emission (Melrose, 1992). As a solution, two better techniques have been proposed: (a) relativistic plasma emission and (b) maser emission. In relativistic plasma emission (Melrose, 1995), the radiation in the magnetosphere is assumed to be generated from relativistically streaming 1D pair plasma. When a particle is moving relativistically through the pair plasma, different waves can be generated due to a streaming instability. Then a portion of the energy of these waves converts to coherent radiation through nonlinear processes. However, the growth rate of these instabilities are too small, so that the pair plasma leaves the magnetosphere before it results in significant energy. Also, large growth rates can produce a sequence of beams. The third technique, maser curvature emission, was proposed with either curvature-drift (Luo \& Melrose, 1992) or field-line torsion (Luo \& Melrose, 1995). In this mechanism, the absorption coefficient should be negative in order to have particle growth, or wave amplification. According to the curvature-drift maser model, this condition is satisfied only when the particle drift velocity is non-zero and the Lorentz factor is above a relatively high threshold $\gamma \geq 10^{4}$ with magnetic fields of $B>10^{8} \mathrm{G}$. The torsion-driven maser requires that the field line is not to be confined to a plane, so that it has two different radii of curvature in orthogonal directions to have a negative absorption. In this mechanism, the growth is possible above $\gamma \geq 40$, so that it is compatible with pair cascade models. 


\subsubsection{Pulsar gamma-ray emission}

The mechanisms of high-energy gamma-ray emission of pulsars involve both primary and secondary particles. The understanding of the mechanism is a step ahead compared to the radio emission, because coherence is not necessary. However, the emission locations in the magnetospheres are still not clearly understood. Since the observed gamma-ray pulse profiles have much larger duty cycles compared to the radio emission, it is believed that the gamma-ray emission has a wider emission beam. Daugherty \& Harding (1982) simulated the electromagnetic cascade within the polar gap region and showed the gamma-ray emission is produced through primaries and secondaries. However, wider pulse profiles and bridge emission in gamma-ray pulsars are ideally explained with emission generation in outer magnetosphere regions. Furthermore, the shift in spin longitude of gamma-ray and radio pulse profile peaks of most of the radio and gamma-ray loud pulsars reveals that the two different energy emissions are generated in different locations in the magnetosphere.

As proposed in Cheng et al. (1986) and further investigated in Chiang \& Romani $(1992,1994)$ and fully constructed in Romani (1996), the "outer gap" (OG) emission model is widely used in explaining gamma-ray emission of pulsars. According to the OG model, the emission is generated in the outer magnetosphere gap which is located above the null-charge surface (i.e. the surface where $\vec{\Omega} \cdot \vec{B}=0$ ) towards the $\mathrm{R}_{\mathrm{LC}}$. The lower surface of the gap is the last closed field lines and the upper surface is defined with a fractional gap width (see Figure 1.8). 


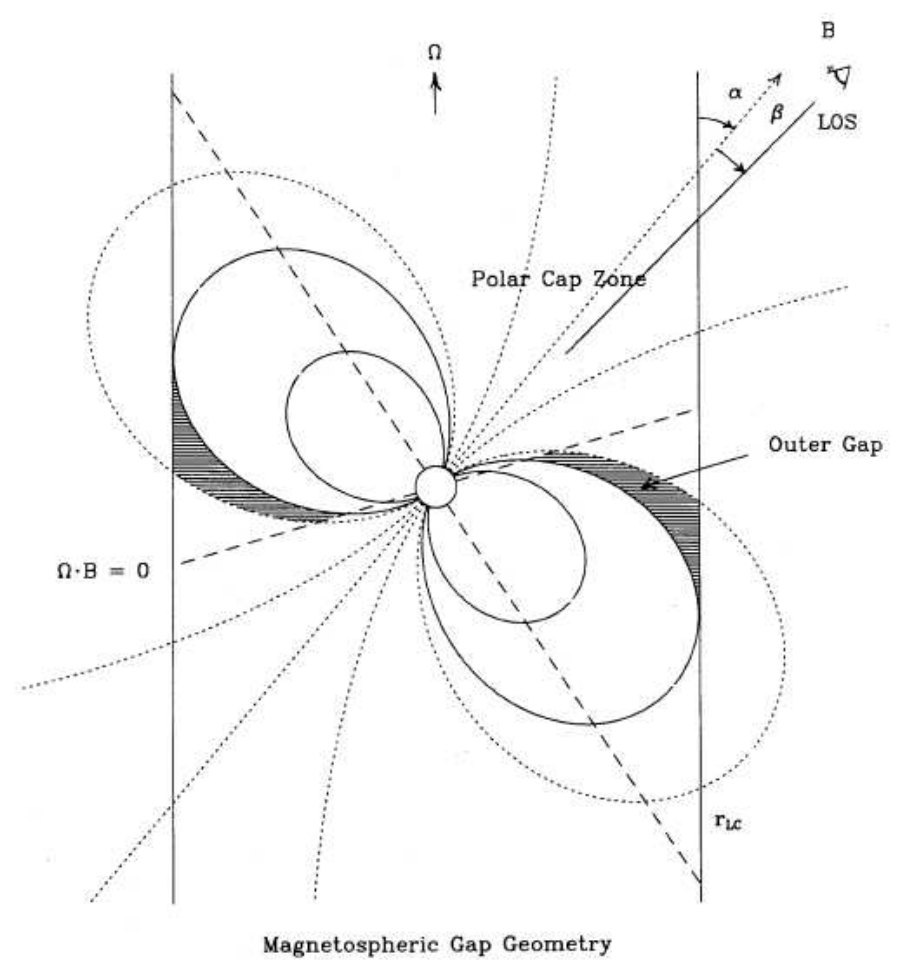

Figure 1.8: A schematic view of the OG emission model. The magnetic axis of the pulsar is misaligned by an angle $\alpha$ with respect to the rotation axis. The impact parameter $\beta$ is the closest approach of the magnetic axis to the line of sight. The gap (shaded region) is bound from the null-charge surface (dashed lines), the light cylinder, and the last closed field lines. The gap fractional thickness is $w$ (Chiang \& Romani, 1992). 
The primary particles are accelerated in this charge-depleted region and radiate primary curvature photons. These primary photons have moved away from the field line due to pulsar rotation or aberration and then the secondary pairs are produced with a considerable pitch angle. Consequently, with momentum perpendicular to the field line, the secondary pairs radiate synchrotron photons. Due to pair production, the charged particle density increases in the gap, so that the upper boundary depends on the pair cascade. The curvature photons contribute to the hard gamma-ray region and synchrotron photons contribute to the soft gamma-ray region of the spectrum.

Based on the same electromagnetic pair cascade mechanism, but in different charge-depleted gap locations in the magnetosphere, several other outer magnetosphere models propose to explain the gamma-ray emission of pulsars. Among these, the "two pole caustic" (TPC) model (Dyks \& Rudak, 2003) and the "slot gap" (SG) model (Muslimov \& Harding, 2003, 2004) are commonly used. In the TPC model, the emission region is within the gap region around the last closed field lines and extending from the NS surface to the light cylinder. In the SG model, the charge depleted tube-like region is bounded between the last closed field lines and the pair formation front (see Figure 1 in Muslimov \& Harding, 2003). In special cases such as the Crab pulsar and B1937+21, the peaks of radio and gamma-ray pulse profiles are in close alignment, implying that both the radio and high-energy emission are generated within the outer magnetosphere (Cheng \& Ruderman, 1977).

All of these pulsar emission geometry models use rotating dipole field line structure in a vacuum and assume that the magnetosphere is almost force-free ex- 
cept in gap regions. However, in the real force-free condition, the structure of the magnetosphere should be changed significantly from the rotating dipole field due to plasma current (i.e., $\rho \vec{E}+\vec{j} \times \vec{B} / c=0$, where $\vec{j}$ is the current density). Spitkovsky (2006) found a numerical solution for a time-dependent force-free pulsar magnetosphere. Since the force-free magnetosphere has an $E_{\|}=0$ everywhere, the particle acceleration, and hence the radiation, cannot be explained. Bai \& Spitkovsky (2010a) used the OG and the SG geometry along with the force-free magnetosphere field structure to produce gamma-ray pulse profiles. We also note that, with the SG geometry, Harding et al. (2011) found that the rotating dipole magnetosphere in vacuum provides better fits to the observed gamma-ray profiles than the force-free magnetosphere. With this information, a real pulsar magnetosphere should lie between vacuum and force-free conditions. Therefore, a magnetosphere with a finite conductivity is a better approach. According to Kalapotharakos et al. (2012b), the pulsar magnetosphere has a conductivity $\sigma$ that deviates in structure from vacuum or force-free condition (i.e., $\sigma=0$ gives the vacuum approximation and $\sigma \rightarrow \infty$ gives the force-free approximation). This model has an $E_{\|}$component which can accelerate particles and produce gamma-ray emission (Kalapotharakos et al., 2012a). This model is still in development. Perhaps, this will provide a more realistic pulsar magnetosphere in the future. 


\subsection{Pulsar geometry with polarization studies and the rotating vector model}

Pulsar radio emission is highly polarized. The four Stokes parameters, $I, Q$, $U$, and $V$, of the radio emission can be obtained by using a polarimeter, where $I$ is the total intensity, $L=\sqrt{Q^{2}+U^{2}}$ is the linearly polarized intensity, and $V$ is the circular polarized intensity. By studying polarization data of pulsars, Gould \& Lyne (1998) found that the average degree of linear polarization $\langle L / I\rangle$ is about $20 \%$ and circular polarization is about 10\%. However, some pulsars show $100 \%$ polarized radio emission, implying that the degree of polarization of pulsars has a wide range. Another information is the variation of the linear polarization position angle (PPA) (i.e., $\left.\psi=\frac{1}{2} \arctan (U / Q)\right)$ as a function of the pulse phase. As noted in Radhakrishnan \& Cooke (1969) for the first time, this angle varies smoothly across the pulse component, giving a characteristic S-shaped curve (see Figure 1.9).

Although the radio emission mechanism is not well established, the linear and circular polarization can be explained qualitatively, with the presence of two orthogonal polarization modes. Based on Gil \& Snakowski (1990a,b) and Gil et al. (1993), the two polarization modes are $\epsilon_{\perp}$ perpendicular to the plane containing the dipole field line and $\epsilon_{\|}$in the plane parallel to the rotation axis. Adopting these two modes for each particle, these studies explain circular and linear polarization by using a superposition of emission from individual particles. Further, the observationally evident sense-reversal of circular polarization (Cordes et al., 1978) can be explained using these two orthogonal modes. The observed S-shaped PPA swing 


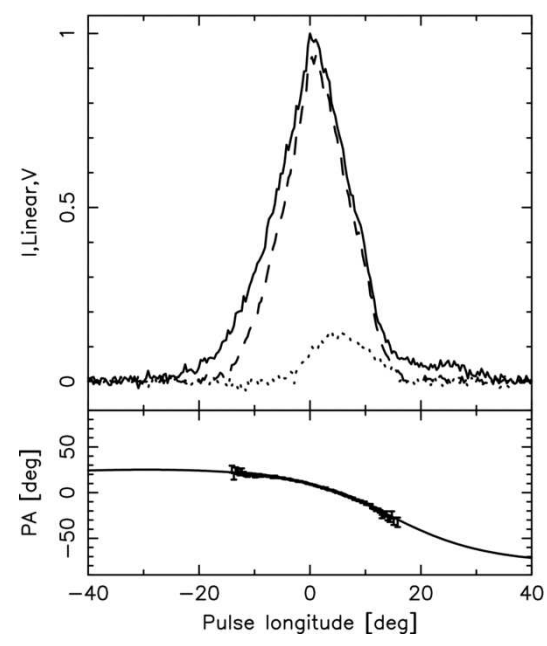

(a)

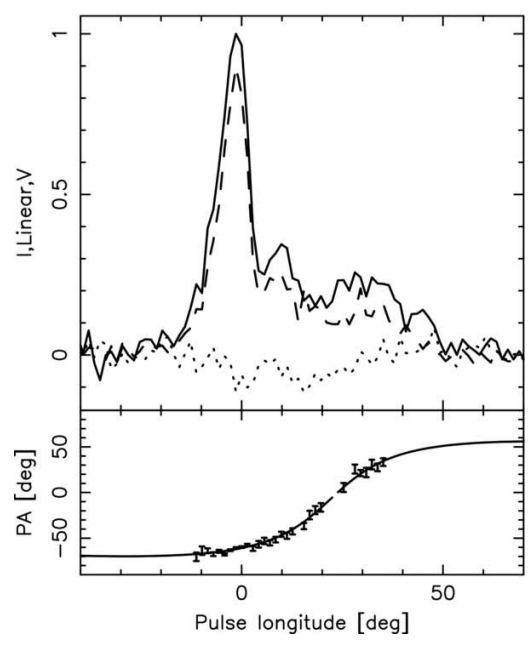

(b)

Figure 1.9: Polarization profiles for (a) PSR J0659+1414 (Arecibo data at 1418 $\mathrm{MHz}$ ) and (b) PSR J1718-3825 (Parkes data at $1369 \mathrm{MHz}$ ). The total intensity (solid), linear polarization (dashed), and circular polarization (dotted) of the onpulse region are shown. The bottom panel shows the variation of measured PPA angle across the pulse. The solid line is the RVM predicted PPA. These figures are taken from Weltevrede et al. (2010).

was explained by the presence of two different propagation effects for the two modes in the magnetosphere (i.e., there are two different refractive indices in orthogonal directions $n_{\|}$and $n_{\perp}$ ). The observed orthogonal jumps of the PPA swing was explained as switching the dominant orthogonal mode. In other words, the dominant polarization mode can suddenly switch from $\epsilon_{\perp}$ to $\epsilon_{\|}$, or vice-versa, resulting in a $90^{\circ}$ jump in PPA. According to Gil \& Snakowski (1990b), the PPA swing is not a geometrical issue, rather it is theoretical.

However, Radhakrishnan \& Cooke (1969) proposed a method to model the PPA swing including the geometry of the pulsar. This is called the "rotating vector model" (RVM). The model assumes that the emission is generated along the plane of the magnetic field line, assuming a pure dipole configuration, so that the polarization is in this plane. When the radio beam is sweeping the line of sight, the PPA varies 
because the projection of the field line in the sky plane varies. This geometrical PPA variation also has a S-shaped swing as we observe. According to the model, the PPA angle $\psi$ is written as

$$
\tan \left(\psi-\psi_{0}\right)=\frac{\sin \alpha \sin \left(\phi-\phi_{0}\right)}{\sin (\alpha+\beta) \cos \alpha-\cos (\alpha+\beta) \sin \alpha \cos \left(\phi-\phi_{0}\right)}
$$

where $\phi$ is the rotational phase, $\alpha$ is the magnetic misalignment, and $\beta$ is the impact parameter (or the closest approach of the magnetic moment with respect to the line of sight). The longitude of the fiducial plane is $\phi_{0}$ and the PPA at this longitude is $\psi_{0}$. By fitting the observed PPA swing to the model-estimated swing for a given $\phi$ based on the above equation, we can constrain the geometry angles $\alpha$ and $\beta$ of the pulsar. In general, these angles are associated with large errors due to two reasons; (a) the width of the radio pulse profile is small, so that there are few bins to sample the PPA, (b) PPA shows orthogonal jumps across the spin phase for some pulsars. This model is very useful in determining the geometry of pulsars, so it is widely used in pulsar astronomy. Rankin $(1983 \mathrm{a}, \mathrm{b}, 1990,1993 \mathrm{~b})$ reported radio polarization measurements of about 150 pulsars and determined their geometrical angles $\alpha$ and $\beta$ from the RVM. Mitra \& Rankin (2011) further analyzed 50 pulsars which have asymmetric pulse profiles and polarization position angles and determined their geometry. Rankin (1990) found that the range of $\alpha$ values is broad and has a peak around $35^{\circ}$.

Furthermore, the polarization data of some pulsars show that the steepest 
gradient of the PPA (i.e., $\sin \alpha / \sin \beta$ ) is not aligned with the pulse peak and shifted in spin longitude $\phi$. By assuming that the emission is generated at a height above the $\mathrm{PC}$ region, this shift $\delta \phi$ can be explained through aberration and retardation effects (Blaskiewicz et al., 1991). With this idea, we can estimate the emission altitude from $h_{e m} \approx \delta \phi c P / 4 \pi$, where $P$ is the spin period and $c$ is the velocity of light (e.g., Weltevrede et al., 2010).

\subsection{Pulsar radio beams and emission altitudes}

As shown in Figure 1.3, pulse profiles of pulsars have different shapes, varying from single peak to several components. By analyzing various pulse profile shapes with their geometries, Rankin (1983a,b, 1990, 1993a) proposed that the pulsar radio beam can be understood as a core and a set of conal components. Then the observed pulse profile shape depends on the beam structure and the relative motion between the line of sight and the radio beam. This results in different profile shapes according to which section of the beam structure that our line of sight encounters with the pulsar rotation (see Figure 1.10). However, some complex profile shapes cannot be explained with this simple core and conal beam structure. Therefore, Lyne \& Manchester (1988) proposed that pulsar beam may have a random patchy structure, so that the flux is larger in some patches. These two beam models are widely used in explaining pulse profiles and time-evolving pulse shapes of pulsars. We use Rankin's core and conal emission component beam model to explain pulse profile characteristics of PSR J0737-3039A/B in later chapters. 


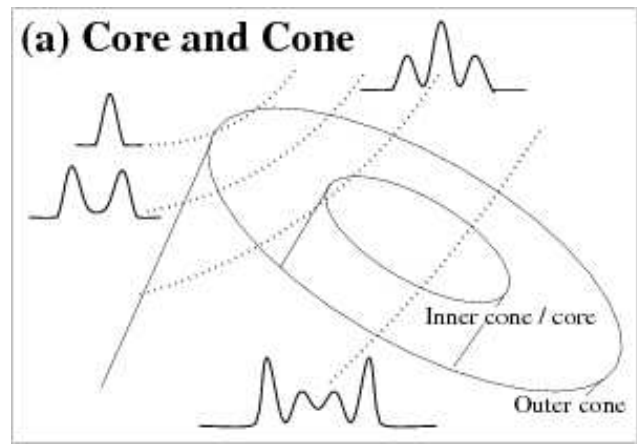

\section{(b) Patchy Beams}

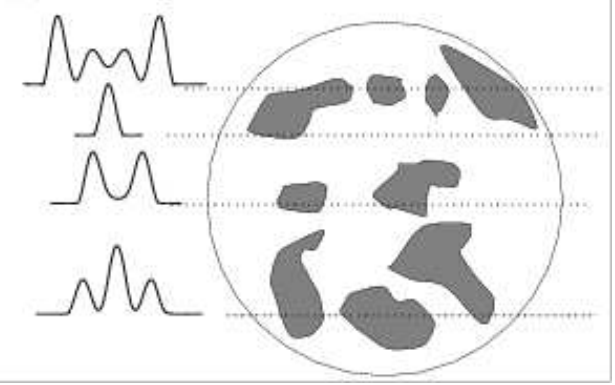

Figure 1.10: Schematic views of different beam structures (Lorimer \& Kramer, 2005). Dotted lines are different lines of sight across the beam and resultant pulse profiles are shown for (a) core and conal beam structure (b) patchy beam structure.

Although the pulsar radio emission mechanism is not well established, we can explain some useful basic properties with CR as discussed in Section 1.3.1. According to the NS dipole field line structure, the size of the PC region is dependent on the period of the pulsar. Therefore, the half-opening angle $\rho$ of the radio beam is dependent on the pulsar period $P$. For a small polar emission angle $\theta_{e m}$, the halfopening angle is $\rho \approx 3 \theta_{\mathrm{em}} / 2=\sqrt{9 \pi h_{\mathrm{em}} / 2 c P}$, where $h_{e m}$ is the emission altitude. With empirical fits, Rankin (1993a) showed that the half-opening angle of the radio conal beam is $\rho=5.8^{\circ} P^{-0.5}$, where the period $P$ is in seconds. This is a good approximation for canonical pulsars, but it is uncertain for MSPs.

As shown in Ruderman \& Sutherland (1975), radio emission generation is lowaltitude and centered around the magnetic axis. With $\mathrm{CR}$, the emission region in the magnetosphere is frequency dependent. As shown in Section 1.3.1, the characteristic frequency of $\mathrm{CR}$ is $\omega_{\mathrm{c}} \propto \gamma^{3} c / r_{\mathrm{c}}$, where $\gamma$ is the Lorentz factor and $r_{\mathrm{c}}$ is the magnetic field radius of curvature. With the dipole magnetic field, $r_{\mathrm{c}}$ increases with altitude. Therefore, according to the PC model, the high-frequency component is generated close to the NS surface and the resultant pulse profile is narrow, 
because the active region in the $\mathrm{PC}$ at low-altitude is narrower. The low-frequency component is generated at a higher altitude and then the profile is much wider. This is observationally evident. In normal isolated pulsars, radio emission heights have been estimated from their emission geometry inferred from radio polarization combined with the RVM (Section 1.4) and the pulse profile widths (Gil \& Kijak, 1993; Kijak \& Gil, 1997; Kijak \& Gil, 2003). By using data for several pulsars, Kijal \& Gil (1998) and (Kijak \& Gil, 2003) derived the pulsar emission altitude as

$$
r_{\mathrm{em}}=(400 \pm 80) f^{-0.26} \dot{P}^{0.07} P^{0.30} \mathrm{~km}
$$

where the frequency $f$ is in $\mathrm{GHz}, \dot{P}$ is in $10^{-15}$, and $P$ is in seconds. In general, the radio emission originates at $<100$ NS radii for most pulsars (Kijak \& Gil, 1997).

Gangadhara \& Gupta (2001) and Dyks et al. (2004) introduced a phase-shift method to determine emission altitudes from core and conal emission components. The core component originates close to the surface and the conal component from a higher altitude. With this difference in emission altitude, the leading conal component should be more widely separated from the main pulse than the trailing conal component due to aberration and retardation effects introduced by the pulsar rotation. Under this assumption, the emission height can be calculated from the phase shifts of these leading and trailing components. Dyks et al. (2004) applied this method for several pulsar and found that the radio emission altitudes are $<100$ NS radii in general. 


\subsection{Pulsar timing}

Pulsars are highly accurate clocks. If we know the pulse period, period derivative, position, DM, and the proper motion of a solitary pulsar precisely, we can predict the pulse arrival of the pulsar to the Earth at any time in the future. In order to do this, we need a model which includes the spin properties of the pulsar and its astrometric information, so-called timing model. The pulse arrival at the Earth is recorded with respect to the observatory and then converted to the solar system barycenter by correcting for the Earth's orbital motion including the effect of other objects in the solar system (e.g., Romer delay corrects the Earth orbital motion, Shapiro delay corrects the delay due to space-time curvature in the solar system, and Einstein delay corrects the gravitational redshift and time dilation).

To obtain a timing model, first we need to measure times of arrival (TOAs) of observed pulses. The TOA is usually defined as the arrival time of some fiducial point (e.g. the pulse peak) on the mean pulse profile. We determine TOAs accurately by cross-correlating the observed profile with a high signal-to-noise template profile typically obtained from the addition of many earlier profiles at the same observing frequency. In order to time a pulsar, we usually obtain TOAs by observing it weekly or monthly. Then the model-predicted phase of the pulse $\phi(T)$ as a function of the barycentric time $T$ is given through Taylor expansion as

$$
\phi(T)=\phi_{0}+\left(T-T_{0}\right) \Omega_{0}+\frac{1}{2}\left(T-T_{0}\right)^{2} \dot{\Omega}_{0}+\ldots
$$

where $T_{0}$ is some reference epoch, $\Omega_{0}$ is the modeled rotational frequency and $\phi_{0}$ 
is the phase at epoch $T_{0}$. Then we fit the observed pulse arrival times to modelpredicted arrival times based on the initially estimated period, period derivative, position, and DM. Then we calculate the timing residuals (i.e., the difference between the observed and predicted pulse arrival times). The model is fit and the above parameters estimated in order to minimize the residuals. Using this model, we can predict the pulse phase in a future observation. With long-term observation of a pulsar, the timing precession can be significantly improved. Based on almost nine years of data of MSP B1937+21, the period measured by pulsar timing is $1.5578064688197945 \pm 0.0000000000000004 \mathrm{~ms}$ defined at midnight UT on December 5, 1988 (Kaspi et al., 1994). This is a good example of the stability of pulsar spin.

\subsection{Pulsars in binary systems}

If a pulsar is in a binary with a companion star, then we can constrain more physical properties than if it is isolated. Binary pulsars are important in studies of relativistic physics due to their tight orbits with strong gravitational fields. The first known binary pulsar system is PSR B1913+16, which is a pulsar orbiting another NS (Hulse \& Taylor, 1975) in a period of $7.75 \mathrm{hr}$. This system provides the first evidence of the existence of gravitational waves (GWs). Only ten double neutron star (DNS) systems have been discovered so far (Stairs, 2008; Keith et al., 2009; Kramer, 2010). 


\subsubsection{Timing binary pulsars}

In addition to the basic timing model of an isolated pulsar, there is more information that needs to be included in the timing model of a binary pulsar. We basically need to account for the rotational motion of the pulsar around the binary orbit. All binary parameters are modeled with respect to the barycenter of the binary system, so that it is easy to develop the timing solution. In order to model TOAs with respect to the barycenter by using Kepler's laws, there are five additional parameters required in the timing model; orbital period $P_{b}$, projected semi-major orbital axis $x$, orbital eccentricity $e$, longitude of periastron $\omega$, and epoch of periastron passage $T_{0}$. These five parameters are known as "Keplerian parameters" and can be constrained from pulsar timing. Then we determine the mass function from these parameters. We write the mass function in terms of the pulsar mass $\left(m_{\mathrm{p}}\right)$ and the companion mass $\left(m_{\mathrm{c}}\right)$,

$$
f_{\text {mass }}=\frac{4 \pi^{2} x^{3}}{G P_{b}^{2}}=\frac{\left(m_{c} \sin i\right)^{3}}{\left(m_{p}+m_{c}\right)^{2}}
$$

where $G$ is the Newton's gravitational constant and $i$ is the orbital inclination. With only Keplerian parameters, we can constrain $f_{\text {mass }}$ of the system through timing, but not individual masses and the orbital inclination.

However, some binary systems exhibit relativistic effects that require additional "post-Keplerian parameters" (PK) to model the system. According to GR, Blandford \& Teukolsky (1976) showed that the PK parameters are: the relativistic advance of periastron 


$$
\dot{\omega}=3\left(\frac{P_{b}}{2 \pi}\right)^{-5 / 3}\left(T_{\odot} M\right)^{2 / 3}\left(1-e^{2}\right)^{-1}
$$

the time dilation and gravitational redshift parameter

$$
\gamma=e\left(\frac{P_{b}}{2 \pi}\right)^{-1 / 3} T_{\odot}^{2 / 3} M^{-4 / 3} m_{c}\left(m_{p}+2 m_{c}\right)
$$

the rate of orbital decay due to gravitational radiation

$$
\dot{P}_{b}=-\frac{192 \pi}{5}\left(\frac{P_{b}}{2 \pi}\right)^{-5 / 3}\left(1+\frac{73}{24} e^{2}+\frac{37}{96} e^{4}\right)\left(1-e^{2}\right)^{-7 / 2} T_{\odot}^{5 / 3} m_{p} m_{c} M^{-1 / 3},
$$

and the two Shapiro delay parameters

$$
r=T_{\odot} m_{c}
$$

and

$$
s=\sin i=x\left(\frac{P_{b}}{2 \pi}\right)^{-2 / 3} T_{\odot}^{-1 / 3} M^{2 / 3} m_{c}^{-1}
$$

where, $M=m_{p}+m_{c}, x=a_{p} \sin i / c$, and $T_{\odot}=G M_{\odot} / c^{3}$. The two Shapiro delay parameters describe the pulse delay when the pulsar is at the superior conjunction where the radiation propagates through the gravitational well of the companion star. Note that these PK parameters depend on the masses of the pulsar and the companion and Keplerian parameters. 
As shown in the mass function (Equation 1.20), there are three unknowns $\left(m_{p}, m_{c}\right.$, and $\left.i\right)$. Therefore, by measuring two PK parameter along with the mass function, we are able to determine the individual masses and the orbital inclination. Measuring $\dot{\omega}$ and $\gamma$ for PSR B1913+16, Taylor \& Weisberg (1982) constrained the two masses for the first time. Taylor \& Weisberg (1989) reported the measurements of all five PK parameters of the system. Recently, Weisberg et al. (2010) analyzed more than three decades data on the system and obtained a better constraint for the pulsar mass $m_{p}=1.4398 \pm 0.0002 \mathrm{M}_{\odot}$ and the unseen companion mass $m_{c}=$ $1.3886 \pm 0.0002 \mathrm{M}_{\odot}$

Moreover, we can use measured PK parameters along with Keplerian parameter to test theories of GR (Damour \& Deruelle, 1986; Damour \& Taylor, 1992). The first binary pulsar used to test GR in this way was B1913+16. Taylor \& Weisberg (1989) obtained the orbital decay, which corresponds to a shrinkage of about $3.2 \mathrm{~mm}$ per orbit (see Figure 1.11) and this measurement agrees with the GR predicted one within $0.5 \%$ accuracy, provided the first indirect evidence for the existance of GWs. Hulse and Taylor were awarded the 1993 Nobel Physics prize for the discovery of this remarkable system for testing GR.

\subsubsection{Relativistic spin precession and radio emission variability in DNSs}

The total orbital angular momentum of a DNS is much larger than the individual angular momenta of the two NSs in the system. Therefore, the orbital angular 


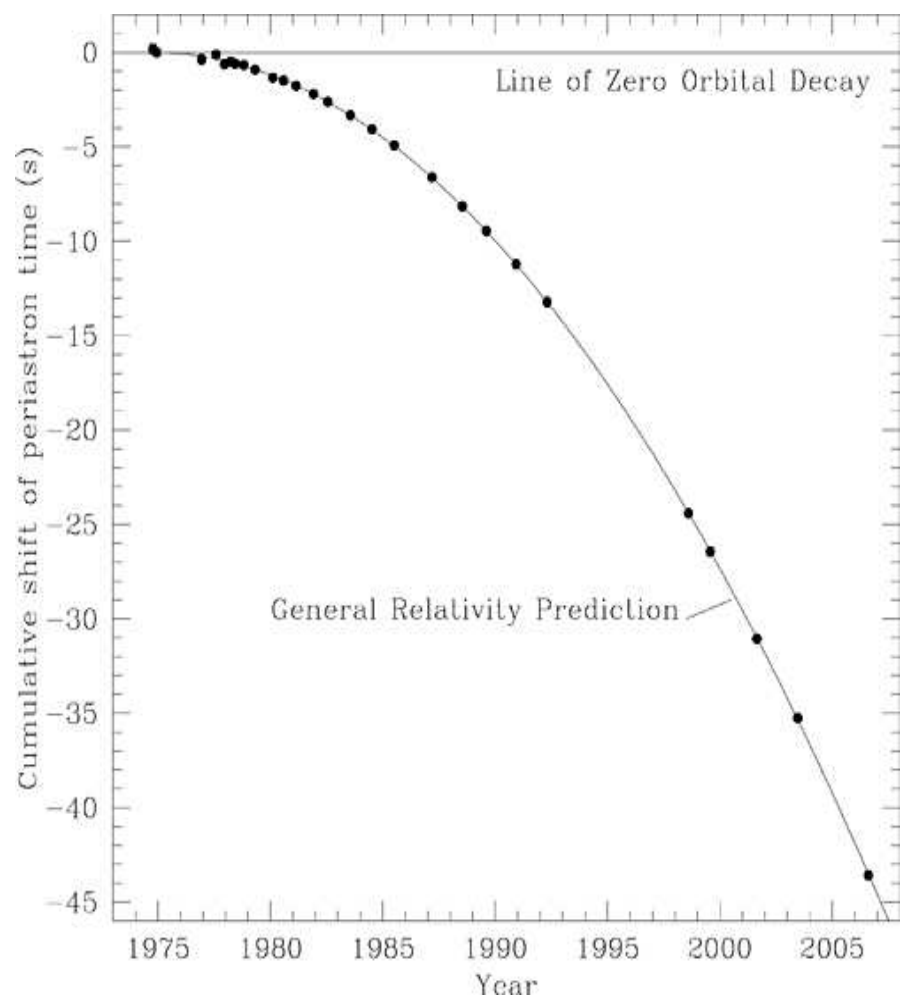

Figure 1.11: Orbital decay of PSR B1913+16 as reported in Weisberg et al. (2010). The dots are the measurements and the thick line is the GR predicted value.

momentum has a fixed direction in space. When the two NSs orbit each other in a tight binary, relativistic spin precession can occur due to strong gravitational fields. This forces the spin axis of the NS to precess around the orbital angular momentum axis. If the colatitude of the spin axis (i.e. the angle between the spin axis and the orbital angular momentum) is non-zero, the viewing geometry of the pulsar changes with spin precession. Therefore, the relative position of the radio beam with respect to our line of sight changes with time. Consequently, we detect modulation in the observed pulse profile properties, namely its shape and flux density. The spin precession is indeed measured for two binary systems through observation: for PSR J0737-3039B from a detailed study of J0737-3039A eclipses (Breton et al., 2008) and for PSR B1534+12 from the observed secular and periodic variations in pulse 
profiles due to spin precession and aberration (Stairs et al., 2004).

The theoretical equation formulated by Barker \& O'Connell (1975) and Boerner et al. (1975) shows that the predicted spin precession rate based on GR, known as geodetic spin precession, is

$$
\Omega_{\mathrm{prec}}=\frac{1}{2}\left(\frac{G M_{\odot}}{c^{3}}\right)^{2 / 3}\left(\frac{P_{\mathrm{b}}}{2 \pi}\right)^{-5 / 3} \frac{m_{\mathrm{c}}\left(4 m_{\mathrm{p}}+3 m_{\mathrm{c}}\right)}{\left(1-e^{2}\right)\left(m_{\mathrm{p}}+m_{\mathrm{c}}\right)^{4 / 3}}
$$

where $m_{\mathrm{p}}$ and $m_{\mathrm{c}}$ are the masses of the pulsar and its companion in units of the solar mass $M_{\odot}, P_{\mathrm{b}}$ is the orbital period, and $e$ is the eccentricity. Table 1.1 shows the known DNS systems so far with their properties and spin precession rates. Because the spin precession rates are comparably small for most of these pulsars, the secular profile variations are long-term. Based on geodetic spin precession, simple geometry models have been proposed to explain the pulse profile evolution of most of these systems.

As reported in Weisberg \& Taylor (2002), the pulse profile shape of PSR B1913+16 significantly evolved over the last 20 years. By fitting a circular beam geometrical model based on geodetic spin precession to the observed variation in pulse profile widths, the geometry angles $\alpha$ and $\beta$ of the pulsar were constrained (Weisberg \& Taylor, 2002; Clifton \& Weisberg, 2008). This is a very useful technique to constrain the geometry of precessing pulsars if the precession rate is large enough to detect the profile variation compared to the observing time. Also, this method can be used if the pulsar is not linearly polarized enough to use the RVM to determine the geometry (see Section 1.4). We use a similar method in Section 2.3 to constrain 
Table 1.1: Properties of known DNS systems sorted according to the expected relativistic spin precession rate (Kramer, 2010). Pulsar marked with an asterisk have had detected spin precession. For sources where no precession rate is listed, the companion mass has not been accurately measured yet.

\begin{tabular}{lcccc}
\hline \multicolumn{1}{c}{ Pulsar } & $\mathrm{P}(\mathrm{ms})$ & $\mathrm{P}_{\mathrm{b}}$ (days) & $e$ & $\Omega_{\text {prec }}\left({ }^{\circ} / \mathrm{yr}\right)$ \\
\hline J0737-3039A/B* & $22.7 / 2770$ & 0.10 & 0.09 & $4.8 / 5.1$ \\
J1906+0746* & 144.1 & 0.17 & 0.09 & 2.2 \\
$\mathrm{~B}^{*} 127+11 \mathrm{C}^{*}$ & 30.5 & 0.34 & 0.68 & 1.9 \\
$\mathrm{~B} 1913+16^{*}$ & 59.0 & 0.33 & 0.62 & 1.2 \\
$\mathrm{~J} 1756-2251$ & 28.5 & 0.32 & 0.18 & 0.8 \\
$\mathrm{~B} 1534+12^{*}$ & 37.9 & 0.42 & 0.27 & 0.5 \\
$\mathrm{~J} 1829+2456$ & 41.0 & 1.18 & 0.14 & 0.08 \\
$\mathrm{~J} 1518+4904$ & 40.9 & 8.64 & 0.25 & - \\
$\mathrm{J} 1753-2240$ & 95.1 & 13.63 & 0.30 & - \\
$\mathrm{J} 1811-1736$ & 104.2 & 18.8 & 0.83 & - \\
\hline
\end{tabular}

the geometry of PSR J0737-3039B. Furthermore, the geometries of binary pulsar systems PSRs B1534+12 (Stairs et al., 2000) and J1141-6545 (Manchester et al., 2010) were constrained using the same technique with their observed pulse profile variations (see Table 4.3).

The observed emission modulation depends not only on the precession rate, but on the geometry as well. For instance, PSR J0737-3039B shows a dramatic evolution in its pulse profiles, but J0737-3039A shows almost no variation even though it has a similar precession rate as of $\mathrm{B}$ (see Table 1.1). The reason for this is that J0737-3039A has spin axis that is nearly aligned with the orbital angular momentum (Ferdman et al., 2008, 2013). Therefore, the relative motion of the radio beam with respect to our line of sight is almost constant. In contrast, J0737-3039B has a larger spin-axis misalignment (Breton et al., 2008), so that the line of sight samples different cross sections of the radio beam, resulting in profile modulation. 
We present the pulse profile properties and the geometry of J0737-3039A/B in the next few chapters.

\subsection{Double pulsar system PSR J0737-3039A/B}

The detection of both NSs as radio pulsars is a rare situation, as it requires the electromagnetic beam from both pulsars to intercept our line of sight during the short interval when both pulsars are active. The only such system, PSR J0737-3039A/B, was discovered in late 2003 with the 64-m Parkes radio telescope in Australia as part of a high-latitude multibeam survey of the Southern sky (Burgay et al., 2003; Lyne et al., 2004). It provides an amazing laboratory for the study of relativistic gravity and the most precise test to date of GR in the strong-field regime (Kramer et al., 2006). The two pulsars orbit each other in a 2.4-hr orbit, the shortest of any observed DNSs, with moderate orbital eccentricity of 0.088 (see Table 1.1).

The first-born pulsar, hereafter 'A', is believed to have been recycled and therefore has a short spin period of $23 \mathrm{~ms}$. The second-born companion, hereafter 'B', spins with a longer $2.8 \mathrm{~s}$ period. The orbital plane of this system is almost edge-on to our line of sight. The orbital inclination was calculated to be $88.7^{\circ}$ through timing (Kramer et al., 2006) and $89.7^{\circ}$ through modeling of the interstellar scintillation properties of the two pulsars (Coles et al., 2005). Eclipses of A by B are a fortunate consequence of this favorable geometry. The 2.9 lt-s diameter of the orbit, determined through radio timing, and the $30 \mathrm{~s}$ duration of the eclipse show 
that the magnetosphere of B is roughly $10 \%$ of its $R_{\mathrm{LC}}$ (Lyne et al., 2004).

With strong gravitational fields and rapid motions of the two pulsars, the system is expected to show large relativistic effects and, indeed, has a relativistic advance of periastron $\dot{\omega}$ of $17^{\circ} \mathrm{yr}^{-1}$ (Kramer et al., 2006), the highest rate of any observed pulsar binary. McLaughlin et al. (2004a) studied the eclipses in detail and showed that there is an effect from pulsar B on the observed emission of pulsar A at the eclipse. Figure 1.12 shows the modulation in the pulsar A flux by pulsar B at the eclipse. During the eclipse, the light curve of A is modulated with the full and half spin period of B. Lyutikov \& Thompson (2005) shows that this modulation is due to absorption of A's radiation in the magnetosphere of pulsar B while spinning at a period of $2.8 \mathrm{~s}$. Based on this idea, Breton et al. (2008) constructed a model to explain the changing morphology of the eclipses of A and constrained the relativistic spin precession rate $\Omega_{\text {prec }}$ of pulsar B to be $4.8(7)^{\circ} \mathrm{yr}^{-1}{ }^{1}$. This estimate is consistent with the theoretical estimate of $\Omega_{\mathrm{prec}}=5.061(2)^{\circ} \mathrm{yr}^{-1}$ for B from Equation (1.26) within the errors. Furthermore, fitting for pulsar A eclipses, this model constrained the geometry of pulsar B; the magnetic misalignment and the spin colatitude are $\alpha_{\mathrm{B}}=70.9(4)^{\circ}$ and $\theta_{\mathrm{B}}=130.0(4)^{\circ}$, respectively (see Figure 1.13).

The predicted spin precession rate means that it takes 75 yrs for B's spin axis to complete a full precession cycle about the orbital angular momentum. This high precession rate is expected to have dramatic effects on B's pulse properties as a result of the change in apparent geometry with respect to our line of sight. These

\footnotetext{
${ }^{1}$ Here, and throughout the paper, the number in parentheses is the 1- $\sigma$ uncertainty in the last quoted digit.
} 


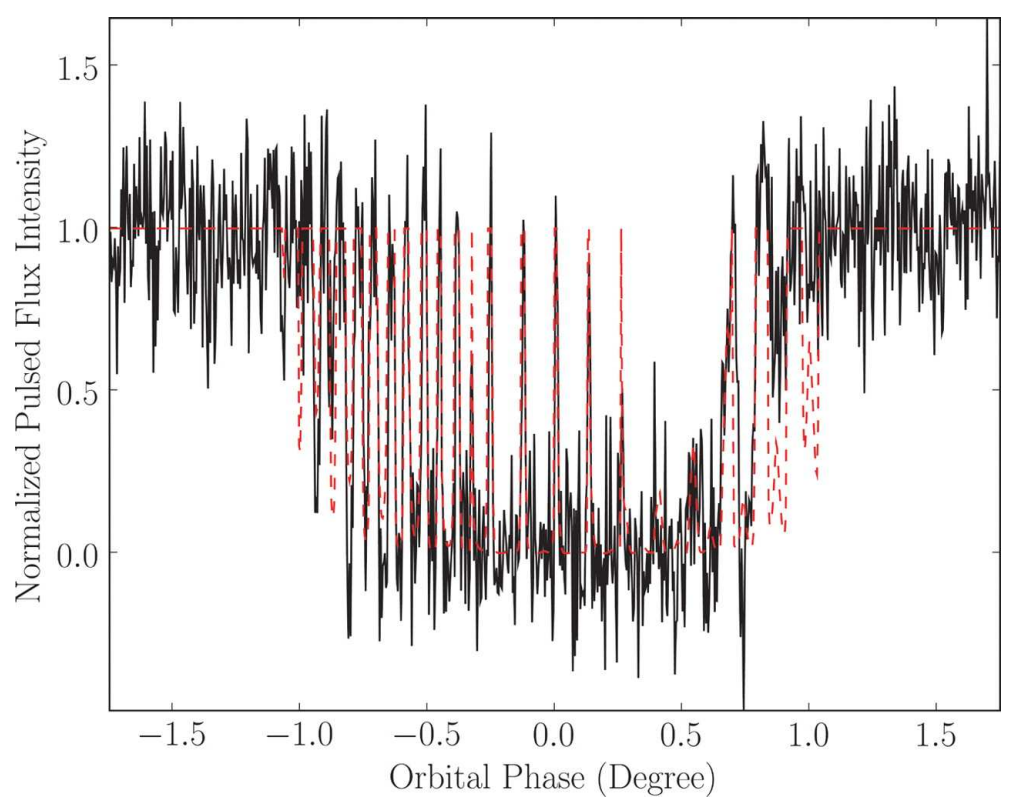

Figure 1.12: Modulation in observed pulsar A emission due to pulsar B at the eclipse. The pulsed flux is normalized to unity. The emission of A during the eclipse is modulated with the full and half spin periods of $\mathrm{B}$. The dashed line is the model predicted modulation (Breton et al., 2008).

include the evolution of its pulse profile, light curves, and flux densities (Burgay et al., 2005).

The more energetic pulsar A shows two distinct peaks in its pulse profile that are separated by $\sim 180^{\circ}$ in spin longitude (see Figure 4.1 ). The spin precession rate for pulsar $\mathrm{A}$ is calculated to be $4.772^{\circ} \mathrm{yr}^{-1}$ from Equation 1.26. Although the spin precession rates of $\mathrm{A}$ and $\mathrm{B}$ pulsars are comparable, there is no evidence for secular variation in pulse profiles of A (Manchester et al., 2005; Ferdman et al., 2008, 2013). The most plausible explanation for this stable pulse profile given the high spin precession rate is that pulsar A's spin misalignment, $\theta_{\mathrm{A}}$, from the orbit normal is very small. Assuming the two well separated $\left(\sim 180^{\circ}\right.$ in spin longitude) pulse components in the pulse profile are formed from a single-pole circular beam, Manchester et al. (2005) constrained $\theta_{\mathrm{A}} \leq 60^{\circ}$ and the magnetic misalignment 


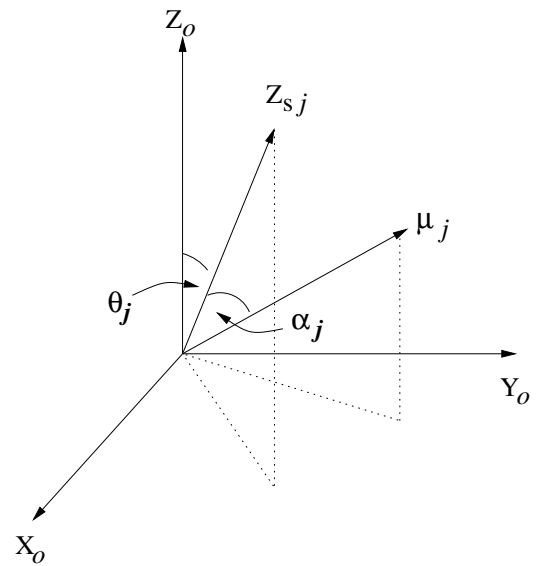

(a)

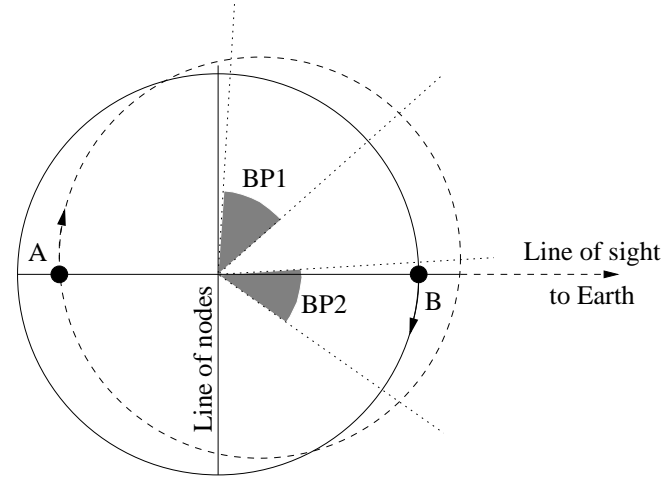

(b)

Figure 1.13: (a) The geometry of the pulsar in the orbital frame $\left(X_{o}, Y_{o}, Z_{o}\right)$. The subscript $j$ represents pulsar A or pulsar B. The spin axis and the magnetic axis are shown by $Z_{s}$ and $\mu$, respectively. The spin colatitude $\theta$ is the angle between the orbital normal $Z_{o}$ and the spin axis $Z_{s}$. The magnetic inclination $\alpha$ is the angle between the spin axis and the magnetic axis. (b) The schematic diagram of the two orbits of pulsar A and B. The dashed and solid lines represent the orbits of pulsar $\mathrm{A}$ and pulsar $\mathrm{B}$, respectively. The orbital longitude is measured with respect to the ascending node. The two BPs of pulsar B $\left(\sim 185^{\circ}-235^{\circ}\right.$ and $\left.\sim 265^{\circ}-305^{\circ}\right)$ are shaded.

$\alpha_{\mathrm{A}} \sim 19^{\circ}$ with a broad beam (i.e., a half-opening angle of $\rho_{\mathrm{A}} \sim 90^{\circ}$ ). We note that the high-energy outer magnetosphere geometry models are also capable of producing broad fan-like emission beams and about $180^{\circ}$ peak separations in the pulse profiles due to their caustic regions. However, as mentioned in Section 1.3.2, the radio emission generation from the outer magnetosphere is not well established except for a few pulsars such as the Crab pulsar and PSR B1937+21, where the radio and gamma-ray peaks are in close alignment, implying that both the radio and the highenergy emission are generated within the outer magnetosphere (Cheng \& Ruderman, 1977; Moffett \& Hankins, 1996).

Assuming the two pulse components in the profile are formed from the two radio beams corresponding to each magnetic pole of pulsar A, Ferdman et al. (2008, 
2013) determined its geometry with a double-pole circular beam model. By analyzing six years of data, Ferdman et al. (2013) found that the pulse profile widths of pulsar A show no significant variation with time. By fitting a double-pole circular beam model to measured pulse profile widths at different intensity levels (30\%, $35 \%, 40 \%, 45 \%$, and $50 \%$ with respect to the peak) individually, they constrained the pulsar geometry to be $\alpha_{\mathrm{A}}=90^{\circ} \pm 16^{\circ}$ and $\theta_{\mathrm{A}}<5^{\circ}$ at a $95 \%$ confidence level. This constrained geometry supports the orthogonal configuration of pulsar A.

Recent Fermi observations of the Double Pulsar detected pulsed gamma-ray emission from pulsar A (Guillemot et al., 2013). The high-energy gamma-ray emission is explained by OG and TPC models (see Section 1.3.2). They constrained pulsar A's geometric angles $\alpha_{\mathrm{A}}$ and $\zeta_{\mathrm{E}}$, where $\zeta_{\mathrm{E}}$ is the viewing angle of the line of sight (i.e., the angle between pulsar A's spin axis and our line of sight), by fitting the OG and the TPC models separately to observed gamma-ray light curves. As an independent estimate, they also used radio polarization measurements to constrain these two angles from the RVM (see Section 1.4). Moreover, they found that the peaks of the gamma-ray and the radio profiles are not aligned. This implies that the radio and gamma-ray emission are generated in different locations in pulsar A's magnetosphere. Therefore, it is evident that pulsar A's radio emission originates within the inner magnetosphere, so that the preferred pulsar radio beam geometry is double-pole. Thus, we adopt this configuration in Chapter 4.

The high spin-down energy loss rate of A likely causes the magnetosphere of B to be deformed in a way similar to that of the Earth by the Solar wind (Lyutikov, 2004). The long tail will point towards the Earth around the eclipse of A by B, near 
Table 1.2: Starting and ending orbital longitudes of each phase in which B is easily detected.

\begin{tabular}{lllll}
\hline & BP1 & BP2 & WP1 & WP2 \\
\hline Start & $185^{\circ}$ & $265^{\circ}$ & $340^{\circ}$ & $80^{\circ}$ \\
End & $235^{\circ}$ & $305^{\circ}$ & $30^{\circ}$ & $130^{\circ}$ \\
\hline
\end{tabular}

(The two bright phases are denoted as BP1 and BP2 and the two weak phases as WP1 and WP2)

the inferior conjunction of B (i.e. at an orbital phase of $270^{\circ}$, as measured from the ascending node, see Figure 1.13). Lyne et al. (2004) finds that the bright emission from $\mathrm{B}$ is detected in two different orbital phase windows: at $\sim 185^{\circ}-235^{\circ}$ (hereafter BP1), and $\sim 265^{\circ}-305^{\circ}$ (hereafter BP2); see Figure 1.13 for the illustration of the two BPs in the orbit. Table 1.2 shows the longitudes (as measured from the ascending node) of each phase in which B is easily detected in early observations.

Figure 1.14 shows actual data of the two bright phases on MJD 52997 (December 24, 2003). In addition to these two bright phases, the pulsar often shows weak emission at other orbital phases (Lyne et al., 2004). The exact explanation of this exceptional behavior is challenging. The model of Lyutikov (2005) explains the phenomenon generally, but fails to produce the second bright phase at the correct orbital phase $\left(\sim 245^{\circ}-270^{\circ}\right.$ instead of $\left.\sim 260^{\circ}-310^{\circ}\right)$. Further, the model was unable to explain the observed evolution of the two bright phases over time.

Another effect of A's electromagnetic radiation on B's magnetosphere is drifting subpulses in the emission of $\mathrm{B}$ which appear similar to those seen for some normal radio pulsars (McLaughlin et al., 2004b). The drifting feature can be seen only in BP1 and not in BP2. The separation of the driftbands is equal to the period 


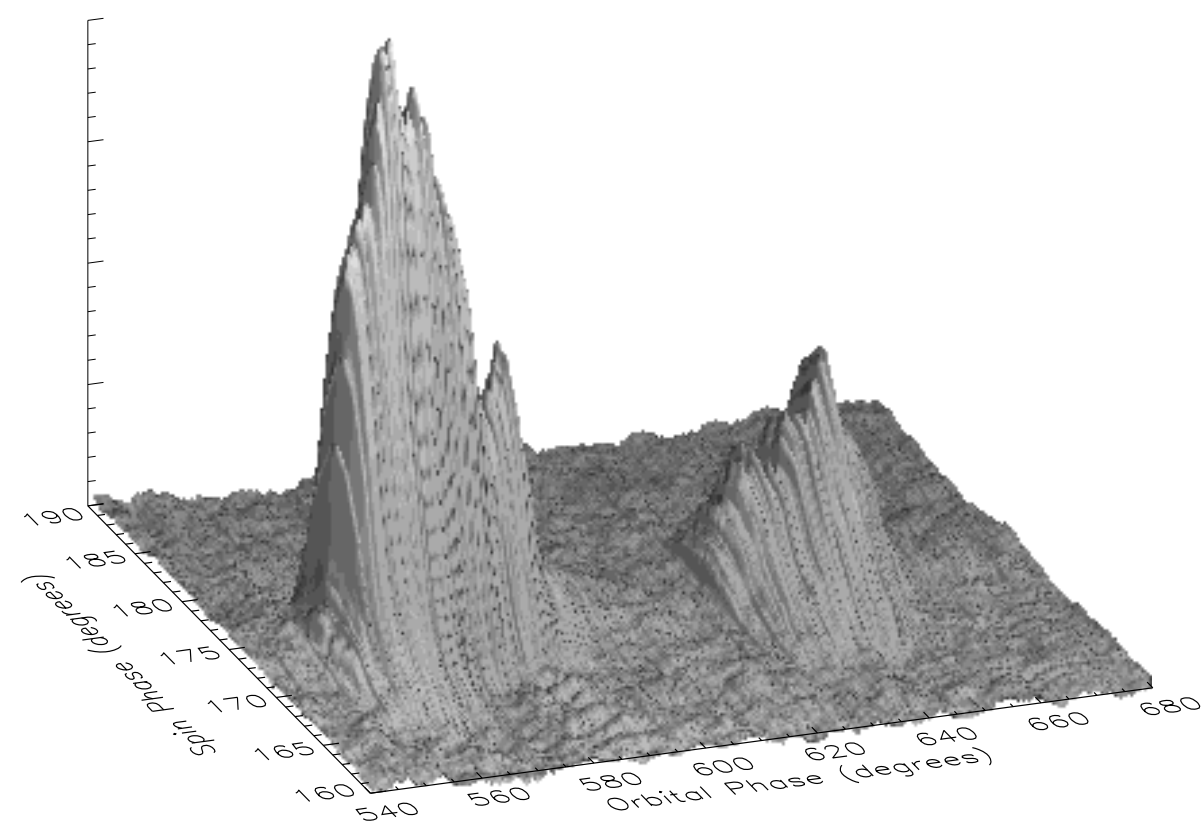

Figure 1.14: The observed radio emission of the two BPs of pulsar B in 3-D view on MJD 52997 (December 24, 2003). The axes are orbital and spin phases in degrees and the height is the intensity.

of A, showing that the drifting features are a direct result of the impact of A's 44 $\mathrm{Hz}$ electromagnetic radiation on $\mathrm{B}$ (see Figure 1.15). A possible explanation for the occurrence of drifting features only in BP1 is the particular geometric configuration of the two pulsars with respect to our line of sight. Freire et al. (2009) proposed a new technique for timing the double pulsar system by describing a geometrical model which predicts the delay of B's subpulses relative to A's radio pulses.

This system is very important in testing general relativity. As described in Section 1.7.1, binary systems can be used to determine the mass function from Keplerian parameters. This system also provides the calculation of the mass function. Since pulsar A is more stable, all five of its measured PK parameters along with pulsar B's measured projected semi-major axis used to determine the masses 

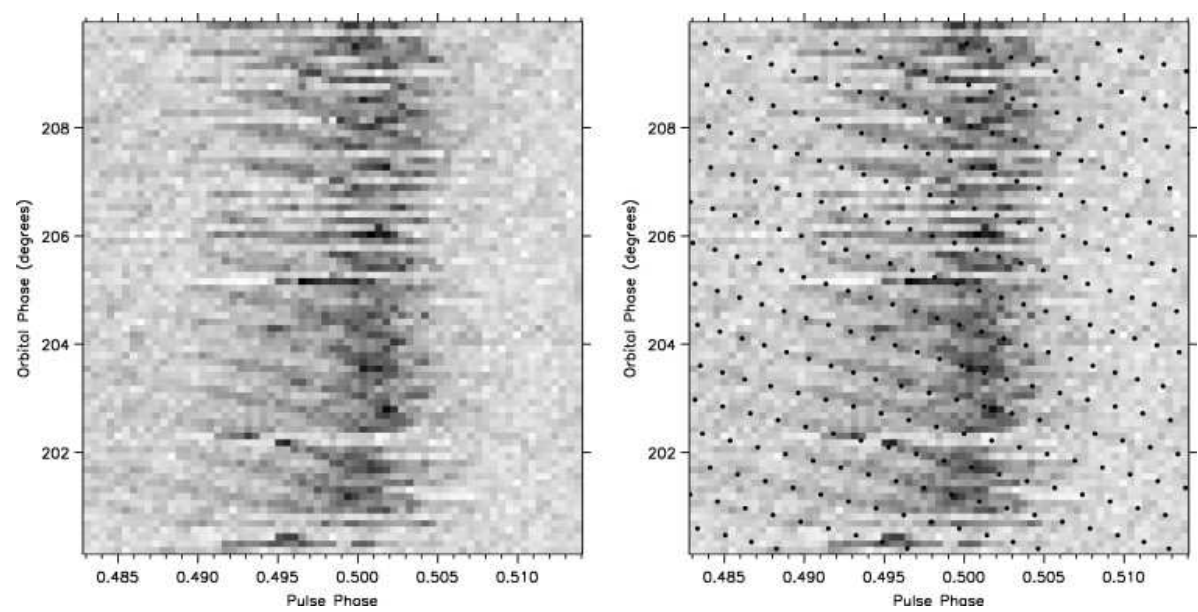

Figure 1.15: Drifting sub-pulses of pulsar B in BP1. Left: Single pulses of B at 820 $\mathrm{MHz}$ observing frequency for BP1. Note that the drifting feature is clearly seen. Right: Dots denote the emission from an arbitrary rotational phase of A arriving at the center of B (McLaughlin et al., 2004b).

of the system. The projected semi-major axes of both orbits gives the mass ratio $R=1.071 \pm 0.001$. Therefore, by using $R$ and one PK parameter with the mass function, Kramer et al. (2006) constrained the individual masses of the two pulsars as $m_{A}=1.3381 \pm 0.0007 \mathrm{M}_{\odot}$ and $m_{B}=1.2489 \pm 0.0007 \mathrm{M}_{\odot}$ (see Figure 1.16). Then they used the other four PK parameters to compare GR-predicted values with the measured ones for given masses, resulting in four different tests for GR. The most precise test was the PK parameter Shapiro delay $s$ which agrees with the value predicted by GR within an uncertainty of $0.05 \%$. This is the most precise test for GR yet obtained for a binary system. The measured $\dot{P}_{b}$ means a shrinkage of the orbit of $7 \mathrm{~mm}$ per day. 


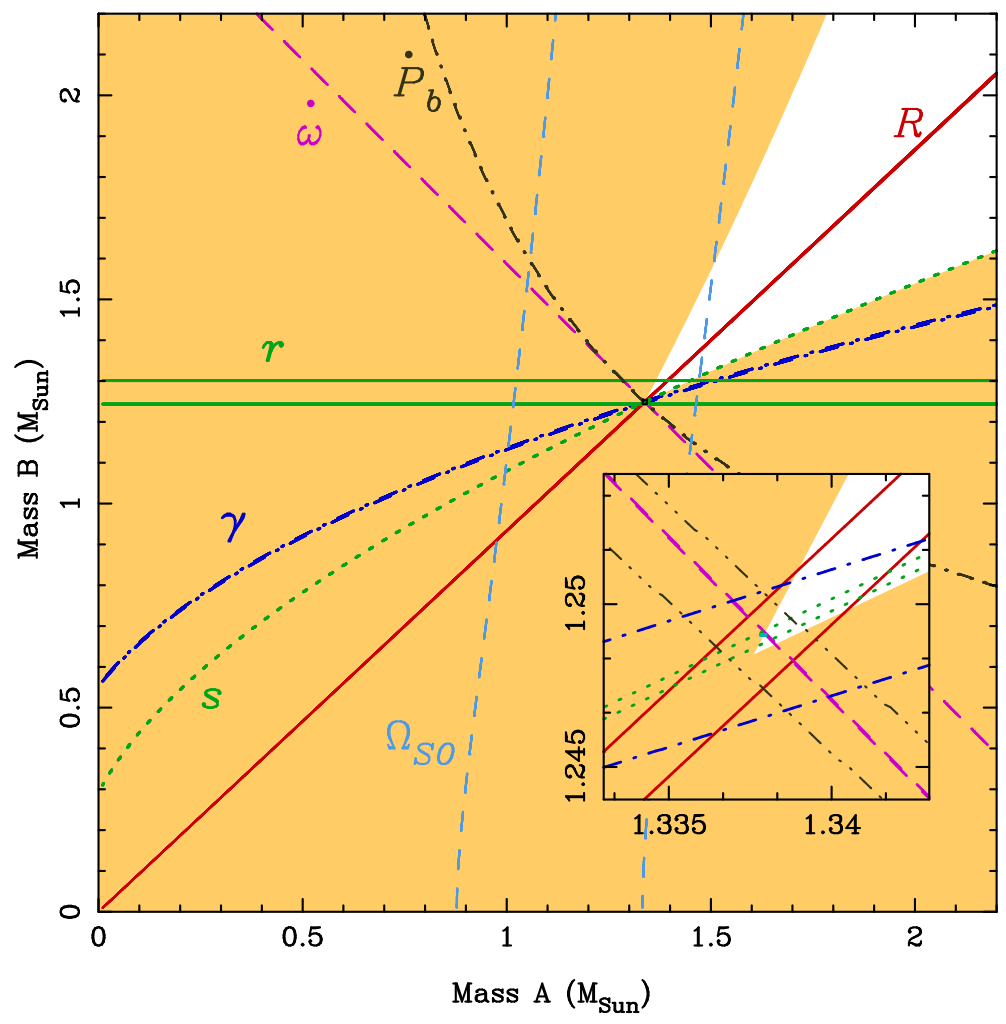

Figure 1.16: Mass-mass plot for the double pulsar system by using four different tests of GR. The shaded regions are forbidden by the individual mass functions of $\mathrm{A}$ and $\mathrm{B}$ due to the fact that $\sin i \leq 1$. The constraining parameters are shown with pairs of lines, where the separation of lines indicates the uncertainty measurement (private communication with Michael Kramer). 


\section{Chapter 2}

\section{Radio emission evolution and the geometry of J0737-3039B}

This chapter presents the dramatic evolution in observed radio emission properties of PSR J0737-3039B (pulsar B). We analyze B's pulse profile and mean flux density variation over time based on more than five years of data. Then we report the radio emission disappearance from pulsar B towards our line of sight since 2008 March. From a geometric model based on geodetic spin precession, we explain the observed profile evolution and then constrain the geometry of pulsar B.

Most of the work in this chapter was originally published as Perera B. B. P., McLaughlin M. A., Kramer M., Stairs I. H., Ferdman R. D., Freire P. C. C., Possenti A., Breton R. P., Manchester R. N., Burgay M., Lyne A. G., Camilo F., 2010, ApJ, 721, 1193; The Evolution of PSR J0737-3039B and a Model for Relativistic Spin Precession

\subsection{Introduction}

Some of the DNSs show changes in their observed radio emission properties over time. Among these systems, PSRs B1913+16 (Weisberg et al., 1989; Kramer, 1998; Weisberg \& Taylor, 2005), B1534+12 (Arzoumanian, 1996; Stairs et al., 2004),

J1141-6545 (Hotan et al., 2005; Manchester et al., 2010), and J1906+0746 (Lorimer 
et al., 2006a) show pulse profile evolution. These observed changes in pulse profiles can be explained through geodetic spin precession as described in Section 1.7.2.

Similarly, Burgay et al. (2005) reported that pulsar B shows evolution in observed radio pulse emission with time. Analyzing 20 months of data, they showed that the shape of the pulse profile and the pulse intensity across the orbit vary with time. This variation is somewhat short-term and dramatic when compared to the profile variation of other binary pulsars. This is likely due to the high spin precession rate, $5.1^{\circ} \mathrm{yr}^{-1}$, of pulsar $\mathrm{B}$.

\subsection{Observations}

We observed J0737-3039B with the 100-m Green Bank Telescope (GBT) in West Virginia from 2003 December 24 to 2009 June 20. Our observations are at multiple frequencies - because $820 \mathrm{MHz}$ is the most common, we use only those data for this analysis. Until 2009 January, the data were taken using the GBT spectrometer SPIGOT card with sampling time of $81.92 \mu \mathrm{s}$. SPIGOT provides 1024 synthesized frequency channels across a $50 \mathrm{MHz}$ bandwidth. After 2009 January, the GBT spectrometer GUPPI was used. It covers 2048 frequency channels with sampling time of $61.44 \mu \mathrm{s}$, with a wider bandwidth of $200 \mathrm{MHz}$. All the data were dedispersed and folded using the pulsar analysis package SIGPROC, assuming a DM of $48.914 \mathrm{~cm}^{-3}$ pc (Lyne et al., 2004). We used the ephemeris of Lyne et al. (2004) until 2006, and since then have used the ephemeris of Kramer et al. (2006) to form mean pulse profiles. A total of 52 data sets with 234 total hours of observations at 
$820 \mathrm{MHz}$ have been analyzed.

\subsubsection{Pulse profile evolution of the two bright phases}

As mentioned in Section 1.8, pulsar B is brightest in the orbital phase region $185^{\circ}-235^{\circ}(\mathrm{BP} 1)$, and second brightest in $265^{\circ}-305^{\circ}$ (BP2) (see Table 1.2 and Figure 1.14). In Figure 2.1 and 2.2, we present the integrated pulse profiles for BP1 and $\mathrm{BP} 2$, respectively, on 12 days that pulsar B was observed at $820 \mathrm{MHz}$. The very first observation, which was made in December 2003, shows a unimodal average pulse profile. This unimodal pulse profile gets broader and splits gradually into a two-component pulse profile over time. This evolution is common for both bright phases, but the specific features are somewhat different.

The evolution from a single-peak profile towards a two-peak profile begins around 2006 May (MJD 53860). In BP1, the first peak is brighter than the second one. This two-peak profile exists almost until the disappearance of the pulsar in this orbital phase region (see Figure 2.1). In BP2, the evolution has a similar pattern to $\mathrm{BP} 1$, but the remarkable difference is that the relative heights of the two peaks differ (see Figure 2.2). It initially looks similar to the profile of BP1, but on MJD 54055 the relative amplitudes of the two peaks change and the second one becomes more prominent. This persists throughout the evolution and we believe that this feature is due to the impact of A's emission on B, the effect of which changes over the period of the observation. Grey-scale plots of the first and the second bright phases of the two-peak profile are shown in Figure 2.3. The first peak of each epoch 


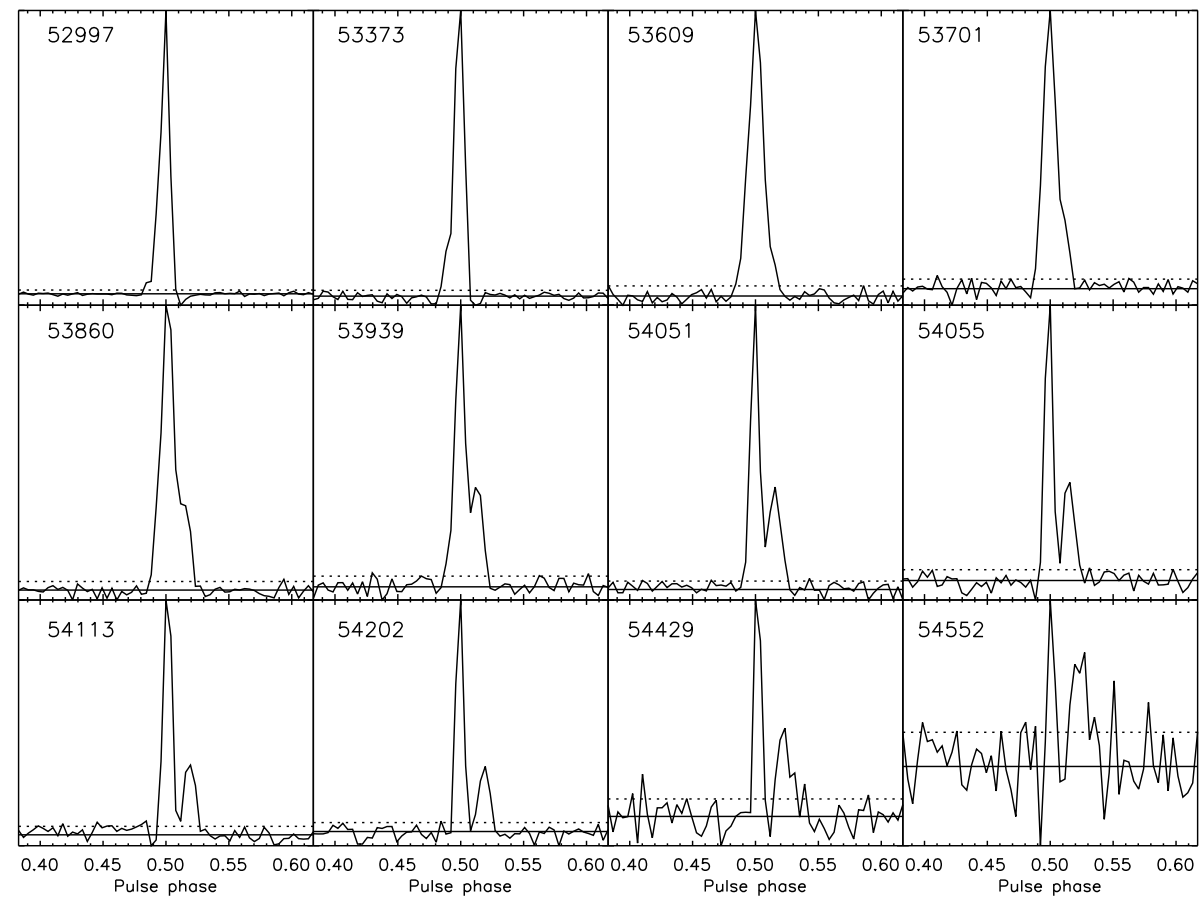

Figure 2.1: The pulse profiles of BP1 on 12 different days including the very first observation (MJD 52997). All data have been observed at a frequency of $820 \mathrm{MHz}$. Each profile covers 20 min of orbital longitude (from $185^{\circ}-235^{\circ}$ ) and there are 256 bins across the entire profile. Since predictions of absolute pulse phase are not available for these observations, the highest profile peak has been aligned to phase 0.5 at each epoch. The horizontal solid and dotted lines show the baseline, or offpulse mean, of the profile and the corresponding standard deviation of the off-peak region, respectively. The effective time resolution of the profiles is $10 \mathrm{~ms}$. 


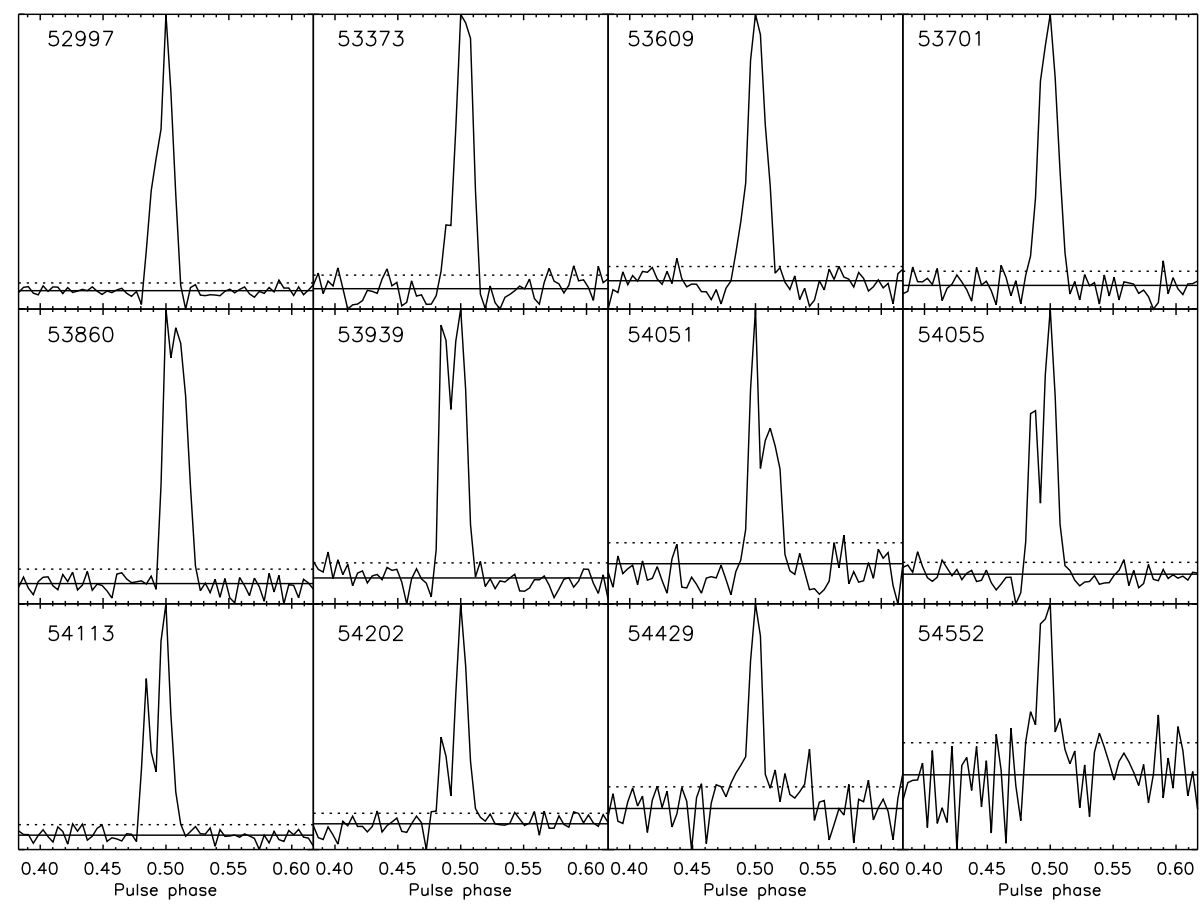

Figure 2.2: As described in Figure 2.1, but for BP2.

has been normalized and aligned in both phases. It can be clearly seen that the two peaks are moving apart and the separation grows with time in both phases simultaneously.

In order to get more information on the two peaks to describe the evolution, we fit two Gaussians separately for each peak of the two-peak pulse profile. From the Gaussian fits, we calculate the full-width-half-max (FWHM) of each peak of the two-peak pulse profiles in the two bright phases. They are shown in Figures 2.4 and 2.5. Linear least-squares fits qualitatively show that the FWHM of the first peak of BP1 decreases with time while that of the second peak increases slightly. In BP2, the FWHM of the first peak shows a slight decrease, but the decrease of second is more prominent.

We present the evolution of the separation of the peaks in the two bright phases 

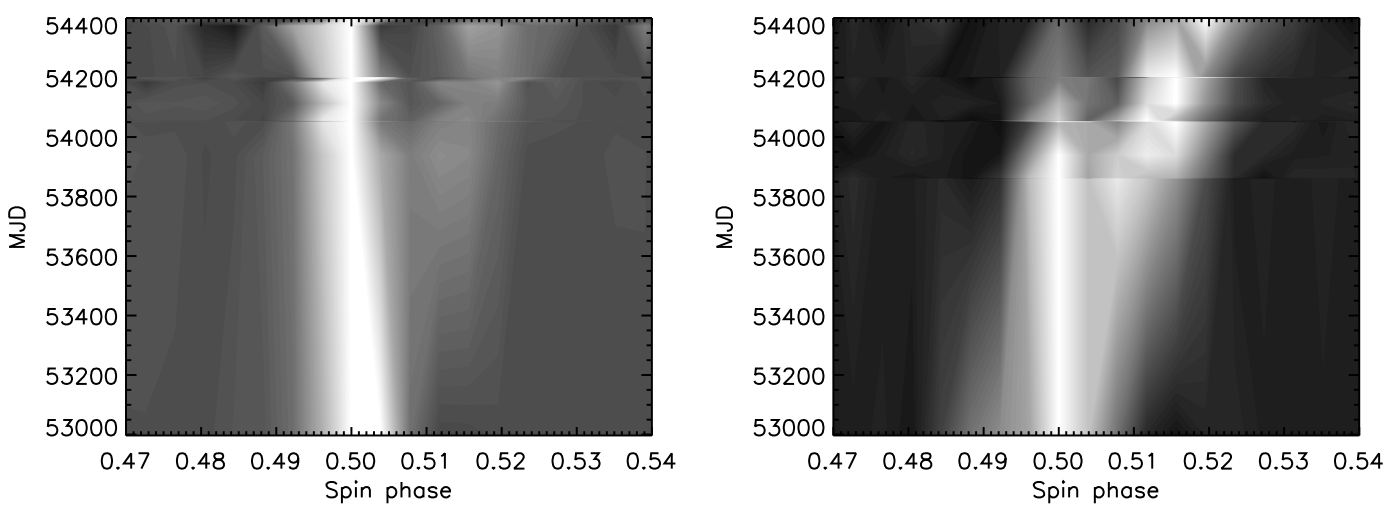

Figure 2.3: Grey-scale plots of BP1 (left) and BP2 (right). There are 256 bins across each pulse profile. The first peak of each pulse profile has been normalized to unity and aligned to visualize how the second peak evolves. The discontinuities of the plots are artifacts due to boundary level changes.
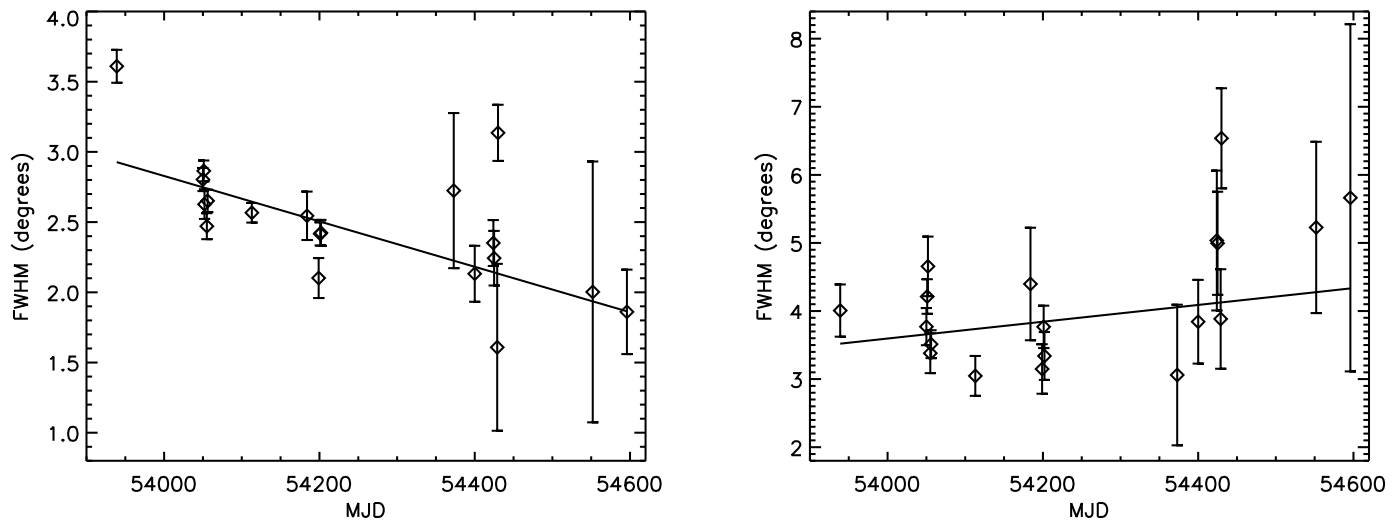

Figure 2.4: Full width at half max (FWHM) of the first peak of BP1 (left) and the second peak of BP1 (right). The linear least-squares fits show a decreasing FWHM rate of $0.59(8)^{\circ} \mathrm{yr}^{-1}$ for the first peak and increasing FWHM rate of $0.4(3)^{\circ} \mathrm{yr}^{-1}$ for the second peak. 

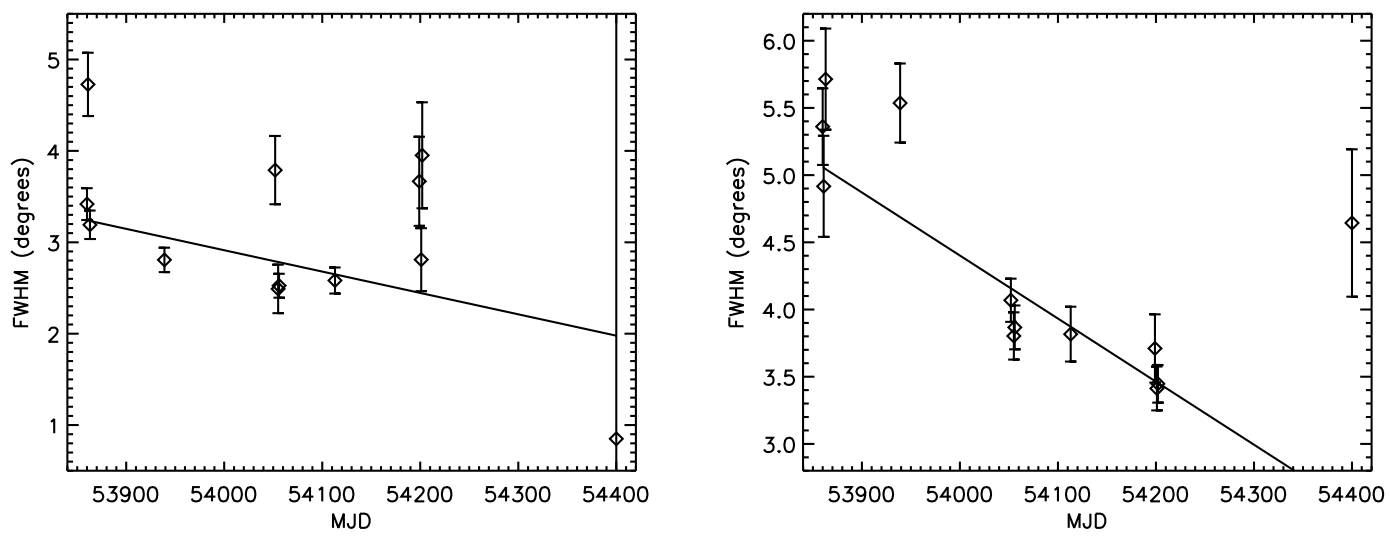

Figure 2.5: FWHM of the first peak of BP2 (left) and that of second peak of BP2 (right). The linear least-squares fits show a decreasing FWHM rate of $0.8(2)^{\circ} \mathrm{yr}^{-1}$ and $1.7(2)^{\circ} \mathrm{yr}^{-1}$ for the first and second peak, respectively. Note that due to the small number of bright pulses included in these composite profiles, the profiles show a high amount of variability that does not follow any trend.

in Figure 2.6. The rates of separation are calculated from a linear least-squares fit to be 2.6(1) ${ }^{\circ} \mathrm{yr}^{-1}$ and $2.6(2)^{\circ} \mathrm{yr}^{-1}$ for $\mathrm{BP} 1$ and $\mathrm{BP} 2$, respectively. It appears that the profiles in both bright regions present the same rate of change in their component separation.

\subsubsection{Flux evolution of the two bright phases}

In both bright regions, the integrated pulse flux density has decreased gradually over time (see Figure 2.1 and 2.2). The pulsar was detected in both bright phases with the last significant detection in March 2008 (MJD 54552) at $820 \mathrm{MHz}$.

We estimate flux densities at $820 \mathrm{MHz}$ using the radiometer equation. From the radiometer equation, the noise fluctuation in mJy is given by

$$
\Delta S_{\mathrm{sys}}=\frac{T_{\mathrm{sys}}}{G \sqrt{n_{\mathrm{p}} t_{\mathrm{obs}} \Delta f}}
$$



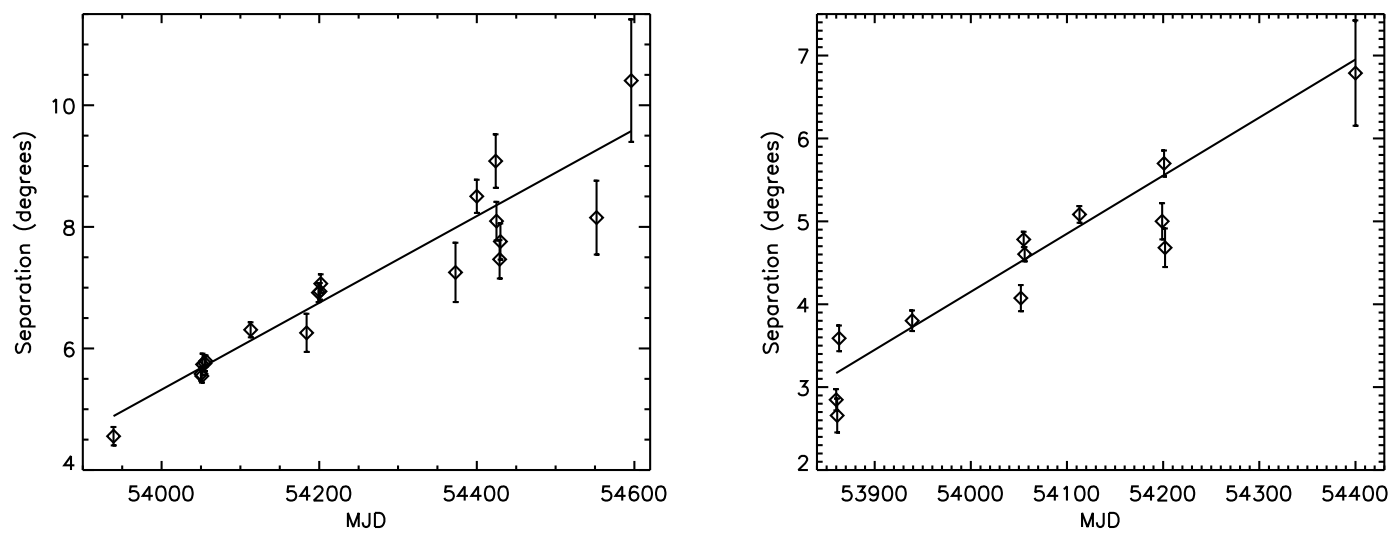

Figure 2.6: The varying separation of the two peaks of pulse profiles of BP1 (left) and BP2 (right). The linear least-squares fits show a separation rate of $2.6(1)^{\circ} \mathrm{yr}^{-1}$ and 2.6(2) ${ }^{\circ} \mathrm{yr}^{-1}$ for $\mathrm{BP} 1$ and $\mathrm{BP} 2$, respectively. Note that in BP1, the two-peak pulse profile lasts longer than in BP2.

where $T_{\text {sys }}$ is the system temperature $(\mathrm{K}), G$ is the telescope gain (which is $2 \mathrm{~K} / \mathrm{Jy}$ for the GBT), $n_{\mathrm{p}}$ is the number of polarizations summed (which is 2 in our case), $t_{\mathrm{obs}}$ is the observation length, and $\Delta f$ is the observing bandwidth $(\mathrm{MHz})$ (see Lorimer \& Kramer, 2005). The system temperature depends on the position in the sky and the observing frequency. For pulsar B at frequency $820 \mathrm{MHz}$, it is estimated to be $35 \mathrm{~K}$. First we calculate the radiometer noise and consider this as the flux density at the off-pulse region of the pulse profile. The flux density is then obtained by multiplying the pulse profile by the ratio of radiometer noise to the standard deviation of the off-pulse phase and subtracting the mean off-pulse level. We have carried out this calculation for only the two bright phases, because the emission in the weak phases disappeared much earlier (discussed in Section 2.2.4 with more details). The calculated flux densities in both bright phases on MJD 52997 (0.95(4) and 0.65(4) mJy for BP1 and BP2 at $820 \mathrm{MHz}$, respectively) are consistent with the value that has been calculated by Lyne et al. (2004) (0-1.3(3) mJy at $1390 \mathrm{MHz}$ ). 

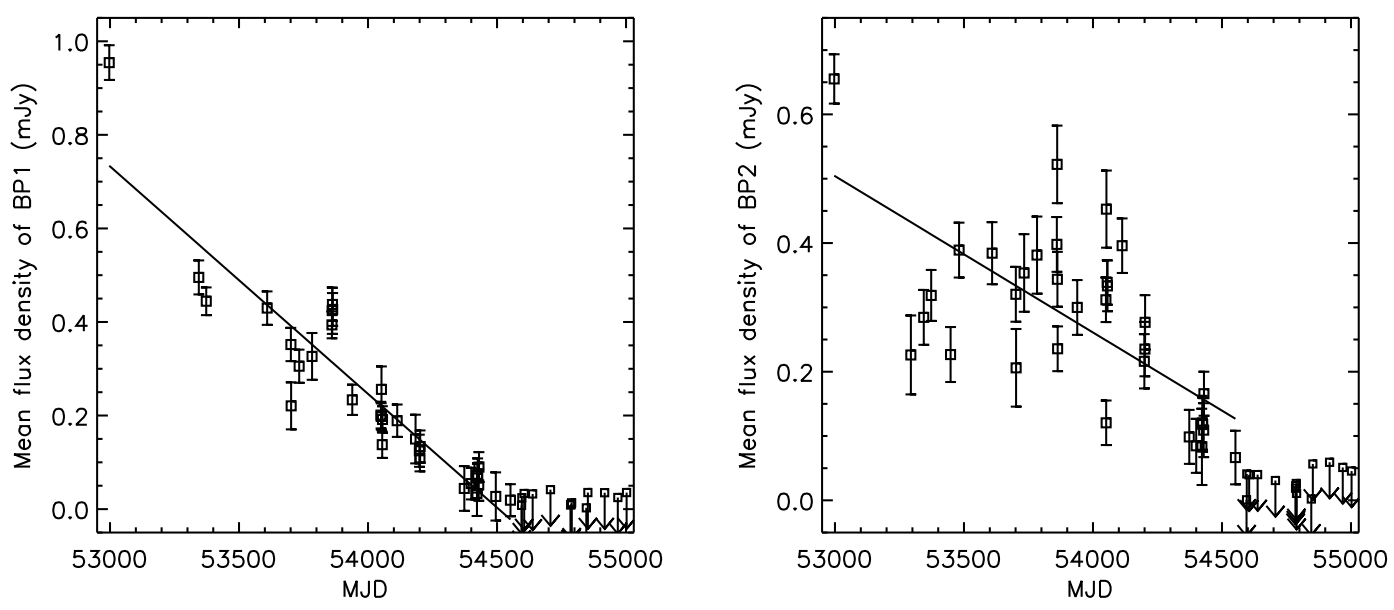

Figure 2.7: Mean flux density of the radio emission in BP1 (left) and in BP2 (right). The data marked by the arrows are the upper limits and ignored in our linear leastsquares fit. The decreasing rate of the mean flux density is calculated to be $0.177(6)$ and $0.089(7) \mathrm{mJy} \mathrm{yr}^{-1}$ for $\mathrm{BP} 1$ and $\mathrm{BP} 2$, respectively.

Figure 2.7 shows the mean flux densities of different epochs which have been observed at $820 \mathrm{MHz}$. This confirms that the flux density significantly decreases over time and almost reaches zero around MJD 54852 (2009 January) in both bright phases. The rate of decrease is calculated to be $0.177(6)$ and $0.089(7) \mathrm{mJy} \mathrm{yr}^{-1}$ for BP1 and BP2, respectively. The flux densities of the last few epochs are only upper limits (denoted by arrows) and not included in the fits. Our timing solution is not stable enough to provide a reliable prediction of the expected phase of the pulsar on these days, making it difficult to determine whether any apparent peaks are real. The peaks on MJDs 54856 and 54852 have the same pulse phase in both bright phases, suggesting they are real. However, given their low signal-to-noise, we describe them by upper limits. 


\subsubsection{Analysis and comparison of light curves of the two bright phases}

The orbital-phase binned light curves of the two bright phases were obtained by integrating the flux in a window covering $5 \%$ of the spin period and centered on the pulse peak to reduce the effect of baseline noise. Then each light curve is smoothed by using a boxcar with a width of 30 pulses to reduce the significant pulse-to-pulse modulation. In Figures 2.8 and 2.9, we present the light curves of the two bright phases on 12 different days that have been observed at frequency 820 MHz. Initially in both bright phases the emission was detected in a region covering $\sim 30^{\circ}$ of orbital phase, but this has shrunk over time, although this did not happen symmetrically in both phases.

In order to analyze the evolution of the two bright phases with time, we calculated the start and end orbital longitudes, at the $10 \%$ of the maximum, of $\mathrm{BP} 1$ and BP2. These are shown in Figure 2.10. The linear least-squares fits show the start of BP1 moves to higher longitudes at a rate of $3.1(4)^{\circ} \mathrm{yr}^{-1}$ and the end of $\mathrm{BP} 1$ moves backwards at a rate of $-1.4(4)^{\circ} \mathrm{yr}^{-1}$. In BP2, the both start and end orbital longitudes move to higher values at a rate of $4.1(2)^{\circ} \mathrm{yr}^{-1}$ and $2.1(2)^{\circ} \mathrm{yr}^{-1}$. These rates show that $\mathrm{BP} 1$ and $\mathrm{BP} 2$ shrink at a rate of $4.5(6)^{\circ} \mathrm{yr}^{-1}$ and $2.1(3)^{\circ} \mathrm{yr}^{-1}$, respectively. The above rates are updates for those presented by Burgay et al. (2005). These results confirmed that the disappearance did not happen symmetrically. However, the disappearance did occur simultaneously in both bright phase regions. 


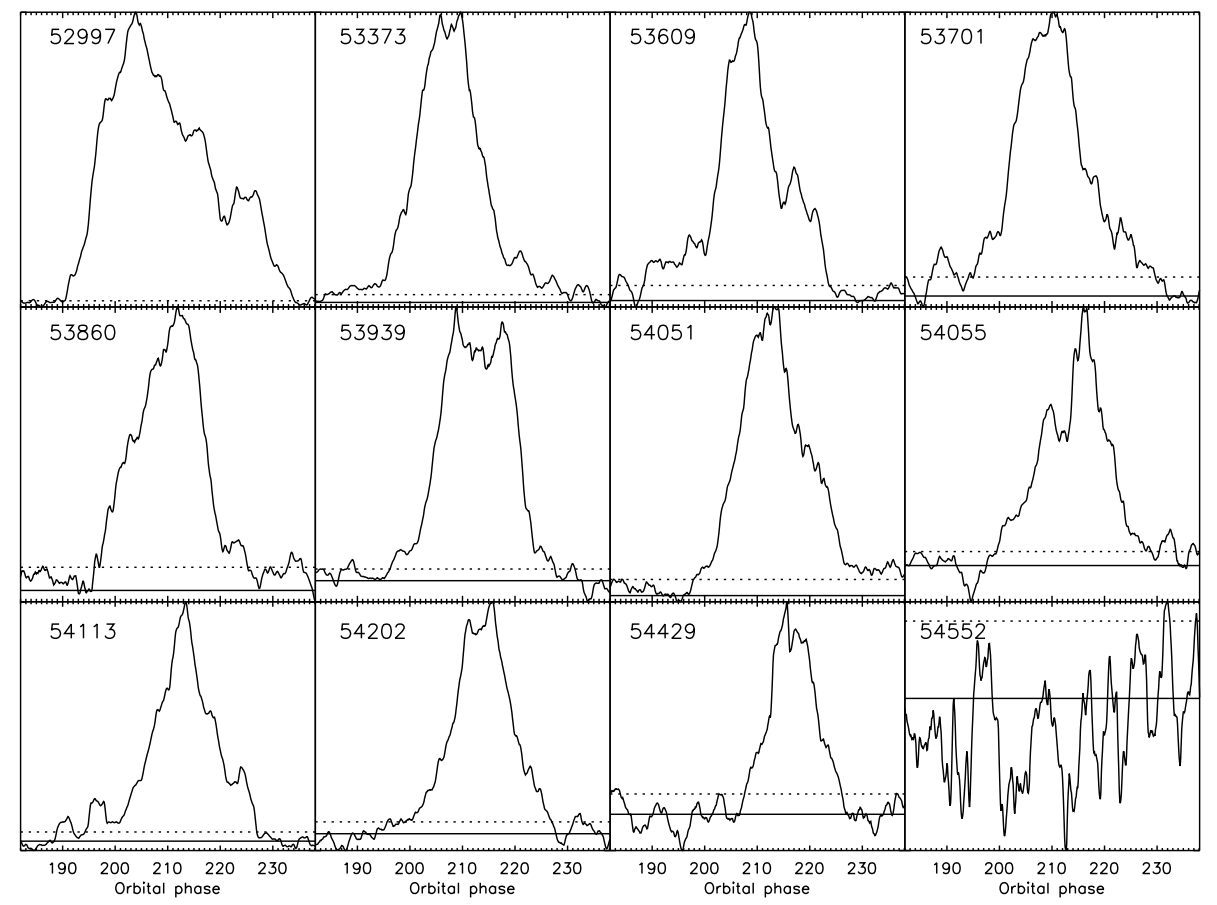

Figure 2.8: The light curves of BP1 on 12 different days at an observing frequency of $820 \mathrm{MHz}$. Each data set has been cleaned to get rid of radio frequency interference. All light curves have been smoothed by using a boxcar averaging technique with a width of 30 pulses. The horizontal solid and dotted lines show the baseline of the plot and the corresponding standard deviation of the off-peak region $\left(140^{\circ}-180^{\circ}\right.$ and $240^{\circ}-260^{\circ}$ ), respectively. The last data set shows just noise. 


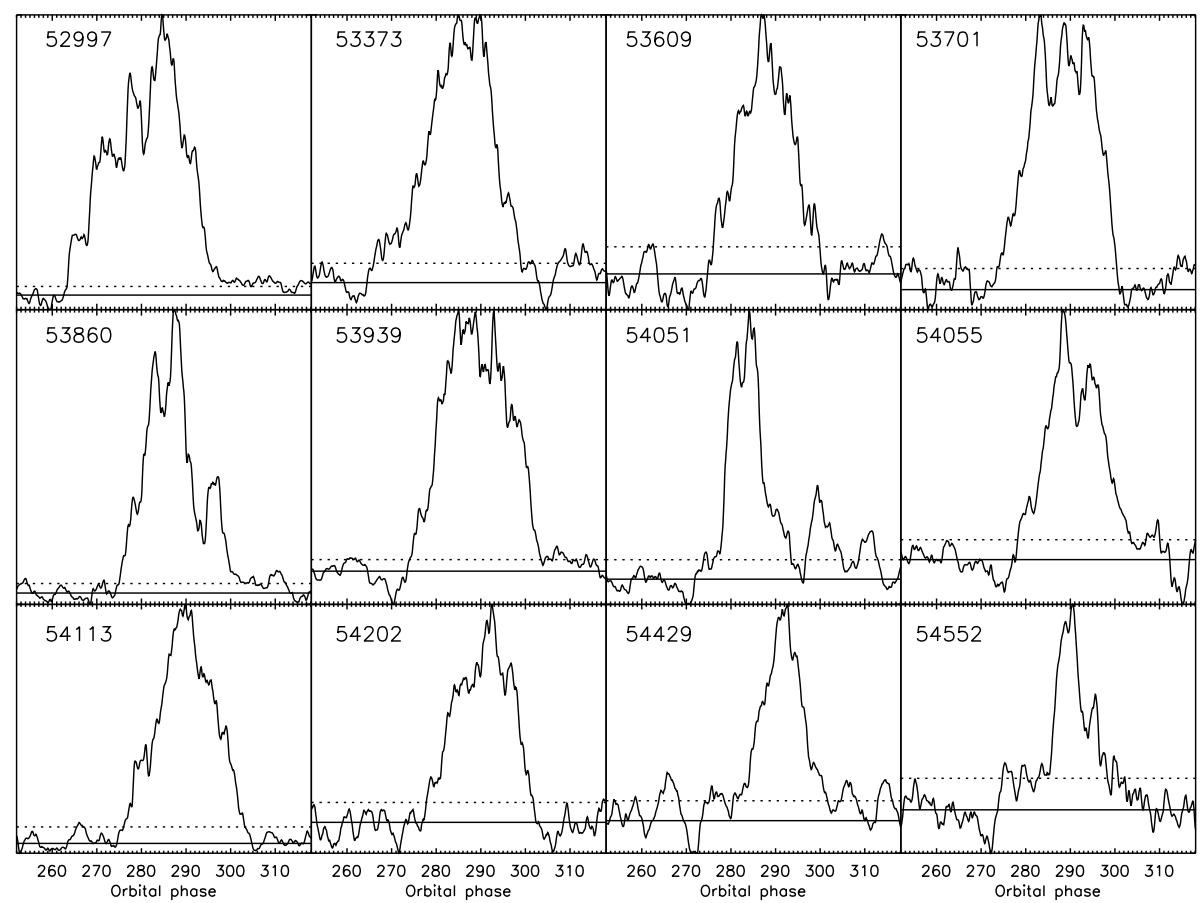

Figure 2.9: As in Figure 2.8, but for BP2.

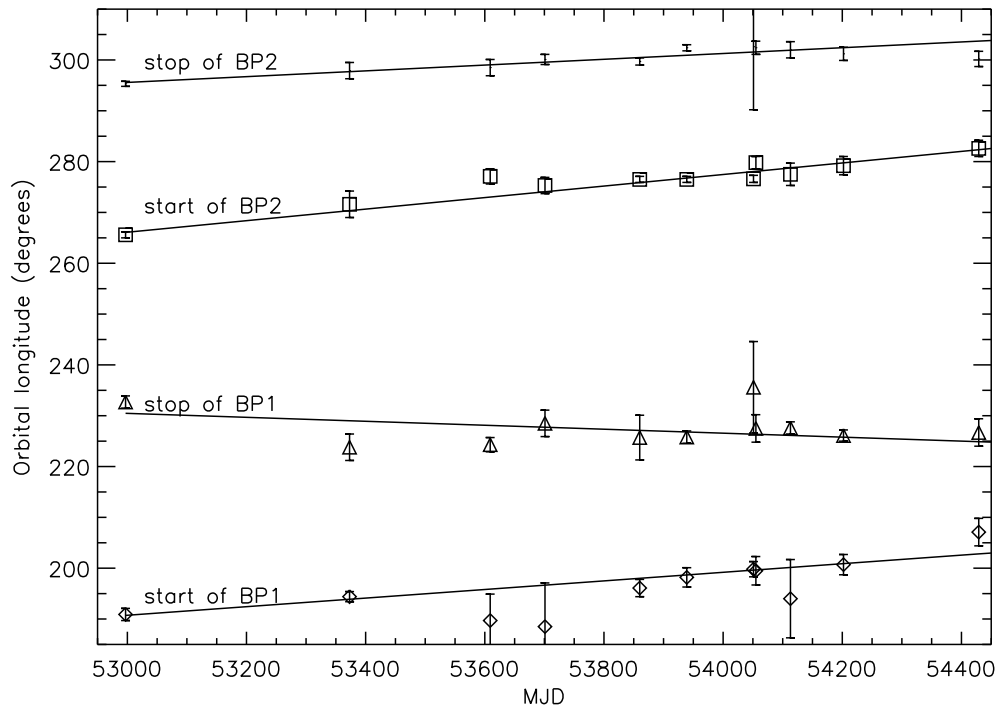

Figure 2.10: Evolution of the start and end orbital longitudes of BP1 and BP2, measured at the $10 \%$ of the maximum. 


\subsubsection{Analysis of the two weak phases}

Weak radio emission has been detected in two orbital phase ranges in addition to the two bright phases (see Table 1.2). One of the two weak emission regions, $340^{\circ}-30^{\circ}$ (hereafter WP1) is much brighter than the other, $80^{\circ}-130^{\circ}$ (hereafter WP2), and is detectable until 2007 November (MJD 54429) (see Figure 2.11). WP2 vanished in 2006 February (MJD 53783), more than a year before the disappearance of the WP1 (see Figure 2.12). In general, both weak phases show a unimodal pulse profile and no dramatic pulse shape changes over time. Some data show two apparent peaks in their pulse profile, but the lower signal-to-noise makes it difficult to model these in the same way as for the bright phases (e.g. on MJD 53702 in WP1 and 53701 in WP2). If the evolution is solely due to relativistic spin precession, we would expect the same profile evolution for all phases. However, the emission may not be strong enough in the weak phases to detect the second profile component. For instance, at MJD 54113 the brightness of the second brightest component of BP1 is $30 \%$ of the brightest component and that of BP2 is $67 \%$. In comparison, the rootmean-square noise in WP1 is almost 50\% of the pulse maximum, making it difficult to detect the second peak of the profile. Therefore, within the uncertainties, the component separation evolution may be similar for bright and weak phase profiles.

We then calculated the mean flux densities of both weak phase regions (see Figure 2.13). In these phases, the mean flux densities are lower than that of bright phases. The decreasing rates of the mean flux densities are calculated to be $0.032(8)$ and $0.02(2) \mathrm{mJy}_{\mathrm{yr}}{ }^{-1}$ for WP1 and WP2, respectively. The larger uncertainty of 


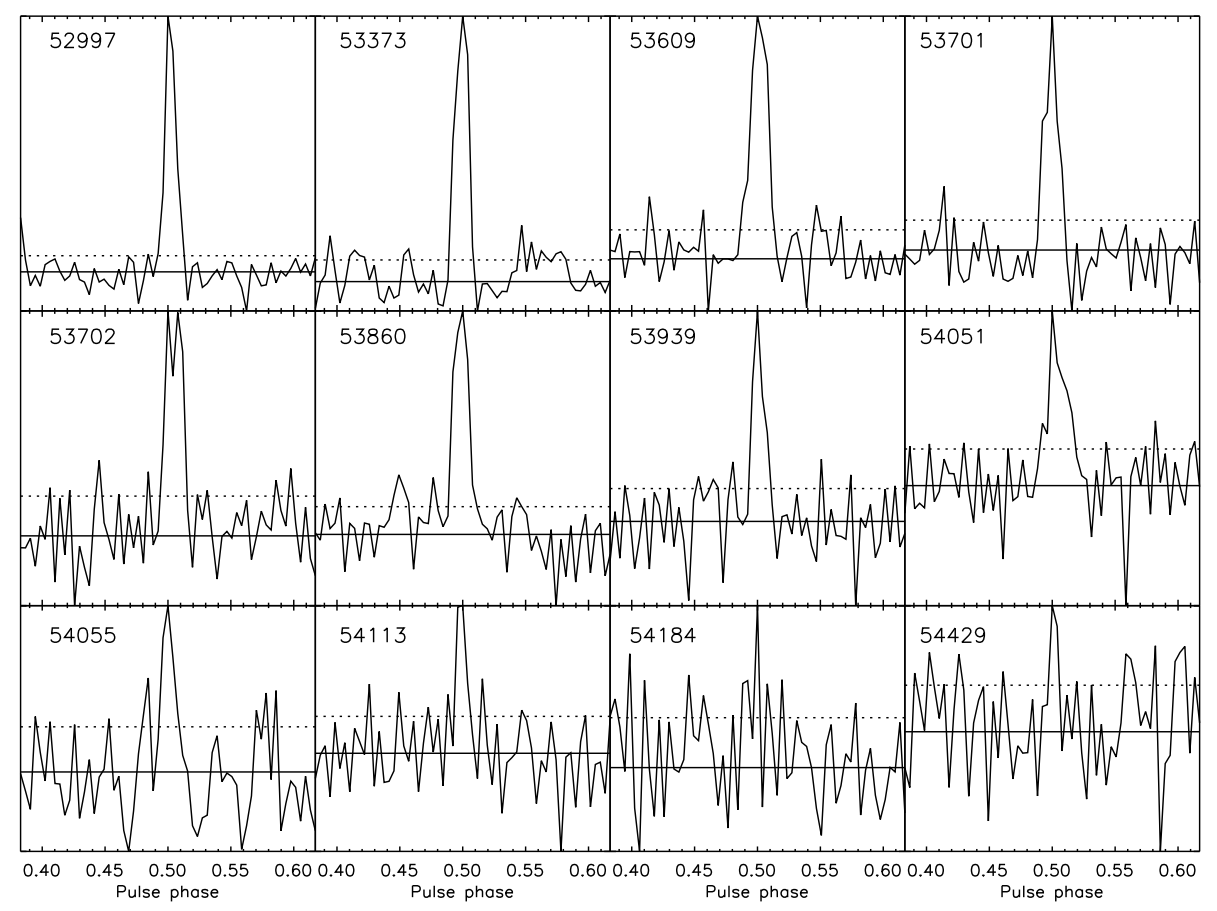

Figure 2.11: As in Figure 2.1, but for WP1. Each profile covers 20 min of orbital longitude (from $340^{\circ}-30^{\circ}$ ) and there are 256 bins across the entire profile.

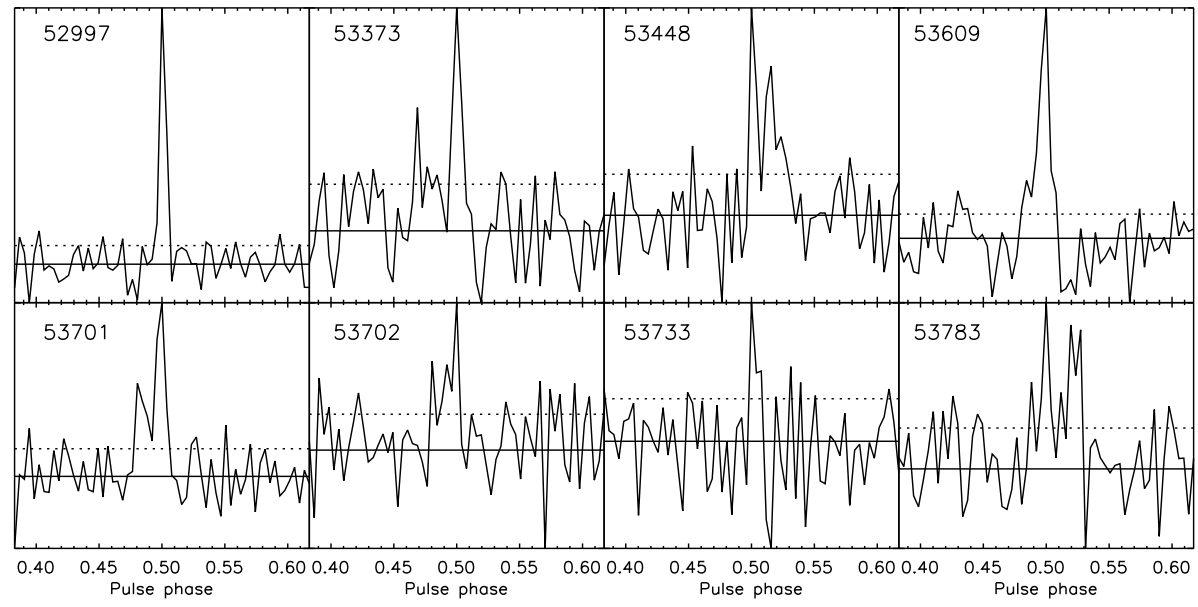

Figure 2.12: As in Figure 2.1, but for WP2 covering 20 min of orbital longitude (from $80^{\circ}-130^{\circ}$ ). The pulsar is weaker during this phase than during WP1. 

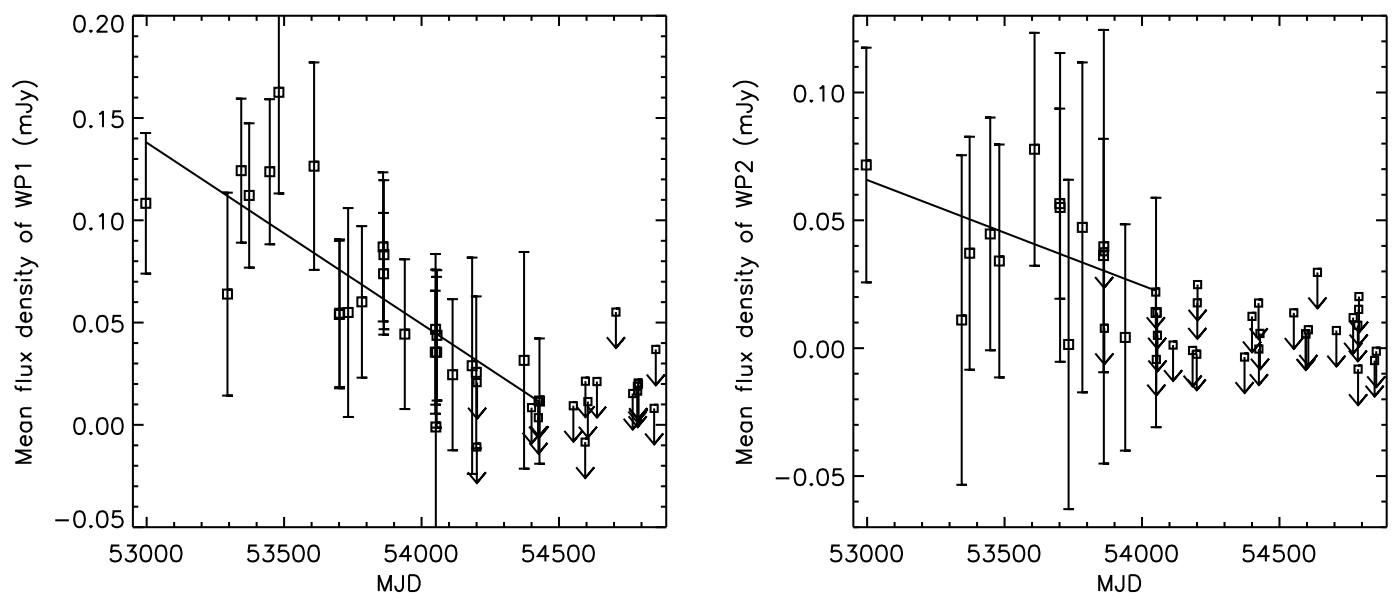

Figure 2.13: Mean flux density of the radio emission in WP1 (left) and in WP2 (right). The data marked by the arrows are the upper limits and ignored in our linear least-squares fits. The decreasing rate of the mean flux density is calculated to be $0.032(8)$ and $0.02(2) \mathrm{mJy} \mathrm{yr}^{-1}$ for WP1 and WP2, respectively.

the data points results in the poor quality of the fits. However, due to the weakness of the pulse emission in WP1 and WP2, we only use the two bright phase regions for our modeling.

\subsection{Determining the geometry of pulsar B with geodetic spin preces-} sion

Clifton \& Weisberg (2008) - hereafter CW08 - proposed a framework to model the 2-D pulse profile of a precessing pulsar by using a simple circular hollow-cone beam. They applied this model for PSR B1913+16 to explain its long term pulse profile evolution. To represent the different intensity levels of the emission beam, they constructed a set of coaxial circular hollow cones with different angular radii. Each circular cone represents a different intensity level. The outermost cone represents the lowest intensity level. The intensities gradually increase towards the 
maximum-intensity-level cone somewhere between the outermost cone and the center of the beam, and then decrease until reaching the center of the beam. With this special construction of the beam, it is possible to explain the hour-glass or oval shapes of the 2-D pulse profiles.

Moreover, this model constrains the geometrical parameters of the precessing

pulsar. Mainly, the model predicts the longitudinal separation (i.e., pulse width) at a given epoch for a given intensity level for some set of geometrical parameters. Then we fit this model-predicted width to the corresponding observed-pulse-profile width for a range of intensity levels to obtain the geometrical parameters.

First we used the circular hollow-cone beam shape in order to determine the geometry of the B pulsar. Then we used a more complicated elliptical horse-shoe shaped beam, filled with different intensity levels as in the circular beam construction, to constrain the geometry and found that this fit our observations better.

In both cases, the required pulse-width data at different intensity levels of the pulse profiles have been calculated by fitting Gaussians to the profiles and then calculating the pulse widths at different intensity levels of the fitted Gaussian curves. The modeling has been done for the two bright phases separately to constrain two sets of geometrical parameters.

\subsubsection{Modeling the geometry with a circular hollow-cone beam}

In this section, we use a circular hollow-cone beam and the same set of equations as in CW08 to determine the two angles $\alpha$ and $\theta$, which are the magnetic 
inclination (i.e. angle between spin axis and magnetic dipole moment) and the colatitude of the spin axis (i.e. angle between spin axis and orbital angular momentum), respectively (see Figure 2.14 (a)). The model also includes another parameter, $\mathrm{T}_{0}$, the epoch for which the precession phase is equal to zero.

The fit of the width at different intensity levels of our data to the model is shown in Figure 2.15. The fit has been done by minimizing the chi-square statistic over the entire parameter space of the two angles $(\alpha:[0, \pi], \theta:[0, \pi])$ and $\mathrm{T}_{0}:[1930,2001]$. This range of dates encompasses one full precessional cycle for pulsar B. The required errors of the data points for the chi-square statistic are obtained by the fitted Gaussians, which are the same for a given epoch at different intensity levels.

The best-fit results for the two angles for BP1 are $\alpha=80.0_{-15.0^{\circ}}^{\circ}$ and $\theta=$ $20.0_{-16.5^{\circ}}^{\circ}$, while for BP2 $\alpha=78.0^{\circ}+11.5^{\circ}$ and $\theta=20.0_{-12.5^{\circ}}^{\circ}$. A secondary peak in the PDFs caused large errors for the best fit parameters at their $68.5 \%$ confidence interval. The fits estimate $\mathrm{T}_{0}=1959.4(1) \mathrm{yr}$ for both phases. These best fit values are very different from those in Breton et al. (2008), who determined $\alpha=70.9(4)^{\circ}$ and $\theta=130.0(4)^{\circ}$ (see Table 2.1). In addition to this, the simple circular beam shape predicts a disappearance in $\sim 2018$ instead of 2008. Due to the poor fit and the inability to explain the disappearance of the radio emission in 2008, we experimented with other possible beam shapes. 


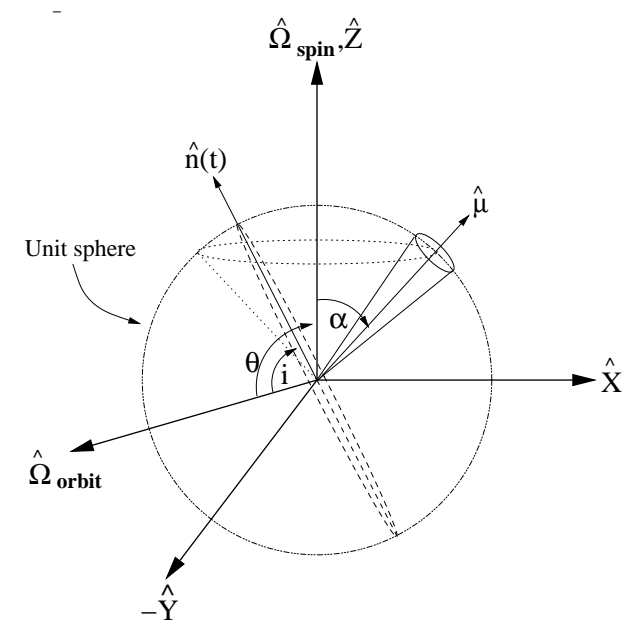

(a)

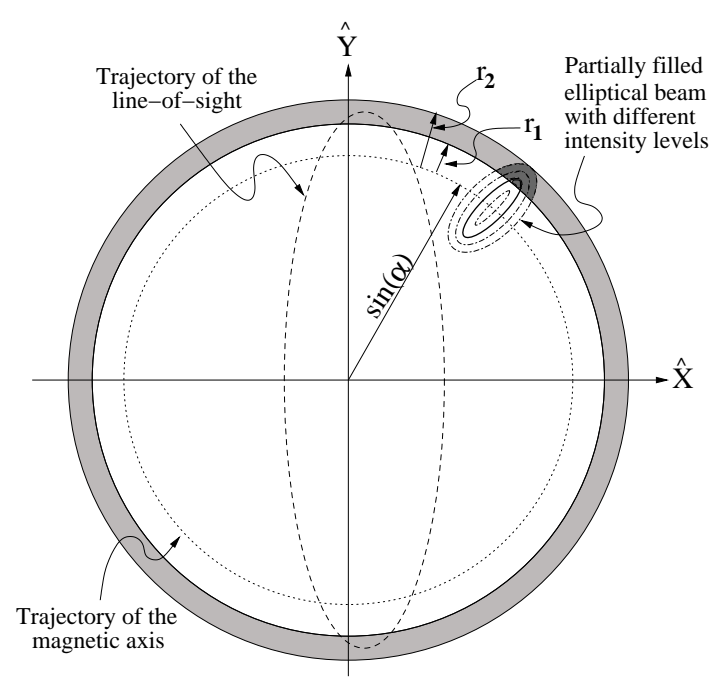

(b)

Figure 2.14: Geometry of pulsar B with its spin and the precession in a Cartesian coordinate system with a unit sphere at the center; all of the trajectories move on this sphere. (a) The coordinate system is chosen with the Z-axis parallel to the spin-axis of the pulsar $\left(\hat{\Omega}_{\text {spin }}\right)$ with the X-axis in the plane of the spin-axis and the orbital angular momentum axis $\left(\hat{\Omega}_{\text {orbit }}\right)$; i.e., the $\hat{\Omega}_{\text {spin }}$ and the $\hat{\Omega}_{\text {orbit }}$ are always in the X-Z plane. The pulsar-earth line-of-sight is $\hat{n}(t)$ and dashes represent the conical trajectory of it on the unit sphere about $\hat{\Omega}_{\text {orbit }}$ due to geodetic precession. Dots represent the conical trajectory of the co-rotating magnetic field $(\hat{\mu})$ on the unit sphere about $\hat{\Omega}_{\text {spin }}$ due to the pulsar spin. The inclination of the orbit is $i$. The colatitude of spin axis and the misalignment of the magnetic field are $\theta$ and $\alpha$, respectively. (b) The projections of trajectories of $\hat{\mu}$ and $\hat{n}(t)$ on the X-Y plane. Dash-dot lines represent the different intensity levels of the elliptical beam. The intensity increases gradually from the outer most edge of the beam to inwards until the maximum intensity level (the solid line of the beam), and then decreases towards the center of the beam. The lightly shaded region represents the detectable beam area when the beam sweeps about the spin axis. This area is determined from the radial lengths $r_{1}$ and $r_{2}$, and they are measured from the center of the beam on the $\mathrm{X}-\mathrm{Y}$ plane. With this restriction, the effective beam has a horse-shoe shape and it is represented as the dark shaded region. Dashes show the trajectory of the $\hat{n}(t)$ on the X-Y plane. When $\hat{n}(t)$ crosses the beam area (lightly shaded area), we are able to detect the pulsar. 

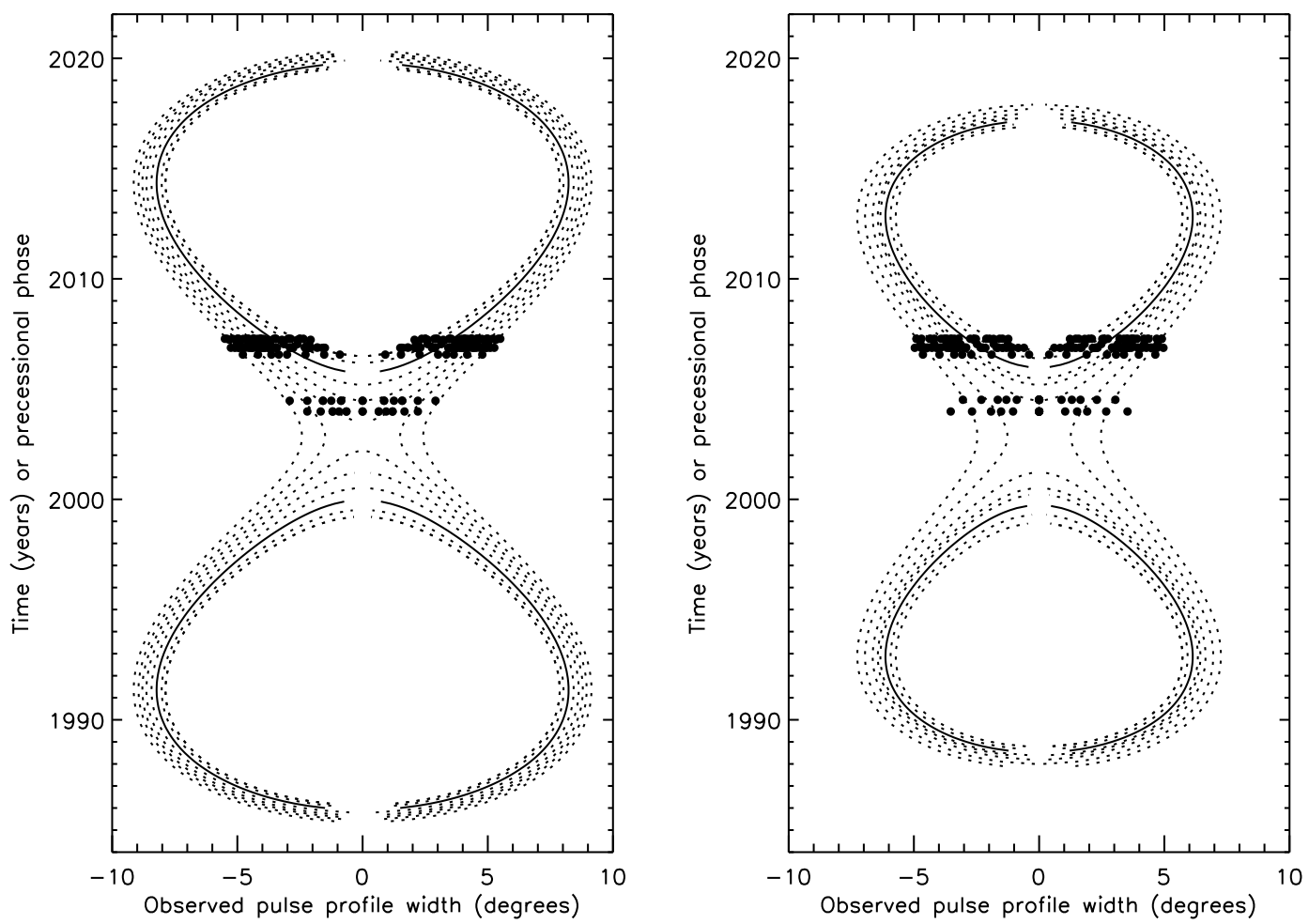

Figure 2.15: The hour-glass shaped two-dimensional pulse profile of PSR J0737-3039B, assuming a circular hollow-cone beam. The best fit parameters for BP1 are $\alpha=80.0^{\circ}+1.5^{\circ}$ and $\theta=20.0^{\circ}{ }_{-16.5^{\circ}}$ (left), and for BP2 are $\alpha=78.0^{\circ}+11.5^{\circ} 0^{\circ}$ and $\theta=20.0_{-12.5^{\circ}}^{\circ}$ (right). Fitting has been done by searching the entire range of the two angles. The dots are the widths at equal intensity of the pulse profile. Each horizontal row of dots represents an observation at a given epoch. Equal-intensity contours are produced from the emission of the circular symmetric conical beam. The solid lines are the equal intensity level of the peaks of two-peak pulse profile. The intensity increases from the inner dashed line outwards until the first solid line, which is the intensity of the peak, and then decreases outwards again. The intensity levels are, from inner dashed line, 80\%, 90\%, 100\%, 90\%, 80\%, 70\%, 60\% and $50 \%$. The vertical axis is calibrated in years, and can also be considered the spin precession phase, which increases linearly with time. The time of precessional phase zero in the fits is $\mathrm{T}_{0}=1959.4(1) \mathrm{yr}$ for both phases. We have omitted the data between MJDs 53175 and 53939. During this time, the profile was bi-modal but the separation between the peaks was small, making it difficult to measure widths at different intensity levels. 
Table 2.1: Geometrical parameters of pulsar B derived from our circular beam model (CBM), 2D elliptical beam model (2EBM), the eclipse model fitting by Breton et al. (2008), and the 3D elliptical beam model (3EBM). Note that 3EBM is the reference model for pulsar B and we use the corresponding geometry in the emission altitude analysis in Chapter 3.

\begin{tabular}{llc}
\hline & \multicolumn{1}{c}{$\alpha\left(^{\circ}\right)$} & $\theta\left(^{\circ}\right)$ \\
\hline CBM :BP1 & $80.0(-15.0,+1.5)$ & $20.0(-16.5,+1.5)$ \\
CBM :BP2 & $78.0(-11.0,+1.5)$ & $20.0(-12.5,+1.0)$ \\
2EBM :BP1 & $65.0(-1.0,+2.0)$ & $134.5(-6.0,+1.5)$ \\
2EBM :BP2 & $73.0(-3.5,+1.4)$ & $138.5(-7.5,+4.0)$ \\
Breton et al. $(2008)$ & $70.9(-0.4,+0.4)$ & $130.0(-0.4,+0.4)$ \\
\hline 3EBM & $61.0(-2.4,+7.9)$ & $138.5(-4.4,+5.3)$ \\
\hline
\end{tabular}

\subsubsection{Modeling the geometry with a 2-dimensional elliptical horse- shoe shaped beam}

In this case, we used an elliptical hollow-cone filled beam with different intensity levels and then assumed that only a part of this beam is detectable, resulting in a horse-shoe shaped beam. To simplify the geometry, we assumed that the beam shape is fixed with respect to the neutron star and it rotates around the spin axis in a way such that the semi-major axis is always aligned in the radially outward direction from the spin axis (see Figure 2.16).

In order to model this particular shape, we derived an equation for the profile width

$$
w_{j}(t)=\arcsin \left[2 \sqrt{\left[n_{x}(t)\right]^{2}+\left[n_{y}(t)\right]^{2}} \sin \eta_{j}(t)\right]
$$

where $w_{j}(t)$ is the width of the pulse profile at a given time, with the subscript $j$ 


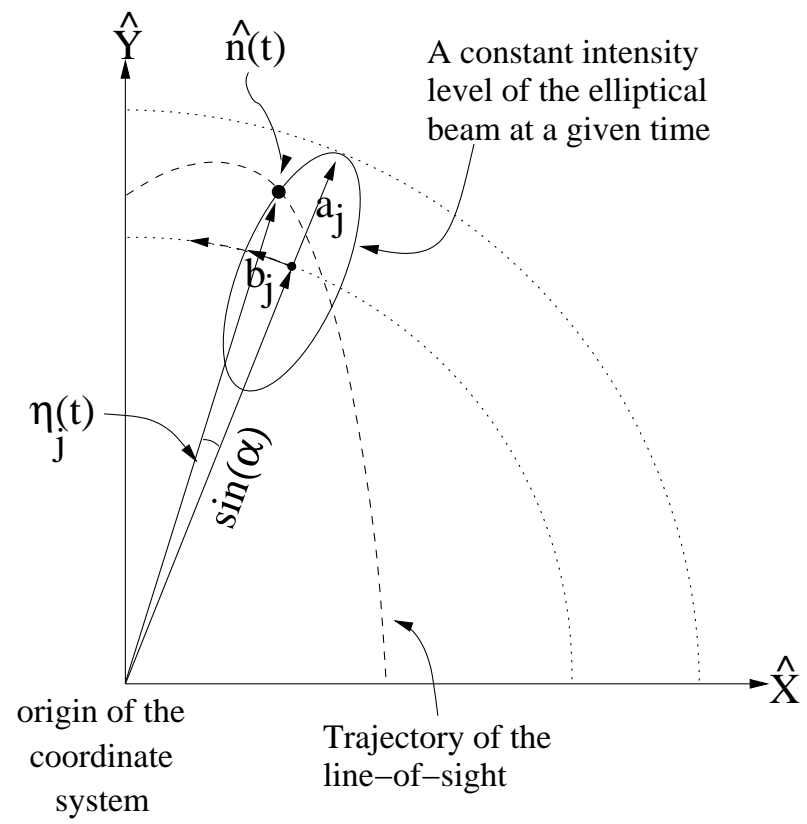

Figure 2.16: A zoomed-in view of the elliptical beam on the X-Y plane with a constant intensity level. The given constant intensity level crosses our line-of-sight when the line-of-sight is within the effective beam area (lightly shaded area in Figure 2.14(b)). The semi-major and semi-minor axes, $a_{j}$ and $b_{j}$, can take any value corresponding to the intensity level in a way such that the ratio $\mathrm{a}_{j} / \mathrm{b}_{j}$ is a constant. After projecting to the $\mathrm{X}-\mathrm{Y}$ plane, the semi-major axis is aligned in the radially outward direction from the spin axis. $\sin (\alpha)$ is the distance to the center of the beam from the spin axis on the X-Y plane. $\eta_{j}(t)$ is defined also on the X-Y plane, and it is the angle between the line joining the origin with the point at which the line-of-sight $(\hat{n}(t))$ encounters the constant intensity level of the beam and the line joining the spin axis with the center of the elliptical beam. 
specifying the intensity level of the profile (please see Appendix A for a detailed derivation). The line-of-sight vector, $\hat{n}(t)$, can be expressed as in equation (3) of CW08

$$
\hat{n}(t)=\left(\begin{array}{c}
n_{x}(t) \\
n_{y}(t) \\
n_{z}(t)
\end{array}\right)=\left(\begin{array}{c}
-\cos \theta \sin i \cos \phi_{\text {prec }}(t)+\sin \theta \cos i \\
\sin i \sin \phi_{\text {prec }}(t) \\
-\sin \theta \sin i \cos \phi_{\text {prec }}(t)-\cos \theta \cos i
\end{array}\right)
$$

and $n_{x}(t)$ and $n_{y}(t)$ in the above equation are the $\mathrm{X}$ and $\mathrm{Y}$ components of $\hat{n}(t)$. The third variable, $\eta_{j}(t)$, is the angle which is subtended at the origin of the coordinate system due to the encounter of the line-of-sight vector with the elliptical beam; namely, it is the angle between the line joining the origin with the point at which the line-of-sight vector encounters the beam and the line joining the origin with the center of the elliptical beam (see Figure 2.16). Thus, amending the expression of CW08 for an elliptical beam, $\eta_{j}(t)$ can be expressed as

$$
\eta_{j}(t)=\operatorname{acos}\left[\frac{n^{2}(t)+\sin ^{2} \alpha-[n(t)-\sin \alpha]^{2}\left[1-R_{j}^{2}\right]+b_{j}^{2}}{2 n(t) \sin \alpha}\right]
$$

where $n(t)=\sqrt{n_{x}(t)^{2}+n_{y}(t)^{2}}, R_{j}=b_{j} / a_{j}$ and $a_{j}$ and $b_{j}$ are the semi-major and semi-minor axes of the elliptical beam for a given intensity level (see Appendix A for the derivation). They can take different values corresponding to the intensity levels of the beam in a way such that $a_{j} / b_{j}$, or the ellipticity of the beam, is a constant.

The horse-shoe shaped beam is a subsection of the elliptical beam. We can construct this by restricting the detectable area of the beam to a section of the 
elliptical beam, with $r_{1}$ and $r_{2}$ the radial distances on the $\mathrm{X}-\mathrm{Y}$ plane of the elliptical beam within which emission is detectable (see Figure 2.14 (b)). Simply, we can detect the emission beam of the pulsar only when $\sin \alpha+r_{1}<\sqrt{\left[n_{x}(t)\right]^{2}+\left[n_{y}(t)\right]^{2}}<$ $\sin \alpha+r_{2}$, where $n_{x}(t)$ and $n_{y}(t)$ are the $\mathrm{X}$ and $\mathrm{Y}$ components of $\hat{n}(t)$.

The fit has been done for the two bright phases separately by searching over the entire parameter space for the two angles $\alpha$ and $\theta$ and the ratio $a / b$ using a likelihood analysis (see, e.g., McLaughlin \& Cordes, 2000). First the individual likelihood of each data point was calculated by assuming a Gaussian distribution such that the likelihood for model $\Theta$ and measurement $i$ is $\mathcal{L}_{i}(\Theta)=\left(2 \pi \sigma_{i}^{2}\right)^{-1 / 2} \exp \left(-\left(w_{\text {model }, i}-\right.\right.$ $\left.\left.w_{\text {measured }, i}\right)^{2} / 2 \sigma_{i}^{2}\right)$, where $w_{\text {model }, i}$ and $w_{\text {measured }, i}$ are the model and measured widths and $\sigma_{i}$ is the error on the measured width. We then multiplied all $\mathcal{L}_{i}(\Theta)$ for all data points $i$ (i.e. profiles from all epochs and at all intensity levels) to get the total likelihood $\mathcal{L}(\Theta)$ for parameter combination $\Theta$. $\mathcal{L}_{\text {tot }}$, the total likelihood over all parameter combinations, was calculated by summing all the likelihoods over all parameter combinations. Then the posterior probability distributions for parameter values were calculated by dividing the likelihoods for a particular parameter value by the total likelihood $\mathcal{L}_{\text {tot }}$, assuming flat prior probability distributions. The posterior probabilities of the three parameters $\alpha, \theta$, and a/b for BP1 are shown in Figure 2.17 and the best fit 2-D pulse profile is shown in Figure 2.18.

The fitting constrained the two angles for BP1 to be $\alpha=65.0^{\circ}+2.0^{\circ}$ and $\theta$ $=134.5_{-6.0^{\circ}}^{\circ}$, while for BP2 $\alpha=73.0_{-3.5^{\circ}}^{\circ}$ and $\theta=138.5_{-7.5^{\circ}}^{\circ}$. We expect the same estimates for the two angles in both bright phases, indicating that our errors are underestimated or the model may not fit the data perfectly. These angles are 

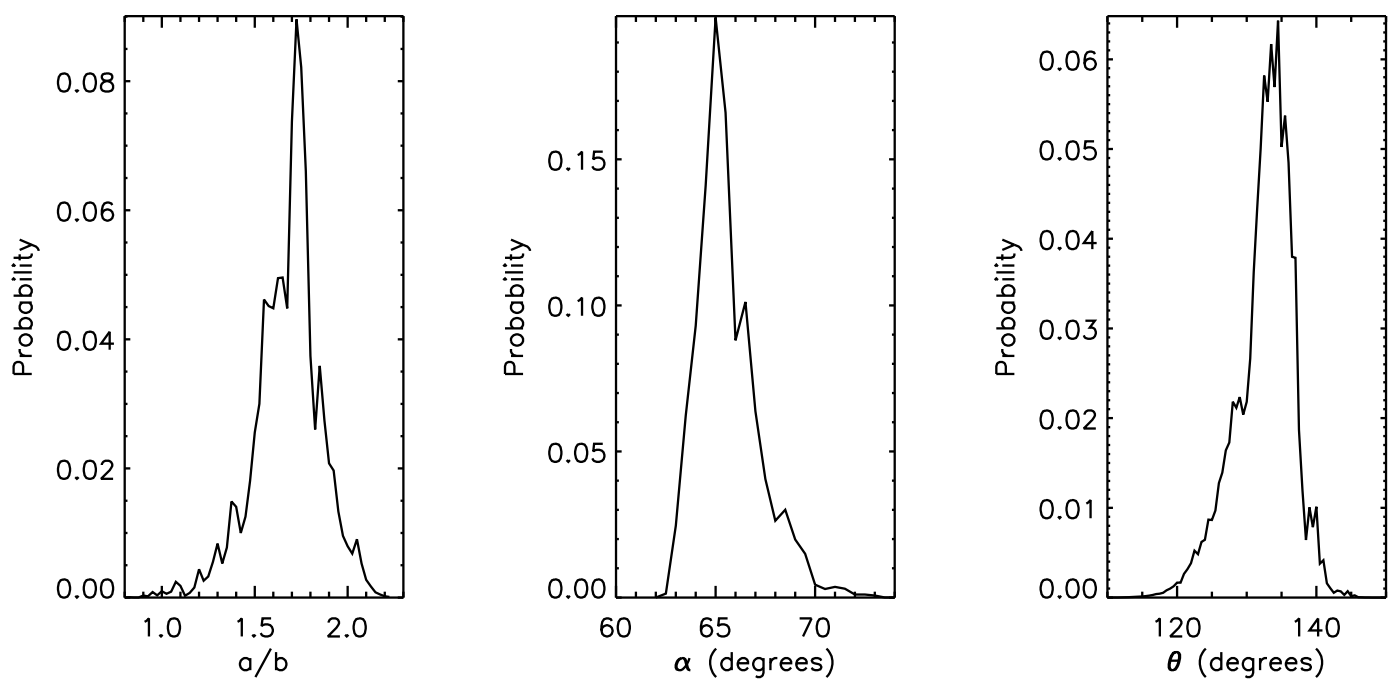

Figure 2.17: The calculated posterior probabilities of the parameter values given by likelihood analysis for BP1. The best fit parameters and their $68 \%$ confidence intervals are estimated to be $\mathrm{a} / \mathrm{b}=1.72_{-0.20}^{+0.12}, \alpha=65.0^{\circ}{ }_{-1.0^{\circ}}$ and $\theta=134.5^{\circ}+1.5_{-6.0^{\circ}}^{\circ}$.

similar to those derived by Breton et al. (2008) (see Table 2.1). The best-fit estimate of the ratio of the beam semi-major and semi-minor axes $(a / b)$ is $1.72_{-0.20}^{+0.12}$ and $1.15_{-0.22}^{+0.27}$ for $\mathrm{BP} 1$ and $\mathrm{BP} 2$, respectively. Because of the varying influence of $\mathrm{A}$, the magnetosphere could have different shapes at different orbital phases, and these angles could in fact differ. In order to cause the disappearance in 2008, the ratio $r_{1} / r_{2}$ has to be 0.70 and 0.48 for $\mathrm{BP} 1$ and $\mathrm{BP} 2$, respectively. The parameter $\mathrm{T}_{0}$ is $1950.3(1)$ yr for both bright phases. Since the spin axis takes $71 \mathrm{yr}$ to precess a full cycle around the orbital angular momentum axis, the year 2008, within which disappearance occurred, is not a special year relative to this estimated $T_{0}$.

After transforming the ratio $a / b$ to the assumed unit sphere by dividing $\cos (\alpha)$, we estimate the ellipticity of the radio beam of B in the two bright phases separately: $0.9(1)$ and $0.9(2)$ at $\mathrm{BP} 1$ and $\mathrm{BP} 2$, respectively. The ratio $r_{1} / r_{2}$ in the two bright phases reveals that only $(29 \pm 6) \%$ of the beam area was detectable in BP1 and 

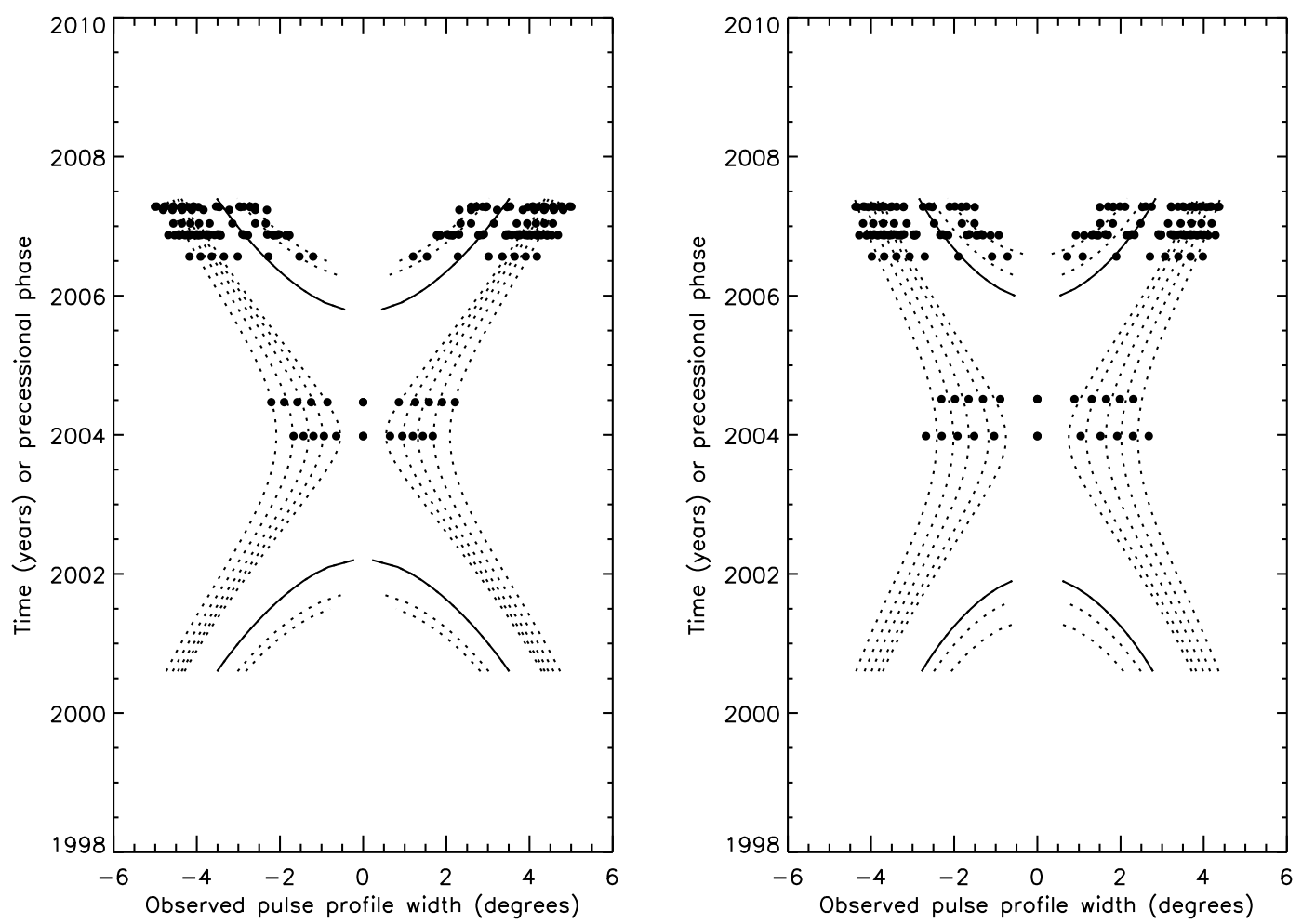

Figure 2.18: The two-dimensional pulse profile of PSR J0737-3039B, assuming a horse-shoe shaped beam. The best fit parameters for BP1 are $\alpha=65.0_{-1.0^{\circ}}^{+2.0^{\circ}}, \theta=$ $134.5_{-6.0^{\circ}}^{\circ}$, and $\mathrm{a} / \mathrm{b}=1.72_{-0.20}^{+0.12}$ (left). For BP2, the best fit parameters are $\alpha=$ $73.0^{\circ}{ }_{-3.5^{\circ}}, \theta=138.5^{\circ}+4.0^{\circ}$, and $\mathrm{a} / \mathrm{b}=1.15_{-0.22}^{+0.27}$ (right). We have omitted the data between MJDs 53175 and 53939. During this time, the profile was bi-modal but the separation between the peaks was small, making it difficult to measure widths at different intensity levels. 
only $(21 \pm 5) \%$ in BP2. The different estimates for the effective area of the beam in the two bright phases show that the emission beam may have different orientations corresponding to the different orbital phases. The characteristic pulse shapes are also different at different orbital phases. This is a direct consequence of the influence of pulsar A's wind on the emission beam of pulsar B (see, e.g., Lyutikov (2004)).

The data show that the pulse profile had two peaks when the disappearance occurred in 2008 and clearly did not become a single peak before the disappearance. The horse-shoe shaped beam model can explain this phenomenon qualitatively, as the line-of-sight moved out of the elongated side of the beam (i.e. the two opened arms of the horse-shoe beam, see Figure $2.14(\mathrm{~b})$ ). This results in the disappearance of both peaks of the pulse profiles in both bright phases simultaneously. The reappearance of the emission of pulsar B is predicted to occur in $\sim 2035$, according to the horse-shoe beam model, assuming emission from the same part of the beam. This is because our line-of-sight encounters the emission beam twice during the time that the spin axis rotates around the orbital angular momentum axis once $(\sim 71 \mathrm{yr})$. This can be understood by visualizing the geometry of the emission beam with two angles, $\alpha \approx 65^{\circ}$ and $\theta \approx 130^{\circ}$. Thus the spin axis of the pulsar $\mathrm{B}$ is below the inclination plane and the magnetic moment axis crosses the inclination plane twice during every $2.8 \mathrm{sec}$ period. Since the inclination plane of the system is almost edge-on with respect to the Earth, we are able to detect the emission beam twice during the 71 yr period. Geodetic precession along with the unique geometry of the system is responsible for this phenomenon. Note that this prediction for the reappearance time is only valid assuming a single horse-shoe emission component. If the 
beam is symmetric, with two horse-shoe shaped emission components separated by a gap, then we would expect a reappearance around 2014. Because we have only sampled a limited part of the beam, it is impossible to know what the true intensity distribution of B's beam is at this point.

\subsubsection{Revisiting the geometry with a 3-dimensional elliptical beam model}

For the emission height calculation which we discuss in Chapter 3, the angular radius of the emission beam is required. In Section 2.3.2, we claimed the beam shape of pulsar B is elliptical by modifying the CW08 geometrical framework. In this model, we used the $2 \mathrm{D}$ geometry of the beam after projecting it to a plane which is perpendicular to the spin axis, resulting in a projected angular radius. We improve this model by using a more realistic 3D model in this section to determine the actual angular radius of the beam.

We use the same data set reported in Section 2.2. However, we use better time resolution pulse profiles in this analysis compared to the previous one given in Section 2.2.1. The mean pulse profiles for BP1 are shown in Figure 2.19. The second peak of the pulse profile can be hidden with the low time resolution. Therefore, we use 1024 bins across the full pulse phase, resulting in an effective time resolution of $0.003 \mathrm{~s}$ in this model compared to $0.01 \mathrm{~s}$ in the previous analysis. For example, the second peak of the pulse profile of MJD 53860 around pulse phase 0.52 in Figure 2.19 cannot be clearly seen in Figure 2.1 on the same day with low time 


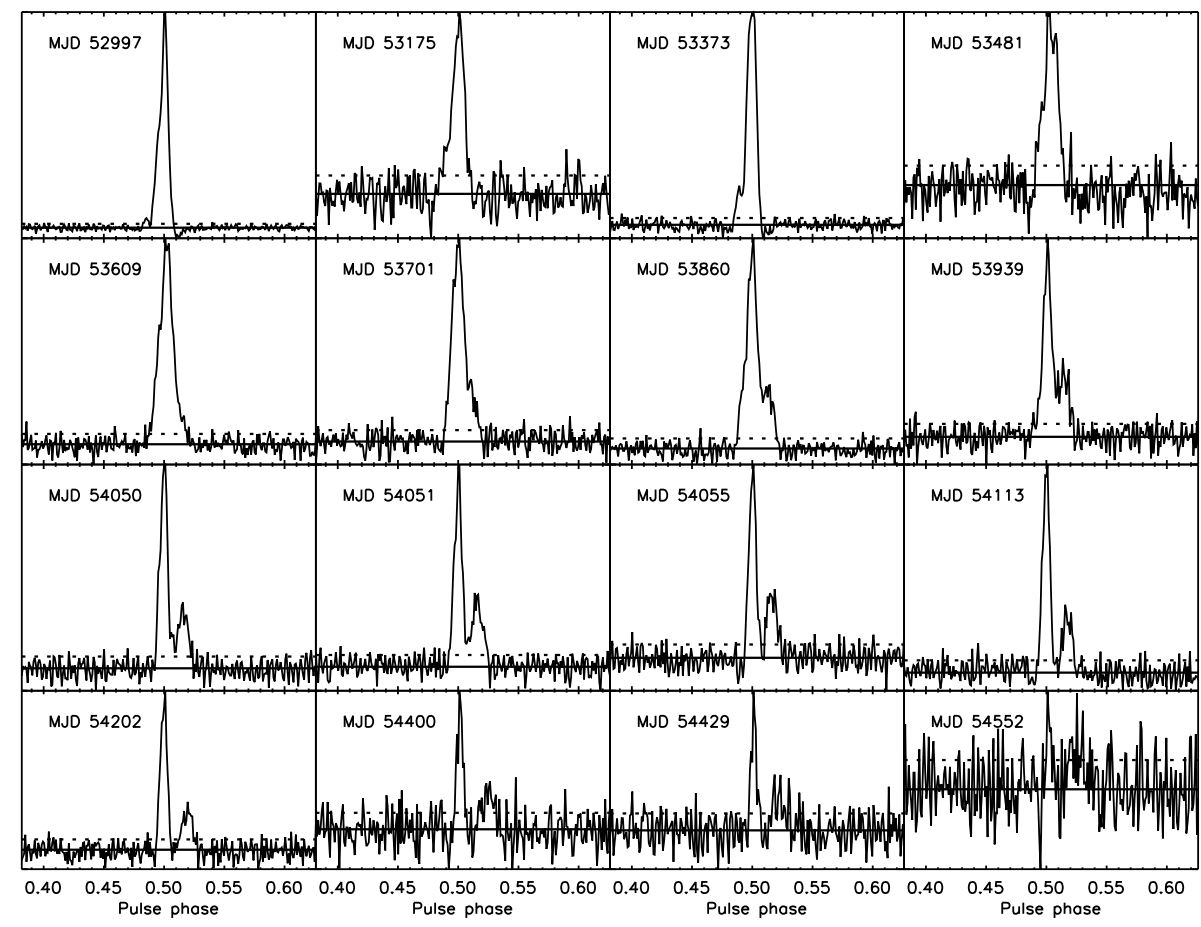

Figure 2.19: Mean pulse profiles for BP1 on 16 different days including the very first observation which was made on 2003 December 24 (MJD 52997). All data have been observed at a frequency of $820 \mathrm{MHz}$. There are 1024 bins across the entire pulse profile, resulting an effective time resolution of $3 \mathrm{~ms}$. Since predictions of absolute pulse phase are not available for these observations, we aligned the maximum peak to the pulse phase of 0.5 at each epoch. The horizontal and dotted lines show the baseline, or off-pulse mean, of the profile and the standard deviation of the off-peak region, respectively. The signal-to-noise ratio $(\mathrm{S} / \mathrm{N})$ of pulse profiles has decreased significantly from 284 (on MJD 52997) to 11 (on MJD 54552).

resolution. We use the pulse profiles from 23 days in this model, including 16 epochs in Figure 2.19, in order to derive the beam shape. We include some low signal-tonoise data (e.g. MJD 53481) because the second peak became apparent around those days. Because these profiles appeared as single-peaked, we ignored them in the geometrical modeling of the previous lower time resolution study. Similar to the previous section, we fit one and two Gaussians for each single and double-peaked pulse profile, respectively, and then calculate profile widths at different intensity levels. 
The angular radius of a circular emission beam has been calculated using pulse profile widths and an assumed emission geometry (Gil et al., 1984). Since the shape of B's beam is not circular, we attempt to derive an approximate equation for an elliptical beam in 3D. First, we construct the beam with a set of coaxial hollow cones, as in the previous section, representing different intensity levels in a way such that the outermost one represents the lowest intensity level and then the intensity increases gradually inwards toward the maximum and then decreases until reaching the center of the beam. All these cones have cross sections with a constant ellipticity of $a_{j} / b_{j}$, where $a_{j}$ and $b_{j}$ are semi-major and minor axes, respectively, of each hollow-cone beam. Then, for any given longitudinal angular radius $\rho_{l, j}(t)$ (see Figure 2.20), magnetic misalignment angle $\alpha$, and impact parameter $\beta(t)$, the pulse profile width $w_{j}(t)$ can be derived from spherical trigonometry (i.e. from spherical triangle FBD in Figure 2.20) as follows

$$
w_{j}(t)=2 \arccos \left(\frac{\cos \left(\rho_{l, j}(t)\right)-\cos ^{2}(\alpha+\beta(t))}{\sin ^{2}(\alpha+\beta(t))}\right),
$$

where subscript $j$ specifies different intensity levels of the pulse profile. Note that $\rho_{l, j}(t)$ is time dependent because the region where our line-of-sight cuts the beam is changing with time due to precession. The impact parameter is given in the form

$$
\begin{aligned}
& \cos \zeta(t)=\sin \theta \cos \phi_{\text {prec }}(t) \sin i+\cos \theta \cos i \\
& \beta(t)=\zeta(t)-\alpha .
\end{aligned}
$$


where $\zeta(t)$ is the angle between the spin axis and our line-of-sight at a given time and $\phi_{\text {prec }}(t)=\Omega_{\text {prec }}\left(t-T_{0}\right)$. In order to relate $\rho_{l, j}(t)$ with the elliptical beam shape, we derive an equation with the assumption that the cross-section of the beam is small enough to use 1D trigonometry (see Appendix B for a detailed derivation). Then the longitudinal angular radius $\rho_{l, j}(t)$ can be given as a function of $\beta(t)$,

$$
\rho_{l, j}(t)=\frac{1}{\chi} \sqrt{\sin ^{2}\left(\rho_{a, j}\right)-\cos ^{2}\left(\rho_{a, j}\right) \tan ^{2}(\beta(t))},
$$

where $\rho_{a, j}$ is the angular radius across the semi-major axis of the beam (see Figure 2.20) for a given intensity level and $\chi=a_{j} / b_{j}$, which is a constant for all different intensity cones. This expression shows that the minimum $\rho_{l, j}(t)$ of zero occurs when the line-of-sight just encounters the beam (i.e. $\beta(t)=\rho_{a, j}$ ), resulting in $w_{j}(t)=0$. The maximum $\rho_{l, j}(t)$ occurs when the line-of-sight crosses the center of the beam (i.e. $\beta(t)=0$ ), which leads to the maximum $w_{j}(t)$. Therefore, by combining equation (2.5) and (2.7) for a given $\alpha, \beta(t)$, and $\rho_{a, j}$, we can calculate the pulse profile width $w_{j}(t)$ for any given intensity level.

In order to determine the geometry of $\mathrm{B}$, we fit the model-predicted pulse profile widths to observed pulse profile widths of BP1 at different intensity levels using the same likelihood analysis that is described in Section 2.3.2. The fit was done by searching the entire parameter space of $\alpha, \theta, \chi$, and $T_{0}$. For each combination of these parameters, we vary $\rho_{a, j}$ from $0^{\circ}$ to $30^{\circ}$ freely until we reach the best solution. Then we use a maximum likelihood analysis to determine the best-fit geometrical parameters. The best-fit model for BP1 is shown in Figure 2.21. The 


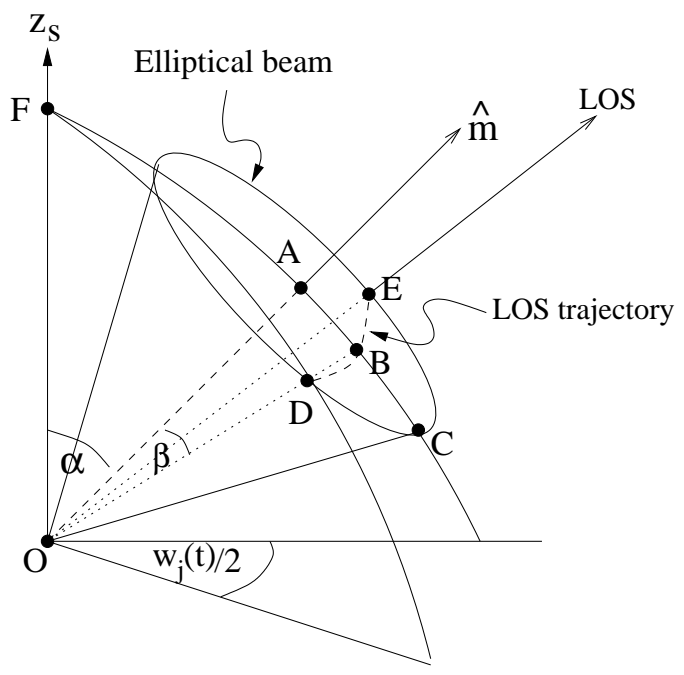

Figure 2.20: Elliptical emission cone in the frame $\left(x_{s}, y_{s}, z_{s}\right)$. The magnetic axis of the beam represents with $\hat{m}$ and it is misaligned with the spin axis of an angle $\alpha$. The trajectory of the line-of-sight across the beam due to rotation is denoted with $D B E$. The angle $B \hat{O} D$ is the longitudinal angular radius $\rho_{l, j}(t)$ of the beam for a given intensity level at a given time. The angle $A \hat{O} C$ is the angular radius across the semi-major axis of the beam $\rho_{a, j}$ for a given intensity level, which is time independent and fixed for the beam. The angle $A \hat{O} D$ is the effective angular radius of the beam $\rho_{e, j}(t)$ for a given intensity level.

estimated geometrical parameters $\alpha=61.0_{-2.4^{\circ}}^{\circ+7.9^{\circ}}$ and $\theta=138.5_{-4.4^{\circ}}^{\circ+5.3^{\circ}}$ are consistent with those derived in Section 2.3.2 and Breton et al. (2008) within the 2- $\sigma$ errors (see Table 2.1). The ratio $\chi$ is constrained to be $2.6_{-0.6}^{+0.4}$, lower than the estimate of the previous 2D model. Our new estimate is more believable because it has been derived from a full 3D viewing model. In addition to these parameters, we derive $T_{0}$ to be MJD $57399_{-25}^{+4}$ (2016 January 12), which results in a precessional phase of $46^{\circ}$ at an epoch of MJD 54050 (2006 November 11). This estimate is consistent with the value predicted by Breton et al. (2008) at the same epoch. However, this is a somewhat arbitrary parameter that can be chosen from our best-fit model. Note that the best-fit $T_{0}$ in 2D model is about MJD 33360 (1950 March 20), which results in a precessional phase of $73^{\circ}$ at an epoch of MJD 54050. These two best-fit $\phi_{\text {prec }}$ 


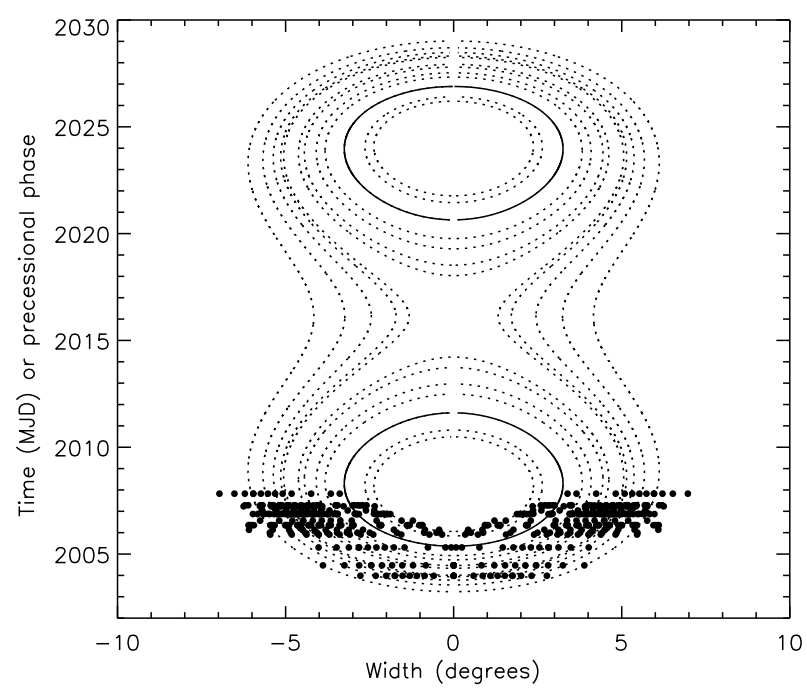

Figure 2.21: The 2D pulse profile of BP1, assuming the 3D elliptical hollowcone beam model. The best-fit geometrical parameters are $\alpha=61.0_{-2.4^{\circ}}^{+7.9^{\circ}}$, $\theta=138.5_{-4.4^{\circ}}^{+5.3^{\circ}}$ and $\chi=2.6_{-0.6}^{+0.4}$ (errors are $1 \sigma$ ). The corresponding $T_{0}$ is MJD 57399 (2006 November 11), which is the time where the spin axis of the pulsar is in the plane of our line-of-sight and the orbital angular momentum axis. Note that these $\alpha$ and $\theta$ values are consistent with the results of $2 \mathrm{D}$ elliptical beam model (Section 2.3.2) and (Breton et al., 2008). The intensity levels are, from the inner dashed line, $80 \%, 90 \%, 100 \%, 90 \%, 80 \%, 70 \%, 60 \%, 50 \%, 40 \%, 30 \%, 20 \%$ and $10 \%$. The vertical axis is calibrated in years and can also be considered the spin precession phase. Note that, this figure shows the emission from the full elliptical beam. If the beam is partially-filled according to 2008 radio disappearance, then the model predicts no radio pulse profiles from 2008 to 2024 .

result in a shift of the hour-glass $2 \mathrm{D}$ pulse profile shape along the precessional phase or time axis (see Figure 2.21 and Figure 2.18).

The angular radius across the semi-major axis of the beam at the maximum intensity level $\rho_{a, 100}$ and $10 \%$ of the maximum $\rho_{a, 10}$ are constrained to be $9.9^{\circ}$ and $14.3^{\circ}$, respectively. In order to determine the effective angular radius of the beam (more details are given in Section 3.4), we use these angular radii with the derived beam geometry.

For emission height estimates for normal non-precessing pulsars, a beam shape is not essential because our line-of-sight always observes the same section of the 
emission beam. However, for precessing pulsars, we must consider a beam shape in order to determine the emission height due to observing different sections of the emission beam. Therefore, in this particular case, we use our derived 3D elliptical beam shape with the best-fit geometrical parameters of $\mathrm{B}$ to estimate emission heights as describe in the next chapter. As we see in Section 2.2.1, the pulse profile evolution is somewhat similar in both bright phases. Therefore, we use the above best-fit beam parameters from BP1 in our emission height estimates for both bright phases.

We reported that the radio emission of $\mathrm{B}$ disappeared in 2008 March (see Section 2.2.2), because the line-of-sight precessed away from the partially radio-filled elliptical beam. According to the same argument that the beam is not entirely radio loud, we can explain the radio emission disappearance and reappearance towards our line of sight with this 3D elliptical beam geometry. Accordingly, the 3D model describes the reappearance is predicted to occur in around 2024 with the same part of the beam. Therefore, Figure 2.15 changes with the partially-filled horse-shoe beam to non-detectable emission from 2008 to 2024. In contrast, the 2D model predicted the reappearance in around 2035 with the same part of the beam or in around 2014 if the beam has two symmetric radio-filled portions. If our new beam model is correct, then the beam should not have two symmetric radio-filled parts, because our lineof-sight crossed around the center of the beam when the disappearance occurred in 2008. Therefore, if there are two symmetric parts, then we would be able to detect radio emission at present day. The two different predictions for the reappearance from the two models mainly occur due to two different best-fit $T_{0}$ values. These two 
different $T_{0}$ values give two different solutions for the geometry of $\mathrm{B}$, however, the best-fit values for parameters $\alpha$ and $\theta$ are the same for the two models within the $2-\sigma$ uncertainty.

\subsection{Discussion}

In binary pulsars B1913+16 (Weisberg et al., 1989; Kramer, 1998; Weisberg \& Taylor, 2005), B1534+12 (Arzoumanian, 1996; Stairs et al., 2004), J1141-6545 (Hotan et al., 2005; Manchester et al., 2010), and J1906+0746 (Lorimer et al., 2006a), the long-term pulse profile evolution has been interpreted as the variation of the radio beam orientation with respect to our line-of-sight due to geodetic precession. With our observation of J0737-3039B, it is evident that the pulsar showed a dramatic evolution in its emission properties with respect to our line-of-sight. The observed bright single-peak pulse profile evolved to a double-peak pulse profile over time while reducing the single-to-noise significantly. During this evolution, the mean flux density decreased dramatically and reached almost zero around March 2008. We can explain this radio emission disappearance towards our line-of-sight with geodetic spin precession. This phenomena forces the spin axis of pulsar B to move around the orbital normal. Thus, with time, the spin axis of B has gradually precessed to a direction so that the spinning radio beam does not intersect our line-of-sight. However, in the future, the radio beam will gradually re-enter our line-of-sight due to spin precession.

We model the profile evolution of pulsar B with three different beam shape 
models based on geodetic spin precession: (a) circular beam model; (b) 2D elliptical beam model; (c) 3D elliptical beam model. Among these models, the geometry determined from elliptical beam models are consistent with each other, and also with the results of Breton et al. (2008) (see Table 2.1). We use the 3D elliptical beam model as our reference model to interpret pulsar B's geometry, because this configuration is more realistic and it provides the size of the beam.

The special choice of the beam construction with different intensity levels explains the single-peaked to double-peaked pulse profile evolution. On the first day, MJD 52997, the observed bright single-peaked pulse profile can be explained as our line-of-sight just grazing the outer edge of the maximum intensity level of the beam. When the line-of-sight gradually moves inward with time (i.e., towards the spin axis; see Figure 2.14(b)), the two-peaked pulse profile is formed since the maximum intensity level of the beam crosses our line-of-sight twice. However, the observed decreasing flux density over time cannot be explained by either model, since the intensity is constant along the cross-section of a hollow cone of the conical beam, in both the circular or elliptical cases. We believe the observed decrease in flux density must be due to a gradient in brightness across the beam or due to the changing influence of $\mathrm{A}$ on $\mathrm{B}$ during the span of these data. The distance between the two pulsars changes over time relative to our line-of-sight due to the relativistic advance of periastron of $17^{\circ} \mathrm{yr}^{-1}$ of the system. This results in a different amount of impact on B's emission by A with respect to Earth and leads to a change in the shape of the horse-shoe beam slightly, but this is likely a small effect, and not easy to model. Thus we ignored this effect. 
Despite the unusual modulation of B's emission by A, the 3-5\% duty cycle of B's pulse and the pulse profile shapes throughout the evolution are similar to those of pulsars with similar periods, with the dramatic geodetic precession of B offering us the chance to sample a much larger portion of the beam than typically possible. It is difficult to determine what is responsible for B's flux density variation. It may be that most pulsars have instrinisic intensity variations across their beams. Or, the decrease in the intensity of B's emission with time may be solely due to B's magnetosphere shape changing with the varying influence of A. Theoretical modeling of the influence of A's wind with time is necessary to address this. Our horse-shoe shaped beam fits the standard conal emission model (Rankin, 1983a), with the observed single to double peak evolution due to sampling different lines-of-sight across the conical beam, but this model does not explain the decreasing flux density and eventual disappearance of the pulsar. Pulse profile shapes have also been explained through the 'patchy' beam model (Lyne \& Manchester, 1988) in which intensities across the beam vary widely and often only a portion of the cone is visible. Observations of relativistic binary pulsar system J1141-6545 (Manchester et al., 2010) show that the emission across the beam is asymmetric with respect to the magnetic axis, consistent with a patchy beam. It is more difficult to interpret the evolution of B's profile with the patchy beam model, however, unless the patches are large enough to explain both components disappearing at the same time. Continued monitoring and sensitive searches for emission from B will provide invaluable information on the true beam shape of this pulsar. 


\section{Chapter 3}

\section{The emission altitudes of J0737-3039B}

This chapter presents the emission altitude estimates of J0737-3039B (pulsar B). We use a static dipole field line structure for pulsar B and determine its magnetosphere boundary by using a wind-magnetosphere interaction model (bow-shock model). Then we use this distorted magnetosphere structure to estimate the emission altitudes. We find that the emission altitudes vary across a single orbit due to the change in orientation of the bow-shock with respect to our line of sight. Further, we find the emission altitudes of pulsar B vary over time due to spin precession.

The work in this chapter was originally published as

Perera B. B. P., Lomiashvili D., Gourgouliatos K. N., McLaughlin M. A., Lyutikov M., 2012, ApJ, 750, 130; PSR J0737-3039B: A probe of radio pulsar emission heights

\subsection{Introduction}

As described in Section 1.3.1, the mechanism of pulsar radio emission and its origin within the pulsar magnetosphere are not well understood. In general, it is thought to be due to coherent radiation from relativistic plasma streaming along open magnetic field lines. Radio emission height estimates can constrain the 
emission mechanism to some extent (see Section 1.5). However, this is limited in isolated pulsars, because we observe only a small section of the magnetosphere due to an unchanging line-of-sight.

Pulsar B provides an excellent opportunity to study different emission regions due to its high relativistic spin precession, allowing us to observe different portions of the magnetosphere. We can also explore magnetospheric distortion, which affects the observed emission pattern.

By considering the relative transverse velocities of the two pulsars A and B, $660 \mathrm{~km} / \mathrm{s}$ (Lyne et al., 2004), along with the eclipse length of pulsar A, the estimated size of B's magnetosphere is about $10 \%$ of its $\mathrm{R}_{\mathrm{LC}}$ of $\sim 1.3 \times 10^{10} \mathrm{~cm}$. This implies that the wind of $\mathrm{A}$ compresses the magnetosphere of $\mathrm{B}$ and disturbs its polar cap (Lyutikov, 2004). This is due to the small separation of the pulsars $\left(\sim 9 \times 10^{10} \mathrm{~cm}\right.$ or 2.9 lt-s $)$ and the large spin-down luminosity of $\mathrm{A}\left(5.8 \times 10^{33} \mathrm{erg} \mathrm{s}^{-1}\right)$ compared to B $\left(1.6 \times 10^{30} \mathrm{erg} \mathrm{s}^{-1}\right)$. This is analogous to the distortion of the Earth's magnetosphere due to the Solar wind.

The wind interaction with the magnetosphere of $B$ produces a bow shock between A and B; this is likely the boundary of the magnetosphere of B. The shape of this boundary depends on the orientation of the magnetic axis of B. Lyutikov (2004) constrained the stand-off distance, or the distance from B to the vertex of the bow shock, to be $3.5 \times 10^{9} \mathrm{~cm}$ if the bow shock interface is a perfect resistor and $4 \times 10^{9} \mathrm{~cm}$ if it is partially resistive. These estimates inferred that the magnetosphere of $\mathrm{B}$ is located deep within its light cylinder and the open and closed field lines have a more complicated structure than that of an isolated pulsar. Since the wind- 
interaction boundary model is very important to study the emission geometry of B, we derive it again in this chapter with some improvements. This model describes the shape of the boundary for any orientation of the magnetic axis and allows us to model the open and closed field line structure more accurately.

This results in a method to use the derived field line structure to estimate the radio emission heights of $\mathrm{B}$. Since the bow shock boundary is located deep inside the light cylinder, the correction due to rotation on the static dipole field is small. Therefore we assume a non-rotating dipole field throughout the model. In our method, we assume that the emission comes from the direction tangential to the local field lines. We also assume that the emission comes from above the polar cap region, consistent with the narrow single- and double-peaked radio profiles.

\subsection{Boundary model}

- This bow-shock boundary model of pulsar B was developed with a contribution from Dr. K. N. Gourgouliatos (Purdue University)-

Due to the distortion of the magnetosphere, the properties of pulsar B are different from those of normal isolated pulsars. In isolated pulsars, we can determine the size of the magnetosphere by modeling the open and closed field lines, given the size of their light cylinder. However, as mentioned earlier, the magnetosphere of B is located deep inside the light cylinder and the structure of the open and closed field lines is more complicated due to the distortion from A's wind. 
In the first step, we approximate the structure of the magnetosphere as a rotating vacuum dipole. Then we apply a simple model for the wind-magnetosphere interaction, as in Lyutikov (2004). The wind of A creates a dynamic pressure on the magnetosphere of $\mathrm{B}$. The magnetosphere of $\mathrm{B}$ creates a magnetic pressure which opposes the wind pressure of A. At some point, these two pressures equal each other; this interface is likely the boundary of the magnetosphere of B. This boundary can be used to calculate the last open and closed field lines. We derive an expression for this boundary by equating the two pressures,

$$
\vec{B}^{2}\left(\overrightarrow{r_{\mathrm{B}}}\right) /(8 \pi)=L_{\mathrm{A}} \cos ^{2}(\gamma) / 4 \pi c{\overrightarrow{r_{\mathrm{A}}}}^{2}
$$

where $\overrightarrow{r_{B}}$ is the distance vector of the boundary with respect to pulsar $\mathrm{B}, \vec{B}\left(\overrightarrow{r_{B}}\right)$ is the magnetic field of pulsar $\mathrm{B}$ at $\vec{r}_{B}, L_{A}$ is the spin-down luminosity of A, $\overrightarrow{r_{A}}$ is the distance vector of the boundary with respect to $\mathrm{A}$, and $\gamma$ is the angle between the normal to the boundary and $\overrightarrow{r_{A}}$ (see Figure 3.1). The relative pressures lead to a boundary much closer to B. For that reason we simplify the problem by setting the distance of the boundary with respect to A equal to the distance between the two pulsars and $\gamma$ equal to the angle between the normal to the boundary and the line connecting two pulsars. By assuming a magnetic dipole at the center of the coordinate system, we can write the magnetic field strength of the NS as $\vec{B}\left(\overrightarrow{r_{B}}\right)=\left(3 \hat{r}_{B}\left(\vec{m} \cdot \hat{r}_{B}\right)-\vec{m}\right) / r_{B}{ }^{3}$, where $\vec{m}=m(\cos \delta \cos \Omega t, \cos \delta \sin \Omega t, \sin \delta)$ is the magnetic moment, $\Omega$ is the rotational angular frequency, and $\delta$ is the angle between the magnetic moment and the line connecting the two pulsars (see Figure 3.1). 


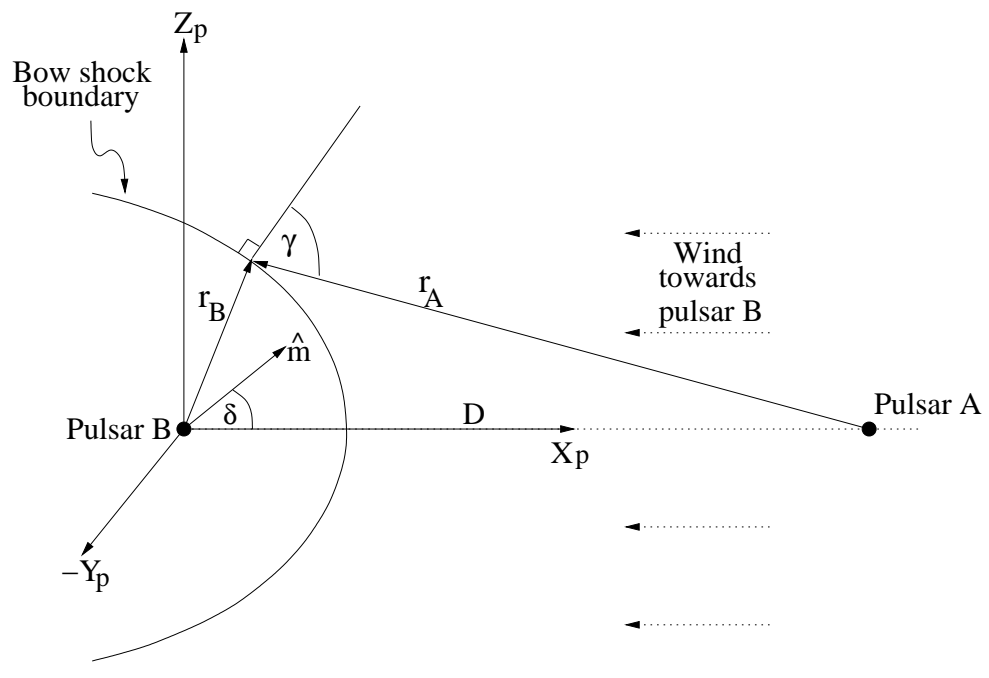

Figure 3.1: Geometry of the wind-magnetosphere interaction model. Pulsar A is located at a distance $\mathrm{D}\left(\sim 9 \times 10^{10} \mathrm{~cm}\right)$ away from $\mathrm{B}$ along the $x_{p}$-axis. The wind of A comes along the $-x_{p}$ direction and is shocked near $\mathrm{B}$. The physical interface has the shape of a bow shock and this is the boundary of the magnetosphere of B. $\overrightarrow{r_{B}}$ is the position vector at any point on the interface with respect to $\mathrm{B} . \overrightarrow{r_{A}}$ is the position vector of this point with respect to $\mathrm{A}$ and $\gamma$ is the angle between the normal to the boundary at this point and $\overrightarrow{r_{A}}$. $\delta$ is the angle between the magnetic moment axis $\hat{m}$ and the line connecting the two pulsars.

In isolated pulsars, we believe that the spin-down is caused by the power carried out along the magnetic field lines which are open with respect to the light cylinder (Contopoulos \& Spitkovsky, 2006). These open magnetic field lines start from the polar cap region of the NS surface and their shape changes with respect to the magnetic inclination. Spitkovsky (2006) proposed a realistic form of the spin-down luminosity of an isolated pulsar as a function of the magnetic inclination angle. However, the open field line structure of pulsar B is somewhat different than for an isolated pulsar and, therefore, we need to define the magnetic fields lines with respect to the bow shock boundary. Therefore, we modified the spin-down luminosity equation that is given in Spitkovsky (2006) by including the area of the polar cap region. This can be written as 


$$
L_{\mathrm{B}}=\Omega^{2} B_{0}^{2} S^{2}\left(1+\sin ^{2} \alpha\right) / 4 \pi^{2} c
$$

where $\Omega$ is the rotational angular frequency, $B_{0}$ is the polar magnetic field of pulsar $\mathrm{B}, S$ is the area of the polar cap region, and $\alpha$ is the magnetic inclination. As we mentioned earlier, with previous geometry models (Breton et al., 2008; Perera et al., 2010), $\alpha$ is taken to be $\sim 70^{\circ}$. Note that the original version of the equation (as in Spitkovsky, 2006) can be obtained by taking $S$ to be the area of the polar cap of a dipole as defined by the open magnetic field lines with respect to the light cylinder.

In order to determine the boundary, we solve equation (3.1) numerically and then use equation (3.2) to determine the value of the magnetic field. To do so, we simplified the problem to a $2 \mathrm{D}$ form in which the bow shock is represented by an equation involving $x_{p}$ and $z_{p}$ and lies on that plane. The equation of the bow shock then has the form $G\left(x_{p}, z_{p}\right)=f\left(z_{p}\right)-x_{p}$, which must be solved in order to determine the shape of the bow shock. Let the radial vector $\overrightarrow{r_{B}}=f\left(z_{p}\right) \hat{x}_{p}+z \hat{z}_{p}$ and the vector normal to the boundary $\vec{n}=\vec{\nabla} \cdot G\left(x_{p}, z_{p}\right)$. Then the dot product of these two gives the angle $\cos ^{2}(\gamma)=1 /\left(1+\left(d f / d z_{p}\right)^{2}\right)$ and this can be substituted in equation (3.1). According to our $2 \mathrm{D}$ form, we can write the magnetic moment $\vec{m}=m \cos \delta \hat{x_{p}}+m \sin \delta \hat{z_{p}}$ and then derive the magnetic field of $\mathrm{B}$ at distance $\overrightarrow{r_{B}}$, $\vec{B}\left(\overrightarrow{r_{B}}\right)$, as a function of $m, f\left(z_{p}\right), z$ and $\delta$. Then equation (3.1) reduces to a first order differential equation of $f\left(z_{p}\right)$ and the solution determines the shape of the bow shock. First we assume an initial value for the magnetic moment, $m$, of $\mathrm{B}$ with a possible magnetic orientation, $\delta$, and solve the problem to determine the shape 
of the bow shock. Then we find which are the last closed field lines defined with respect to the bow shock and finally the shape and the area of the polar cap.

We repeat the procedure for 16 values of the angle $\delta$ between the magnetic moment and the line connecting the two pulsars, evenly spaced between 0 and $\pi / 2$, and we find the area of the polar cap for each of those orientations. In the estimation of the average area of the polar cap, we have weighted appropriately the fact that some values of $\delta$ occur more frequently than others during an orbital period. Using the spin-down luminosity given in equation (3.2) with the timing-derived $L_{B}$, we find a new value for the magnetic field. We repeat this process with this new magnetic field until the value of the magnetic field converges. This happens after five to ten iterations for an initial guess of the magnetic field within a couple of orders of magnitude away from the convergence value. In order to represent the $3 \mathrm{D}$ version of the bow shock, we assume that it is axially symmetric around $x_{p}$.

According to the best solution, the magnetic field of $\mathrm{B}$ is constrained to be $B_{B}=6.4 \times 10^{11} \mathrm{G}$, which is about a factor of two lower than the timing-derived value $1.2 \times 10^{12} \mathrm{G}$ assuming a vacuum dipole with a magnetic inclination of $90^{\circ}$ (Lyne et al., 2004). This new estimate is more realistic as it accounts for the boundary of the magnetosphere as the bow shock and a realistic magnetic inclination. The stand-off distance is constrained to be either $3.8 \times 10^{9} \mathrm{~cm}$ or $4.5 \times 10^{9} \mathrm{~cm}$ for the cases when the magnetic axis is either normal or parallel to the line connecting the two pulsars, respectively. Thus, the size of the boundary depends on the orientation of the magnetic axis of pulsar B. Moreover, the shape of the bow shock depends on the orientation of the magnetic axis. The stand-off distance corresponds approximately 
to $1 / 3$ of the light cylinder, thus for these distances the relativistic modifications are minimal and do not change the value of the stand-off distance by more than a few percent. For that reason we have chosen to calculate it using a vacuum dipole model rather than a more complicated geometry that takes into account relativistic effects as in Deutsch (1955). For simplicity, we assume the boundary is axially symmetric around the vector connecting the two pulsars. A maximum deviation of roughly $20 \%$ of the actual shape from the symmetric case occurs when the angle $\delta$ is $90^{\circ}$. Therefore, the shape of the boundary is sensitive to an angle of $\delta$, having a range of $\left[0^{\circ}, 90^{\circ}\right]$. If the angle $\delta$ is greater than $90^{\circ}$, then the boundary considered the effective $\delta$ of $180^{\circ}-\delta$. For example, if $\delta$ is $100^{\circ}$, then the effective $\delta$ for the boundary shape is $80^{\circ}$. Therefore, with the assumption that the magnetic axis is nearly aligned with the line-of-sight at the radio emission detection, the effective angle $\delta$ is small $\left(\sim 5^{\circ}-35^{\circ}\right)$ in the orbital longitude region of BP2. Thus, the deviation of the boundary model from the actual geometry of the boundary is small and the assumption of a symmetric geometry is reasonable. However, the deviation in the orbital phase region of BP1 is significant due to large effective angles of $\delta$ $\left(\sim 35^{\circ}-85^{\circ}\right)$

To derive an expression for the physical shape of the boundary, we examine different shapes which fit our results. As a preliminary fit, a parabola is a good guess, but a fourth-order polynomial describes the boundary better, yielding the minimum chi-squared value when we fit to our results. The coefficients of this polynomial describe the variation and are functions of the angle $\delta$. The best-fit polynomial is 


$$
x_{\mathrm{p}}=a(\delta)+b(\delta)\left(y_{\mathrm{p}}^{2}+z_{\mathrm{p}}^{2}\right)+c(\delta)\left(y_{\mathrm{p}}^{2}+z_{\mathrm{p}}^{2}\right)^{2}
$$

with a B-centered coordinate system in which the $x_{p}$ axis is towards $\mathrm{A}$, the $z_{p}$ axis is normal to the orbital plane, and the $y_{p}$ axis completes the right-handed coordinate system (see Figure 3.1). The three axes have units in centimeters. The coefficients $a(\delta), b(\delta)$, and $c(\delta)$ are

$$
\begin{aligned}
& a(\delta)=\left(\frac{0.83-0.01 \delta-0.06 \delta^{2}-0.05 \delta^{3}+0.03 \delta^{4}}{1.83 \times 10^{-10}}\right) \\
& b(\delta)=\left(\frac{-0.46+0.04 \delta-1.43 \delta^{2}+1.96 \delta^{3}-0.64 \delta^{4}}{5.45 \times 10^{9}}\right) \\
& c(\delta)=\left(\frac{-0.48-0.03 \delta+2.15 \delta^{2}-2.47 \delta^{3}+0.74 \delta^{4}}{1.62 \times 10^{29}}\right)
\end{aligned}
$$

where the angle $\delta$ is in radians and has a range of $[0, \pi / 2]$. Since they are functions of $\delta$, the boundary changes slightly with spin and orbital motions, as well as over time due to precession.

This boundary model is valid only up to $5 \times 10^{9} \mathrm{~cm}$, or $\sim 40 \%$ of the $\mathrm{R}_{\mathrm{LC}}$, from B. Beyond this limit, the physical assumption of the dynamical pressure is incorrect because the wind pressure on the magnetic field should be zero when it is parallel to the boundary at large distances. Also we have assumed an undistorted magnetic field of B in the model and the distortions at large distances will be significant. 


\subsection{Tracing the dipole field lines}

Due to the wind interaction with the magnetosphere of $\mathrm{B}$, it is complicated to understand the structure of the open and closed field lines. To determine the polar cap region that is required for the emission height estimation, we calculate the last closed field lines by tracing them with respect to the derived boundary model.

As is standard, we treat the magnetosphere of $\mathrm{B}$ as a magnetic dipole. For an isolated pulsar, the last closed magnetic field lines are defined as those that just touch the light cylinder and the ones interior to the last closed field lines are considered open field lines. In our case the boundary is not the light cylinder but the bow shock, with the last closed field lines defined as those that just touch this bow shock. The polar cap region is defined by these particular field lines and the shape of it can be determined by the locations where these field lines cross the NS surface. Defining the polar cap is important since we think that the radio emission is produced above this region.

Unlike those of isolated pulsars, the magnetosphere of B is not symmetric around the magnetic axis due to the shape of the boundary. This can be clearly seen by tracing the last closed field lines. In order to trace the field lines, we use the dipole field line equation in polar coordinates

$$
\begin{aligned}
r & =r_{0} \sin ^{2}(\lambda) \\
\phi & =\phi_{0}
\end{aligned}
$$


where $r$ is the radial distance to a given point along the field line and $r_{0}$ is the fieldline constant, or equatorial distance of the field line from the magnetic axis. The angle $\lambda$ is the colatitude of a given point along the field line and $\phi_{0}$ is the azimuth angle, or the longitude of the given field line. Then the Cartesian components of a particular field line are written as

$$
\begin{aligned}
& x=r \sin (\lambda) \cos (\phi) \\
& y=r \sin (\lambda) \sin (\phi) \\
& z=r \cos (\lambda)
\end{aligned}
$$

where the $z$-axis of the coordinate system is aligned with the magnetic moment axis and the other two axes are co-rotating with the NS.

To include the misalignment of the magnetic axis and also account for the spin phase, we transform equation (3.6) to another frame where the $z$-axis is aligned with the spin axis. In this frame (see Figure 3.2(a)), the Cartesian components are

$$
\begin{aligned}
& x_{\mathrm{s}}=x \cos \alpha \cos \phi_{\mathrm{spin}}-y \sin \phi_{\mathrm{spin}}+z \sin \alpha \cos \phi_{\mathrm{spin}} \\
& y_{\mathrm{s}}=x \cos \alpha \sin \phi_{\mathrm{spin}}+y \cos \phi_{\mathrm{spin}}+z \sin \alpha \sin \phi_{\mathrm{spin}} \\
& z_{\mathrm{s}}=z \cos \alpha-x \sin \alpha
\end{aligned}
$$

where $\alpha$ is the angle between the magnetic axis and the spin axis and $\phi_{\text {spin }}$ is the 


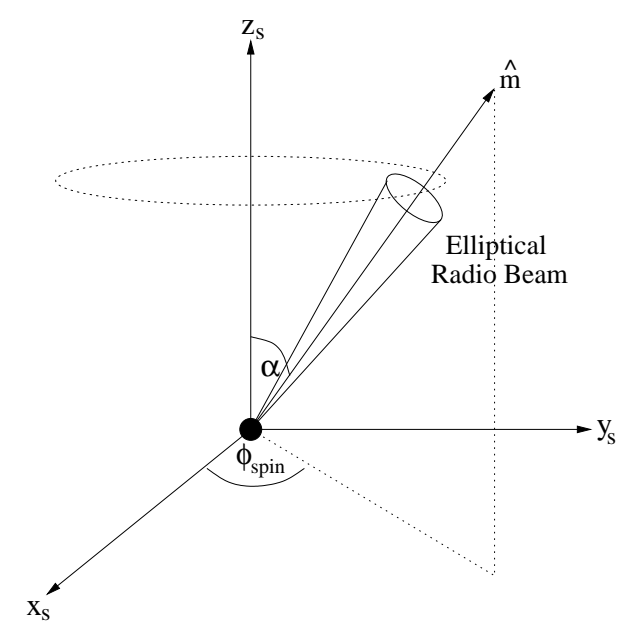

(a)

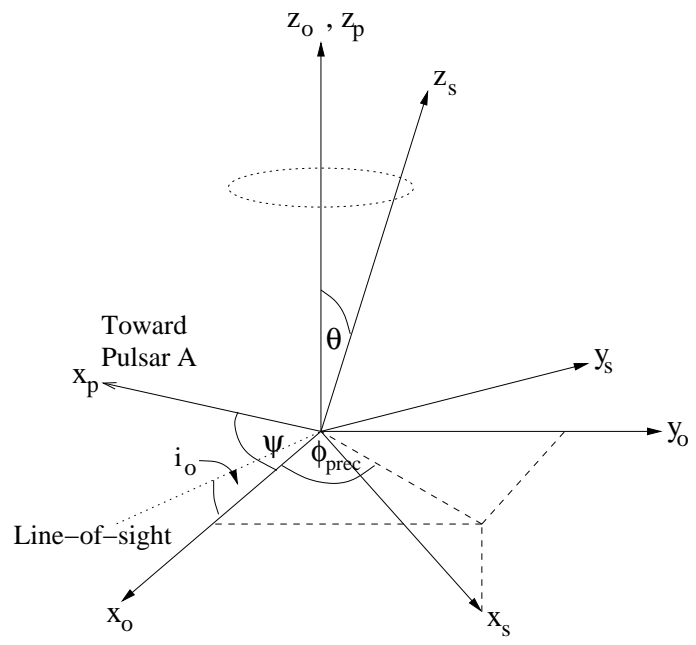

(b)

Figure 3.2: Cartesian coordinate systems that transform magnetic field lines from the co-rotating frame of the NS to the orbit-fixed frame. (a) The spin phase $\phi_{\text {spin }}$ and the misalignment of the magnetic axis $\alpha$ are defined in the frame $\left(x_{s}, y_{s}, z_{s}\right)$. The spin phase is measured from the $x_{s}$-axis. Therefore $\phi_{\text {spin }}=0$ is defined when the magnetic axis is in $x_{s}-z_{s}$ plane. (b) The colatitude of the spin $\theta$ and the precessional phase $\phi_{\text {prec }}$ is defined in the frame $\left(x_{o}, y_{o}, z_{o}\right)$. In this frame, the $x_{o}$-axis is in the plane of the $z_{o}$-axis and our line-of-sight (LOS). The spin precession $\phi_{\text {prec }}$ is measured from the $x_{o}$-axis. The frame of the bow shock boundary $\left(x_{p}, y_{p}, z_{p}\right)$ is then placed in this orbit-fixed frame with $z_{p} \| z_{o}$ and rotate with an angle of $\psi$ defined from the $x_{o}$-axis. Then the orbital phase is defined $\phi_{\text {orb }}=\psi+90^{\circ}$ as it measures from the ascending node. $i_{o}=90^{\circ}-i$, where $i$ is the orbital inclination.

spin phase. We measure the spin phase from the $x_{s}$ axis, which means that it is zero when the magnetic axis is in the $x_{s}-z_{s}$ plane.

The spin axis is also associated with the colatitude angle and the spin precessional phase. Geodetic spin precession changes the orientation of the spin axis with respect to our line-of-sight. In order to include the colatitude of the spin axis and effects of spin precession, we transform a particular field line to another frame which is fixed with our line-of-sight. We choose the frame with $z$-axis parallel to the orbital angular momentum axis and $x$-axis in the plane of the $z$-axis and the line-of-sight (see Figure 3.2(b)). The Cartesian components in this frame are 


$$
\begin{aligned}
& x_{\mathrm{o}}=x_{\mathrm{s}} \cos \theta \cos \phi_{\mathrm{prec}}(t)-y_{\mathrm{s}} \sin \phi_{\mathrm{prec}}(t)+z_{\mathrm{s}} \sin \theta \cos \phi_{\mathrm{prec}}(t) \\
& y_{\mathrm{o}}=x_{\mathrm{s}} \cos \theta \sin \phi_{\mathrm{prec}}(t)+y_{\mathrm{s}} \cos \phi_{\mathrm{prec}}(t)+z_{\mathrm{s}} \sin \theta \sin \phi_{\mathrm{prec}}(t) \\
& z_{\mathrm{o}}=z_{\mathrm{s}} \cos \theta-x_{\mathrm{s}} \sin \theta,
\end{aligned}
$$

where $\theta$ is the angle between the spin axis and the orbital angular momentum axis and $\phi_{\text {prec }}(t)$ is the spin precession phase measured from the $x$-axis (i.e., $\phi_{\text {prec }}(t)=0$ when the spin axis is in the plane of $\left.x_{o}-y_{o}\right)$. The spin precession phase is given by

$$
\phi_{\text {prec }}(t)=\Omega_{\text {prec }}\left(t-T_{0}\right)
$$

where $\Omega_{\text {prec }}$ is the spin precession rate of $\mathrm{B}$, which is $5.061(2)^{\circ} \mathrm{yr}^{-1}$, and $T_{0}$ is the time when the $\phi_{\text {prec }}(t)$ is zero, defined as the time when the spin axis is in the $x_{o}-y_{o}$ plane. By using the above set of equations, we can transform dipole field lines from the co-rotating frame of the NS to the orbit-fixed frame $\left(x_{o}, y_{o}, z_{o}\right)$ where the $x_{o}-y_{o}$ plane is in the orbital plane and $x_{o} \sin (i)$ is pointing towards the observer, where $i$ is the orbital inclination.

In order to place the polynomial boundary in the $\left(x_{o}, y_{o}, z_{o}\right)$ frame, we need to account for the orbital motion of B. Due to this motion, the orientation of the boundary changes with respect to our line of sight, because the location of A changes with respect to $\mathrm{B}$. This relative motion changes the shape of the magnetosphere of $\mathrm{B}$ with respect to the line-of-sight and then the shape of the polar cap region. This 
results a variation in the emission height estimate across the orbit (more details are given in section 3.4$)$. We place the boundary model $\left(x_{p}, y_{p}, z_{p}\right)$ in this orbit-fixed coordinate frame with $z_{o} \| z_{p}$ and then rotate it corresponding to the orbital phase $\phi_{\text {orb }}$ with $\left(x_{p} \cos \phi_{\text {orb }}-y_{p} \sin \phi_{\text {orb }}, x_{p} \sin \phi_{\text {orb }}+y_{p} \sin \phi_{\text {orb }}, z_{p}\right)$, where $\phi_{\text {orb }}=\psi+90^{\circ}$ (see Figure 3.2(b)). Here $\phi_{\text {orb }}$ is measured from the ascending node and $\psi$ is the rotation angle between $x_{o}$ and $x_{p}$ axes. Note that this $90^{\circ}$ angle is included to convert the rotation angle $\psi$ to orbital phase $\phi_{\text {orb }}$ as measured from the ascending node. Then we trace the last closed field lines according to the orientation of the boundary for the given orbital phase. For example, Figure 3.3 shows the confined magnetosphere in the boundary model with the last closed field lines on MJD 54050 (2006 November 11). Here, we use our best-fit geometry of the pulsar from the 3D elliptical beaming model that is described in section 2.3.3. At this particular epoch, the spin precession phase is $\phi_{\text {prec }}=46^{\circ}$ and we use the orbital phase $\phi_{\text {orb }}=223^{\circ}$ and the spin phase $\phi_{\text {spin }}=0$ in the figure. Note that, at this spin and the orbital phases, the spin axis of the pulsar is in the $x_{p}-z_{p}$ plane.

The spin of the magnetic axis also results in a change in the shape of the magnetosphere due to the misalignment of the magnetic moment. However, the most important orientation of the magnetic axis is when it reaches the closest approach to our line-of-sight (i.e. where we detect the emission). In order to measure the impact parameter $\beta(t)$, we use Equation 2.6. We calculate the spin phase which gives this particular closest approach, so that we can estimate the emission height only at this particular spin phase.

Now we can transform field lines from the co-rotating frame of the NS to the 

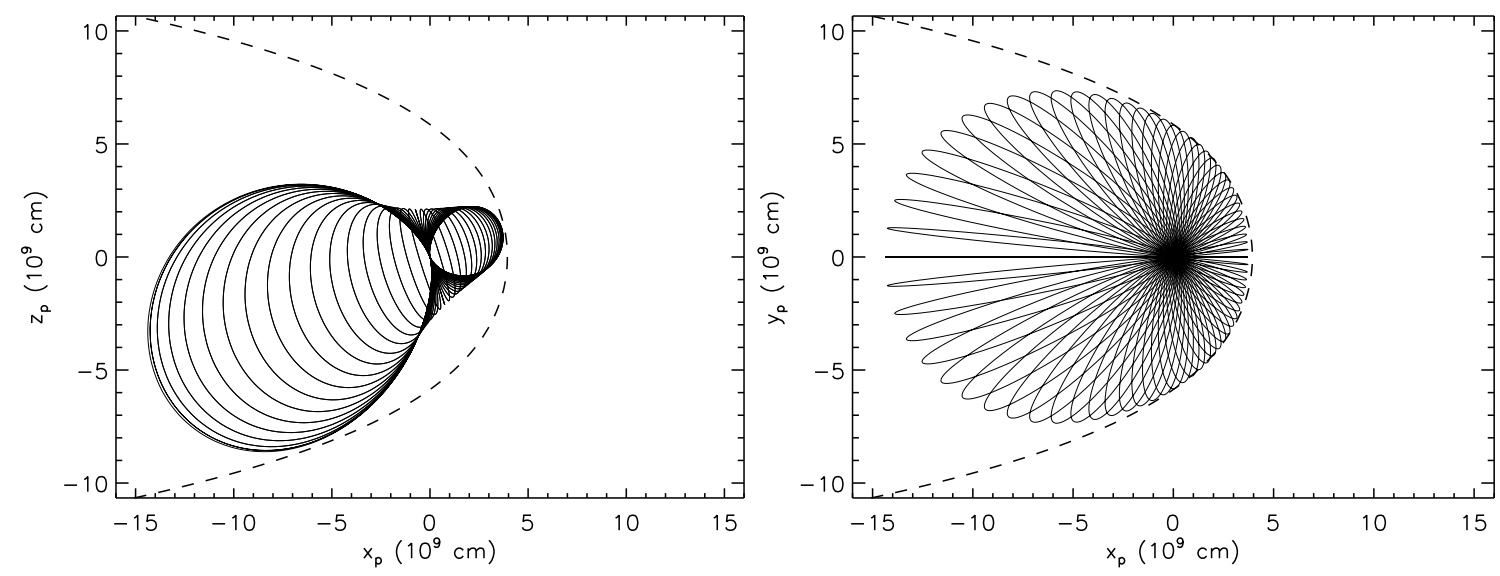

Figure 3.3: Confined rotating vacuum dipole in the boundary model on MJD 54050 (2006 November 11) - a view in the $x_{p}-z_{p}$ plane (left) and a view in the $x_{p}-y_{p}$ plane (right). Pulsar B is located at the center of the coordinate system and the wind of A comes towards the $-x_{p}$ direction. The dashed line shows the derived bow shock from the wind-magnetosphere interaction model and this models the open and closed field lines of the magnetosphere. The solid lines are the last closed field lines with respect to this boundary. The field lines that have higher latitude than these shown closed field lines are considered open field lines. The scale is in units of $10^{9} \mathrm{~cm}$. Here, $\alpha=61^{\circ}$ and $\theta=138.5^{\circ}$; these are our best-fit geometrical parameters from section 2.3.3. This view corresponds to the orbital phase of $223^{\circ}$. Note, for clarity of plots, we take the spin phase as zero, so that the north pole of the pulsar is pointing below the $z_{p}=0$ plane. However, the shape of the magnetosphere changes with the spin and orbital motion and over time due to precession. 
orbit-fixed frame. By using the boundary model, we can trace the last closed field lines, which determine the shape of the magnetosphere. Due to different orientations of the magnetic axis with spin, the shape of the magnetosphere with respect to our line-of-sight changes, but we are interested only in the spin phase which gives the closest approach to us. Nevertheless, orbital motion and spin precession change the shape and we need to account for these in emission height calculation.

\subsection{Emission height calculation}

In order to estimate the radio emission heights of pulsar $B$, we use the previously defined boundary model, the field line tracing technique, and the modeled geometry of the beam. As we mentioned in Section 1.3, the radio emission is assumed to be produced above the polar cap region and originates tangential to the local magnetic field lines.

First, we need to determine the boundary of the polar cap region, given by the last closed field lines. This can be done by tracing the field lines with the derived boundary model as described in Section 3.3. We assume that the radio emission comes from above the entire polar cap region, so that the outer edge of the pulse profile (i.e. $10 \%$ of the maximum intensity) corresponds to the region between the open and closed field lines, approximately equal to the last closed field line. Then we determine the emission height that originates from these last closed field lines.

In order to determine the tangent to a particular last closed field line at a given moment, we rewrite the coordinate transformations in section 3.3 in matrix form 
(see, e.g., Gangadhara, 2004), so that it is easy to evaluate the equations relevant for our calculation. First, we write the dipole field equation in the co-rotating frame of the NS as

$$
\vec{r}_{\text {cor }}=r_{0}\left(\sin ^{3} \lambda \cos \phi, \sin ^{3} \lambda \sin \phi, \sin ^{2} \lambda \cos \lambda\right)
$$

We then transform it to the orbit-fixed frame

$$
\vec{r}_{\text {orb }}=A \cdot B \cdot \vec{r}_{\text {cor }}
$$

where,

$$
A=\left(\begin{array}{ccc}
\cos \alpha \cos \phi_{\text {spin }} & -\sin \phi_{\text {spin }} & \sin \alpha \cos \phi_{\text {spin }} \\
\cos \alpha \sin \phi_{\text {spin }} & \cos \phi_{\text {spin }} & \sin \alpha \sin \phi_{\text {spin }} \\
-\sin \alpha & 0 & \cos \alpha
\end{array}\right)
$$

and

$$
B=\left(\begin{array}{ccc}
\cos \theta \cos \phi_{\text {prec }} & -\sin \phi_{\text {prec }} & \sin \theta \cos \phi_{\text {prec }} \\
\cos \theta \sin \phi_{\text {prec }} & \cos \phi_{\text {prec }} & \sin \theta \sin \phi_{\text {prec }} \\
-\sin \theta & 0 & \cos \theta
\end{array}\right)
$$

For the detection of radio emission, our line-of-sight must be parallel to the tangential vector of the given field line at a particular point. By locating this point on the field line, we can determine the height of the radio emission. To evaluate the tangent to the field line, we take $\vec{r}_{t}=\partial \vec{r}_{\text {orb }} / \partial \lambda$. Then the unit vector along the 
tangential direction $\left(\hat{r}_{t}=\vec{r}_{t} /\left|\vec{r}_{t}\right|\right)$ can be written as,

$$
\hat{r}_{\mathrm{t}}=A \cdot B \cdot \sqrt{\frac{2}{5+3 \cos (2 \lambda)}}\left(\begin{array}{c}
3 \cos \phi \sin \lambda \cos \lambda \\
3 \sin \phi \sin \lambda \cos \lambda \\
2 \cos ^{2} \lambda-\sin ^{2} \lambda
\end{array}\right) .
$$

The direction of the magnetic moment axis in the orbit-fixed frame can be written as

$$
\hat{m}=A \cdot B \cdot \hat{z}_{o}
$$

Then we evaluate the angle between the direction of the magnetic moment axis and the vector tangential to the field line $(\tau)$ at any time through the expression

$$
\cos (\tau)=\hat{r}_{\mathrm{t}} \cdot \hat{m}=\frac{1+3 \cos (2 \lambda)}{\sqrt{10+6 \cos (2 \lambda)}} .
$$

This is the same as equation (8) in Gangadhara (2004). At the point of detection of radio emission, we take the angle $\tau$ to be equal to the effective angular radius of the previously derived emission beam at the given time. According to our assumption that the outer edge of the pulse profile (10\% maximum) comes from the last closed field line, we take $\tau \approx \rho_{e, 10}(t)$, where $\rho_{e, 10}(t)$ is the effective angular radius of the beam (angle $A \hat{O} D$ of Figure 2.20 ) at the $10 \%$ of the maximum intensity level corresponding to a particular impact parameter $\beta(t)$. We can derive an equation for $\rho_{e, 10}(t)$ by using the spherical triangle $F A D$ of Figure 2.20 as 


$$
\cos \left(\rho_{e, 10}(t)\right)=\cos (\alpha) \cos (\alpha+\beta(t))+\sin (\alpha) \sin (\alpha+\beta(t)) \cos \left(w_{10}(t) / 2\right) .
$$

Here, $w_{10}(t)$, the model-estimated pulse width, can be found through equation (2.5) and (2.7) with the best-fit parameters $\alpha, \theta, \chi$, and $\rho_{a, 10}$ from the 3D elliptical beam model (see Section 2.3.3). The impact parameter $\beta(t)$ for the given time can be determined through equation (3.9) and (2.6) with the best-fit $T_{0}$. This $w_{10}(t)$ is simply the $10 \%$ pulse width of the 2D pulse profile given in Figure 2.15 at the given time. By simplifying equation (3.16), we find an expression for $\lambda$, which is the colatitude of the emission point. This expression can be written

$$
\cos (2 \lambda)=\frac{1}{3}\left[\cos \left(\rho_{e, 10}(t)\right) \sqrt{8+\cos ^{2}\left(\rho_{e, 10}(t)\right)}-\sin ^{2}\left(\rho_{e, 10}(t)\right)\right] .
$$

This is same as equation (9) in Gangadhara (2004), so that the colatitude angle of the emission point in our complicated geometry is simplified to that of an isolated pulsar. Then the emission height of this point can be calculated by using the first equation of (3.5). However, determining the field line constant, $r_{0}$, in this equation is difficult due to the bow shock boundary and its variation. Kijak \& Gil (1997) assumed that this $r_{0}$ is the $\mathrm{R}_{\mathrm{LC}}$ since the isolated pulsars that they have studied have low magnetic inclinations. To determine $r_{0}$ for our particular case, we trace the last closed field line which is tangent to our line-of-sight at the closest approach 
of the magnetic moment with respect to the bow shock boundary. Then we use the dipole field equation to estimate the emission height.

The orientation of the bow shock changes across the orbit with respect to our line-of-sight at the closest approach, so that the emission height changes with orbital longitude, because the last closed field lines are defined with respect to the bow shock. Moreover, when the central part of the radio beam crosses our lineof-sight, we will detect a double-peaked profile since the two edges, leading and trailing, of the beam cross our line-of-sight. Thus due to the different orientation of the magnetic moment axis at these two edges with respect to us, our line-of-sight is tangent to two different last closed field lines which have two different $r_{0}$ values. Thus the height of the emission produced by the leading and trailing edges of the beam are different. This is shown in Figure 3.4. For example, the emission heights produced by the leading components of the beam are constrained to be in a range of $[24 \pm 8,31 \pm 10]$ and $[20 \pm 6,21 \pm 7] \mathrm{R}_{\mathrm{NS}}$ on MJD 54050 (2006 November 11) for BP1 and BP2, respectively. The heights of the trailing edge of the beam in BP1 and BP2 are constrained to be in a range of $[15 \pm 5,19 \pm 6]$ and $[21 \pm 7,38 \pm 12] \mathrm{R}_{\mathrm{NS}}$, respectively. The errors of the height estimates are calculated from the 1- $\sigma$ uncertainties of the best-fit geometrical parameters from the beaming model. Thus, the uncertainty of the height estimate is in a range of $[6,10]$ and $[5,19] \mathrm{R}_{\mathrm{NS}}$ for the leading and the trailing edge of the beam, respectively, across the orbit on this particular day.

Also, due to precession of the spin axis, the emission height varies with time because the angle $\beta(t)$ varies with time. Again, there are two different heights for the leading and trailing edges of the beam. These are shown in Figure 3.5 and 


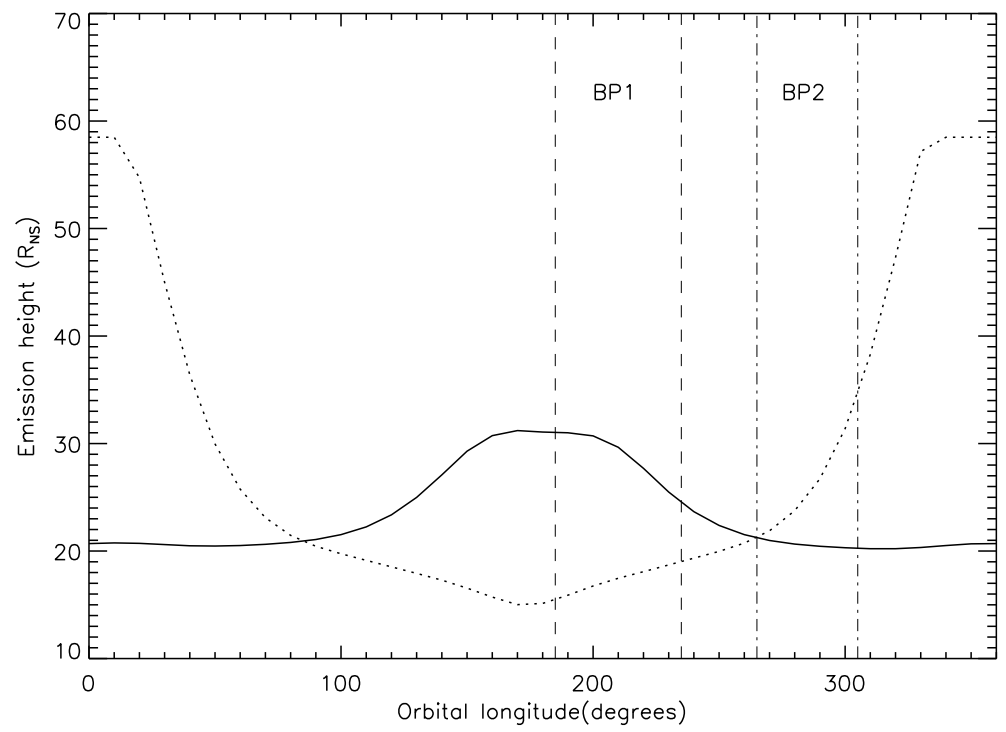

Figure 3.4: The model-estimated radio emission height across the orbit on MJD 54050 (2006 November 11). The solid line represents the height of the emission produced by the leading edge of the beam and the dotted line represents that for the trailing edge of the beam. In order to be consistent with observations, we used our best-fit geometrical parameters of the beaming model, $\alpha=61^{\circ}$ and $\theta=138.5^{\circ}$. At this epoch, $\phi_{\text {prec }}$ is $46^{\circ}$, the impact parameter $\beta$ is $3^{\circ}$ and the corresponding spin phase is $126^{\circ}$. Note that the difference between the corresponding heights for the leading and trailing components of the beam is more significant in some parts of the orbit. The orbital longitude regions for BP1 and BP2 are denoted with dashed and dot-dashed lines, respectively. 


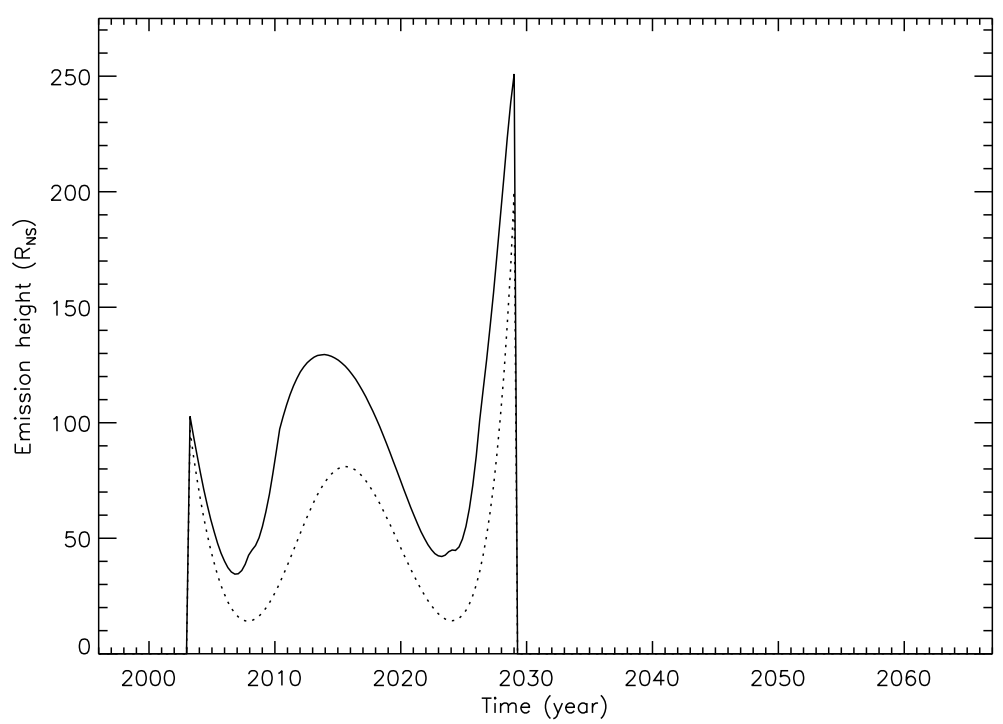

Figure 3.5: The model-estimated radio emission heights of the orbital longitude region of $\mathrm{BP} 1$ vs time across one precessional cycle. This predicted variation of emission height with time is due spin precession, making different line-of-sight cuts across the radio beam. The solid line represents the emission height from the leading edge of the beam and the dotted line represents that for the trailing edge. Here, the orbital phase is fixed at $200^{\circ}$ (BP1), but the spin phase changes with time corresponds to the $\beta$ value. The time axis represents a full precession cycle, $71 \mathrm{yr}$. Here, we have used the same geometrical parameters as in Figure 3.4 and assumed a full elliptical beam, not a partially filled horse-shoe beam. If the beam is partiallyfilled then no radio emission is expected from 2008 to 2024. This is why the model still predicts radio emission at present-day MJDs. The emission height is zero ( $2030-2067)$ when the line-of-sight is out of the radio beam.

3.6. In BP2, the difference between the two heights is not as significant as in BP1 because of the orbital position of $\mathrm{B}$ in $\mathrm{BP} 2$. In this region, pulsar $\mathrm{B}, \mathrm{A}$ and our line-of-sight are roughly aligned, resulting in less deviation in emission heights for the two edges of the beam.

If the emission is produced from the boundary between the open and closed field lines, we can consider these estimates to be the actual emission heights for B. If the emission is produced elsewhere within the open field line region, these are lower limits on emission heights. 


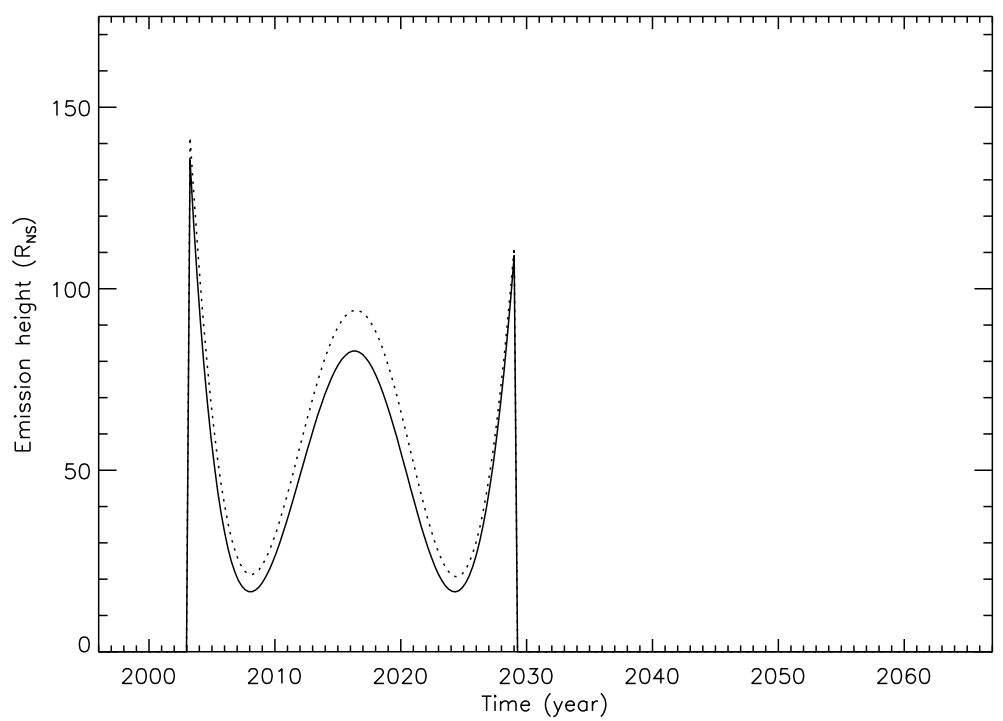

Figure 3.6: As described in Figure 3.5, but for BP2 (orbital longitude is $290^{\circ}$ ). Note that in this bright phase, the emission height difference between the leading and trailing components of the beam is not as significant as in BP1.

\subsection{Discussion}

The determination of radio pulsar emission heights is important for understanding their emission mechanisms. Pulsar B of the Double Pulsar provides a unique opportunity to study different emission regions of the magnetosphere due to precession. Also, the magnetosphere is distorted, exhibiting a complicated field line structure, due to the wind of A. These distortions depend on the orbital and rotational phases of $\mathrm{B}$. Observations of these distortions, not observed in isolated pulsars, via the orbital variations of the radio intensity of $\mathrm{B}$ allow us to pinpoint the location of radio emission.

We have applied a simple wind-magnetosphere interaction model to determine the boundary of the magnetosphere of B. The best solution describes the shape of the boundary as a polynomial, with coefficients dependent on the angle between the 
magnetic axis and the line connecting the two pulsars. Furthermore, the boundary was not axially symmetric, but for simplicity we modified it to be symmetric. The stand-off distance ranges from $3.8-4.5 \times 10^{9} \mathrm{~cm}$ according to the orientation of the magnetic axis with respect to the line connecting two pulsars, resulting in a size of the polynomial boundary of less than $30 \%$ of the $R_{\mathrm{LC}}$. However, this size is three times larger than the size inferred from eclipses of A. Thus, a possible explanation for this is that the particle density in the magnetosphere of B falls off significantly as a function of the radial distance from the center of the pulsar, so that the radiation of A penetrates the outer regions of B's magnetosphere.

Moreover, the variation of the boundary will change the shape of the open field line region. As a result, the spin-down luminosity of B can vary slightly due to the variation in the area of the polar cap. This causes a $1.5 \%$ periodic variation in the spin-down luminosity. It can also lead to a correction on the spin phase, but this is very small (Gourgouliatos et al., 2011). Thus we did not consider this effect in our model.

As we determined, the range of the allowed emission height depends on the orbital motion due to the relative orientation of the magnetic axis with respect to the boundary. Also, precession changes the location of the spin axis, so that the emission height changes with time. In both of these variations, we have been calculating the emission heights for both the leading and the trailing edges of the conal elliptical beam. For a CP with its light-cylinder boundary, these two edges give the same height due to cylindrical symmetry. When the impact parameter is equal to the angular radius of the beam across the semi-major axis, we would detect a single-peak 
profile, resulting in one emission height. Figure 3.4 shows that the relative heights of emission due to the two components of the beam switch in the two bright phase regions since the orientation of the boundary changes throughout the orbit. These estimated emission heights are about $1 \%$ of the $\mathrm{R}_{\mathrm{LC}}$ or $4 \%$ of the stand-off distance. Again, these should be considered lower limits if the emission does not originate on the last closed field line. Moreover, the analytical and numerical approaches to the upper limit estimate given in Perera et al. (2012) lead to the conclusion that pulsar B's radio emission is generated within $22 \%$ of the light cylinder.

In CPs, radio emission heights have been calculated by using their geometry and the pulse profile widths (Kijak \& Gil, 1997). The radio emission altitudes of CPs range from about 10 to $100 R_{N S}$, less than $10 \%$ of the $\mathrm{R}_{\mathrm{LC}}$ (see Section 1.5). Our emission height estimates are consistent with these results. Thus the radio emission produced by B likely has the same mechanism as for isolated pulsars, which is consistent with Lyutikov (2005).

The magnetospheres of pulsars can be distorted due to rotation as proposed in Dyks \& Harding (2004), resulting in a rotational sweepback of the magnetic field lines. They found that at low altitude the rotation deflects the local direction of the magnetic field line by at most an angle of the order of $\left(r / R_{L C}\right)^{2}$, where $r$ is the radial distance of the field line. We applied this rotational sweepback model to pulsar B along with our boundary model and found that the rotational sweepback is very small, because the deflection of the magnetic field line from its local direction is of order 0.1 radians. This is negligible compared to the distortions by the wind. However, this effect is significant when the radial distance of the field is close to the 
light cylinder, so that it is important in CPs.

We applied phase-shift method to the double-peaked pulse profiles of pulsar B to estimate the emission height (see Section 1.5). Because pulsar B has only a conal component, we assumed that pulse phase of zero was at the minimum between the two peaks. Then the phase-shift is measured from the two peaks, leading and trailing, with respect to this reference phase. The calculation shows that the phaseshift method does not work for pulsar B. For example, the emission height on MJDs 53860 and 53939 is 6 and $23 \mathrm{R}_{\mathrm{NS}}$, respectively. On MJD 54050 it is zero due to zero phase shift. Also on MJD 54400, the phase of trailing component is larger than the absolute phase of leading component, so that the emission height becomes negative. The reason for these calculated height fluctuations is that the pulse profile of $B$ is not stable and varies significantly. Therefore, it is difficult to measure the shift in pulse phase accurately. Also, as there are only two peaks in the pulse profile of $\mathrm{B}$, the determination of the pulse phase zero reference point is difficult. Therefore, the measured shifts and then the emission heights may not be correct, concluding that this method cannot be used to constrain emission heights of pulsar B. However, this is a useful method of estimating emission heights of CPs which have stable pulse profiles with both core and conal emission components (Gupta \& Gangadhara, 2003).

In summary, by using the method presented in this chapter, we can place limits on the radio emission height for any pulsar with well-determined emission geometry. The advantage of this method is that by estimating the field-line constant by tracing the magnetic field lines, we can constrain the emission heights of pulsars which have 
high magnetic inclinations that make them unsuitable for the other methods. Our radio emission height estimations for pulsar B will be useful for future studies and in particular can be used to constrain proposed geometrical models such as Lyutikov (2005) and Freire et al. (2009) in order to accurately explain the observations. 


\section{Chapter 4}

\section{The geometry of PSR J0737-3039A and its radio beam}

This chapter presents the geometry of pulsar A and its radio beam properties. Analyzing more than six years of data, we constrain the geometry of A from a simple double-pole circular beam model by fitting for the observed pulse profile widths over time, including the subtle changes at lower intensity levels. Then we develop a more advanced magnetosphere model based on a retarded vacuum dipole configuration and determine pulsar B radio beam geometry with a PC model, namely the beam size and the emission altitudes. Finally, we determine the full 3D geometry configuration of the Double Pulsar with the constrained pulsar A and B geometries.

The work in this chapter is in preparation to submit

Perera B. B. P., Kim C., McLaughlin M. A., Ferdman R. D., Kramer M., in preparation; Three-Dimensional Orbital Geometry of the Double Pulsar PSR J0737-3039

\subsection{Introduction}

As reported in Manchester et al. (2005) and Ferdman et al. (2013), pulsar A shows no significant variation in its pulse profiles over time due to its smaller spin misalignment angle (see Section 1.8 for a detailed discussion). Analyzing pulse widths at different intensity levels $(30 \%, 35 \%, 40 \%, 45 \%$, and $50 \%$ with respect to 
the peak), Ferdman et al. (2013) constrained the geometry of the pulsar. However, in order to include any subtle changes of the profile close to the baseline region, it is important to include more widths at lower intensity levels in the analysis. Therefore, in this study, we use $5 \%$ and $25 \%$ widths in addition to the other width levels that Ferdman et al. (2013) used and follow a similar method given in Section 2.3.3 to determine the geometry of pulsar A.

We constrain the pulsar and the radio beam geometries of A in two approaches. First, we use a simple double-pole circular beam model to explain the observed profile width and then constrain the pulsar geometry. Although it is useful to understand the pulsar beam geometry at some level, the simple double-pole circular beam model does not account for any magnetic field structures. Therefore, as a more realistic beam model, we use a PC beam model that can involve a retarded dipole magnetic field structure to fully describe the shape of the pulsar magnetosphere. As described in Section 1.3 in detail, pulsar magnetosphere models are mainly constructed at two limits: (a) a vacuum limit (Deutsch, 1955) and (b) a force-free limit with a plasma-filled magnetosphere (Spitkovsky, 2006). A true magnetosphere operates between these two limits (see Li et al., 2012; Kalapotharakos et al., 2012b). The force-free solutions are considered to be more realistic (Harding et al., 2008), but are computationally expensive to implement. Further, Harding et al. (2011) found the rotating dipole magnetosphere in vacuum provides better fits to observed highenergy pulse profiles compared to the results of force-free magnetosphere. Therefore, we incorporate a semi-analytic, widely used, vacuum retarded dipole magnetic field structure with a PC radio emission model to model the beam geometry of pulsar A. 


\subsection{Pulse Profiles of Pulsar A}

In this section, we describe our analysis of pulsar A's pulse profile in detail. We use the same data set that Ferdman et al. (2013) used in their analysis (from 2005 June (MJD 53524) to 2011 June (MJD 55721) at an observing frequency of $820 \mathrm{MHz}$ ). All pulse profiles are constructed with 2048 bins across the spin phase, resulting in a time resolution of $\sim 10 \mu \mathrm{s}$. As shown in Figure 1 of Ferdman et al. (2013), pulsar A's pulse profile has not significantly changed within that time span.

The brightest component (P1) is narrower than the secondary component (P2), see Figure 4.1. At each epoch, we calculate pulse widths of P1 and P2, separately, at different intensity levels $(5 \%, 25 \%, 45 \%$, and $65 \%)$ relative to each component's peak height. The uncertainties of these widths are calculated from the off-pulse rms. Note that we selected these particular intensity levels in order to reflect the width evolution in the analysis. For instance, we selected the $5 \%$ width to include the subtle changes of the profile appeared at lower intensity, so that we can model the boundary of the radio beam accurately. The other intensity levels were chosen selectively to avoid noise propertise such as the plateau region around $10 \%$ and the feature around $70 \%$ of the component P2, and similarly for P1 (see Figure 4.1). The profile becomes noisy at lower intensity, so that we selected $5 \%$ level as our lowest intensity to obtain the profile width. Figure 4.2 shows the pulse widths obtained from P1 (left panel) and P2 (middle panel) at 5\% intensity level. The measured widths vary only within $2^{\circ}$ for $\mathrm{P} 1$ and $0.5^{\circ}$ for $\mathrm{P} 2$ over time. Least-squared fits show that the $5 \%$ widths of $\mathrm{P} 1$ and $\mathrm{P} 2$ decrease in general with a rate of $0.1(7)^{\circ} \mathrm{yr}^{-1}$ and 


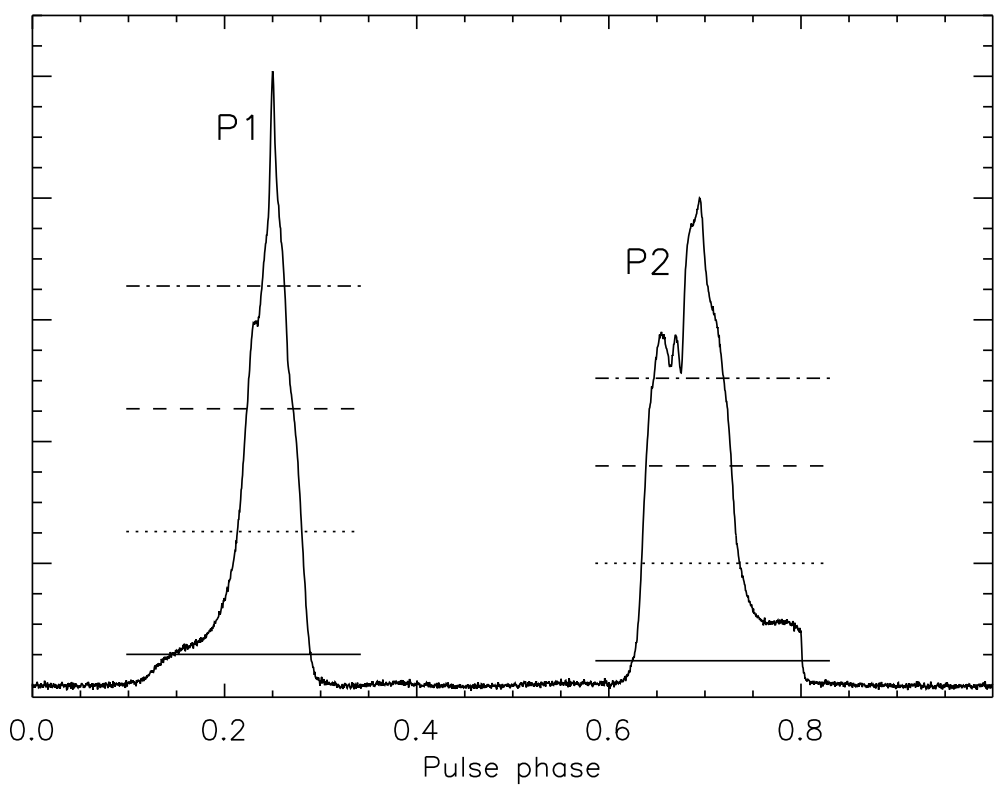

Figure 4.1: Integrated pulse profile of pulsar A on 6 May 2006 (MJD 53861) at $820 \mathrm{MHz}$ observing frequency. The primary narrower pulse component is denoted as P1 and the secondary broader component is denoted as P2. There are 2048 bins across the spin phase. Note that the fluctuations around pulse phase 0.4 and 0.6 are artifacts and not real. The different intensity levels with respect to each component's peak height are marked with horizontal lines, namely $5 \%$ (solid), $25 \%$ (dotted), $45 \%$ (dashed), and 65\% (dotted-dashed). The pulse widths are obtained with respect to these intensity levels.

$0.01(3)^{\circ} \mathrm{yr}^{-1}$, respectively. Therefore, within the errors, we find that no evidence for significant variation in pulse widths at $5 \%$ intensity over time, which is consistent with the results of other intensity levels in previous studies (Manchester et al., 2005; Ferdman et al., 2013).

In the right panel of Figure 4.2, we show the separation of the midpoints of $\mathrm{P} 1$ and $\mathrm{P} 2$ at 5\% intensity over time. Although the least-squared fit shows a decreasing trend with a rate of $0.2(2)^{\circ} \mathrm{yr}^{-1}$, the variation of the separation is consistent with zero. Therefore, P1 and P2 are separated by almost $180(4)^{\circ}$ at the $5 \%$ level, supporting the assumption that $\mathrm{A}$ is an orthogonal rotator and the two pulse components are due to seeing a radio beam from each pole of the NS. 

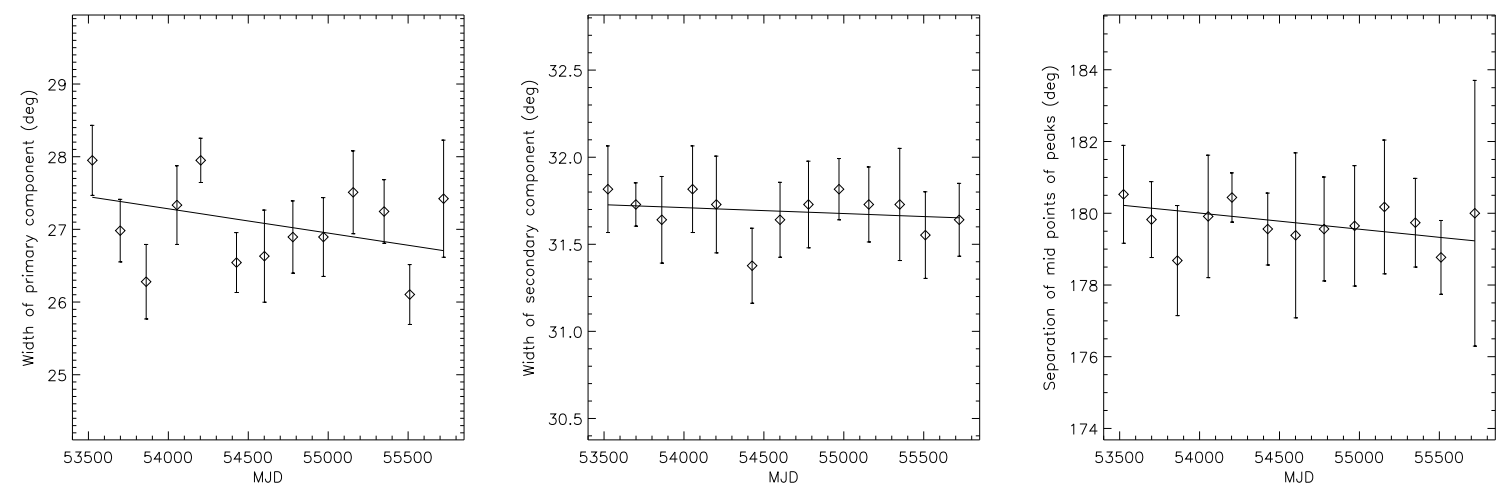

Figure 4.2: Pulse profile widths at $5 \%$ of their peak heights. The errors of these widths are computed from the off-pulse rms of pulse profiles. Left: Profile widths of the brightest component (P1) over time. Middle: Profile widths of the second brightest component (P2) over time. Right: The separation of the centers of the two components at their 5\% intensity level. The least-squared fitting to the data is shown as a solid line. We note that P1, P2, and the separation do not show a variation over time within their errors.

\subsection{Geometry of pulsar A with a simple circular beam}

Motivated by what we applied to pulsar B in Section 2.3.3, we fit a simple circular core radio beam model to the measured pulse widths from P1 and P2 at different intensity levels $\left(5 \%, 25 \%, 45 \%\right.$, and 65\%) and constrain $\alpha_{\mathrm{A}}$ and $\theta_{\mathrm{A}}$ independently of the line of sight.

Our method is similar to what was presented in Ferdman et al. (2013). The main difference between our work and theirs is that they calculated pairs of $\left(\alpha_{\mathrm{A}}, \theta_{\mathrm{A}}\right)$ for each intensity level. In contrast, we obtain a single pair of $\left(\alpha_{\mathrm{A}}, \theta_{\mathrm{A}}\right)$ considering all four intensity levels. Once we obtain $\alpha_{\mathrm{A}}$ and $\theta_{\mathrm{A}}$, we fix both these angles in order to estimate the half-opening angle of the beam $\rho_{\mathrm{A}}$ at the $5 \%$ intensity level. We assume the $5 \%$ intensity level is roughly the boundary of the beam, which reflects best the subtle change of the pulse profile widths.

Detecting a stable pulse profile over time implies that our line-of-sight always 
observes nearly the same cross section of pulsar A's radio beam. Therefore, as long as one is only concerned with global geometric angles such as $\alpha_{\mathrm{A}}$ and $\theta_{\mathrm{A}}$, the circular radio beam is a simple, yet valid, choice. Thus, we do not investigate other complex shapes such as an elliptical beam which was used for pulsar B where our line of sight cuts through significantly different parts of the beam in a surprisingly short-timescale of years (Section 2.3.3).

For a circular beam, as given in Gil et al. (1984), we can write the pulse width relation

$$
\cos \rho_{\mathrm{A}, \mathrm{j}}=\cos \alpha_{\mathrm{A}} \cos \left(\alpha_{\mathrm{A}}+\beta(t)\right)+\sin \alpha_{\mathrm{A}} \sin \left(\alpha_{\mathrm{A}}+\beta(t)\right) \cos \left(w_{\mathrm{j}}(t) / 2\right),
$$

where $j$ represents the different intensity levels, $\rho_{\mathrm{A}, \mathrm{j}}$ is the half opening angle of the beam at the $\mathrm{j}$-th intensity level, $\beta(t)$ is the impact angle, and $w_{\mathrm{j}}(t)$ is the pulse width measured at the given intensity level. Note that $\beta(t)$ and $w_{\mathrm{j}}(t)$ are functions of time. The impact angle is defined as $\beta(t)=\zeta_{\mathrm{E}}(t)-\alpha_{\mathrm{A}}$, where $\zeta_{\mathrm{E}}(t)$ is the angle between the spin axis and our line of sight at a given time (Equation 2.6). For general relativity, the spin precession rate $\Omega_{\text {prec }}$ is expected to be $4.8^{\circ} \mathrm{yr}^{-1}$ for pulsar $\mathrm{A}$ from Equation 1.26 (see Table 1.1).

Using these equations, we calculate the model-estimated pulse profile width $w_{\mathrm{j}}(t)$ at a given epoch $t$ for a given $\alpha_{\mathrm{A}}, \theta_{\mathrm{A}}, \rho_{\mathrm{A}, \mathrm{j}}$, and $T_{0}$. Then we fit the observed pulse width at the same epoch $t$ to the model-estimated width. We follow this method for measured pulse profiles at all epochs. By using a similar likelihood analysis as used in Section 2.3.3, we obtain $\alpha_{\mathrm{A}}, \theta_{\mathrm{A}}$, and $T_{0}$. During the fitting 
procedure, we use a single $\theta_{\mathrm{A}}$ and $T_{0}$ for both pulse components $\mathrm{P} 1$ and $\mathrm{P} 2$, assuming A is an orthogonal rotator. In other words, we assume P1 and P2 correspond to the north and south radio beams, respectively. Therefore, if the magnetic misalignment of the north beam which produces $\mathrm{P} 1$ is $\alpha_{\mathrm{A}}$, we can calculate the corresponding angle for $\mathrm{P} 2$ as $180^{\circ}-\alpha_{\mathrm{A}}$. Here, the half-opening angle $\rho_{\mathrm{A}}$ is a free parameter and we obtain it using two methods. As a first method (model M1), we assume that both beams have independent beam sizes, fitting the north $\left(\rho_{\mathrm{A}, \mathrm{N}}\right)$ and south $\left(\rho_{\mathrm{A}, \mathrm{S}}\right)$ beams for each parameter combination $\left(\alpha_{\mathrm{A}}, \theta_{\mathrm{A}}, T_{0}\right)$ until we get the maximum likelihood. After searching the entire parameter space, we obtain the best-fit values as follows: $\alpha_{\mathrm{A}}=88.1_{-0.6^{\circ}}^{\circ+3.0^{\circ}}, \theta_{\mathrm{A}}=0.9_{-0.9^{\circ}}^{\circ}$, and $T_{0}=$ MJD 61800 (see Table 4.3). The beam sizes $\rho_{\mathrm{A}, \mathrm{N}}$ and $\rho_{\mathrm{A}, \mathrm{S}}$ at the $5 \%$ intensity are $27.2(7)^{\circ}$ and $31.8(2)^{\circ}$ for $\mathrm{P} 1$ and $\mathrm{P} 2$, respectively.

For a static undistorted magnetosphere, both radio beams may have the same half-opening angles. Therefore, for the completeness of the analysis, we assume that both radio beams are similar in shape and size in the second method (model M2), i.e., we fit a single $\rho_{\mathrm{A}}$ for both $\mathrm{P} 1$ and $\mathrm{P} 2$. We then follow the same steps described in the previous paragraph. The best-fit parameters from M2 are $\alpha_{\mathrm{A}}=105.7_{-0.1^{\circ}}^{\circ}$, $\theta_{\mathrm{A}}=1.3_{-0.3^{\circ}}^{\circ}$, and $T_{0}=\operatorname{MJD} 54650$ (see Table 4.1). The best-fit $\rho_{\mathrm{A}}$ for M2 (at the $5 \%$ intensity) is $33.8(2)^{\circ}$. Note that our best-fit $\alpha_{\mathrm{A}}$ obtained from M2 is larger than that obtained from M1. This is due to the geometry of the pulsar with respect to our line of sight given that both of the radio beams are similar in size and the orbital inclination is less than $90^{\circ}$. Therefore, in order to generate the narrower pulse component $\mathrm{P} 1$, the radio beam should be located below the orbital plane (i.e., 
$\alpha_{\mathrm{A}}>90^{\circ}$ ), which is consistent with the best-fit $\alpha_{\mathrm{A}}$ from M2.

\subsection{Geometry of pulsar A with a retarded vacuum dipole PC model}

The circular radio beam model discussed in the previous section provides the information about the geometry $\left(\alpha_{\mathrm{A}}, \theta_{\mathrm{A}}\right)$ of the pulsar with beam size $\rho_{\mathrm{A}}$. In order to estimate the radio emission altitudes in detail, we need to account for the magnetic field line structure. In this section, we investigate the radio emission beam of A by applying a dipole magnetosphere configuration and assume that the $5 \%$ intensity levels of the profile, or wings, generates from the radio emission near the boundary of the last open and closed field lines

Following what was derived in Deutsch (1955) and used in Yadigaroglu (1997), Dyks \& Harding (2004) and Bai \& Spitkovsky (2010b), we model pulsar A's magnetosphere by a vacuum dipole field at retarded time $t_{\mathrm{r}}=t-r / c$. For a pulsar rotating around the z-axis with an angular velocity of $\Omega$ and magnetic inclination $\alpha_{\mathrm{A}}$, the time dependent magnetic moment is given as $\vec{\mu}(t)=\mu\left(\sin \alpha_{\mathrm{A}} \cos \Omega t \hat{x}+\right.$

$\left.\sin \alpha_{\mathrm{A}} \sin \Omega t \hat{y}+\cos \alpha_{\mathrm{A}} \hat{z}\right)$ in Cartesian coordinates. Then the magnetic field $\vec{B}$ of the retarded dipole is given as

$$
\vec{B}=-\left[\frac{\vec{\mu}(t)}{r^{3}}+\frac{\dot{\vec{\mu}}(t)}{c r^{2}}+\frac{\ddot{\vec{\mu}}(t)}{c^{2} r}\right]+\vec{r}^{2} \cdot\left[3 \frac{\vec{\mu}(t)}{r^{3}}+3 \frac{\dot{\vec{\mu}}(t)}{c r^{2}}+\frac{\ddot{\vec{\mu}}(t)}{c^{2} r}\right]
$$

where $r=|\vec{r}|$ is a radial distance to the emission point and $c$ is the speed of light (see Bai \& Spitkovsky, 2010b). As shown in Dyks \& Harding (2004), we can write the Cartesian components of $\vec{B}$ as follows 
Table 4.1: Geometric parameters of known pulsar binaries with geodetic precession. In this table: $\Omega_{\text {prec }}$ is the expected relativistic spin precession rate in general relativity, $\alpha$ is the magnetic misalignment angle, $\theta$ is the colatitude of the pulsar's spin axis, $\zeta_{\mathrm{E}}$ is the viewing angle, $\rho$ is the half-opening angle of the pulsar's radio beam, and $h$ is the radio emission altitude at the edge of the beam in $R_{\mathrm{NS}}$. Note that the first half of the table shows pulsar A's constrained geometric parameters based on several methods. The two values of $h$ correspond to those estimated for the beams from north and south magnetic poles.

\begin{tabular}{|c|c|c|c|c|c|c|c|}
\hline PSR name & $\begin{array}{c}\Omega_{\mathrm{prec}} \\
\left({ }^{\circ} \mathrm{yr}^{-1}\right)\end{array}$ & $\begin{array}{l}\alpha \\
\left({ }^{\circ}\right)\end{array}$ & $\begin{array}{c}\theta \\
\left({ }^{\circ}\right)\end{array}$ & $\begin{array}{l}\zeta_{\mathrm{E}} \\
\left({ }^{\circ}\right)\end{array}$ & $\begin{array}{c}\rho \\
\left({ }^{\circ}\right)\end{array}$ & $\begin{array}{c}h \\
\left(\mathrm{R}_{\mathrm{NS}}\right)\end{array}$ & Ref \\
\hline \multicolumn{8}{|l|}{ Pulsar A: from } \\
\hline M1 & 4.8 & $88(3)$ & $1(2)$ & {$[87.8,89.6]^{a}$} & 27,32 & 10,11 & $*$ \\
\hline M2 & - & $105(1)$ & $1.3(3)$ & {$[87.4,90.0]^{a}$} & 34 & 10,15 & $*$ \\
\hline $\mathrm{TPC}^{b}$ & - & $80(9)$ & $0^{c}$ & $86(14)$ & 31,32 & 10,11 & $*, 1$ \\
\hline $\mathrm{OG}^{b}$ & - & $88(17)$ & $0^{c}$ & $74(14)$ & 35,38 & 12,14 & $*, 1$ \\
\hline $\mathrm{RVM}^{b}$ & - & $99(8)$ & $0^{c}$ & $96(13)$ & 30,34 & 9,12 & $*, 1$ \\
\hline \multirow[t]{2}{*}{ Pulsar A } & - & $90(8)$ & $<6.1$ & - & $<90$ & - & 2 \\
\hline & - & $90(16)$ & $<2.3$ & - & $14,21^{d}$ & - & 3 \\
\hline Pulsar B & 5.1 & $61(8)$ & $138(5)$ & {$[49.8,132.8]$} & 14.3 & {$[15,38]$} & 4 \\
\hline B $1913+16$ & 1.21 & 156 & 21.1 & {$[111.7,153.9]$} & 12.4 & - & 5 \\
\hline B $1534+12$ & 0.51 & 102.8 & 25 & {$[52.2,102.2]$} & 4.87 & - & 6,7 \\
\hline J 1141-6545 & 1.36 & 160 & 93 & {$[20,166]$} & - & - & 8 \\
\hline J $1906+0746$ & 2.2 & $81(66)$ & $89(85)$ & - & - & - & 9 \\
\hline
\end{tabular}

${ }^{a}$ The angle $\zeta_{\mathrm{E}}$ is calculated from Eq. (2.6) with the best-fit values of other parameters.

${ }^{b}$ The pulsar geometric angles $\alpha, \theta$, and $\zeta_{\mathrm{E}}$ for TPC, OG, and RVM models are taken from Guillemot et al. (2013). The radio beam geometry $\rho$ and $h$ are estimated from our PC model as given in the text.

${ }^{c} \mathrm{TPC}, \mathrm{OG}$, and RVM results are based on $\theta_{A}=0^{\circ}$ as given in Guillemot et al. (2013).

${ }^{d}$ This $\rho$ is estimated for $25 \%$ intensity level.

Ref: (*) From this work; (1) Guillemot et al. (2013); (2) Ferdman et al. (2008); (3) Ferdman et al. (2013); (4) Perera et al. (2012); (5) Kramer (1998); (6) Stairs et al. (2004); (7) Thorsett et al. (2005); (8) Manchester et al. (2010); (9) Desvignes et al. $(2013)$ 


$$
\begin{array}{r}
B_{\text {ret, } \mathrm{x}}=\frac{\mu}{r^{5}}\left(3 x z \cos \alpha_{\mathrm{A}}+\sin \alpha_{\mathrm{A}}\left(\left[\left(3 x^{2}-r^{2}\right)+3 x y r_{\mathrm{n}}+\left(r^{2}-x^{2}\right) r_{\mathrm{n}}^{2}\right] \cos \left(\Omega t-r_{\mathrm{n}}\right)\right.\right. \\
\left.\left.+\left[3 x y-\left(3 x^{2}-r^{2}\right) r_{\mathrm{n}}-x y r_{\mathrm{n}}^{2}\right] \sin \left(\Omega t-r_{\mathrm{n}}\right)\right)\right) \\
B_{\text {ret,y }}=\frac{\mu}{r^{5}}\left(3 y z \cos \alpha_{\mathrm{A}}+\sin \alpha_{\mathrm{A}}\left(\left[3 x y+\left(3 y^{2}-r^{2}\right) r_{\mathrm{n}}-x y r_{\mathrm{n}}^{2}\right] \cos \left(\Omega t-r_{\mathrm{n}}\right)\right.\right. \\
\left.\left.+\left[\left(3 y^{2}-r^{2}\right)-3 x y r_{\mathrm{n}}+\left(r^{2}-y^{2}\right) r_{\mathrm{n}}^{2}\right] \sin \left(\Omega t-r_{\mathrm{n}}\right)\right)\right) \\
B_{\text {ret }, \mathrm{z}}=\frac{\mu}{r^{5}}\left(\left(3 z^{2}-r^{2}\right) \cos \alpha_{\mathrm{A}}+\sin \alpha_{\mathrm{A}}\left[\left(3 x z+3 y z r_{\mathrm{n}}-x z r_{\mathrm{n}}^{2}\right) \cos \left(\Omega t-r_{\mathrm{n}}\right)\right.\right. \\
\left.\left.+\left(3 y z-3 x z r_{\mathrm{n}}-y z r_{\mathrm{n}}^{2}\right) \sin \left(\Omega t-r_{\mathrm{n}}\right)\right]\right) .
\end{array}
$$

Here $r_{\mathrm{n}} \equiv r / R_{\mathrm{LC}}$, where $R_{\mathrm{LC}}$ is the light cylinder radius. Using pulsar A's spin period $\left(P_{\mathrm{s}}=23 \mathrm{~ms}\right)$, we fix pulsar A's light cylinder radius to be $R_{\mathrm{LC}}=c P_{\mathrm{s}} / 2 \pi=$ $1098 \mathrm{~km}$ in the calculation. Then the ratio $r_{\mathrm{n}}$ is small in the vicinity of the NS surface and the retarded field configuration is almost the same as the static field configuration in the 'near' zone (i.e., $r<<R_{\mathrm{LC}}$ ). As explained in Dyks \& Harding (2004), the location of any corotating point within the magnetosphere does not depend on time. In other words, the retarded magnetic dipole field configuration is fixed in space and time in corotating frame. Although, the field line structure rotates around the rotation axis as a whole with the pulsar spin.

As shown in Section 4.3 and also in Ferdman et al. (2013), pulsar A's spin colatitude $\theta_{\mathrm{A}}$ is almost zero. Thus, to simplify the model, we assume that pulsar A's spin axis is aligned with the orbital angular momentum $\left(\theta_{\mathrm{A}}=0^{\circ}\right)$. In order to determine the magnetic field lines, we use Eq. (4.3) with the fourth-order Runge-Kutta 
integration method. Two angles $\left(\theta_{\mathrm{m}}, \phi_{\mathrm{m}}\right)$ are used to define the footpoint of the magnetic field line on the NS surface, where we assume a NS radius of $R_{\mathrm{NS}}=10 \mathrm{~km}$. Here, $\theta_{\mathrm{m}}$ is the colatitude angle from the magnetic axis and $\phi_{\mathrm{m}}$ is the azimuth of the field line footpoint. Then we determine the field line which starts from this initial point. First, we determine the last closed field lines, by varying $\theta_{\mathrm{m}}$ for a given $\phi_{\mathrm{m}}$ until the field line becomes tangent to the light cylinder. We then define the PC region by calculating the footpoint of these last closed field lines on the NS surface. The shape of the PC region predicted by a retarded magnetic field is typically not symmetric around the magnetic axis and is dependent on $\alpha_{\mathrm{A}}$ (see Figure 2 in Dyks \& Harding, 2004). A field line with a smaller $\theta_{\mathrm{m}}$ is open with respect to the light cylinder and referred as an open field line. We then model these open field lines which are located within the $\mathrm{PC}$ region. In order to do that, we define a set of field line footpoint rings within the $\mathrm{PC}$ region with a fixed colatitude ratio of $\theta_{\mathrm{m}} / \theta_{\text {rim }}$, where $\theta_{\text {rim }}$ is the colatitude of the $\mathrm{PC}$ rim of a given $\phi_{\mathrm{m}}$. We calculate footpoints with a fixed $4^{\circ}$ increment in the azimuthal direction and obtain 90 field lines in each ring. Then, we define several sets of footpoint rings according to the colatitude ratio from 0.1 to 1 with an increment of 0.05 . We note that increasing the number of footpoints in a ring and the number of rings within the $\mathrm{PC}$ would smooth the modeled pulse profile and the shape variation becomes negligible. By testing several values, we found that the above given increments on these two parameters were sufficient for this analysis. Starting from these footpoints, we draw the open field lines using numerical integration (see Figure 4.3). Figure 4.3 shows the last closed field line structure of the vacuum retarded dipole magnetosphere. 

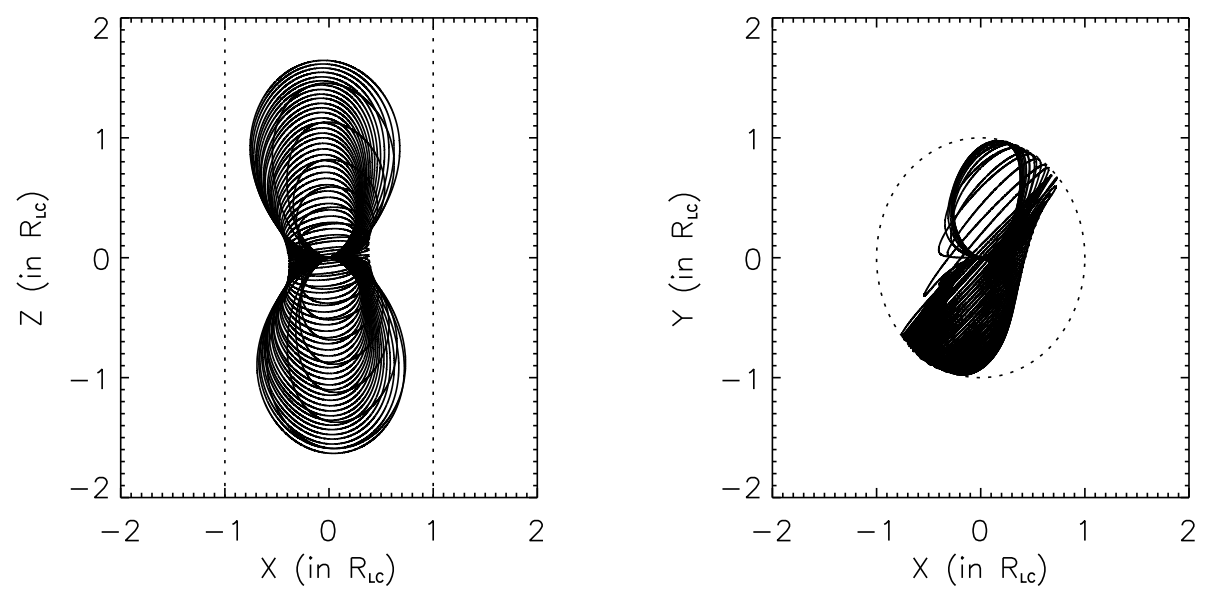

Figure 4.3: Retarded vacuum dipole field line magnetosphere for $\alpha=85^{\circ}$ in two different views. The last closed field lines are plotted in solid and the light cylinder represents in dotted line. All the axes are scaled in light cylinder radii $\mathrm{R}_{\mathrm{LC}}$.

The exact radio emission mechanism is not well understood, although we believe that the charged particles stream along magnetic field lines and emit radiation tangential to the local field line at the emitting point. Therefore, we first determine the photon emission direction at any given emission point on a field line in the PC region. In order to do that, we perform numerical integration with a fixed step size. By using a smaller step size, we can safely assume that the unit vector of the field line segment at a given point is indeed tangent to the field line. This guarantees that the unit vector of emitted photons $\left(\hat{\eta}^{\prime}\right)$ are also tangential to the field line at this point. The unit vector of photons are represented by two angles: the colatitude of the tangent from the rotation axis or the viewing angle $\zeta$ and the azimuth angle or the spin longitude $\phi$. Here, we consider the inertial observer frame, where the direction of the photon is not $\hat{\eta}^{\prime}$. In order to get the photon direction correctly in this frame $(\hat{\eta})$, we use the aberration formula (see Eq. (1) in Dyks \& Rudak, 2003) that accounts for the local corotational velocity with respect to the inertial observer 
frame as follows

$$
\hat{\eta}=\frac{\hat{\eta}^{\prime}+\left[\gamma+(\gamma-1)\left(\vec{\beta} \cdot \hat{\eta}^{\prime}\right) / \beta^{2}\right] \vec{\beta}}{\gamma\left(1+\vec{\beta} \cdot \hat{\eta}^{\prime}\right)}
$$

where $\gamma=\left(1-\beta^{2}\right)^{-1 / 2}$ is the Lorentz factor and $\vec{\beta}=(\vec{\Omega} \times \vec{r}) / c$ is the local corotation velocity in units of the speed of light at the emission point $\vec{r}$. Due to aberration, we observe emission slightly earlier in time, or in spin phase. The aberration is in particular important when the emission point $\vec{r}$ is large, i.e., the maximum aberration occurs close to the light cylinder radius with the maximum corotation velocity. Since pulsar A is nearly orthogonal as we found in Section 4.3, the corotation velocity of charged particles is important and hence, we include aberration in the model.

The next step is to include the photon propagation time delay between lowand high-altitude emission reaching the observer. This delay is given as $\vec{r} \cdot \hat{\eta} / R_{\mathrm{LC}}$ and is added to the aberration corrected azimuth of the emission point $\phi$ to get the correct phase of each photon (see Dyks \& Rudak, 2003). One of the important observable consequences of the delay is that trailing photons are piled up at a particular spin phase, giving rise to large number of photons ('caustic' regions) and producing emission peaks at the line-of-sight as the pulsar rotates. Both aberration and propagation time delay are most important in outer magnetospheric models that describe high-altitude emission, but they cannot be neglected at low altitudes.

We then map aberration and propagation delay corrected photons in a parameter space of $\zeta$ versus $\phi$, which is usually called a sky map. Figure 4.4 shows the photon map from the last closed field lines. We assume the coherent radio emission 


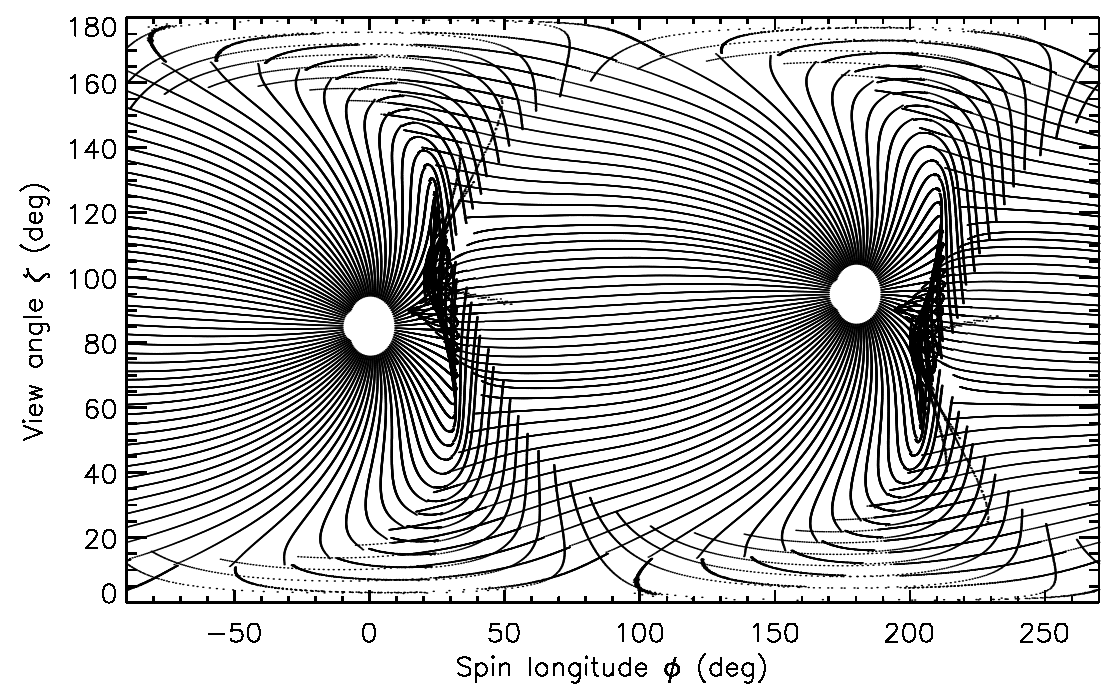

Figure 4.4: The projected photon map in the sky from the last closed field lines. The magnetic misalignment of the pulsar is $\alpha=85^{\circ}$. The photons are mapped starting from the NS surface up to a radial distance of one light cylinder radius. The two oval white regions are the north and the south PCs of the pulsar. Note that they are not completely circular. The two dark patches after the PCs are 'caustic' regions where the high-energy gamma-ray emission is enhanced. Note that the PC radio emission is produced only in a given region in this space.

is generated at a particular height above the PC region (see Figure 4.5). The modeled pulse profile is generated by limiting the photon emission to a particular region at this height above the PC (see Section 4.4.1 for more detail). Then, a horizontal cut of the sky map at a given viewing angle $\zeta$ returns the model pulse profile. By fitting the model pulse profile to pulsar A's observed profile, we can determine the radio emission altitude $h$ and the size of the radio beam $\rho_{A}$ based on the last closed field lines. This $\rho_{A}$ is an independent estimate for pulsar A's beam size from what we determined in Section 4.3 through a simple circular beam model. 


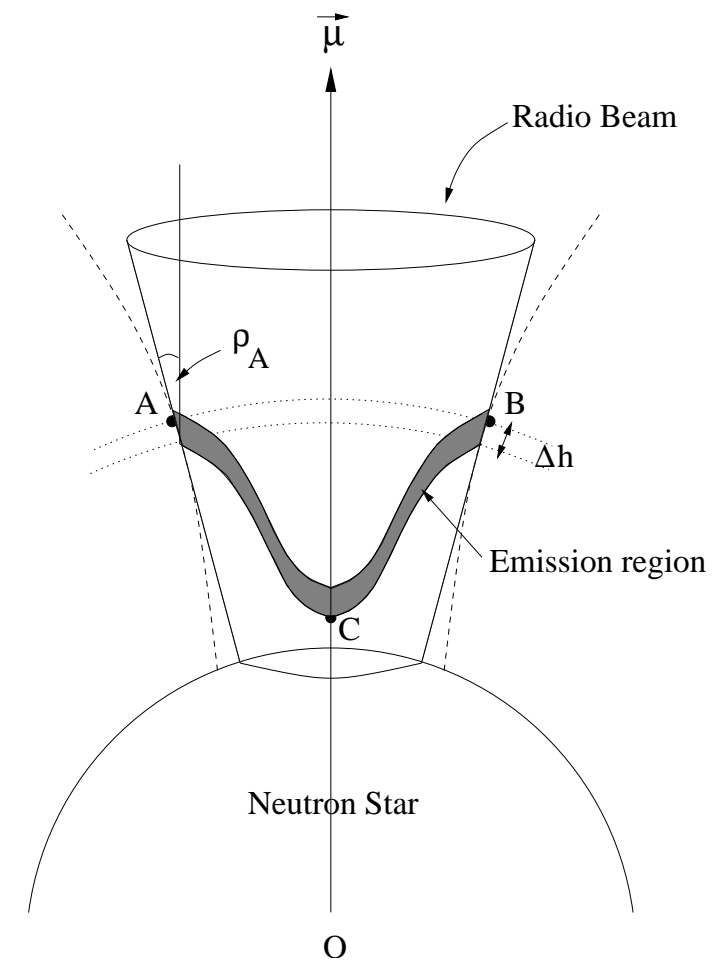

Figure 4.5: Schematic diagram of the radio emission beam (not in scale). Dashed lines are the two last closed magnetic field lines. The radio beam is tangent to these two field lines at point A and B. In other words, the radio beam is bounded by these two points. The coherent radio emission is generated within the filled area with thickness $\Delta h$. The colatitude of any given radio photon is less that the half-opening angle $\rho_{A}$ of the beam, i.e., all the photons are emitting within this boundary of the beam. The emission height at the edge of the beam $(h)$ represents from the length $O A(=O B)$. The emission height along the magnetic axis $\left(r_{0}\right)$ represents from the length $O C$. We assume the height of the emission region decreases exponentially from the beam edge towards the center. 


\subsubsection{Fitting Pulse Profiles of pulsar A}

As the PC region is bounded by the last closed field lines, we assume the outer edges (i.e., $5 \%$ intensity levels), or wings, of a pulse profile are generated from the emission within a thickness of $\Delta h$ along these last closed magnetic field lines at a emission altitude $h$ (see Figure 4.5). The inner part of the pulse component is assumed to be generated from the emission within the same thickness of $\Delta h$ along open field lines above the NS surface. If we fit the entire pulse profile including wings and the inner part of the pulse component at once, the higher intensity regions of the profile dominate the result, providing unrealistically large beam sizes and emission altitudes. Thus, we fit pulse profiles in two steps to avoid this issue.

The first step (Step One) is to estimate the emission altitude $h$ and the emission width $\Delta h$ which correspond to the profile wings. In order to do this, we map the photon emission from the last closed field lines by varying $h$ and $\Delta h$. Then we fit the modeled profile wings to the observed profile wings and obtain the best-fit $h$ and $\Delta h$ by minimizing $\chi^{2}$. We determine the half-opening angle or the beam size $\rho_{\mathrm{A}}$ of the radio beam from the direction of the photon emission at this best-fit $h$.

The second step (Step Two) is to model the entire region of the open magnetic field lines fixing the emission altitude to be the best-fit $h$ and $\Delta h$ obtained from Step One. We then compare the entire model pulse profile with the observed profile. However, using a single emission height from the edge of the beam is unrealistic as the emission is not necessarily generated at one particular altitude across the entire open field line region (Lyne \& Manchester, 1988). Therefore, in Step Two, 
we investigate different emission altitudes across the beam in addition to a constant emission altitude. In this model, we simply assume the radio emission altitude falls off exponentially with height towards the center of the beam from the edge (see Figure 4.5). Then we write an expression for the emission altitude across the beam as $r=h \exp \left(-\left(\rho_{\mathrm{r}}-\rho_{\mathrm{A}}\right)^{2} / 2 \sigma^{2}\right)$, where $r$ is the emission height at any point within the $\mathrm{PC}$ region. Again we assume that emission is generated within the thickness of $\Delta h$ at altitude $r$. We emphasize that $h$ is the emission height at the edge of the beam where the pulse profile wing is formed and is obtained from Step One. The parameter $\rho_{\mathrm{r}}$ is the colatitude of the photon at $r$ with respect to the pulsar's magnetic axis and can be obtained from the direction of the photon emission. Based on the definition, we have an inequality $\rho_{\mathrm{r}} \leq \rho_{\mathrm{A}}$. The parameter $\sigma$ determines the shape of the cross section of the beam and can be written as $\sigma=\rho_{\mathrm{A}} / \sqrt{-2 \ln \left(r_{0} / h\right)}$, assuming $\rho_{\mathrm{r}}=0^{\circ}$ along the magnetic axis at lower altitude. The height $r_{0}$ is the emission altitude at the magnetic axis. Once $h$ and $\rho_{\mathrm{A}}$ are obtained from Step One, we vary $r_{0}$ and fit the full model pulse profile to the observed profile as explained in Step Two and estimate the best-fit $r_{0}$. Instead of assuming two identical beams, we assume the emission altitudes at wings of north $\left(h_{\mathrm{N}}\right)$ and south $\left(h_{\mathrm{S}}\right)$ beams can be different and calculate each separately. Likewise, we define the emission altitude at the magnetic axis from the two beams as $r_{0, \mathrm{~N}}$ and $r_{0, \mathrm{~S}}$.

The required angle $\alpha_{\mathrm{A}}$ in the model is obtained from Section 4.3 and $\zeta_{\mathrm{E}}$ is calculated from Equation (2.6). Based on the geometries for models M1 and M2, we estimate two set of values for $h, \rho_{\mathrm{A}}$, and $r_{0}$. Finally, in addition to the beam geometry based on the simple circular beam given in Section 4.3, we also determine 
pulsar A's radio beam geometry based on the derived pulsar geometry from Fermi gamma-ray observations and radio polarization as given in Guillemot et al. (2013).

\subsubsection{Results}

The best-fit geometry of M1 yields that $\zeta_{\mathrm{E}}$ varies slightly in time due to spin precession with non-zero $\theta_{\mathrm{A}}$. However, the range of $\zeta_{\mathrm{E}}$ is very small, so that the choice of MJD does not affect the model pulse profile significantly. As we confirmed in Section 4.2, the pulse profiles of pulsar A do not show a significant time evolution. Therefore, we consider the observed pulse profile on MJD 53861 (Figure 4.1) as the time-independent observed pulse profile of $\mathrm{A}$ and obtained $h$ and $\Delta h$ by fitting the modeled pulse profile to this one. According to the geometry of M1, the best-fit radio beam parameters are estimated to be $h_{\mathrm{N}}=10 \mathrm{R}_{\mathrm{NS}}, h_{\mathrm{S}}=11 \mathrm{R}_{\mathrm{NS}}, \Delta h=1.5 \mathrm{R}_{\mathrm{NS}}$, and $r_{0, \mathrm{~N}}=r_{0, \mathrm{~S}}=1 \mathrm{R}_{\mathrm{NS}}$. The best-fit beam half-opening angles are $\rho_{\mathrm{N}}=31^{\circ}$ and $\rho_{\mathrm{S}}=33^{\circ}$. The best-fit pulse profile is shown in Figure 4.6. Note that the P1 component in modeled and observed profiles are consistent with each other due to its simple structure and the brightness. In general, this is a common feature in all our results explained below. Also, the aberration effect forms the leading step-like component of P1 easily and it is consistent with the observation. According to the best-fit solution of M2, the emission altitudes are estimated to be $h_{\mathrm{N}}=13 \mathrm{R}_{\mathrm{NS}}$ and $h_{\mathrm{S}}=15 \mathrm{R}_{\mathrm{NS}}$ with $\Delta h=1.25 \mathrm{R}_{\mathrm{NS}}$, and $r_{0, \mathrm{~N}}=r_{0, \mathrm{~S}}=1 \mathrm{R}_{\mathrm{NS}}$. Accordingly, the beam

sizes are estimated to be $\rho_{\mathrm{N}}=35^{\circ}$ and $\rho_{\mathrm{S}}=38^{\circ}$ (see Figure 4.6 for the best-fit pulse profile of M2). 


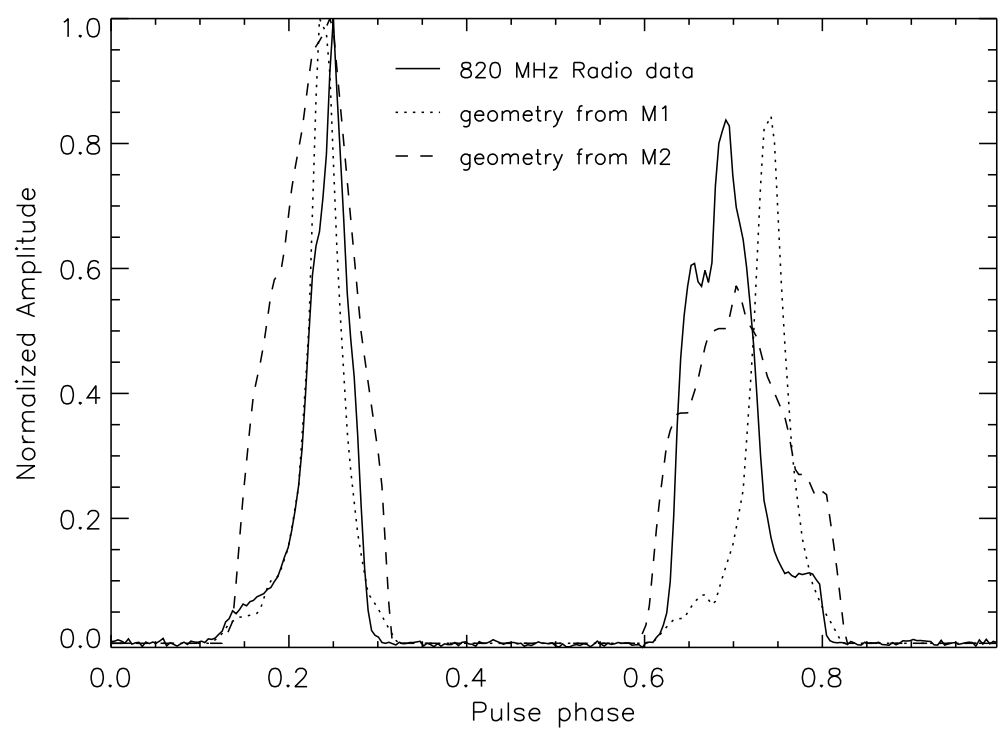

Figure 4.6: Modeled pulse profiles based on the circular beam model: M1 (dotted) and M2 (dashed). The observed integrated pulse profile at $820 \mathrm{MHz}$ is shown as a solid curve. Note that the observed profile has 256 bins across the spin phase. All profiles are normalized to the brightest peak obtained by each model. The emission altitudes for M1 and M2 are estimated to be between 10-15 $\mathrm{R}_{\mathrm{NS}}$.

Given that the pulse profiles of A show no evolution since its discovery, Guillemot et al. (2013) set $\theta_{\mathrm{A}}=0^{\circ}$ and fit TPC and OG emission models separately to gamma-ray light curves and derived the geometry of the pulsar (see Table 4.1 for the best results). The TPC model gives the best-fit model gamma-ray light curve at $\alpha_{\mathrm{A}}=80(9)^{\circ}$ and $\zeta_{\mathrm{E}}=86(14)^{\circ}$. With these parameters, we apply our PC beam model to search for the emission altitude and the beam size. The emission heights are estimated to be $h_{\mathrm{N}}=10 \mathrm{R}_{\mathrm{NS}}$ and $h_{\mathrm{S}}=11 \mathrm{R}_{\mathrm{NS}}$ with $\Delta h=0.75 \mathrm{R}_{\mathrm{NS}}$, $r_{0, \mathrm{~N}}=r_{0, \mathrm{~S}}=1 \mathrm{R}_{\mathrm{NS}}$, and the half-opening angles are $\rho_{\mathrm{N}}=31^{\circ}$ and $\rho_{\mathrm{S}}=32^{\circ}$. The OG model gives the best-fit parameters are $\alpha_{\mathrm{A}}=88(17)^{\circ}$ and $\zeta_{\mathrm{E}}=74(14)^{\circ}$. According to this geometry, our PC model estimated the emission heights to be $h_{\mathrm{N}}=12 \mathrm{R}_{\mathrm{NS}}$ and $h_{\mathrm{S}}=14 \mathrm{R}_{\mathrm{NS}}$ with $\Delta h=2 \mathrm{R}_{\mathrm{NS}}, r_{0, \mathrm{~N}}=r_{0, \mathrm{~S}}=1 \mathrm{R}_{\mathrm{NS}}$, and the half-opening angles to be $\rho_{\mathrm{N}}=35^{\circ}$ and $\rho_{\mathrm{S}}=38^{\circ}$. With these parameters, Figure 4.7 shows the 


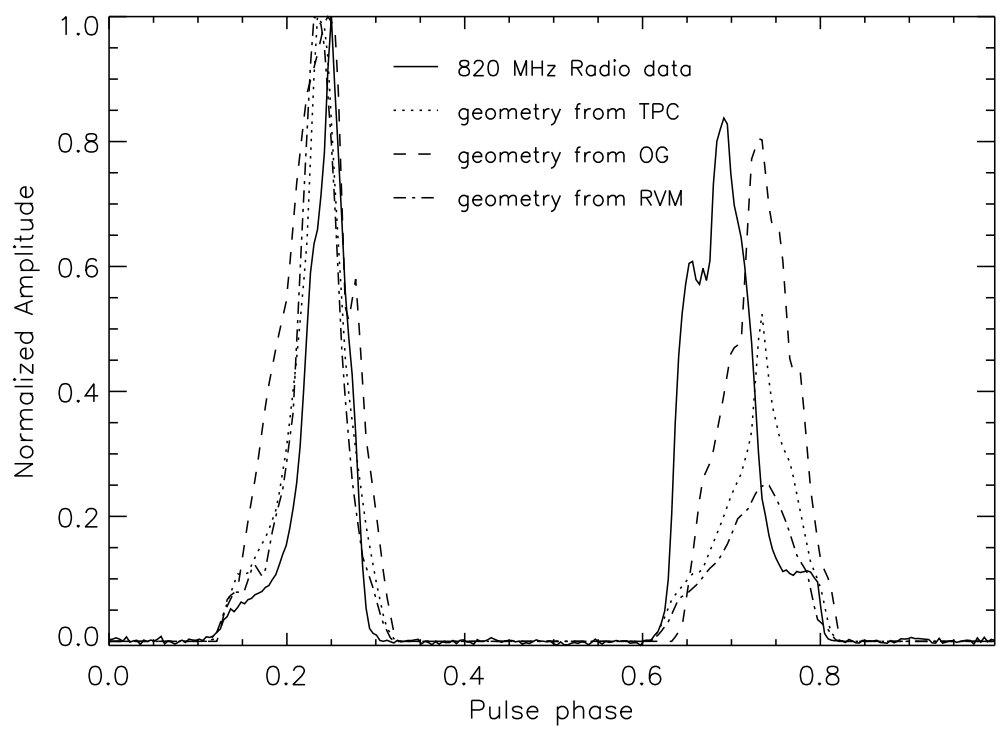

Figure 4.7: As described in Figure 4.6, but for the geometry derived from TPC (dotted), OG (dashed), and radio polarization along with RVM (dotted dashed). The estimated emission altitudes from these three models range between $9-14 R_{N S}$.

best-fit pulse profiles obtained from our PC model based on the pulsar geometries with two magnetosphere models (TPC and OG) we consider, in addition to M1 and M2, compared with the observed pulse profile of A.

Lastly, we include the radio polarization constrained angles of $\alpha_{\mathrm{A}}=99(8)^{\circ}$ and $\zeta_{\mathrm{E}}=96(13)^{\circ}($ Guillemot et al., 2013) in our PC model and estimate the radio beam properties. Based on this pulsar geometry, Figure 4.7 shows our best-fit PC model synthesized pulse profile with the emission heights of $h_{\mathrm{N}}=9 \mathrm{R}_{\mathrm{NS}}$ and $h_{\mathrm{S}}=12 \mathrm{R}_{\mathrm{NS}}$ with $\Delta h=1.25 \mathrm{R}_{\mathrm{NS}}, r_{0, \mathrm{~N}}=r_{0, \mathrm{~S}}=1 \mathrm{R}_{\mathrm{NS}}$, and the half-opening angles of the two beams are $\rho_{\mathrm{N}}=30^{\circ}$ and $\rho_{\mathrm{S}}=34^{\circ}$.

\subsection{The 3-D Orbital Geometry of the Double Pulsar}

In order to fully configure the orbital geometry of the Double Pulsar, we need the relative angle between the spin axes of the two NSs $\left(\Delta_{\mathrm{S}}\right)$. All other angles 
for $\mathrm{A}$ are obtained in the previous section and the main results are summarized in Table 4.3. Section 2.3.3 presented the geometry of pulsar B and Kim et al. (2013, submitted) presented its radio beam in detail.

Due to geodetic precession of the two pulsars (especially B), $\Delta_{\mathrm{S}}$ changes over time. Based on the results described in earlier sections, we are able to describes $\Delta_{\mathrm{S}}(t)$ by a simple equation as follows

$$
\cos \left(\Delta_{\mathrm{S}}(t)\right)=\cos \theta_{\mathrm{A}} \cos \theta_{\mathrm{B}}+\sin \theta_{\mathrm{A}} \sin \theta_{\mathrm{B}} \cos \left(\Delta \phi_{\text {prec }}(t)\right)
$$

where $\Delta_{\mathrm{S}}(t)$ is the relative angle between the spin axes of $\mathrm{A}$ and $\mathrm{B}$ at time $t$. The angles $\theta_{\mathrm{A}}$ and $\theta_{\mathrm{B}}$ are spin misalignment angles of $\mathrm{A}$ and $\mathrm{B}$ with respect to the orbital angular momentum. The angle $\Delta \phi_{\text {prec }}(t)$ is the relative spin precession angle and is defined by $\Delta \phi_{\text {prec }}(t)=\phi_{\text {prec }, \mathrm{A}}(t)-\phi_{\text {prec }, \mathrm{B}}(t)$, where $\phi_{\text {prec,A }}(t)$ and $\phi_{\text {prec }, \mathrm{B}}(t)$ are the spin precession phases of $\mathrm{A}$ and $\mathrm{B}$, respectively. Note that the angle $\Delta_{\mathrm{S}}(t)$ is not affected by the details of our assumptions on the pulsar radio beams or magnetic misalignment.

Figure 4.8 depicts the evolution of $\Delta_{\mathrm{S}}(t)$ over time for the two models M1 and M2. With this particular geometric framework, the minimum and maximum $\Delta_{\mathrm{S}}(t)$ are given by $\theta_{\mathrm{B}}-\theta_{\mathrm{A}}$ and $\theta_{\mathrm{B}}+\theta_{\mathrm{A}}$, respectively. Based on our results and the results in Section 2.3.3, $\theta_{\mathrm{B}}>\theta_{\mathrm{A}}$ (Table 4.1). We find that $\Delta_{\mathrm{S}}(t)$ changes periodically between $\left[137.6^{\circ}, 139.4^{\circ}\right]$ and $\left[137.2^{\circ}, 139.8^{\circ}\right]$ for M1 and M2 over time, respectively. This can be interpreted as the angle $\Delta_{\mathrm{S}}(t)$ varies by $1.8^{\circ}$ for $\mathrm{M} 1$ and $2.6^{\circ}$ for $\mathrm{M} 2$. At the current epoch, $\Delta_{\mathrm{S}}(t)$ for M1 and M2 are $138(5)^{\circ}$ and $137(6)^{\circ}$, respectively. As 


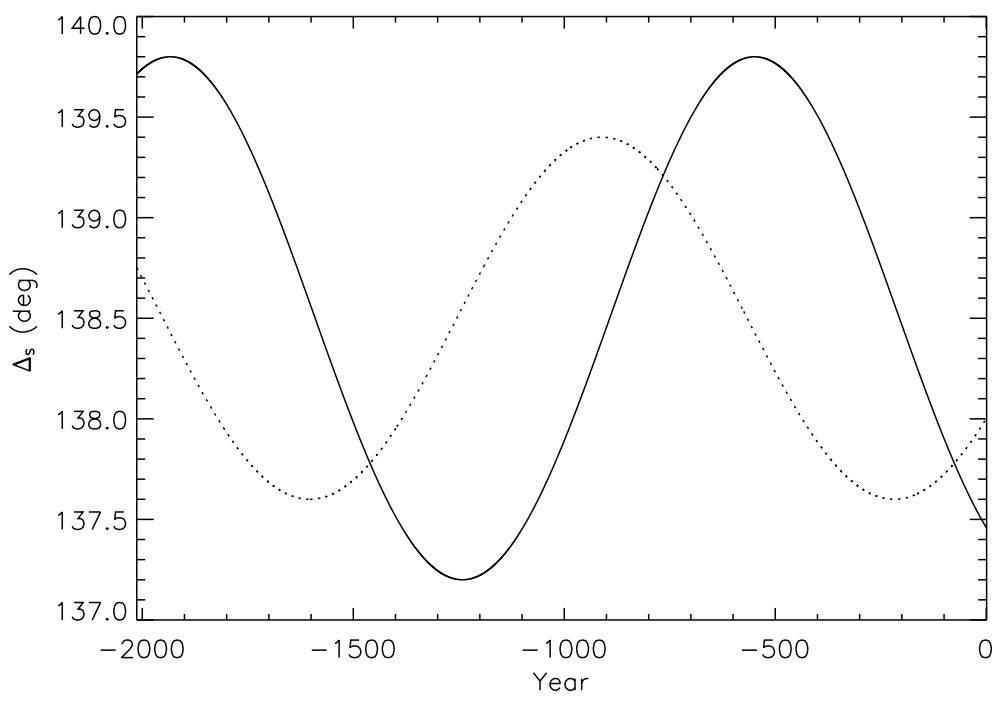

Figure 4.8: The variation of the relative angle between the spin axes of $\mathrm{A}$ and $\mathrm{B}$ pulsars over time for M1 (dotted) and M2 (solid). We compute $\Delta_{\mathrm{S}}(t)$ from the current epoch (year 0 in the x-axis) backwards in time. The period of the variation is about $1385 \mathrm{yrs}$, which is the beat period between the geodetic precession periods of 75 and 71 years for A and B pulsars.

expected from Equation $(4.5), \Delta_{\mathrm{S}}(t)$ is insensitive to different assumptions made in M1 and M2 and the results are different by only a couple of degrees in magnitude and by $\sim 400$ yrs in phase. The period of $\Delta_{\mathrm{S}}(t)$ is $1385 \mathrm{yrs}$. It corresponds to the beat frequency of $2.38 \times 10^{-11} \mathrm{~Hz}$, based on the spin precession periods of pulsar A (75 yrs) and pulsar B (71 yrs). Considering there is no mechanism to transfer angular momentum between the two NSs after the second supernova explosion and assuming the effects of magnetospheric interaction are negligible, we expect the evolution of $\Delta_{\mathrm{S}}$ of the Double Pulsar to follow Equation (4.5) until the two NSs merge.

Considering the $2 \sigma$ uncertainties of $\theta_{\mathrm{A}}$ and $\theta_{\mathrm{B}}$ given in Table 4.1 , we estimate the uncertainty of $\Delta_{\mathrm{S}}(t)$ to be $\pm 6^{\circ}$ for the two models. If $\theta_{\mathrm{A}}=0^{\circ}$ as suggested by Guillemot et al. (2013), $\Delta_{\mathrm{S}}(t)=\theta_{\mathrm{B}}=138.5^{\circ}$, which is a constant in time and does not show a periodic variation. We also note that, the estimated uncertainty 
of $\Delta_{\mathrm{S}}(t)$ is larger than its variation over time, implying that $\Delta_{\mathrm{S}}(t)$ may equal to $\theta_{\mathrm{B}}$ with $\theta_{\mathrm{A}} \approx 0^{\circ}$.

\subsection{Discussion}

The observed radio emission of pulsar A and its pulse profiles have remained stable and show no changes over several years since its discovery. Our results are in agreement with previous analyses that the most plausible reason for this stability of pulsar A's emission and pulse profiles is attributed to the geometric configuration of $\mathrm{A}$ in orbit, that is, a nearly aligned spin and orbit normal axes $\left(\theta_{\mathrm{A}} \sim 0^{\circ}\right)$.

We analyze pulse widths obtained at lower intensity levels in addition to those used in previous work. Our results are consistent with previous work. Based on the vacuum retarded PC beam model, as shown in Figure 4.6, the P1 component of the modeled pulse profile of M1 fits better to the observed P1 component, but modeled and observed P2 components are not in a good agreement. However, the base of both components are consistent each other. In contrast, the modeled P1 component of M2 is broader than the observed P1 component. This is because the P1 component of M2 generates from the south beam due to $\alpha_{\mathrm{A}}>90^{\circ}$ and therefore, that beam is large. Thus, we assume that our reference model is M1 and the estimated beam size is $\rho_{\mathrm{A}} \approx 30^{\circ}$ and the emission altitude at the edge of the beam is $10 \mathrm{R}_{\mathrm{NS}}$.

Recent Fermi detection of pulsed gamma-ray emission from pulsar A revealed that the peaks of high-energy and radio profiles are not aligned in spin phase (Guillemot et al., 2013). This implies that high- and low-energy emissions are produced at 
two different locations in the magnetosphere. Our geometric results based on M1 and M2 and Guillemot et al. (2013) support the assumption that A is nearly an orthogonal rotator. All our modeled pulse profiles are qualitatively in agreement with the observed profile, although none of them are perfect fits.

Using pulsar A's spin period, period derivative, and the observing frequency of $820 \mathrm{MHz}$ in the analytical expression derived by Kijak \& Gil (2003) (see Equation 1.18), the emission altitude is calculated to be $\sim 10 \mathrm{R}_{\mathrm{NS}}$. Empirical fits to normal pulsars show that the radio beam size is a function of the period as $\rho=5.4^{\circ} P^{-0.5}$, while the millisecond pulsars (where period $\lesssim 20 \mathrm{~ms}$ ) do not follow such a form (see Kramer et al., 1998). Assuming pulsar A follows the above relation, its beam size is calculated to be $\sim 38^{\circ}$. Therefore, our PC beam model estimated emission altitudes and radio beam size for pulsar A are consistent with those predicted by other methods. Furthermore, regardless of the spin period dependence on the beam size, our emission altitudes are consistent with the $<9 \mathrm{R}_{\mathrm{NS}}$ height calculated from the millisecond pulsar J0437-4715, with period of $5.75 \mathrm{~ms}$, using the phase-shift method (Gangadhara \& Thomas, 2006).

NS-NS binary mergers are considered to be progenitors of short gamma-ray bursts (Rezzolla et al., 2011; Chapman et al., 2009) as well as strong sources of gravitational waves (Abbott et al., 2008; Phinney, 1991). The spin configuration of the two neutron stars in NS-NS binaries is one of important initial conditions to simulate binary mergers (see Kastaun et al. (2013) for a concise review and references). Observational measurements of the full orbital configuration of NSNS binaries are useful to understand the final angular momentum of the central 
black hole and surrounding torus formed from the merger (e.g., Kastaun et al., 2013), binary evolution and/or supernova kick mechanism (e.g., Farr et al., 2011; Wongwathanarat et al., 2013), or gravitational-wave signals from the merger phase (e.g., Piro, 2012). For most of the known NS-NS binaries, it is impossible to measure the relative angle between spin or magnetic axes of the two NSs unless both stars are observed as radio pulsars such as the Double Pulsar. Ferdman et al. (2008) and Breton et al. (2008) estimated the spin orientation of the A and B pulsars with respect to the orbital angular momentum to be $\theta_{\mathrm{A}} \leq 14^{\circ}$ and $\theta_{\mathrm{B}}=130^{\circ} \pm 1^{\circ}$ at 95\% and $99.7 \%$ confidence, respectively. This is roughly consistent with our best-fit measurements. We also note that the magnetic axes of the two pulsars are neither aligned nor anti-aligned to each other. 


\section{Chapter 5}

\section{Modeling the non-recycled Fermi gamma-ray pulsar population}

This chapter presents a population analysis on the non-recycled Fermi gammaray pulsars. We use Fermi LAT pulsar detections to constrain the gamma-ray luminosity law. Then we use the best-fit luminosity law with the LAT upper limit fluxes on radio-detected pulsars to model the misalignment angle of the radio and gamma-ray beams. Finally, we use these results with the LAT measured diffuse fluxes to constrain the properties of gamma-ray pulsar population assuming that a fraction of diffuse fluxes is due to non-recycled pulsars. Then we make some predictions for the LAT detectability with its sensitivity limits.

The work in this chapter was submitted to Astrophysical Journal as

Perera B. B. P., McLaughlin M. A., Cordes J. M., Kerr M., Burnett T. H., Harding A. K., 2013 June 14, Submitted to ApJ; Modeling the non-recycled Fermi gamma-ray pulsar population

\subsection{Introduction}

The instrument EGRET on the Compton Gamma Ray Observatory detected pulsed gamma rays from six energetic rotation powered pulsars and three possible candidates (see Thompson, 2004, for a review). With the launch of the Fermi 
Gamma-ray Space Telescope in 2008, the number of gamma-ray pulsar detections has increased significantly, with 46 gamma-ray pulsar (GRP) detections reported in the First Fermi Large Area Telescope Catalog of Gamma-ray Pulsars (Abdo et al., 2010d, hereafter 1PC) and 83 GRP detections in the Second Fermi LAT Source Catalog (Nolan et al., 2012). It is therefore timely to use LAT detections to constrain the basic physics of pulsar gamma-ray emission and the relationship between gamma-ray and radio emission and begin to understand the GRP population. We note that the Second Fermi LAT Catalog of Gamma-ray Pulsars ${ }^{1}$ (The Fermi-LAT collaboration, 2013), which reports 117 GRP detections, became public only recently, so that we were not able to include the results in this analysis.

As mentioned in Section 1.2.2, the gamma-ray luminosity $L_{\gamma}$ must be less than the spin-down luminosity (i.e. $L_{\gamma}<\dot{E}$ ) and, equivalently, the gamma-ray efficiency $\eta=L_{\gamma} / \dot{E}<1$. Thus, we might expect the gamma-ray luminosity for pulsars to scale as $L_{\gamma} \propto \dot{E}$ or $L_{\gamma} \propto \Delta V_{\mathrm{PC}} \propto \sqrt{\dot{E}}$, where $\Delta V_{\mathrm{PC}}$ is the voltage drop across the polar cap (PC) region (see Ruderman \& Sutherland, 1975). The luminosities of radio pulsars seem to scale roughly with $\sqrt{\dot{E}}$ (Arzoumanian et al., 2002; Faucher-Giguère \& Kaspi, 2006).

The main purpose of this chapter is to determine how the observed luminosities of GRPs depend on spin-down properties. McLaughlin \& Cordes (2000) - hereafter MC00 - used pulsar detection, upper limit, and diffuse background measurements from EGRET (energies $>100 \mathrm{MeV}$ ) in a Bayesian likelihood analysis to constrain the luminosity law for GRPs and model the GRP population. Their best-fit lumi-

\footnotetext{
${ }^{1}$ http://fermi.gsfc.nasa.gov/ssc/data/access/lat/2nd_PSR_catalog/
} 
nosity law was $L_{\gamma} \propto P^{-1} \dot{P}^{0.8}$ (see Table 5.2 for parameter uncertainties). In their paper, the law was written in terms of the surface dipole magnetic field $B_{12}$, where $B_{12}=\sqrt{10^{15} P \dot{P}} \mathrm{G}$ (i.e., Equation 1.6). This law is similar to but inconsistent with $L_{\gamma} \propto \sqrt{\dot{E}} \propto P^{-1.5} \dot{P}^{0.5}$. McLaughlin \& Cordes (2003) - hereafter MC03 - updated the model used in MC00 with distances from the electron density model of Cordes \& Lazio (2002), resulting in a slightly different $\left(L_{\gamma} \propto P^{-1.1} \dot{P}^{0.6}\right)$ best-fit law. Because of the large number of GRP detections reported with Fermi, we can improve the luminosity law and also constrain other properties of GRPs, such as the beaming fraction and the degree of misalignment of the radio and gamma-ray beams. We constrain the luminosity law only from fluxes of pulsar detections, instead of using both detections and upper limits as in MC00 and MC03. That is because the large number of upper limit measurements biases and dominates the likelihood analysis, resulting in lower model-estimated luminosities for detections.

In addition to the detected fluxes of pulsars, the luminosity law depends on pulsar distance and beaming solid angle $\left(\Omega_{\gamma}\right)$, or the solid angle swept out by the gamma-ray beam. We are able to use the estimated distances of pulsars using various methods, albeit with large errors. We use a Gaussian distribution for pulsar distance uncertainties. Since we use the DM-derived distances for most of the pulsars in the analysis and they have larger uncertainties, this assumption does not affect our likelihood results. Both MC00 and MC03 assumed a beaming solid angle of $2 \pi$. In this chapter, in addition to using a constant beaming solid angle, we model the beaming solid angle individually for each pulsar according to its spin properties and observed pulse properties. We further investigate the beaming solid angle with 
geometry estimated from radio polarization (see Section 1.4 for a review of radio polarization), if available, and also with different geometrical emission models such as OG and TPC (see Section 1.3.2 for a description of these models).

We then use the constrained luminosity law in two analyses. First, we calculate gamma-ray upper limits for radio-detected pulsars and assume that their gamma-ray beam is out of our line of sight if the model-estimated flux from the luminosity law is much larger than the calculated upper limit (Section 5.6). This idea is consistent with the suggestion of Romani et al. (2011) that sub-luminous gamma-ray pulsars have gamma-ray radiation that is beamed away from our line of sight. We then use this information to model the misalignment of the radio and gamma-ray beams and then estimate the average gamma-ray beaming solid angle. Secondly in Section 5.7, we use our best-fit luminosity law in the GRP population model with measured Fermi diffuse fluxes to constrain some properties of the population such as braking index $n$, magnetic field $B$, and initial spin period $P_{0}$. In the population analysis of MC00, a single value for the magnetic field and the initial spin period of the population were used. However, as in MC03 and other studies (Faucher-Giguère \& Kaspi, 2006), we use a log-normal distribution for the surface magnetic field. We use a flat distribution for initial spin periods since the neutron star initial spin period distribution is not clearly understood (for example, see Lorimer et al., 1993). 


\subsection{Data}

\subsubsection{Pulsed gamma-ray detections from GRPs}

We use the pulsed gamma-ray detections from non-recycled pulsars reported in the 1PC and several other detections since then. The recycled MSPs have a different spatial distribution compared to that of young non-recycled pulsars. Further, their smaller magnetospheres may lead to different emission processes and luminosity laws. Thus, we exclude MSPs from our analysis and will present their results in a future work. All the detections we use in this analysis are given in Table 5.1.

The distance to each pulsar is required by our analysis. From the 38 nonrecycled detections in the $1 \mathrm{PC}, 22$ are detected at radio frequencies and we therefore estimate their distances using DMs coupled with the NE2001 electron density model (Cordes \& Lazio, 2002) as described in Section 1.2.6.3, with the following exceptions. Note that we ignored PSR J0659+1414 in the analysis due to its extremely low gamma-ray efficiency (Weltevrede et al., 2010; Takata et al., 2011b), which resulted in it being an extreme outlier in our analysis. We use the parallax-estimated distance (see Section 1.2.6.1) for PSR J0835-4510 (Dodson et al., 2003) and the 1PC reported distance for PSR J0248+6021 due to its unreliable DM-derived distance. For PSR J2021+3651, the DM-derived distance is $12 \mathrm{kpc}$. As argued in Atwood et al. (2009), such a large distance implies an unphysically high gamma-ray efficiency for some beaming models. Therefore, we use the distance that they derived, $2-4 \mathrm{kpc}$, from the pulsar wind nebula properties of the X-ray observations. Furthermore, the DM-derived distance for PSR J0742-2822 is $2.1 \mathrm{kpc}$. However, due to excess 
Table 5.1: The gamma-ray detections used in the analysis. The second and third columns are radio timing-derived period and period derivative, respectively. The fourth column is the energy flux for $E>100 \mathrm{MeV}$. The fifth column gives the distance estimate used, and the last column is the corresponding reference. Note that for most of the pulsars, we use the DM-derived distance from the NE2001 model (Cordes \& Lazio, 2002).

\begin{tabular}{|c|c|c|c|c|c|}
\hline PSR & $\begin{array}{c}\mathrm{P} \\
(\mathrm{ms})\end{array}$ & $\begin{array}{c}\dot{P} \\
\left(10^{-15} \mathrm{~s} / \mathrm{s}\right)\end{array}$ & $\begin{array}{c}\text { Energy Flux }(\mathrm{G}) \\
\left(10^{-11} \mathrm{erg} \mathrm{cm}^{-2} \mathrm{~s}^{-1}\right)\end{array}$ & $\begin{array}{l}\text { Distance } \\
\quad(\mathrm{kpc})\end{array}$ & Ref. \\
\hline $\mathrm{J} 0007+7303$ & 316.0 & 361.0 & $38.20 \pm 1.30$ & $1.40 \pm 0.30$ & (1) \\
\hline J0106+4855 & 83.2 & 0.4 & $1.93 \pm 0.18$ & $3.09 \pm 0.93$ & (2) \\
\hline J0205+6449 & 65.7 & 194.0 & $6.64 \pm 0.65$ & $4.50 \pm 1.35$ & (3) \\
\hline J0248+6021 & 217.0 & 55.1 & $3.07 \pm 0.70$ & $2-9$ & (1) \\
\hline J0534+2200 & 33.1 & 423.0 & $130.60 \pm 3.40$ & $1.73 \pm 0.52$ & (3) \\
\hline J0631+1036 & 288.0 & 105.0 & $3.04 \pm 0.61$ & $3.63 \pm 1.09$ & (3) \\
\hline J0633+1746 & 237.0 & 11.0 & $338.10 \pm 3.50$ & $0.250_{-0.062}^{+0.120}$ & (4) \\
\hline J0742-2822 & 167.0 & 16.8 & $1.82 \pm 0.42$ & $2-7$ & (5) \\
\hline J0835-4510 & 89.3 & 124.0 & $879.40 \pm 5.40$ & $0.287_{-0.017}^{+0.019}$ & (6) \\
\hline J1028-5819 & 91.4 & 16.1 & $17.70 \pm 1.40$ & $2.33 \pm 0.70$ & (3) \\
\hline J1048-5832 & 124.0 & 96.3 & $17.20 \pm 1.30$ & $2.71 \pm 0.81$ & (3) \\
\hline J1057-5226 & 197.0 & 5.8 & $27.20 \pm 0.98$ & $0.72 \pm 0.20$ & (3) \\
\hline J1119-6127 & 408.7 & 4027.8 & $7.10 \pm 0.50$ & $8.40 \pm 0.40$ & (7) \\
\hline J1124-5916 & 135.0 & 747.0 & $3.79 \pm 0.70$ & $5.70 \pm 1.71$ & (3) \\
\hline J1357-6429 & 166.2 & 357.2 & $3.39 \pm 0.33$ & $2.50 \pm 0.75$ & (3) \\
\hline J1418-6058 & 111.0 & 170.0 & $23.50 \pm 3.80$ & $2-5$ & (1) \\
\hline J1420-6048 & 68.2 & 83.2 & $15.80 \pm 3.50$ & $5.60 \pm 1.70$ & (3) \\
\hline J1509-5850 & 88.9 & 9.2 & $9.70 \pm 1.20$ & $2.60 \pm 0.80$ & (3) \\
\hline J1709-4429 & 102.0 & 93.0 & $124.00 \pm 2.60$ & $2.30 \pm 0.69$ & (3) \\
\hline J1718-3825 & 74.7 & 13.2 & $6.70 \pm 1.90$ & $3.82 \pm 1.15$ & (3) \\
\hline J1732-3131 & 196.5 & 28.0 & $24.20 \pm 1.40$ & $0.61 \pm 0.18$ & (3) \\
\hline J1741-2054 & 414.0 & 16.9 & $12.80 \pm 0.80$ & $0.38 \pm 0.11$ & (3) \\
\hline J1747-2958 & 98.8 & 61.3 & $13.10 \pm 1.70$ & $2.00 \pm 0.60$ & (3) \\
\hline J1809-2332 & 147.0 & 34.4 & $41.30 \pm 1.60$ & $1.70 \pm 1.00$ & (1) \\
\hline J1833-1034 & 61.9 & 202.0 & $10.10 \pm 1.40$ & $3.30 \pm 0.99$ & (3) \\
\hline $\mathrm{J} 1836+5925$ & 173.3 & 1.5 & $59.90 \pm 1.30$ & $0.50 \pm 0.15$ & (3) \\
\hline $\mathrm{J} 1907+0602$ & 106.6 & 86.7 & $25.40 \pm 0.60$ & $3.21 \pm 0.96$ & (3) \\
\hline J1952+3252 & 39.5 & 5.8 & $13.40 \pm 0.90$ & $3.14 \pm 0.94$ & (3) \\
\hline $\mathrm{J} 2021+3651$ & 104.0 & 95.6 & $47.00 \pm 1.80$ & $2-4$ & (8) \\
\hline $\mathrm{J} 2021+4026$ & 256.0 & 54.8 & $97.60 \pm 2.00$ & $1.50 \pm 0.45$ & (3) \\
\hline $\mathrm{J} 2030+3641$ & 200.1 & 6.5 & $3.14 \pm 0.33$ & $2-4$ & (9) \\
\hline $\mathrm{J} 2032+4127$ & 143.0 & 19.6 & $11.10 \pm 1.40$ & $3.60 \pm 1.08$ & (3) \\
\hline $\mathrm{J} 2043+2740$ & 96.1 & 1.3 & $1.55 \pm 0.32$ & $1.80 \pm 0.54$ & (3) \\
\hline $\mathrm{J} 2229+6114$ & 51.6 & 78.3 & $22.00 \pm 1.00$ & $7.50 \pm 2.25$ & (3) \\
\hline $\mathrm{J} 2240+5832$ & 139.9 & 15.2 & $1.08 \pm 0.32$ & $10.18 \pm 3.05$ & (3) \\
\hline
\end{tabular}

Note. - References: (1) 1PC - Abdo et al. (2010d); (2) Pletsch et al. (2012); (3) NE2001 Cordes \& Lazio (2002); (4) Faherty et al. (2007); (5) Weltevrede et al. (2010); (6) Dodson et al. (2003); (7) Parent et al. (2011); (8) Ałty5ood et al. (2009); (9) Camilo et al. (2012) 
density from the Gum Nebula, we use the HI measurement kinematic distance (see Section 1.2.6.2) to the pulsar, 2-7 kpc, as discussed in Weltevrede et al. (2010). In addition to these radio-loud pulsars, we adopt the distances reported in the $1 \mathrm{PC}$ (see Table 5 therein) for radio-quiet PSRs J0633+1746, J0007+7303, J1809-2332, J2021+4026, and J1418-6058. Note that PSR J0633+1746 has a parallax-estimated distance (Faherty et al., 2007). Therefore, 27 (i.e., 22 radio-loud and 5 radio-quiet pulsars) out of 38 non-recycled pulsar detections reported in the 1PC have distance estimates.

Several new pulsed gamma-ray detections with distance estimates have been reported since the $1 \mathrm{PC}$ was published. These include PSRs J0106+4855 (Pletsch et al., 2012), J1119-6127 (Parent et al., 2011), J1357-6429 (Lemoine-Goumard et al., 2011), J2030+3641 (Camilo et al., 2012), and J2240+5832 (Theureau et al., 2011). Further, we make use of updated distance estimates for three pulsars; PSRs J1732-3131, J1907+0602, and J1836+5925 (Abdo et al., 2010c; Ray et al., 2011). The DM-derived distance to PSR J1119-6127 is 17 kpc, which places the pulsar beyond the Sagittarius arm. Therefore, we use the distance of $8.4 \mathrm{kpc}$ derived from HI absorption towards the supernova remnant (Caswell et al., 2004). PSR J2030+3641 has a DM-derived distance of $8 \mathrm{kpc}$ that is beyond the Cygnus region, which is known to have excess ionized gas that contributes to the DM and thus perturbs the NE2001 distance estimate. We use the most likely distance range of 2-4 kpc as mentioned in Camilo et al. (2012). Thus, in total, there are 35 GRP detections in our sample; 27 from the 1PC and eight additional detections. For all DM-derived distances, we assumed an uncertainty of $30 \%$. 
The pulsed gamma-ray energy fluxes for the above 35 GRPs are taken from the 1PC and the above mentioned papers. All these fluxes were obtained by fitting an exponential-cutoff power-law model to the pulsar spectra with in the energy range of $100 \mathrm{MeV}<\mathrm{E}<100 \mathrm{GeV}$. The measured energy fluxes of these 35 pulsars range from $(1.1-879) \times 10^{-11} \mathrm{erg} \mathrm{s}^{-1} \mathrm{~cm}^{-2}$.

\subsubsection{Gamma-ray flux upper limits}

- The calculation of the Fermi upper limit fluxes for radio-detected pulsars was done by Dr. M. Kerr (Stanford University)-

In order to model the misalignment of radio and gamma-ray beams of radiodetected pulsars, we compute their gamma-ray upper limits using three years of Pass 7 LAT data, accepting events with zenith angles $<100^{\circ}$ and with energies between $100 \mathrm{MeV}$ and $1 \mathrm{TeV}$. The data are binned in energy (logarithmically, four per decade) and spatially using HEALPix ${ }^{2}$ (Górski et al., 2005), and likelihood analysis is performed with pointlike (Kerr, 2010), which has been shown to yield results consistent with the publicly-released LAT Science Tools analysis package. The gamma-ray background is modeled using the same diffuse models ${ }^{3}$ as the second Fermi source catalog (2FGL; Nolan et al., 2012) and an internal list of point sources based on the three-year data set. To compute the upper limit, we tessellate the sky into HEALPixels with nside $=512$ (resolution $\sim 1.6$ arcmin) and test for the

\footnotetext{
${ }^{2}$ http://healpix.jpl.nasa.gov

${ }^{3}$ http://fermi.gsfc.nasa.gov/ssc/data/access/lat/BackgroundModels.html
} 
presence of a point source in each of these pixels. We assume a representative pulsar spectral shape, a power law with index 1.8 and an exponential cutoff with cutoff energy $2 \mathrm{GeV}$. We vary the spectral normalization until the likelihood decreases to the $95 \%$ confidence level and thus determine the flux upper limit at the given position. Following this procedure, we calculate upper limits for 1496 non-recycled radio pulsars given in the ATNF pulsar catalog 4 and include them in our analysis. We note that these upper limits are not entirely compatible with the point source fluxes reported in 1FGL (Abdo et al., 2010a), which use an earlier version (Pass 6) of the LAT data and from which the luminosity law parameters are derived. However, because errors are dominated by uncertainty in distance, we do not expect these small differences to substantially affect results derived with the upper limits.

\subsubsection{Gamma-ray diffuse flux measurements}

We compute LAT diffuse fluxes to model the Galactic GRP population assuming that a fraction of diffuse flux is due to pulsars. We use the model of diffuse gamma-ray emission based on diffuse-class events recorded within the first eleven months of Pass 6 LAT data ${ }^{5}$ (Note that we used Pass 6 data because Pass 7 data were not available when this diffuse analysis was done). The file gll_iem_v02.fit contains the Galactic diffuse intensities as a function of Galactic latitude, longitude, and energy after subtracting the contribution of point sources. There are 30 logarithmically spaced energy bins between $50 \mathrm{MeV}$ and $100 \mathrm{GeV}$ in the data file

\footnotetext{
${ }^{4}$ http://www.atnf.csiro.au/people/pulsar/psrcat/

${ }^{5}$ http://fermi.gsfc.nasa.gov/ssc/data/access/lat/BackgroundModels.html
} 
(Ackermann et al., 2012). We use the given Galactic diffuse emission differential intensity (photons $\mathrm{s}^{-1} \mathrm{sr}^{-1} \mathrm{~cm}^{-2} \mathrm{Mev}^{-1}$ ) to calculate the diffuse fluxes (erg $\mathrm{s}^{-1}$ $\mathrm{sr}^{-1} \mathrm{kpc}^{-2}$ ). For our population study, we calculate the diffuse fluxes in four directions along the Galactic plane $\left(l=0^{\circ}, 20^{\circ}, 40^{\circ}\right.$, and $\left.60^{\circ}\right)$. Because the diffuse flux background models and pulsar spatial distribution are associated with large uncertainties, this coarse binning is sufficient for our work. We assume $25 \%$ uncertainties, keeping in mind that the uncertainties associated with our assumption of the pulsar contribution to the diffuse flux are much greater.

\subsection{Luminosity law}

The spin-down luminosity is $\dot{E}=4 \pi^{2} I P^{-3} \dot{P}$, where $I=10^{45} \mathrm{~g} \mathrm{~cm}^{2}$ is the typical value used for the moment of inertia, assuming a uniform sphere with a radius of $10 \mathrm{~km}$ and a mass of $1.4 \mathrm{M}_{\odot}$ (see Section 1.2 for more details). Therefore, the gamma-ray luminosity can be written as a function of $P$ and $\dot{P}$. As discussed in Section 5.1 and given in Table 4.2, pulsar luminosities scale roughly with $\sqrt{\dot{E}}$. However, previous studies such as MC00, MC03, and Arzoumanian et al. (2002) showed that the luminosity does not exactly follow the typical forms (see Table 5.2). To allow flexibility in our model and to be consistent with previous results, we parametrize the gamma-ray luminosity as

$$
L_{\gamma}=c P^{-a} \dot{P}_{15}^{b} \operatorname{erg~s}{ }^{-1}
$$

where $a, b, c$ are constants and $\dot{P}_{15}=10^{15} \dot{P}$. 
For a pulsar energy flux $G$, we write the luminosity as $L_{\gamma}=4 \pi f_{\Omega} D^{2} G$, where $f_{\Omega}$ is the beaming factor and $D$ is the distance to the pulsar. The beaming factor is a geometrical term that is used to convert the energy flux to luminosity (Watters et al., 2009). In earlier work, $f_{\Omega}$ was taken to be $1 / 4 \pi$ for a narrow gamma-ray emission beam of 1 sr (Thompson et al., 1994), or $f_{\Omega}=0.5$ for MSPs (Fierro et al., 1995). However, more recent theoretical work based on outer magnetosphere models shows that the gamma-ray emission beam sweeps nearly the entire celestial sphere, resulting in $f_{\Omega} \sim 1$ (Watters et al., 2009). On the other hand, Takata et al. (2011a) argued that most of the emission in the $\mathrm{OG}$ model is within $90^{\circ} \pm 35^{\circ}$ of the rotation axis, indicating $f_{\Omega} \sim 35^{\circ} / 90^{\circ} \sim 0.4$. In contrast, most radio pulsars have narrow $\mathrm{PC}$ radio emission beams with a best-fit half-opening angle of $\rho_{\mathrm{r}}=5.8^{\circ} P^{-0.5}$ (Rankin, 1993a), where $P$ is in seconds, implying $f_{\Omega} \sim 0.1$ for an orthogonal rotator with spin period of 1 second.

\subsection{Luminosity law likelihood analysis}

In this section we describe how we use detections to calculate the best-fit luminosity law. First we calculate the luminosity of a detected pulsar $L_{\gamma}(\Theta)$ from Equation (5.1) based on $P$ and $\dot{P}$ for a given model parameter combination $a, b$, and $c$ denoted as $\Theta$. Then we calculate the model-estimated energy flux $G_{\text {mod }}(\Theta, D)=$ $L_{\gamma}(\Theta) / 4 \pi f_{\Omega} D^{2}$ for the estimated pulsar distance. The beaming factor $f_{\Omega}$ is modeled through different methods (see Section 5.5). Then we calculate the likelihood of this model-estimated flux based on the pulsar's measured gamma-ray flux $(G)$. We write 
the individual likelihood $\mathcal{L}_{\text {det,i }}(\Theta)$ for a given model $\Theta$ for a given GRP $i$ as

$$
\mathcal{L}_{\text {det, } \mathrm{i}}(\Theta)=\int_{D_{\mathrm{l}}}^{D_{\mathrm{u}}} d D f(D) g(\Theta, D)
$$

where $g(\Theta, D)=\left(2 \pi \sigma^{2}\right)^{-1 / 2} \exp \left(-\left(G_{\text {mod }}(\Theta, D)-G\right) / 2 \sigma^{2}\right)$ and $f(D)=\left(2 \pi d^{2}\right)^{-1 / 2} \exp (-(D-$ $\left.D_{0}\right) / 2 d^{2}$. The quantity $\sigma$ is the error on the measured energy flux. In the distance term, $D_{0}$ is the best estimated distance, $D_{\mathrm{l}}$ and $D_{\mathrm{u}}$ are lower and upper estimates, respectively, and $d$ is the error. Therefore, the maximum likelihood occurs when the model-estimated flux is equal to the measured flux at the best distance estimate. Then we calculate the total likelihood from all GRPs for a given model $\Theta$ as

$$
\mathcal{L}_{\operatorname{det}}(\Theta)=\prod_{\mathrm{i}=1}^{N_{\mathrm{det}}} \mathcal{L}_{\mathrm{det}, \mathrm{i}}(\Theta)
$$

where $N_{\text {det }}$ is the total number of detections.

We assume that the maximum possible luminosity of a pulsar is some fraction of the spin-down luminosity, i.e. $L_{\max }=\epsilon_{\gamma} \dot{E}$. We assume $\epsilon_{\gamma}=1$, in contrast to MC00's assumption of $\epsilon_{\gamma}=0.5$. This is consistent with the highest realistic $\epsilon_{\gamma}$ for a pulsar detection assuming $f_{\Omega}=1$ as in $1 \mathrm{PC}\left(\epsilon_{\gamma} \approx 0.8\right.$ for PSR J0633 +1746$)$. Note that as reported in the same study, PSR J2021 + 4026 has $\epsilon_{\gamma} \approx 2.2$ with $f_{\Omega}=1$, indicating that $f_{\Omega}$ for this pulsar is less than unity or the distance estimate is incorrect. If the model-estimated luminosity is greater than $\epsilon_{\gamma} \dot{E}$, we assign a likelihood of zero to that particular parameter combination for that pulsar. In contrast, if the model predicted luminosity was greater than $\epsilon_{\gamma} \dot{E}, \mathrm{MCO0}$ set the maximum luminosity equal to $\epsilon_{\gamma} \dot{E}$. Our modified method ensures that unrealistic 
models do not contribute to the likelihood.

We use a grid search to determine the best-fit values for the three model parameters. For each combination of parameters, we calculate the likelihood for detections and then determine the marginalized probability density functions (PDFs) as

$$
f\left(\Theta_{\mathrm{j}}\right)=\frac{\int_{\mathrm{i} \neq \mathrm{j}} d \Theta_{\mathrm{i}} \mathcal{L}_{\mathrm{det}}\left(\Theta_{\mathrm{i}}\right)}{\int d \Theta_{\mathrm{i}} \mathcal{L}_{\mathrm{det}}\left(\Theta_{\mathrm{i}}\right)},
$$

where the numerator gives the total likelihood for any given parameter $\Theta_{j}$ across each grid cell. The peak of this PDF gives the best-fit value of the parameter and then the error can be calculated for a desired confidence level.

\subsection{Luminosity law analysis and results}

We model the beaming factor using several different methods. MC00 assumed that the beaming solid angle $\Omega_{\gamma}$ of the gamma-ray emission is $2 \pi$, or the beaming factor $f_{\Omega}=0.5$, meaning the gamma-ray emission covers half of the sky. If the emission is uniform across the instantaneous gamma-ray beam (i.e. the solid angle of the gamma-ray beam itself), then the maximum possible $f_{\Omega}$ would be unity, depending on the magnetic inclination and the angular radius of the beam. Note that the instantaneous gamma-ray beam solid angle $\left(\Omega_{\gamma \mathrm{i}}\right)$ is smaller than the beaming solid angle $\Omega_{\gamma}$. However, if the emission is not uniform across the instantaneous gamma-ray beam and the true average flux across the beam itself is greater than what we have measured, then $f_{\Omega}$ can have values greater than unity. Watters et al. 
(2009) showed that for a given outer magnetosphere emission model, $f_{\Omega}$ can have a wide range of values depending on the geometry of the pulsar and the characteristic gap width.

As the first step, we model the beaming factor as a constant (Section 5.5.1), keeping in mind that it is likely dependent on the emission geometry and the other pulsar properties. In later sections, we use more sophisticated models for $f_{\Omega}$. In all these methods, we set a maximum value of $f_{\Omega}=1.5$ in order to be consistent with Watters et al. (2009).

\subsubsection{Constant beaming factor}

First, we use $f_{\Omega}=0.5$, which is then a similar analysis to MC00 and MC03, but using a larger sample of pulsar detections (35 compared to 7). By searching the entire parameter space for $a, b$, and $c$, we calculate the marginalized PDFs and $95 \%$ confidence intervals to find $a=1.43_{-0.04}^{+0.03}, b=0.40 \pm 0.02$, and $\log c=$ $32.53 \pm 0.02$. Comparisons with previous and expected forms are given in Table 5.2. We list reduced chi-squared values for all fits in this table. These are all significantly greater than unity, indicating that none of our simple models are perfect fits to the data and that uncertainties in distance and beaming models dominate. Therefore, the uncertainties of our best-fit parameters are likely underestimated. The new luminosity law is substantially different from that of MC00. The new parameters have smaller errors compared to the previous estimates, likely due to the larger number of flux measurements. The last column of the table gives the number of 
Table 5.2: Constrained gamma-ray luminosity laws: $L_{\gamma}=c P^{-a} \dot{P}_{15}^{b}$. The fifth column is the $\log$ of the $\chi_{\text {red }}^{2}$ value of the best-fit solution. The last column shows the number of severe non-detections out of 1496 pulsars for each model from the beaming analysis of the upper-limit pulsars.

\begin{tabular}{lccccc}
\hline & $a$ & $b$ & $\log (c)$ & $\log \left(\chi_{\text {red }}^{2}\right)$ & $N_{\bar{\gamma}}$ \\
\hline$L_{\gamma} \propto \dot{E}$ & 3 & 1 & - & - & - \\
$L_{\gamma} \propto \sqrt{\dot{E}}$ & 1.5 & 0.5 & - & - & - \\
McLaughlin \& Cordes (2000) & $1.0(1)$ & $0.8(2)$ & $32.0(2)$ & - & - \\
McLaughlin \& Cordes (2003) & 1.1 & 0.6 & 32.4 & - & - \\
Arzoumanian et al. (2002) & $1.3(3)$ & $0.4(1)$ & $29.3(1)$ & - & - \\
\hline This work & & & & & \\
\hline Schematic beam models: & & & & & \\
$f_{\Omega}=0.5$ & $1.43(4)$ & $0.40(2)$ & $32.53(2)$ & 2.94 & 114 \\
$f_{\Omega}=1$ & $1.43(4)$ & $0.40(3)$ & $32.83(2)$ & 2.93 & 114 \\
$\Omega_{\gamma}=\lambda P^{-\nu}$ & $0(1)$ & $0.5(1)$ & $33.3(3)$ & 5.1 & 94 \\
$\Omega_{\gamma}=\lambda(\Delta \phi)^{\nu}$ & $2.0(2)$ & $0.4(2)$ & $32.0(6)$ & 4.54 & $41^{\dagger}$ \\
Emission gap models: & & & & & \\
TPC & $1.45(4)$ & $0.41(2)$ & $32.81(3)$ & 2.93 & 162 \\
OG & $1.36(3)$ & $0.44(2)$ & $32.82(3)$ & 2.91 & 117 \\
PC & $1.11(4)$ & $0.38(2)$ & $32.74(1)$ & 3.49 & 162 \\
\hline
\end{tabular}

Note. - The number in parentheses is the $2 \sigma$ uncertainty in the last quoted digit. $\dagger$ Assuming a beaming factor of unity for pulsars with flux upper-limits.

severe non-detections for each model (see Section 5.6 for more discussion). A "severe non-detection" is defined as when the expected energy flux $(\hat{G})$ for an upper-limit pulsar from the best-fit luminosity law is greater than its measured upper limit flux $\left(G_{\text {up }}\right)$ by more than the $2 \sigma$ error (i.e. $\hat{G}>G_{\text {up }}+2 \sigma$ ). In order to account for the distance uncertainty, we define the expected flux as $\hat{G}=L_{\gamma} / 4 \pi f_{\Omega} D_{\mathrm{u}}^{2}$, where $D_{\mathrm{u}}$ is the distance upper limit.

We then examined the effect of using other constant values of the beaming factor. Most of the recent studies assume that $f_{\Omega}$ is unity (1PC; Theureau et al., 2011; Pletsch et al., 2012). With this assumption, the marginalized PDFs of the 

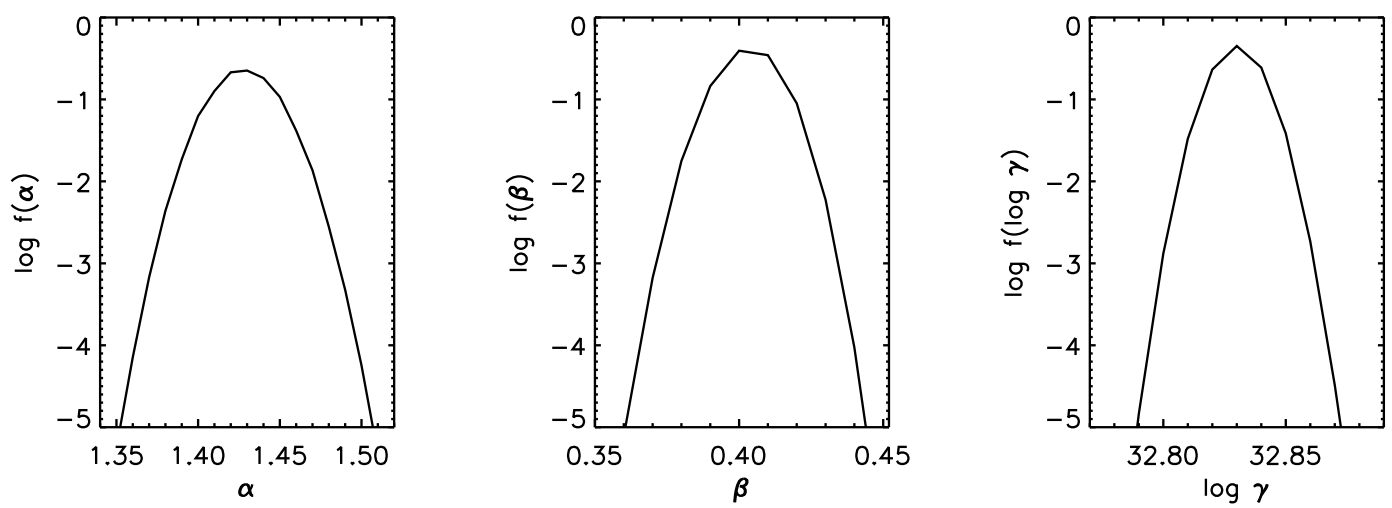

Figure 5.1: Marginalized PDFs for model parameters of the luminosity law when $f_{\Omega}=1$. The best-fit parameters at $95 \%$ confidence level are $a=1.43_{-0.04}^{+0.03}, b=$ $0.40_{-0.02}^{+0.03}$, and $\log c=32.83 \pm 0.02$.

three parameters are shown in Figure 5.1. With the 95\% confidence interval, our best-fit model parameters are $a=1.43_{-0.04}^{+0.03}, b=0.40_{-0.02}^{+0.03}$, and $\log c=32.83 \pm 0.02$ (see Table 5.2). Note that when the beaming factor is a constant, the luminosity scale $(c)$ changes slightly while the model parameters ( $a$ and $b$ ) remain nearly constant. Figure 5.2 shows the model-estimated and measured fluxes of these 35 GRPs. The errors of the model-estimated fluxes are calculated from the $2 \sigma$ errors of the three parameters and the errors on the distances. Figure 5.3 shows how the best-fit luminosities of these detections vary with their spin-down luminosities, with a luminosity law similar to $L_{\gamma} \propto \sqrt{\dot{E}}$. We assume this model as our reference model and use this particular luminosity law in the population analysis.

Note that the uncertainty in the model-estimated flux for J0835-4510 (Vela) is very small due to the well-constrained distance from parallax (Dodson et al., 2003). Therefore, Vela was weighted heavily in the search. Some of the outliers in Figure 5.2 (PSRs J2021+4026, J1709-4429, and J1119-6127) may be associated with poorly constrained distances. For example, we used the kinematic distance of $1.5 \mathrm{kpc}$ for 


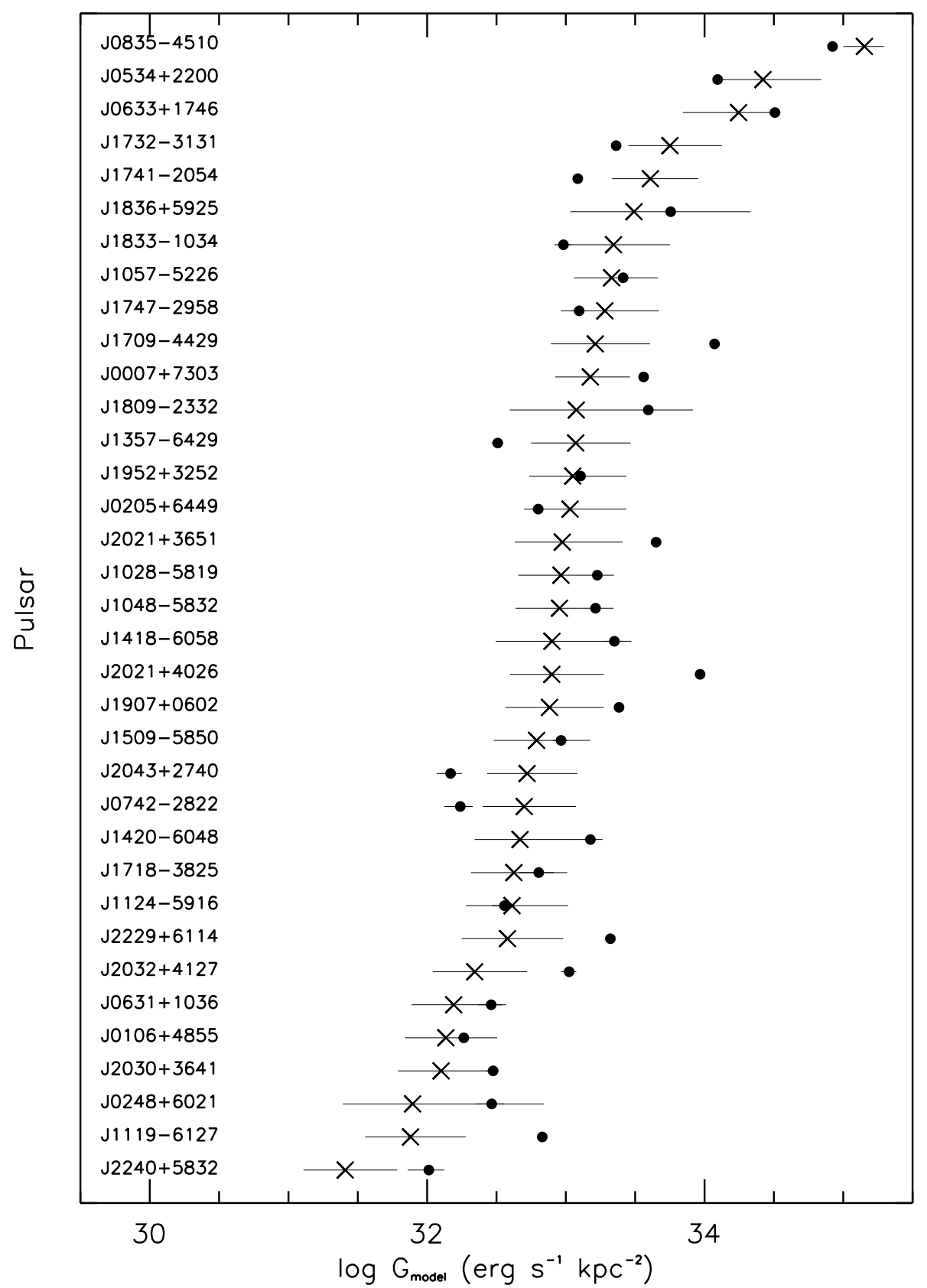

Figure 5.2: Model-estimated energy fluxes of 35 LAT-detected CPs with estimated distances for the case of a constant beaming factor, $f_{\Omega}=1$. The luminosity model that was used to calculate the model-estimated fluxes is $L_{\gamma}=10^{32.83} \mathrm{P}^{-1.43} \dot{P}^{0.40}$ (see Table 4.2). The model-estimated fluxes are marked by crosses and sorted in decreasing order. Dots represent the measured fluxes from the LAT. The errors of the model-estimated fluxes are determined through $2 \sigma$ errors of the three parameters $a, b$, and $c$ and the uncertainty of the distance. The errors of measured energy fluxes are much smaller than of the model-estimated energy fluxes, as the latter incorporates distance uncertainties. The logarithmic value of the $\chi_{\text {red }}^{2}$ is 2.93 . Some of the outliers may be due to poorly estimated distance (see text). 


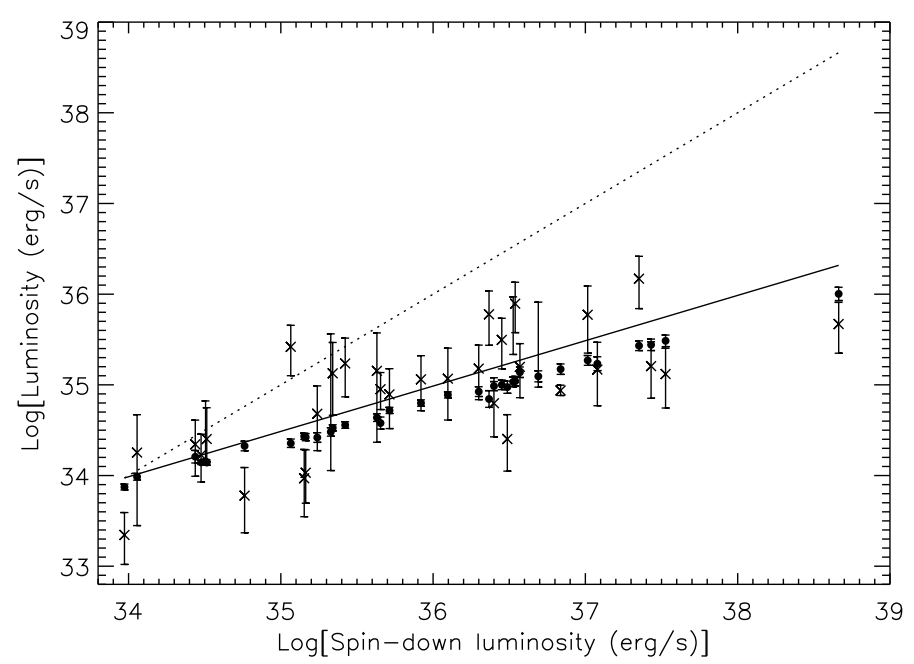

Figure 5.3: Model-estimated luminosity vs. spin-down luminosity (Circles) of the 35 LAT-detected pulsars for the case of a constant beaming factor, $f_{\Omega}=1$ (see Table 5.2 for the best-fit luminosity law). Note that the estimated errors are small due small errors on the best-fit luminosity parameters. The dotted line shows $L_{\gamma}=\dot{E}$. The solid line shows the form of $L_{\gamma} \propto \sqrt{\dot{E}}$. It is clearly seen that the model-estimated luminosities of these detections closely follow the latter form. For comparison, the computed luminosities for these pulsars based on the measured fluxes and estimated distances for $f_{\Omega}=1$ are shown (Crosses). The error of these luminosities are estimated from the uncertainties of distances and measured fluxes. 
the radio-quiet PSR J2021+4026 (Landecker et al., 1980). The thermal emission component of the recent X-ray observations suggest a relatively large distance, $\sim$ $6 \mathrm{kpc}$, at odds with a proposed association with supernova remnant G78 +2.1 (Weisskopf et al., 2011). These outliers are common for all of the methods that we follow in this paper (see Section 5.5.4, Figures 5.4 and 5.5).

\subsubsection{Period-dependent beaming factor}

Considering particle corotation, the boundary of the magnetosphere is defined with respect to the light cylinder; $R_{\mathrm{LC}}=c P / 2 \pi$. Therefore, the emission geometry and pattern may depend on the pulsar period. Empirical fits to radio pulsars show that the half-opening angle $\rho_{\mathrm{r}}$ of the radio emission beam is a function of its period, $\rho_{\mathrm{r}}=5.8^{\circ} P^{-0.5}$ (see Section 1.5 for more details). With this motivation, we model $f_{\Omega}=\lambda P^{-\nu}$, including two additional parameters $\lambda$ and $\nu$. We vary the parameter $\nu$ from -1 to 3 , allowing $f_{\Omega}$ to have a maximum value of 1.5 . Then we follow the same likelihood analysis with a grid search of all five parameters $(a, b, c, \lambda$, and $\nu)$ to find marginalized PDFs. The best-fit parameters are $a=0.1_{-0.2}^{+1.2}, b=0.50 \pm 0.08, \log c=$ $33.3_{-0.3}^{+0.1}, \lambda=2.2_{-1.1}^{+0.2}$, and $\nu=1.8_{-1.2}^{+0.2}$. The PDFs of $a, \lambda$, and $\nu$ show that these cannot be constrained to single values, reflected in the larger errors (see Table 5.2; best-fit $\left.\log \left(\chi_{\text {red }}^{2}\right)=5.1\right)$. The non-zero $\nu$ may imply that $f_{\Omega}$ could have some period dependence with a more complex form dependent on additional parameters such as magnetic inclination and period derivative. 


\subsubsection{Phase-shift dependent beaming factor}

As described in Section 1.5, the emission altitudes of radio pulsars can be determined by using the phase shifts of pulse components in their pulse profiles (Gangadhara \& Gupta, 2001; Dyks et al., 2004). Applying this method for pulsars that are both radio and gamma-ray loud, it is possible that the phase shift between the radio and the gamma-ray pulse profile peaks can be explained with a similar effect, leading to emission altitude estimates. Assuming that the high-energy emission is generated within the outer magnetosphere, the height of the emission is related to the size of the instantaneous gamma-ray beam, and hence the beaming factor. Therefore, we calculate the phase shift of the leading and trailing components of the gamma-ray profile with respect to the radio pulse profile peak and explore whether the beaming factor depends on this quantity. Since the gamma-ray emission altitudes are larger than the radio emission altitudes, our assumption that the radio emission is generated on the neutron star surface is valid.

Assuming dipolar field lines, we can express the beam size as a power law of emission height (see Section 1.5 for a detailed discussion), which is proportional to the phase shift (see Gangadhara \& Gupta, 2001). Therefore, the beaming factor can be modeled as $f_{\Omega}=\lambda \Delta \phi^{\nu} / 4 \pi$, where $\Delta \phi$ is the phase shift. We used only radio-loud GRPs that have multiple peaks in the gamma-ray pulse profile (19 out of 35 pulsar detections). We ignored the Crab pulsar $(\mathrm{J} 0534+2200)$ due to the nearly zero shift in phase between the peaks of the radio and gamma-ray pulse profiles, which implies that they arise from the same altitude. The best-fit parameters are 
$a=2.0 \pm 0.2, b=0.4 \pm 0.2, \log c=32.0_{-0.6}^{+0.4}, \lambda=5.2_{-3.3}^{+14.6}$, and $\nu=-0.4_{-0.2}^{+0.3}$. The parameter $\lambda$ is poorly constrained and its PDF shows that it has several equally significant solutions, resulting in a poor fit. Therefore, the phase-shift method is not applicable for gamma-ray and radio profiles, assuming a simple beam geometry. Including more parameters such as magnetic inclination with advanced emission geometries may lead to better fits.

\subsubsection{Outer magnetosphere model dependent beaming factor}

Outer magnetosphere models can explain the broad profiles and observed emission bridges between pulse peaks in GRPs (Watters \& Romani, 2011). In this section, we apply the commonly used outer magnetosphere OG and TPC emission models (see Section 1.3.2) in the luminosity analysis.

The emission patterns produced by outer magnetosphere and PC models depend on the geometry of the pulsar, which can be determined from two main methods. For young pulsars with bright X-ray pulsar wind nebulae (PWNe), the viewing angle $\zeta$ can be constrained by fitting the Doppler-boosted PWN torus $(\mathrm{Ng} \&$ Romani, 2008), where $\zeta$ is the angle between the line of sight and the rotation axis of the pulsar. However, since there are only a few pulsars with PWNe torii, the technique is limited. Radio polarization measurements, along with the rotating vector model (see Section 1.4), can also be used to constrain the magnetic inclination $(\alpha)$ with respect to the rotation axis and the impact parameter $(\beta)$. This model fits the S-shaped sweep of the polarization position angle of the linear polarization as a 
function of $\alpha$ and $\beta$.

Unfortunately, radio polarization has not been measured for all of the pulsars in our study. Rankin (1993b) reported radio polarization measurements and determined the geometrical angles $\alpha$ and $\beta$ for about 150 radio pulsars. Mitra \& Rankin (2011) further analyzed 50 pulsars that have asymmetric pulse profiles and polarization position angles and determined their geometry. We have calculated Fermi upper limits for 121 pulsars with known geometry from these studies. The geometry has been derived for only 14 of the LAT-detected pulsars with estimated distances; see Table 5.3. For these 14 pulsars, we calculate $f_{\Omega}$ for TPC and OG models using the analytical expressions derived in Watters et al. (2009) that model the beaming factor as a function of the pulsar geometry, $\alpha$ and $\zeta(=\beta+\alpha)$, and the characteristic fractional gap width $w$. Watters et al. (2009) obtained $w$ from the assumption that $w \propto \eta=L_{\gamma} / \dot{E}$ with the luminosity form of $L_{\gamma} \propto \sqrt{\dot{E}}$. However, for $f_{\Omega}$ to be independent of the luminosity law, we assume that $w \approx 0$, implying the gamma-ray emission is generated along the last closed field lines, which is consistent with original OG (e.g. Chiang \& Romani, 1992, 1994) and TPC (Dyks \& Rudak, 2003) models. Therefore, we estimate $f_{\Omega}$ for all the 14 pulsars according to their geometries using expressions given in Watters et al. (2009) with $w \approx 0$. For the rest of the 21 geometry-unknown detections, we use $f_{\Omega}=1$.

For the TPC model, we use Equation 7 and 8 of Watters et al. (2009) to calculate $f_{\Omega}$ for the two cases $\zeta>\zeta_{\text {I }}$ and $\zeta<\zeta_{\text {I }}$ with $w=0.001$, where $\zeta_{\text {I }}=$ $(75+100 w)-(60+1 / w)(\alpha / 90)^{2(1-w)}$ is Equation 6 of the same study. By searching over the three model parameters, we calculated the best-fit values to be $a=1.45_{-0.03}^{+0.04}$, 
Table 5.3: Geometry estimated from radio polarization for Fermi-detected pulsars; the magnetic inclination $\alpha$, the impact parameter $\beta$, and half-opening angle of the radio beam $\rho_{\text {r }}$ are listed.

\begin{tabular}{ccccc}
\hline Pulsar & $\alpha^{\dagger \dagger}$ & $\beta^{\dagger \dagger}$ & $\rho_{\mathrm{r}}$ & Ref \\
\hline $\mathrm{J} 0248+6021$ & $40^{\circ}-80^{\circ}$ & $+5^{\circ}$ & $5^{\circ \dagger}$ & $(1)$ \\
$\mathrm{J} 0631+1036$ & $90^{\circ}$ & $-4^{\circ}$ & $18^{\circ}$ & $(2)$ \\
$\mathrm{J} 0742-2822$ & $80^{\circ}-110^{\circ}$ & $-7^{\circ}$ & $18^{\circ}$ & $(2)$ \\
$\mathrm{J} 0835-4510$ & $-137^{\circ}$ & $6.5^{\circ}$ & $6.5^{\circ \dagger}$ & $(3)$ \\
$\mathrm{J} 1057-5226$ & $75^{\circ}$ & $-6^{\circ}$ & $6^{\circ \dagger}$ & $(4),(5)$ \\
$\mathrm{J} 1119-6127$ & $20^{\circ}-30^{\circ}$ & $-30^{\circ}-0^{\circ}$ & $14^{\circ}$ & $(6),(7)$ \\
$\mathrm{J} 1420-6048$ & $20^{\circ}$ & $-0.5^{\circ}$ & $15^{\circ}$ & $(2),(8)$ \\
$\mathrm{J} 1709-4429$ & $36^{\circ}$ & $17^{\circ}$ & $17^{\circ \dagger}$ & $(5),(9)$ \\
$\mathrm{J} 1718-3825$ & $20^{\circ}$ & $4^{\circ}$ & $13^{\circ}$ & $(2)$ \\
$\mathrm{J} 2021+3651$ & $70^{\circ}$ & $15^{\circ}$ & $15^{\circ \dagger}$ & $(10)$ \\
$\mathrm{J} 2030+3641$ & $20^{\circ}-90^{\circ}$ & $20^{\circ}-80^{\circ}$ & $5^{\circ \dagger}$ & $(11)$ \\
$\mathrm{J} 2043+2740$ & $52^{\circ}-83^{\circ}$ & $60^{\circ}-88^{\circ}$ & $8^{\circ \dagger}$ & $(12)$ \\
$\mathrm{J} 2229+6114$ & $55^{\circ}$ & $-9^{\circ}$ & $9^{\circ \dagger}$ & $(13)$ \\
$\mathrm{J} 2240+5832$ & $108^{\circ}$ & $123^{\circ}$ & $15^{\circ \dagger}$ & $(1)$ \\
\hline
\end{tabular}

Note. $-{ }^{\dagger}$ Half-opening angle $\rho_{\mathrm{r}}$ has not been constrained. Therefore, we assume $\rho_{\mathrm{r}}=|\beta|$ for any required calculation.

${ }^{\dagger \dagger}$ According to radio polarization, the two angles $\alpha$ and $\beta$ are associated with larger errors.

References: (1) Theureau et al. (2011); (2) Weltevrede et al. (2010); (3) Johnston et al. (2005); (4) Weltevrede \& Wright (2009); (5) Abdo et al. (2010b); (6) Weltevrede et al. (2011); (7) Parent et al. (2011); (8) Roberts et al. (2001); (9) Ng \& Romani (2008); (10) Atwood et al. (2009); (11) Camilo et al. (2012); (12) Noutsos et al. (2011); (13) Abdo et al. (2009) 
$b=0.41 \pm 0.02$, and $\log c=32.81_{-0.03}^{+0.02}$. The luminosity law is listed in Table 5.2 and model-estimated fluxes are shown in Figure 5.4.

Then we constrain the luminosity law according to the OG model. Again, we use the derived results of Watters et al. (2009) with $w=0.001$. The corresponding analytic expressions for $f_{\Omega}$ for the OG model are given in Equations (9) and (10) in their study for the two cases of $\zeta>60^{\circ}$ and $\zeta<60^{\circ}$. The likelihood analysis then constrained the best-fit values to be $a=1.36 \pm 0.03, b=0.44 \pm 0.02$, and $\log c=$ $32.82_{-0.02}^{+0.03}$. The model-estimated and measured fluxes are shown in Figure 5.5.

\subsection{Determining the geometry of gamma-ray and radio beams}

Gamma-ray and radio emission are likely generated in different regions of the magnetosphere for most pulsars, with gamma rays originating in the outer magnetosphere (Romani, 1996) and radio waves in the polar cap region (Ruderman \& Sutherland, 1975). In general, the $\mathrm{PC}$ radio beam is assumed to be magneticpole-centered, while the gamma-ray emission geometry is complicated due to its outer magnetosphere origin. The sky maps (i.e. emission pattern in $\zeta$ vs $\phi_{\text {spin }}$ space, where $\phi_{\text {spin }}$ is the spin longitude) of the OG and TPC models (see Bai \& Spitkovsky, 2010b) show that the gamma-ray fan-like emission is centered on the spin equator (i.e. $\zeta \approx 90^{\circ}$ ). In this section, we model the fraction of radio-detected pulsars with radio beams within their fan-like gamma-ray beams by using pulsar upper limits with our best-fit luminosity laws from different methods as described in the previous section. 


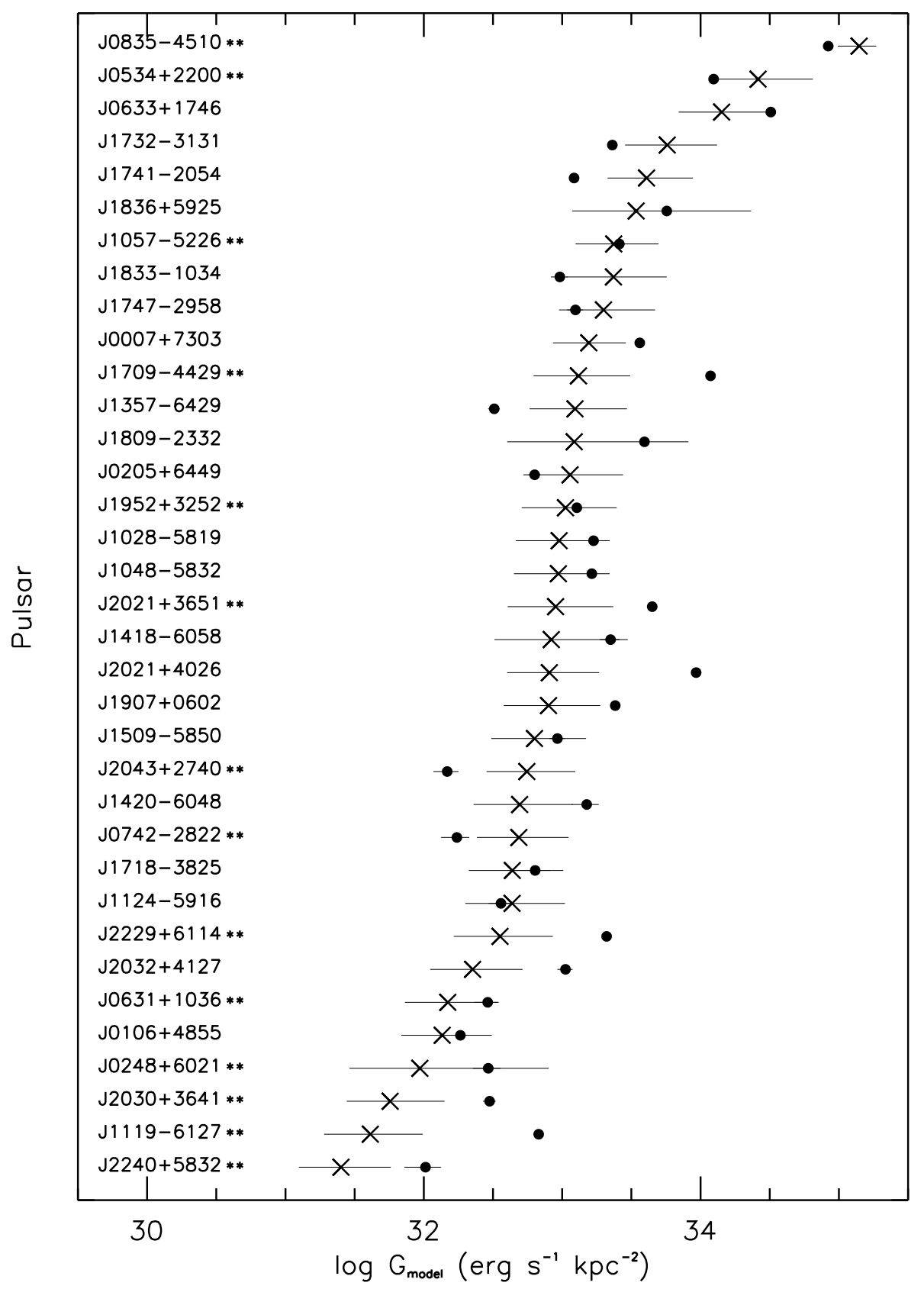

Figure 5.4: Same as Figure 5.2, but for the TPC model. The best-fit luminosity model is $L_{\gamma}=10^{32.81} P^{-1.45} \dot{P}^{0.41}$ (see Table 5.2). Note that the pulsars are marked with $(* *)$ have estimated geometry and included in the analysis. We used $f_{\Omega}=1$ for the pulsars that do not have estimated geometry. 


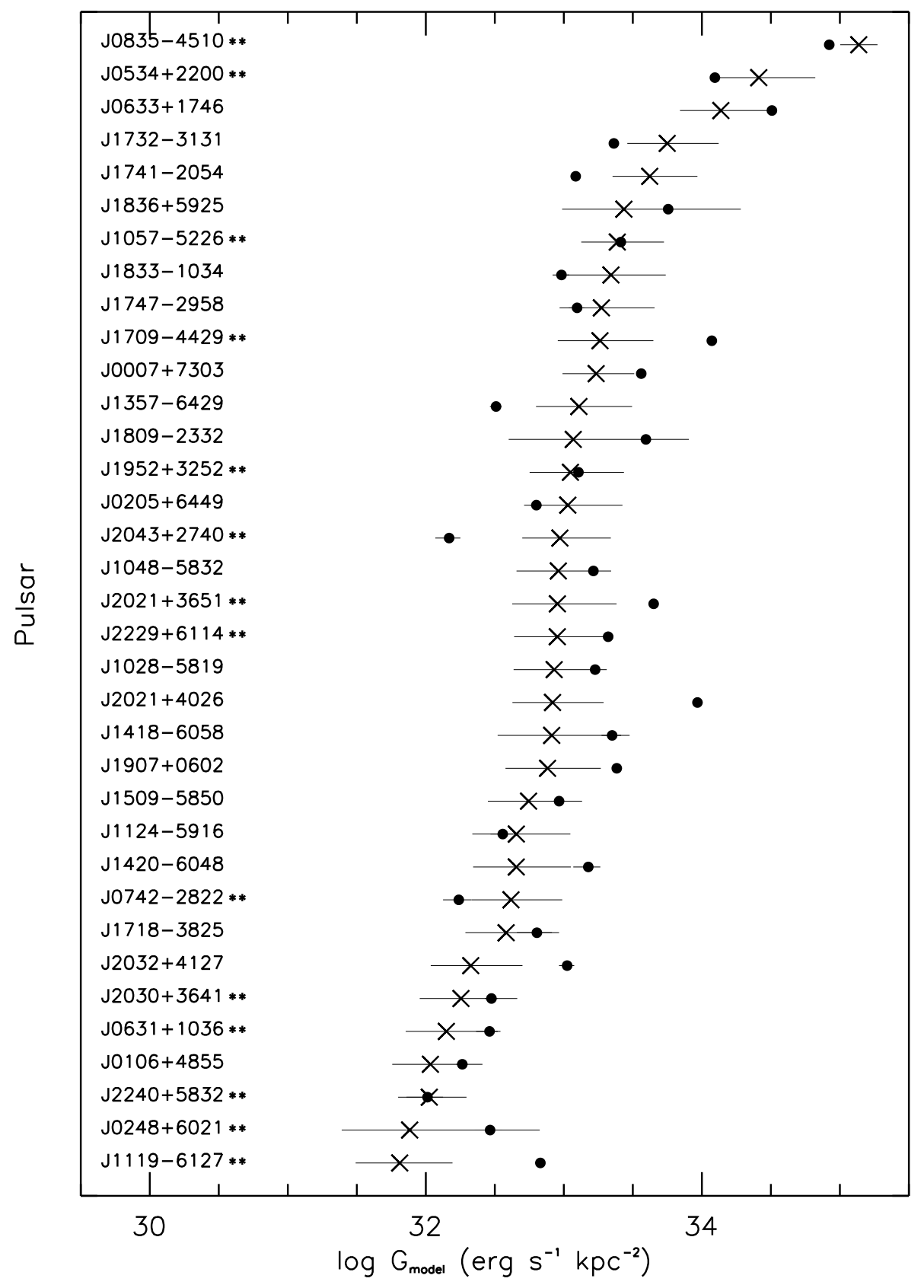

Figure 5.5: Same as Figure 5.2 and 5.4, but for the OG model. The best-fit luminosity model is $L_{\gamma}=10^{32.82} P^{-1.36} \dot{P}^{0.44}$ (see Table 5.2). 
We first calculate the number of observed severe non-detections and then calculate the expected number of severe non-detections given $\rho_{\gamma}$ (see Section 5.5.1 for the definition of a severe non-detection). The most likely explanation for a severe non-detection is that the gamma-ray beam does not intersect our line of sight due to radio and gamma-ray beam misalignment, with the radio beam outside of the gamma-ray beam. By applying the above condition to all upper-limit pulsars, we can estimate the number of observed severe non-detections $\left(N_{\bar{\gamma}}\right)$. The number of expected severe non-detections $\left(\hat{N}_{\bar{\gamma}}\right)$ can be estimated from $\rho_{\gamma}$. Then we use $\rho_{\gamma}$ to calculate the best-fit beaming solid angle $\Omega_{\gamma}$ of the gamma-ray emission.

We assume that the gamma-ray beam is centered around the spin equator and has a symmetric half-opening angle of $\rho_{\gamma}$, above and below the equator. However, the sky maps of OG and TPC models show that the gamma-ray emission pattern is strongly dependent on the magnetic inclination $\alpha$ (Bai \& Spitkovsky, 2010b). Therefore, the half-opening angle of the beam in our model is a function of $\alpha$. As shown in Pierbattista et al. (2012), the half-opening angle also depends on the age of the pulsar. Since we are interested in the average properties of the population, we do not include the age dependency on the half-opening angle in our simple model. The skew angle between the radio and gamma-ray beams is defined as $\theta_{\text {rg }}$ (i.e., a polar angle measured from the spin equator). With the assumption that $\theta_{\mathrm{rg}}$ is uniformly distributed, we write the PDF as $f_{\theta_{\mathrm{rg}}}\left(\theta_{\mathrm{rg}}\right) \propto \sin \theta_{\mathrm{rg}}$ where $0 \leq \theta_{\mathrm{rg}}<\rho_{\gamma}(\alpha)$. Assuming that the radio beam size is small compared to that of the gamma-ray beam, we define the fraction of radio-detected pulsars that have gamma-ray beams that intersect our line of sight $f_{\mathrm{r} \gamma}$ as follows. 
The beaming solid angle of the pulsar can be obtained by integrating over $\zeta$ and $\phi_{\text {spin }}$ as

$$
\Omega_{\gamma, \mathrm{i}}(\alpha)=\int_{0}^{2 \pi} d \phi_{\mathrm{spin}} \int_{\left(\pi / 2-\rho_{\gamma}(\alpha)\right)}^{\left(\pi / 2+\rho_{\gamma}(\alpha)\right)} \sin \zeta d \zeta
$$

By examining sky maps of Bai \& Spitkovsky (2010b), we find that, roughly, $\rho_{\gamma, \operatorname{TPC}}(\alpha)=$ $71^{\circ}\left(\alpha / 90^{\circ}\right)^{0.7}$ and $\rho_{\gamma, \mathrm{OG}}(\alpha)=75^{\circ}\left(\alpha / 90^{\circ}\right)^{0.85}$ for TPC and OG models, respectively. Then the beaming fraction is given by $f_{\Omega, \mathrm{i}}(\alpha) \approx \Omega_{\gamma, \mathrm{i}}(\alpha) / 4 \pi$. We write $f_{\mathrm{r} \gamma}$ as a function of $\alpha$ as

$$
f_{\mathrm{r} \gamma}(\alpha)=\frac{\sum_{\mathrm{i}=1}^{N_{\mathrm{r}}} \int_{0}^{\rho_{\gamma}(\alpha)} f_{\Omega, \mathrm{i}}(\alpha) f_{\theta_{\mathrm{rg}}}\left(\theta_{\mathrm{rg}}\right) d \theta_{\mathrm{rg}}}{\sum_{\mathrm{i}=1}^{N_{\mathrm{r}}} \int_{0}^{\rho_{\gamma}(\alpha)} f_{\theta_{\mathrm{rg}}}\left(\theta_{\mathrm{rg}}\right) d \theta_{\mathrm{rg}}}
$$

where $N_{\mathrm{r}}$ is the number of radio-detected pulsars in the sample. Then we estimate the number of expected severe non-detections for a given model from $\hat{N}_{\bar{\gamma}}=(1-$ $\left.f_{\mathrm{r} \gamma}\right) N_{\mathrm{r}}$. For a given $\alpha$, we then calculate the likelihood function based on $\hat{N}_{\bar{\gamma}}$. We define the likelihood fraction $\mathcal{L}=\exp \left(-0.5\left(N_{\bar{\gamma}}-\hat{N}_{\bar{\gamma}}\right)^{2} / N_{\bar{\gamma}}\right)$ when $\hat{N}_{\bar{\gamma}}>100$ and $\mathcal{L}=\left(\hat{N}_{\bar{\gamma}}\right)^{N_{\bar{\gamma}}} \exp \left(-\hat{N}_{\bar{\gamma}}\right) / N_{\bar{\gamma}}$ ! otherwise. We search $\alpha$ to find the best-fit value where the likelihood fraction is a maximum.

With the best-fit luminosity laws given in Section 5.5, we determine $\alpha$, leading to $\rho_{\gamma}$. For the model of $f_{\Omega}=1$, there are 114 severe non-detections (i.e. where the expected flux is much greater than the measured upper limit flux). According to the explanation of a severe non-detection given above, about $92 \%(=[1-114 / 1496] \times$ 


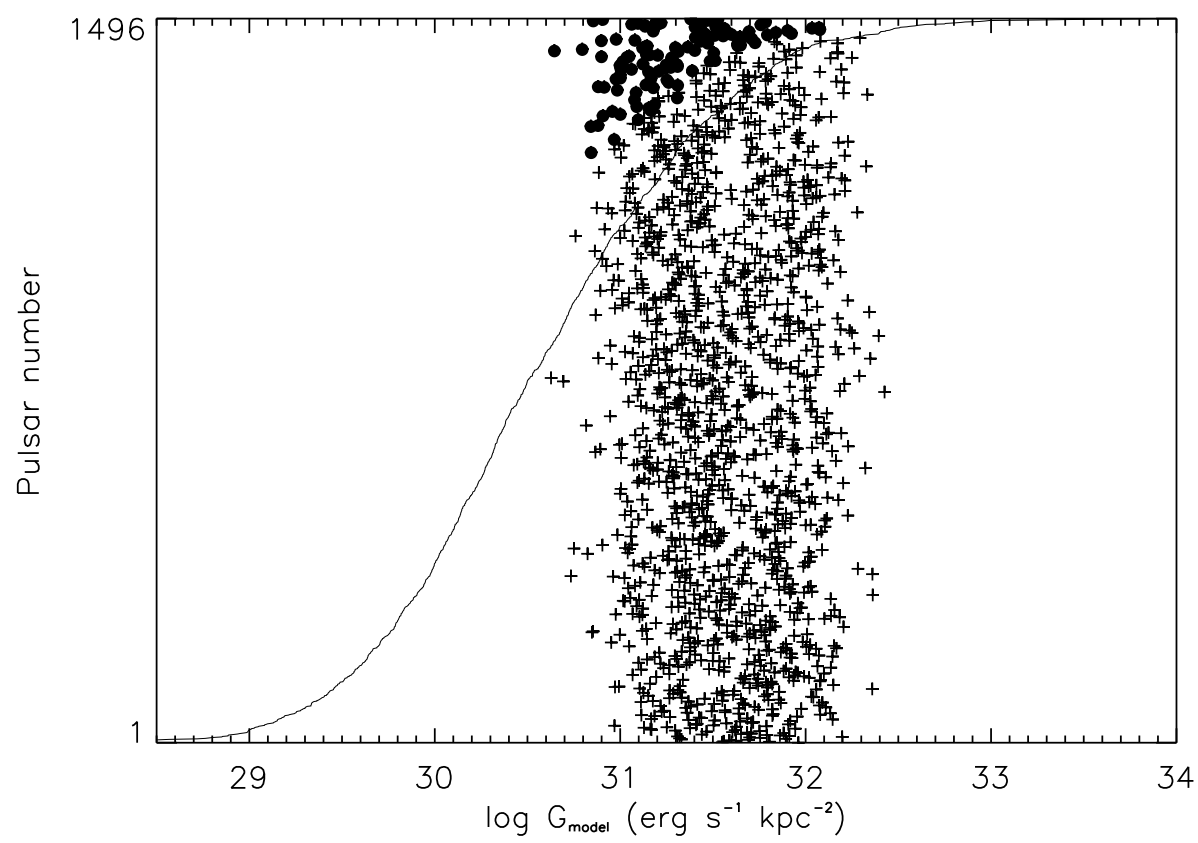

Figure 5.6: Model-estimated energy fluxes of 1496 non-recycled radio pulsars for the case of $f_{\Omega}=1$. The model-estimated fluxes are sorted according to decreasing order and connected by a thick line. Plus signs are the measured upper limit fluxes. The beaming analysis estimated 114 severe non-detections, which are located in the upper part of the figure and marked with filled circles. We define a severe non-detection as a measured upper limit that is $2 \sigma$ below the model prediction.

$100 \%$, where 1496 is the total number of pulsars in the sample) of the radio-detected pulsars have their radio beams within the gamma-ray beams and are potentially detectable in gamma rays. Figure 5.6 shows the measured upper limit fluxes of the known radio pulsars and their expected fluxes based on our best-fit luminosity law. Note that for the beaming analysis of this model, we use $\rho_{\gamma}$, which is independent of $\alpha$, as the model parameter. However, we use the $\alpha$ dependence on $\rho_{\gamma}$ in TPC and OG models below. By performing the above analysis, we constrained $\rho_{\gamma}=68(2)^{\circ}$. Assuming a uniform beam, the corresponding $\rho_{\gamma}$ implies that the average gamma-ray beaming solid angle is about $3.7(1) \pi$.

For outer magnetosphere models, we use the best-fit luminosity laws deter- 
mined for TPC and OG models. The luminosity law of the TPC model predicts 162 severe non-detections. In other words, $11 \%$ of the pulsars in the sample have their radio beams outside the gamma-ray beam. Note that for pulsars with estimated $\alpha$, we use that value directly to calculate the beaming solid angle of that pulsar. Fitting for the other pulsars, we find a best-fit $\alpha=77(4)^{\circ}$, implying $\rho_{\gamma, \mathrm{TPC}}=64^{\circ}$. The average beaming solid angle is then computed to be $\Omega_{\gamma}=3.6 \pi$, assuming a uniform beam. Note that the errors for $\rho_{\gamma}$ and $\Omega_{\gamma}$ are not quoted, because the derived $\rho_{\gamma}(\alpha)$ expressions for TPC and OG models are approximates and associated with large errors. The best-fit luminosity law for the OG model predicts 117 severe nondetections (i.e. about $92 \%$ of the upper-limit pulsars have their radio beams within the fan-like gamma-ray beams). The beaming analysis results in $\alpha=80(3)^{\circ}$, so that $\rho_{\gamma, \mathrm{OG}}=68^{\circ}$. This gives an average total solid angle swept out by the gamma-ray beam of $3.7 \pi$.

Note that Wang \& Hirotani (2011) predict an OG model death line for gammaray emission of $\log \dot{P}=-13.54+3.67 \log P$. Using only the 561 upper limits for pulsars satisfying this criterion, we find 69 severe non-detections, implying that $88 \%$ of the radio-detected pulsars have radio beams within the gamma-ray emission beams. The total solid angle swept out by the gamma-ray beam is $3.5 \pi$ with a uniform beam. 


\subsection{Properties and population analysis of GRPs with diffuse flux measurements}

As in MC00, we construct a model pulsar population in the Galaxy and then assume that some fraction of the Galactic diffuse flux is due to unresolved pulsars. We use our model-estimated flux in a given direction in the Galaxy and the measured diffuse flux in the same direction to place constraints on braking index $n$, magnetic field $B_{12}$, and initial spin period $P_{0}$ of the Galactic pulsar population.

First we need a Galactic pulsar spatial distribution. MC00 used a simple model with a Gaussian disk, exponential halo, and molecular ring. In contrast, we use a more accurate spatial distribution derived in Lorimer et al. (2006b) based on a sample of 1008 non-recycled pulsars from the Parkes multibeam survey. Then the pulsar number density $\rho(r, z)$ can be given as a function of radial distance from the Galactic center $r$ and the height from the Galactic plane $z$,

$$
\rho(r, z)=\rho_{0}\left(\frac{r}{d_{\odot}}\right)^{B} \exp \left(\frac{-|z|}{E}\right) \exp \left[-C\left(\frac{r-d_{\odot}}{d_{\odot}}\right)\right]
$$

where $E, B$, and $C$ are $0.18 \mathrm{kpc}, 1.9$, and 5.0 , respectively, and $\rho_{0}$ is a normalization constant. The distance to the Sun is taken to be $d_{\odot}=8.5 \mathrm{kpc}$. According to Ankay et al. (2004) and Lorimer et al. (1993), the Galactic pulsar birth rate is likely between $1 / 125 \mathrm{yr}^{-1}$ and $1 / 250 \mathrm{yr}^{-1}$. We assume a conservative pulsar birth rate of $1 / 100 \mathrm{yr}^{-1}$, implying the total number of pulsars in the Galaxy is $N_{\mathrm{psr}}=10^{8}$. Thus, we find the normalization constant by integrating 


$$
N_{\mathrm{psr}}=\int_{0}^{r_{0}} d r \int_{0}^{2 \pi} d \phi \int_{-z_{0}}^{z_{0}} d z r \rho(r, z)
$$

where $r_{0}=30 \mathrm{kpc}$ and $z_{0}=10 \mathrm{kpc}$, assuming that the Galaxy extends up to $30 \mathrm{kpc}$ radially and $10 \mathrm{kpc}$ above and below the plane. This integration returns $\rho_{0}=$ $4.05 \times 10^{5} \mathrm{kpc}^{-3}$. Note that this particular distribution is cylindrically symmetric; it does not depend on the azimuthal angle $\phi$, resulting in the same number of pulsars at a given radial distance for any $\phi$. Therefore, in order to compute the number of pulsars in a given Galactic direction, we convert the Galactic longitude $(l)$ and latitude $(b)$ to the above $(r, z)$ coordinates using

$$
\begin{aligned}
& x=D_{\mathrm{i}, \odot} \cos (b) \sin (l) \\
& y=d_{\odot}-D_{\mathrm{i}, \odot} \cos (b) \cos (l) \\
& z=D_{\mathrm{i}, \odot} \sin (b) \\
& r=\sqrt{x^{2}+y^{2}}
\end{aligned}
$$

where $D_{\mathrm{i}, \odot}$ is the distance with respect to the Sun. We can then calculate the flux from pulsars in a volume element $d v$ at a distance $D_{\mathrm{i}, \odot}$ for a given Galactic direction as $d G=d v \rho(r, z)\left\langle L_{\gamma} \mid B_{12}\right\rangle / 4 \pi f_{\Omega} D_{\mathrm{i}, \odot}^{2}$, where $\left\langle L_{\gamma} \mid B_{12}\right\rangle$ is the average luminosity of a pulsar for a given magnetic field. The expression for $\left\langle L_{\gamma} \mid B_{12}\right\rangle$ is given in Equation (C.1) and (C.2). To simplify the analysis, according to our reference model discussed in Section 5.5.1, we fixed $f_{\Omega}$ to be unity for all pulsars in the Galaxy. The number 
of pulsars within a unit volume can be written as $\rho(r, z) d v=A\left(D_{\mathrm{i}, \odot}\right) \rho(r, z) d D_{\mathrm{i}, \odot}$, where $A\left(D_{\mathrm{i}, \odot}\right)=\Omega_{\mathrm{b}} D_{\mathrm{i}, \odot}^{2}$, assuming a solid angle $\Omega_{\mathrm{b}}$ for the telescope beam centered on Galactic longitude $l$ and latitude $b$ with a uniform flux distribution across the beam. Then the model-estimated flux from pulsars along a Galactic direction $(l, b)$ can be calculated as

$$
\begin{aligned}
G\left(l, b, \Omega_{\mathrm{b}}\right)_{\text {model }} & =\int_{0}^{D_{\max }} d D_{\mathrm{i}, \odot} \frac{\left\langle L_{\gamma} \mid B_{12}\right\rangle}{4 \pi D_{\mathrm{i}, \odot}^{2}} A\left(D_{\mathrm{i}, \odot}\right) \rho(r, z) \\
& =\left\langle L_{\gamma} \mid B_{12}\right\rangle \frac{\Omega_{\mathrm{b}}}{4 \pi} \int_{0}^{D_{\max }} d D_{\mathrm{i}, \odot} \rho(r, z)
\end{aligned}
$$

where $D_{\max }$ is the distance of the furthest pulsar in a given Galactic direction $(l, b)$ and the telescope beam solid angle $\Omega_{\mathrm{b}}=2 \pi(1-\cos \theta)$, where $\theta$ is the angular radius of the beam. We assumed a $\theta$ of $1^{\circ}$ in order to ensure an isotropic flux distribution across the beam. Therefore, with equations (C.1), (5.7), (5.9), and (5.10), we can estimate the contribution from pulsars to the diffuse flux in a given Galactic direction.

We fit our model-estimated flux to the measured diffuse fluxes with a similar likelihood analysis as described in Section 5.3. In equation (C.1), we use our bestfit $a, b$, and $c$ from the reference model to estimate the average luminosity of a pulsar for a given model $\Theta$. We evaluate the average luminosity according to the log-normal and flat distributions of the magnetic field and the initial spin period of the population, respectively. In order to do this, we follow 


$$
<L_{\gamma} \mid B_{12}>=\frac{\int d P_{0, \mathrm{i}} \int d B_{\mathrm{i}}<L_{\gamma} \mid B_{\mathrm{i}}>\exp \left(-\left(\log B_{\mathrm{i}}-B_{0}\right)^{2} / 2 \sigma_{\mathrm{i}}^{2}\right)}{\int d P_{0, \mathrm{i}} \int d B_{\mathrm{i}} \exp \left(-\left(\log B_{\mathrm{i}}-B_{0}\right)^{2} / 2 \sigma_{\mathrm{i}}^{2}\right)}
$$

where $B_{\mathrm{i}} \in\left[10^{9}, 10^{15}\right] \mathrm{G}$ and $B_{0}=\log \left(B_{12} \times 10^{12}\right)$. We assumed $\sigma_{\mathrm{i}}=0.466$ by fitting a log-normal distribution to timing-derived surface magnetic fields of known nonrecycled pulsars. We kept this value fixed because our analysis is not sensitive to $\sigma_{\mathrm{i}}$. However, we fit for the peak of the log-normal distribution. Since the distribution of initial spin period $P_{0}$ of pulsars is not clearly understood (see Section 1.2.4), we assume that $P_{0}$ is bounded by $P_{0, \min }$ and $P_{0, \max }$, to be constrained in the analysis. In order to constrain $n, B_{12}, P_{0, \min }$ and $P_{0, \max }$, we follow a grid search for all four parameters while fitting the model-estimated diffuse flux to the measured diffuse flux with a step-like one-sided Gaussian function.

The contribution from pulsars to the Galactic diffuse flux is not well understood. Recent studies for high-latitude diffuse emission showed that the MSP population contributes a small fraction $(\sim 1 \%)$ of the Galactic diffuse emission (Ackermann et al., 2012; Grégoire \& Knödlseder, 2013). However, a similar study for Galactic plane emission has not been done. Therefore, to be conservative, we assume that a maximum of $10 \%$ of the diffuse flux is due to pulsars. Furthermore, we discuss how this fraction affects our constrained values. For a given model $\Theta$, i.e. $\left(n, B_{12}, P_{0, \min }, P_{0, \max }\right)$, the individual likelihood for the beam direction $(l, b)$ is given as 


$$
\mathcal{L}_{\mathrm{dif}, \mathrm{i}}(\Theta)=h_{\mathrm{i}}\left(G_{\mathrm{i}}, \Theta\right)
$$

where $h_{\mathrm{i}}\left(G_{\mathrm{i}}, \Theta\right)=\left(2 \pi \sigma_{\mathrm{i}}^{2}\right)^{-1 / 2} \exp \left(-\left(G\left(l, b, \Omega_{\mathrm{b}}, \Theta\right)_{\bmod , \mathrm{i}}-\epsilon_{\mathrm{d}} G(l, b)_{\operatorname{dif}, \mathrm{i}}\right) / 2 \sigma_{\mathrm{i}}^{2}\right)$ when $G\left(l, b, \Omega_{\mathrm{b}}, \Theta\right)_{\bmod }>\epsilon_{\mathrm{d}} G(l, b)_{\mathrm{dif}, \mathrm{i}}$. If $G\left(l, b, \Omega_{\mathrm{b}}, \Theta\right)_{\bmod }<\epsilon_{\mathrm{d}} G(l, b)_{\mathrm{dif}, \mathrm{i}}$ then the distribution $h_{\mathrm{i}}\left(G_{\mathrm{i}}, \Theta\right)=\left(2 \pi \sigma_{\mathrm{i}}^{2}\right)^{-1 / 2}$. Here, $G(l, b)_{\text {dif,i }}$ and $\sigma_{\mathrm{i}}$ are the measured diffuse flux and its error, respectively. We define $\epsilon_{\mathrm{d}}$ as the fraction of the diffuse flux that is due to pulsars. Then we determine the total likelihood of the model $\Theta$ from $\mathcal{L}_{\text {dif }}(\Theta)=\prod_{\mathrm{i}=1}^{N_{\text {beam }}} \mathcal{L}_{\text {dif,i }}(\Theta)$, where $N_{\text {beam }}$ is the number of diffuse flux measurements, and then follow the same analysis to calculate the PDFs of $n, B_{12}, P_{0, \min }$, and $P_{0, \max }$.

With the assumptions that $f_{\Omega}=1$ and $\epsilon_{\mathrm{d}}=0.1$, and assuming the bestfit parameters of $a, b$, and $c$ as determined in Section 5.5.1, we search the entire parameter space of $n, B_{12}, P_{0, \min }$ and $P_{0, \max }$ and calculate their PDFs, shown in Figure 5.7. We place a 95\%-confidence upper-limit on $n$ and $P_{0, \min }$ of 3.8 and $120 \mathrm{~ms}$, respectively, and a $95 \%$-confidence lower-limit on $B$ and $P_{0, \max }$ of $3.2 \times 10^{10} \mathrm{G}$ and $46 \mathrm{~ms}$, respectively. Changing $\epsilon_{\mathrm{d}}$ alters these limits slightly. For $\epsilon_{\mathrm{d}}=0.01$, we find a $95 \%$-confidence upper-limit on $n$ of 3.7 and a $95 \%$-confidence lower-limit on $B$ to be $2.2 \times 10^{10} \mathrm{G}$. Clearly, these limits are not constraining the pulsar population. In Figure 5.8, we show how the fraction of diffuse flux due to unidentified pulsars varies with $n$ and $B$. Note that the curves of $n$ and $B$ are determined according to the upper and lower limits at $95 \%$ confidence levels, respectively. We fixed $P_{0}$ at $100 \mathrm{~ms}$ to be consistent with the results of Popov \& Turolla (2012). We vary only one parameter at a time while keeping the other parameters at their typical 

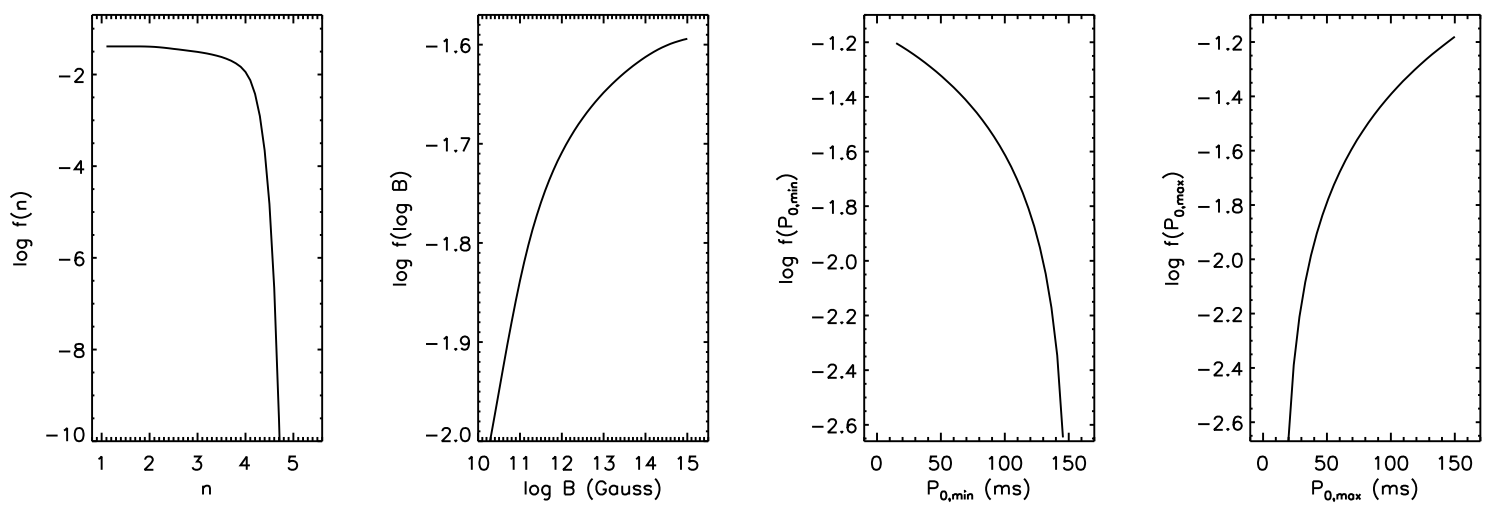

Figure 5.7: Marginalized PDFs for braking index $n$, surface magnetic field $B$, minimum limit of initial spin period $P_{0, \mathrm{~min}}$, and maximum limit of initial spin period $P_{0, \text { max }}$.

values of $n=2.5$ and $B=10^{12} \mathrm{G}$. Thus, it is clearly shown that the diffuse fraction due to pulsars is not constraining for typical parameters $n=2.5, B=10^{12} \mathrm{G}$, and $P_{0}=100 \mathrm{~ms}$.

Using Equation (C.4), we can estimate the flux distribution of GRPs in the Galaxy with the best-fit luminosity law and $n=2.5, B=10^{12} \mathrm{G}$, and $P_{0}=100 \mathrm{~ms}$ (see Figure 5.9). We derive two different model population predicted fluxes for $\epsilon_{\gamma}=0.75$ and 1 . We then predict the number of detectable pulsars using the LAT according to its sensitivity. These are discussed in the next section.

Furthermore, we model the luminosity distribution of GRPs in the Galaxy. Assuming that all the pulsars have $f_{\Omega}=1$, we use the corresponding best-fit parameters $a=1.43, b=0.40$, and $\log (c)=32.83$ (see Table 5.2), with $n=2.5$, $B=10^{12} \mathrm{G}$, and $P_{0}=100 \mathrm{~ms}$. Then we write the differential number of pulsars versus luminosity $d N_{\mathrm{psr}} / d L_{\gamma}=N_{\mathrm{psr}} f_{\mathrm{L}_{\gamma}}\left(L_{\gamma} \mid B_{12}\right)$, where $f_{\mathrm{L}_{\gamma}}\left(L_{\gamma} \mid B_{12}\right)$ is given in Equation (C.6). With a similar form of expression as given in Equation (C.5), but for the luminosity, we estimate the number of pulsars for a given luminosity. Figure 5.10 

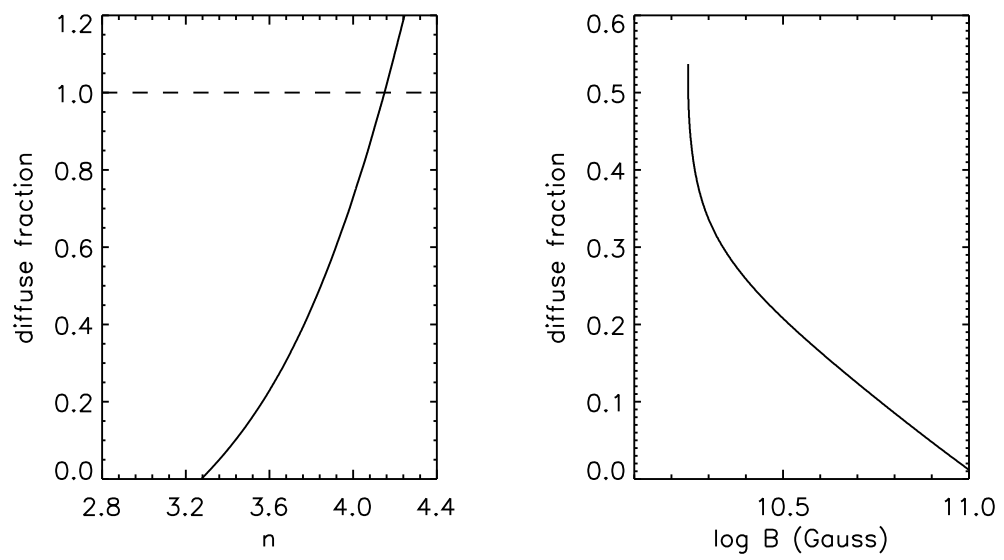

Figure 5.8: The fraction of Galactic diffuse flux due to unidentified pulsars as a function of braking index $n$ and surface magnetic field $B$. The curves of $n$ and $B$ are determined according to the upper and lower limits at $95 \%$ confidence levels. In each plot, one parameter is varied while keeping the other parameter fixed, at a values of 2.5 for $n$ or $10^{12} \mathrm{G}$ for $B$. The initial spin period $P_{0}$ is fixed at $100 \mathrm{~ms}$ throughout the fit. The dashed line shows where the fraction of diffuse flux due to pulsars reaches unity, showing that the range where $n>4.1$ is not allowed.

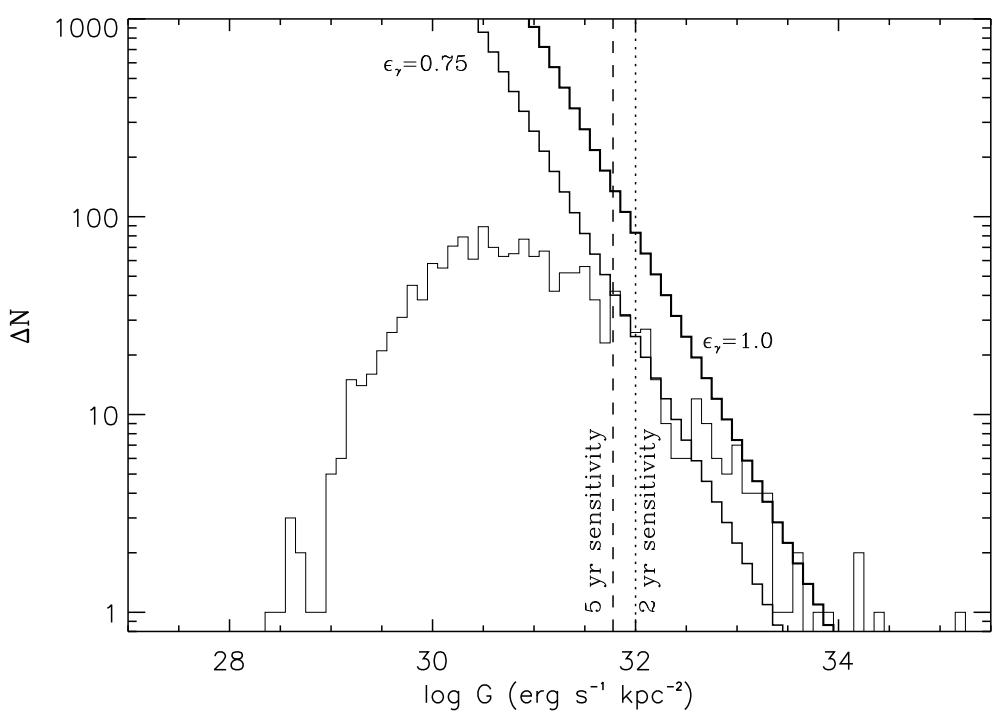

Figure 5.9: Predicted Fermi LAT energy fluxes of the GRP population model. The thin solid line is the model-predicted fluxes for identified non-recycled pulsars according to their period and period derivative with our best-fit luminosity law for $f_{\Omega}=1$. The two thick solid curves are the predicted fluxes for the model population of GRPs for two different maximum efficiencies $\epsilon_{\gamma}$. The dotted line is the LAT 2 year sensitivity for a point source in the Galactic-plane (Nolan et al., 2012). The dashed line is the expected LAT 5 year point source sensitivity. 


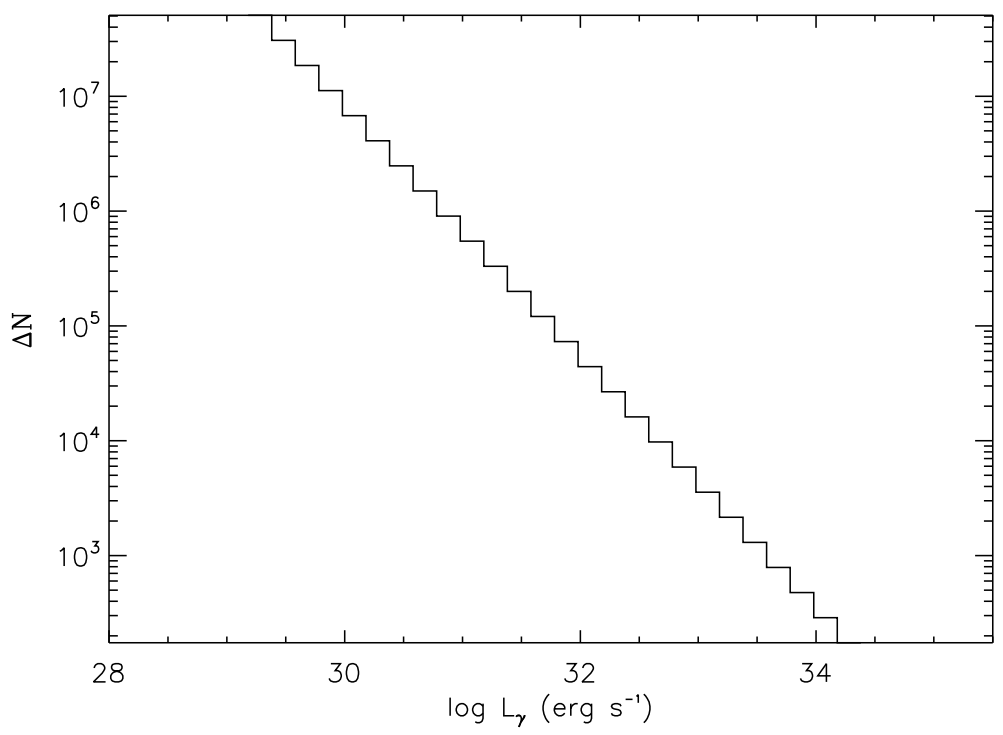

Figure 5.10: The luminosity distribution of GRPs in the Galaxy. The best-fit luminosity function is constrained to be $\log N\left(L_{\gamma}\right)=-1.09 \log L_{\gamma}+39.71$. Note that we assumed all the pulsars have a beaming factor of $f_{\Omega}=1$.

shows the Galactic luminosity distribution. The luminosity function is then fit with a power law as

$$
N\left(L_{\gamma}\right)=\left\{\begin{array}{cl}
k L_{\gamma}^{-m} & \text { for } L_{\gamma, \min }<L_{\gamma}<L_{\gamma, \max } \\
0 & \text { for otherwise }
\end{array}\right.
$$

where $m=1.09$ and $\log k=39.71$. The minimum and maximum luminosities $L_{\gamma, \min }=1.9 \times 10^{29} \mathrm{erg} \mathrm{s}^{-1}$ and $L_{\gamma, \max }=4.6 \times 10^{34} \mathrm{erg} \mathrm{s}^{-1}$ are determined from the oldest and the youngest pulsar in the Galaxy based on their spin periods, respectively.

\subsection{Discussion}

The best-fit luminosity laws show a significant improvement in uncertainties of the parameters compared to the results of previous works, MC00 and MC03, due 
to the larger number of GRPs in our sample. We find similar results assuming either $f_{\Omega}=1$ or a TPC or OG emission geometry (see Table 5.2). For all three of these models, the best-fit law is similar but not identical to $L_{\gamma} \propto \sqrt{\dot{E}}$. The OG model $\left(L_{\gamma} \propto P^{-a} \dot{P}_{15}^{b}\right.$ where $a=1.36 \pm 0.03$ and $\left.b=0.44 \pm 0.02\right)$ provides the best-fit to the data over all other models. This is consistent with the result of Watters \& Romani (2011) that the OG model is strongly preferred in order to explain the observed spin and pulse properties of GRPs. The data are not well fit through a PC model (see Table 5.2). We use the best-fit luminosity law for $f_{\Omega}=1$ model as the reference model for the upper-limit analysis and population model. The large chi-squared values associated with our fits, however, imply that the analyses are dominated by distance and beaming uncertainties. Some of the outliers in Figures 5.2, 5.4, and 5.5 may be associated with poor distance estimates. Parallax measurements for additional pulsars would dramatically improve our analysis.

Using flux upper limits, we determined the number of radio-detected pulsars that cannot be observed in gamma rays due to their large beam misalignment. The OG model predicts 117 such severe non-detections, or $8 \%$ of the population. Although our simple beam model does not perfectly represent gamma-ray beam geometries, it constrains the relationship of radio and gamma-ray beams and offers insights into the numbers of radio-loud and radio-quiet gamma-ray pulsars. Furthermore, the beam model predicts large beaming solid angles, implying that the pulsar gamma-ray emission covers almost the entire sky, consistent with realistic outer magnetosphere emission models.

Using LAT diffuse fluxes and our population model, we can constrain some 
properties of the GRP population such as $n, B_{12}$, and $P_{0}$. A $2 \sigma$ upper limit on the average $n$ and a $2 \sigma$ lower limit on the average $B_{12}$ of the population are 3.8 and $3.2 \times 10^{10} \mathrm{G}$, respectively. While these are not yet physically constraining, they should become so with time as more point sources are discovered and more accurate diffuse background models are developed.

According to our reference luminosity law along with the periods and period derivatives of known pulsars, the mean of the gamma-ray flux distribution is about $2 \times 10^{32} \mathrm{erg} \mathrm{s}^{-1} \mathrm{kpc}^{-2}$ (see Figure 5.9). We then calculate the number of LAT-detectable pulsars based on our Galactic model with a maximum gamma-ray efficiency of $\epsilon_{\gamma}=1$. Given the LAT sensitivity for a point source in the Galactic plane $\left(|l|<1^{\circ}\right)$ of $1 \times 10^{32} \mathrm{erg} \mathrm{s}^{-1} \mathrm{kpc}^{-2}$ from the $2 \mathrm{FGL}$ based on the first 24 months of data (Nolan et al., 2012), it is capable of detecting about 380 non-recycled pulsars as point sources, including 150 currently identified pulsars. According to the 2FGL, pulsations from 83 pulsars have been reported ${ }^{6}$. With the expected 5-year point source sensitivity of $6 \times 10^{31} \mathrm{erg} \mathrm{s}^{-1} \mathrm{kpc}^{-2}$, scaled on the 2-year sensitivity (i.e., $1 \times 10^{32} \mathrm{erg} \mathrm{s}^{-1} \mathrm{kpc}^{-2}$ times $\left.\sqrt{2 / 5}\right)$, it is capable of detecting emission from about 620 pulsars, including about 220 currently identified pulsars. However, the model assumptions significantly impact the predictions. For instance, a smaller beaming fraction would imply smaller numbers of detectable GRPs.

\footnotetext{
${ }^{6}$ About 77 non-recycled GRPs have been detected with the LAT https://confluence.slac.stanford.edu/display/GLAMCOG/Public+List+of+LATDetected+Gamma-Ray+Pulsars
} 


\section{Chapter 6}

\section{Conclusions and Future work}

\subsection{PSR J0737-3039A/B and future work related with the system}

In Chapter 2, we analyzed the GBT data of PSR J0737-3039B since 2003 December. We found that the radio emission of the pulsar evolved dramatically over time. The pulse profile evolved from a single to a double peak and the mean flux density decreased significantly within five years of observation, culminating in the radio emission disappearance towards our line of sight since 2008 March. Based on geodetic spin precession, we explained pulse profile variation qualitatively and then determined pulsar B's geometry. With the best-fit parameters, the model predicts the reappearance from the radio emission of $\mathrm{B}$ is expected to happen anytime between 2014 and 2035. However, this simple model was unable to explain the observed flux decrease with time.

In Chapter 3, we developed a wind-magnetosphere interaction model and determined the boundary of pulsar B. We found that the size of the magnetosphere is much smaller than the light cylinder radius $\left(R_{L C}\right)$, less than $0.3 R_{L C}$. However, it is inconsistent with the size determined from the eclipses of $\mathrm{A}\left(\sim 0.1 R_{L C}\right)$. This discrepancy can be explained if there is lower particle density in the outer regions of the magnetosphere compared to the inner regions, so that the radiation of A can penetrate only the outer regions. We modeled the radio emission from this distorted 
magnetosphere with the well-derived geometry and estimated the emission altitudes of the pulsar. We found that the radio emission altitude varies across the orbit as well as with time. These results are consistent with the emission altitudes of non-recycled pulsars.

In Chapter 4, we determined the geometry of pulsar A with a simple circular double-pole radio emission model based on geodetic spin precession. Then we developed a vacuum retarded dipole magnetosphere with a polar cap configuration to model the radio beam. We estimated radio emission altitudes and beam size by fitting the synthesized pulse profiles to observed profiles. Then, combining the estimated geometries of both pulsars A and B, we estimated the variation of the orientation of the spin axes of the two pulsars over time. For the first time this relative angle was constrained in a DNS system. This is very important information in studying binary formation/evolution involving supernova natal kicks.

Based on these useful results from the Double Pulsar, we can extensively study radio emission and geometries of pulsars in the future. This will lead us to probe the real magnetospheric structure of NSs. As discussed in early chapters, we generally use static or retarded dipole fields and assume that the magnetospheres are in a vacuum or filled with particles in force-free conditions. However, we have not encountered a situation that enables us to study the behavior of the plasma within the magnetosphere. More than eight years of Double Pulsar data can be used to analyze the eclipses of A greater in detail with the estimated geometry and the nonuniform particle density of B. Including magnetic interaction, this model will be fit for observed pulse profiles and emission regions of both pulsars. Current and future 
high resolution GBT observations of A (and B, when it returns) are important because we need to sample the entire magnetosphere (either directly or through the eclipses) to understand the emission pattern of the pulsar. This empirical model will explain the characteristics of emission of any radio pulsar in general. This model will definitely be useful if we discover more DNSs or NS-black hole binary systems in the future.

Furthermore, the Double Pulsar provides an unique laboratory for pulsar timing. Currently, we time B by calculating TOAs during its weak phases, because the pulse profile is more stable. In the future, we can calculate TOAs by using the bright phases with templates generated using our beaming model. This may improve the timing solution, resulting in more precisely calculated individual masses of the two NSs than ever. This may allow us to measure the $2^{\text {nd }}$-order Post-Newtonian correction to the rate of periastron advance. This allows us to determine the moment of inertia of pulsar A. This would be the first measured NS moment of inertia and would help in understanding the NS equation-of-state. This is one of the primary unanswered questions in pulsar astrophysics.

\subsection{Fermi gamma-ray analysis and future work}

In Chapter 5, we explored Fermi gamma-ray detections, upper limits, and diffuse measurements to analyze Galactic gamma-ray pulsar population. We constrained the luminosity law for gamma-ray pulsars based on the LAT detections. The OG model provides the best-fit luminosity for gamma-ray pulsars. This closely 
follows the form of $L_{\gamma} \propto \sqrt{\dot{E}}$. This best-fit result along with upper limits of radio-detected pulsars showed that about $92 \%$ of the radio-detected pulsars have gamma-ray beams that intersect our line of sight. By modeling the misalignment of radio and gamma-ray beams of these pulsars, we determined an average gammaray beaming solid angle of about $3.7 \pi$ for the OG model. Using LAT diffuse flux measurements, we placed limits on some properties of Galactic gamma-ray pulsar population such as braking index, and surface magnetic field. Finally, we predicted the number of non-recycled pulsars detectable by the LAT based on our population model. With the expected five-year sensitivity, about 620 non-recycled pulsars are detectable from the LAT, including about 220 currently identified radio pulsars. We note that these predictions significantly depend on our model assumptions.

As described in the luminosity analysis, an accurate distance to the pulsar is important. We used available parallax measurements, HI features and NE2001 model to estimate the distance. In the future, with the more reliable new electron density model NE2012, we can update the current gamma-ray luminosity law. Furthermore, we can use an improved Galactic spatial distribution including distributions of pulsar spins, age, and magnetic inclinations to construct an advanced population model. This will make reliable predictions for the Fermi mission and be useful for next generation high-energy missions. Since MSPs are important targets for the Fermi mission, we can perform a similar analysis for MSPs to understand their luminosity and emission properties. Then we will be able to place useful constrains on Galactic diffuse emission from both CPs and MSPs in the future. 


\section{Bibliography}

Abbott, B., et al. 2008, Phys. Rev. D, 78, 042002

Abdo, A. A., et al. 2009, ApJ, 706, 1331

—. 2010a, ApJS, 188, 405

—. 2010b, ApJ, 720, 26

—. 2010c, ApJ, 712, 1209

-. 2010d, ApJS, 187, 460

Ackermann, M., et al. 2012, Phys. Rev. D, 85, 083007

Anderson, M., Hirschmann, E. W., Lehner, L., Liebling, S. L., Motl, P. M., Neilsen, D., Palenzuela, C., \& Tohline, J. E. 2008, Phys. Rev. Lett., 100, 191101

Ankay, A., Guseinov, O. H., \& Tagieva, S. O. 2004, Astronomical and Astrophysical Transactions, 23, 503

Arzoumanian, Z. 1996, Thesis, Princeton Univ.

Arzoumanian, Z., Chernoff, D. F., \& Cordes, J. M. 2002, ApJ, 568, 289

Atwood, W. B., et al. 2009, ApJ, 697, 1071

Bai, X.-N., \& Spitkovsky, A. 2010a, ApJ, 715, 1282

—. 2010b, ApJ, 715, 1270

Barker, B. M., \& O'Connell, R. F. 1975, Phys. Rev. D, 12, 329

Blandford, R., \& Teukolsky, S. A. 1976, ApJ, 205, 580

Blaskiewicz, M., Cordes, J. M., \& Wasserman, I. 1991, ApJ, 370, 643

Boerner, G., Ehlers, J., \& Rudolph, E. 1975, A\&A, 44, 417

Breton, R. P., et al. 2008, Science, 321, 104

Burgay, M., et al. 2003, Nature, 426, 531

-. 2005, ApJ, 624, L113

Camilo, F., et al. 2012, ApJ, 746, 39

Caswell, J. L., McClure-Griffiths, N. M., \& Cheung, M. C. M. 2004, MNRAS, 352, 1405

Chapman, R., Priddey, R. S., \& Tanvir, N. R. 2009, MNRAS, 395, 1515 
Cheng, A. F., \& Ruderman, M. A. 1977, ApJ, 216, 865

Cheng, K. S., Ho, C., \& Ruderman, M. 1986, ApJ, 300, 522

Chiang, J., \& Romani, R. W. 1992, ApJ, 400, 629

-. 1994, ApJ, 436, 754

Clifton, T., \& Weisberg, J. M. 2008, ApJ, 679, 687

Coles, W. A., McLaughlin, M. A., Rickett, B. J., Lyne, A. G., \& Bhat, N. D. R. 2005, ApJ, 623, 392

Contopoulos, I., \& Spitkovsky, A. 2006, ApJ, 643, 1139

Cordes, J. M., \& Lazio, T. J. W. 2002, astro-ph/0207156

Cordes, J. M., Rankin, J. M., \& Backer, D. C. 1978, ApJ, 223, 961

Damour, T., \& Deruelle, N. 1986, Ann. Inst. H. Poincaré (Physique Théorique), 44, 263

Damour, T., \& Taylor, J. H. 1992, Phys. Rev. D, 45, 1840

Daugherty, J. K., \& Harding, A. K. 1982, ApJ, 252, 337

Demorest, P. B., Pennucci, T., Ransom, S. M., Roberts, M. S. E., \& Hessels, J. W. T. 2010, Nature, 467, 1081

Desvignes, G., Kramer, M., Cognard, I., Kasian, L., van Leeuwen, J., Stairs, I., \& Theureau, G. 2013, in IAU Symposium, Vol. 291, IAU Symposium, 199-202

Deutsch, A. J. 1955, Annales d'Astrophysique, 18, 1

Dodson, R., Legge, D., Reynolds, J. E., \& McCulloch, P. M. 2003, ApJ, 596, 1137

Dyks, J., \& Harding, A. K. 2004, ApJ, 614, 869

Dyks, J., \& Rudak, B. 2003, ApJ, 598, 1201

Dyks, J., Rudak, B., \& Harding, A. K. 2004, ApJ, 607, 939

Espinoza, C. M., Lyne, A. G., Kramer, M., Manchester, R. N., \& Kaspi, V. M. 2011, ApJ, 741, L13

Faherty, J., Walter, F. M., \& Anderson, J. 2007, Ap\&SS, 308, 225

Farr, W. M., Kremer, K., Lyutikov, M., \& Kalogera, V. 2011, ApJ, 742, 81

Faucher-Giguère, C.-A., \& Kaspi, V. M. 2006, ApJ, 643, 332 
Ferdman, R. D., et al. 2008, in American Institute of Physics Conference Series, Vol. 983, 40 Years of Pulsars: Millisecond Pulsars, Magnetars and More, ed. C. Bassa, Z. Wang, A. Cumming, \& V. M. Kaspi, 474-478

Ferdman, R. D., et al. 2013, ApJ, 767, 85

Fierro, J. M., et al. 1995, ApJ, 447, 807

Freire, P. C. C., Wex, N., Kramer, M., Lorimer, D. R., McLaughlin, M. A., Stairs, I. H., Rosen, R., \& Lyne, A. G. 2009, MNRAS, 396, 1764

Gangadhara, R. T. 2004, ApJ, 609, 335

Gangadhara, R. T., \& Gupta, Y. 2001, ApJ, 555, 31

Gangadhara, R. T., \& Thomas, R. M. C. 2006, astro-ph/0604559

Gil, J., Gronkowski, P., \& Rudnicki, W. 1984, A\&A, 132, 312

Gil, J., Kijak, J., \& Zycki, P. 1993, A\&A, 272, 207

Gil, J., \& Kijak, K. 1993, A\&A, 273, 563

Gil, J. A., \& Snakowski, J. K. 1990a, A\&A, 234, 237

-. 1990b, A\&A, 234, 269

Goldreich, P., \& Julian, W. H. 1969, ApJ, 157, 869

Górski, K. M., Hivon, E., Banday, A. J., Wandelt, B. D., Hansen, F. K., Reinecke, M., \& Bartelmann, M. 2005, ApJ, 622, 759

Gould, D. M., \& Lyne, A. G. 1998, MNRAS, 301, 235

Gourgouliatos, K. N., Lyutikov, M., Lomiashvili, D., \& Perera, B. B. P. 2011, in American Institute of Physics Conference Series, Vol. 1357, American Institute of Physics Conference Series, 304-305

Grégoire, T., \& Knödlseder, J. 2013, ArXiv e-print, 1305.1584

Guillemot, L., et al. 2013, ApJ, 768, 169

Gupta, Y., \& Gangadhara, R. T. 2003, ApJ, 584, 418

Harding, A. K., DeCesar, M. E., Miller, M. C., Kalapotharakos, C., \& Contopoulos, I. 2011, ArXiv e-prints, 1111.0828

Harding, A. K., Stern, J. V., Dyks, J., \& Frackowiak, M. 2008, ApJ, 680, 1378

Hessels, J. W. T., Ransom, S. M., Stairs, I. H., Freire, P. C. C., Kaspi, V. M., \& Camilo, F. 2006, Science, 311, 1901 
Hewish, A., Bell, S. J., Pilkington, J. D. H., Scott, P. F., \& Collins, R. A. 1968, Nature, 217, 709

Hotan, A. W., Bailes, M., \& Ord, S. M. 2005, ApJ, 624, 906

Hulse, R. A., \& Taylor, J. H. 1975, ApJ, 195, L51

Ioka, K., \& Taniguchi, K. 2000, ApJ, 537, 327

Jackson, J. D. 1962, Classical Electrodynamics (Wiley)

Johnston, S., Hobbs, G., Vigeland, S., Kramer, M., Weisberg, J. M., \& Lyne, A. G. 2005, MNRAS, 364, 1397

Kalapotharakos, C., Harding, A. K., Kazanas, D., \& Contopoulos, I. 2012a, ApJ, $754, \mathrm{~L} 1$

Kalapotharakos, C., Kazanas, D., Harding, A., \& Contopoulos, I. 2012b, ApJ, 749, 2

Kaspi, V. M., Taylor, J. H., \& Ryba, M. 1994, ApJ, 428, 713

Kastaun, W., Galeazzi, F., Alic, D., Rezzolla, L., \& Font, J. A. 2013, ArXiv e-prints, 1301.7348

Keith, M. J., Kramer, M., Lyne, A. G., Eatough, R. P., Stairs, I. H., Possenti, A., Camilo, F., \& Manchester, R. N. 2009, MNRAS, 393, 623

Kerr, M. 2010, PhD thesis, University of Washington

Kijak, J., \& Gil, J. 1997, MNRAS, 288, 631

Kijak, J., \& Gil, J. 2003, ApJ, 397, 969

Kijal, J., \& Gil, J. 1998, MNRAS, 299, 855

Kramer, M. 1998, ApJ, 509, 856

—. 2010, ArXiv e-prints, 1008.5032

Kramer, M., Lyne, A. G., Hobbs, G., Löhmer, O., Carr, P., Jordan, C., \& Wolszczan, A. 2003, ApJ, 593, L31

Kramer, M., \& Stairs, I. H. 2008, ARA\&A, 46, 541

Kramer, M., Xilouris, K. M., Lorimer, D. R., Doroshenko, O., Jessner, A., Wielebinski, R., Wolszczan, A., \& Camilo, F. 1998, ApJ, 501, 270

Kramer, M., et al. 2006, Science, 314, 97

Landecker, T. L., Roger, R. S., \& Higgs, L. A. 1980, A\&AS, 39, 133 
Lattimer, J. M., \& Prakash, M. 2007, PhR, 442, 109

Lemoine-Goumard, M., et al. 2011, A\&A, 533, A102

Li, J., Spitkovsky, A., \& Tchekhovskoy, A. 2012, ApJ, 746, 60

Lorimer, D. R., Bailes, M., Dewey, R. J., \& Harrison, P. A. 1993, MNRAS, 263, 403

Lorimer, D. R., \& Kramer, M. 2005, Handbook of Pulsar Astronomy (Cambridge University Press)

Lorimer, D. R., et al. 2006a, ApJ, 640, 428

—. 2006b, MNRAS, 372, 777

Luo, Q., \& Melrose, D. B. 1992, MNRAS, 258, 616

Luo, Q., \& Melrose, D. B. 1995, MNRAS, 276, 372

Lyne, A. G., \& Manchester, R. N. 1988, MNRAS, 234, 477

Lyne, A. G., \& Smith, F. G. 2004, Pulsar Astronomy, 3rd ed. (Cambridge: Cambridge University Press)

Lyne, A. G., et al. 2004, Science, 303, 1153

Lyutikov, M. 2004, MNRAS, 353, 1095

—. 2005, MNRAS, 362, 1078

Lyutikov, M., \& Thompson, C. 2005, ApJ, 634, 1223

Magalhaes, N. S., Miranda, T. A., \& Frajuca, C. 2012, ApJ, 755, 54

Manchester, R. N., et al. 2005, ApJ, 621, L49

—. 2010, ApJ, 710, 1694

McLaughlin, M. A., \& Cordes, J. M. 2000, ApJ, 538, 818

—. 2003, astro-ph/0310748

McLaughlin, M. A., et al. 2004a, ApJ, 616, L131

-. 2004b, ApJ, 613, L57

Melrose, D. B. 1992, in The Magnetospheric Structure and Emission Mechanisms of Radio Pulsars, IAU Colloquium 128, ed. T. H. Hankins, J. M. Rankin, \& J. A. Gil (Zielona Góra, Poland: Pedagogical University Press), 306-315

Melrose, D. B. 1995, ApA, 16, 137 
Michel, F. C. 1991, Theory of Neutron Star Magnetospheres (Chicago: University of Chicago Press)

Migliazzo, J. M., Gaensler, B. M., Backer, D. C., Stappers, B. W., van der Swaluw, E., \& Strom, R. G. 2002, ApJ, 567, L141

Mitra, D., \& Rankin, J. M. 2011, ApJ, 727, 92

Moffett, D. A., \& Hankins, T. H. 1996, ApJ, 468, 779

Muslimov, A. G., \& Harding, A. K. 2003, ApJ, 588, 430

—. 2004, ApJ, 606, 1143

Ng, C.-Y., \& Romani, R. W. 2008, ApJ, 673, 411

Nolan, P. L., et al. 2012, ApJS, 199, 31

Noutsos, A., et al. 2011, ApJ, 728, 77

Palenzuela, C., Lehner, L., Ponce, M., Liebling, S. L., Anderson, M., Neilsen, D., \& Motl, P. 2013, ArXiv e-prints, 1301.7074

Parent, D., et al. 2011, ApJ, 743, 170

Perera, B. B. P., Lomiashvili, D., Gourgouliatos, K. N., McLaughlin, M. A., \& Lyutikov, M. 2012, ApJ, 750, 130

Perera, B. B. P., et al. 2010, ApJ, 721, 1193

Phinney, E. S. 1991, ApJ, 380, L17

Pierbattista, M., Grenier, I. A., Harding, A. K., \& Gonthier, P. L. 2012, A\&A, 545, A42

Pilkington, J. D. H., Hewish, A., Bell, S. J., \& Cole, T. W. 1968, Nature, 218, 126

Piro, A. L. 2012, ApJ, 755, 80

Pletsch, H. J., et al. 2012, ApJ, 744, 105

Popov, S. B., \& Turolla, R. 2012, Ap\&SS, 197

Radhakrishnan, V., \& Cooke, D. J. 1969, Astrophys. Lett, 3, 225

Rankin, J. M. 1983a, ApJ, 274, 333

-. 1983b, ApJ, 274, 359

-. 1990, ApJ, 352, 247

-. 1993a, ApJ, 405, 285 
—. 1993b, ApJS, 85, 145

Ray, P. S., et al. 2011, ApJS, 194, 17

Rezzolla, L., Giacomazzo, B., Baiotti, L., Granot, J., Kouveliotou, C., \& Aloy, M. A. 2011, ApJ, 732, L6

Roberts, M. S. E., Romani, R. W., \& Johnston, S. 2001, ApJ, 561, L187

Romani, R. W. 1996, ApJ, 470, 469

Romani, R. W., Kerr, M., Craig, H. A., Johnston, S., Cognard, I., \& Smith, D. A. 2011, ApJ, 738, 114

Ruderman, M. A., \& Sutherland, P. G. 1975, Astrophys. J., 196, 51

Spitkovsky, A. 2006, ApJ, 648, L51

Stairs, I. H. 2008, in American Institute of Physics Conference Series, Vol. 983, 40 Years of Pulsars: Millisecond Pulsars, Magnetars and More, ed. C. Bassa, Z. Wang, A. Cumming, \& V. M. Kaspi, 424-432

Stairs, I. H., Thorsett, S. E., \& Arzoumanian, Z. 2004, Phys. Rev. Lett., 93, 141101

Stairs, I. H., Thorsett, S. E., Taylor, J. H., \& Arzoumanian, Z. 2000, in Astronomical Society of the Pacific Conference Series, Vol. 202, IAU Colloq. 177: Pulsar Astronomy - 2000 and Beyond, ed. M. Kramer, N. Wex, \& R. Wielebinski, 121

Takata, J., Wang, Y., \& Cheng, K. S. 2011a, ApJ, 726, 44

—. 2011b, MNRAS, 415, 1827

Taylor, J. H., \& Weisberg, J. M. 1982, ApJ, 253, 908

—. 1989, ApJ, 345, 434

The Fermi-LAT collaboration. 2013, ArXiv e-prints, 1305.4385

Theureau, G., et al. 2011, A\&A, 525, A94

Thompson, D. J. 2004, in Astrophysics and Space Science Library, Vol. 304, Cosmic Gamma-Ray Sources, ed. K. S. Cheng \& G. E. Romero, 149

Thompson, D. J., et al. 1994, ApJ, 436, 229

Thorsett, S. E., Dewey, R. J., \& Stairs, I. H. 2005, ApJ, 619, 1036

Wang, R.-B., \& Hirotani, K. 2011, ApJ, 736, 127

Watters, K. P., \& Romani, R. W. 2011, ApJ, 727, 123 
Watters, K. P., Romani, R. W., Weltevrede, P., \& Johnston, S. 2009, ApJ, 695, 1289

Weisberg, J. M., Nice, D. J., \& Taylor, J. H. 2010, ApJ, 722, 1030

Weisberg, J. M., Romani, R. W., \& Taylor, J. H. 1989, ApJ, 347, 1030

Weisberg, J. M., \& Taylor, J. H. 2002, ApJ, 576, 942

Weisberg, J. M., \& Taylor, J. H. 2005, in Astronomical Society of the Pacific Conference Series, Vol. 328, Binary Radio Pulsars, ed. F. A. Rasio \& I. H. Stairs, 25

Weisskopf, M. C., et al. 2011, ApJ, 743, 74

Weltevrede, P., Johnston, S., \& Espinoza, C. M. 2011, MNRAS, 411, 1917

Weltevrede, P., \& Wright, G. 2009, MNRAS, 395, 2117

Weltevrede, P., et al. 2010, ApJ, 708, 1426

Wongwathanarat, A., Janka, H.-T., \& Müller, E. 2013, A\&A, 552, A126

Yadigaroglu, I.-A. G. 1997, PhD thesis, Stanford University 


\section{Appendix A}

\section{Derivation of the elliptical beam width corresponding to CW08}

In this beam model, we derive equations after projecting all the trajectories on the assumed unit sphere to the X-Y plane (see Figure 2.14 (a)). Since the halfopening angle of the beams is small, we use $2 \mathrm{D}$ trigonometry in the derivation.

Let $a$ and $b$ be the semi-major and semi-minor axes of the elliptical beam. We choose a coordinate system at the center of the projected beam in which $a$ is along the $x^{\prime}$ axis and $b$ is along the $y^{\prime}$ axis (see Figure A.1). Then we write the general expression for the ellipse as

$$
\frac{x^{\prime 2}}{a^{2}}+\frac{y^{\prime 2}}{b^{2}}=1 \Rightarrow y^{\prime 2}=b^{2}\left(1-\frac{x^{\prime 2}}{a^{2}}\right) \text {. }
$$

Then the projected angular radius of the elliptical beam with respect to the line-ofsight can be written as

$$
\rho^{2}(n)=x^{\prime 2}+y^{\prime 2}=x^{\prime 2}\left(1-\frac{b^{2}}{a^{2}}\right)+b^{2}
$$

With the assumption that the angular radius is very small, we can write $n(t) \approx$ $\sin \alpha+x^{\prime}$. This gives $x^{\prime}=n(t)-\sin \alpha$. Therefore, we can rewrite the expression for the angular radius as 


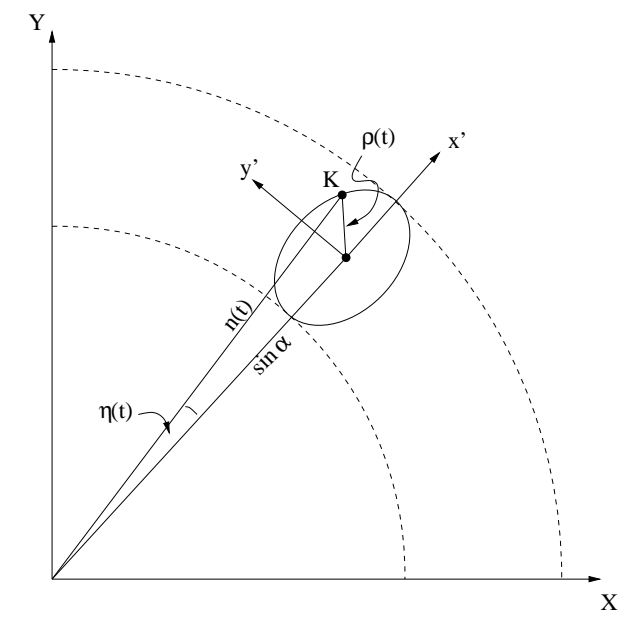

Figure A.1: After projecting all the trajectories on the assumed unit sphere onto the orbital plane. The point K shows the line-of-sight on the projected beam. Here $n(t)=\sqrt{n_{x}^{2}(t)+n_{y}^{2}(t)}$ and $\rho(t)$ is the projected angular radius of the beam.

$$
\rho(n)=\sqrt{(n(t)-\sin \alpha)^{2}\left(1-\frac{b^{2}}{a^{2}}\right)+b^{2}}
$$

where $n(t)$ is the projected line-of-sight onto the orbital plane, $n(t)=\sqrt{n_{x}^{2}(t)+n_{y}^{2}(t)}$. Now we can relate the two quantities $\rho(n)$ and $\eta(t)$ with the trigonometric relation $\rho^{2}(n)=n^{2}+\sin ^{2} \alpha-2 n \sin \alpha \cos \eta$. Then the expression for $\eta$ is obtained

$$
\eta(t)=\arccos \left(\frac{n^{2}(t)+\sin ^{2} \alpha-[n(t)-\sin \alpha]^{2}\left[1-R_{j}^{2}\right]+b_{j}^{2}}{2 n(t) \sin \alpha}\right)
$$

and this is equation (2.4) in the text. Then the projected pulse width can be written as

$$
w(t)=\arcsin \left(2 \sin \eta(t) \sqrt{\left[n_{x}(t)\right]^{2}+\left[n_{y}(t)\right]^{2}}\right)
$$

which is the equation (2.2). 


\section{Appendix B}

\section{Derivation of the new elliptical beam}

Here we derive an equation for the pulse profile width of an elliptical hollow cone beam in 3D, independent to the CW08 model. Assume an elliptical hollow cone beam where the vertex coincides with the center of the neutron star (see Figure B.1) and the angular radius of the cone is small enough to use 2D trigonometry. First consider the cross section of the beam, which is a two-dimensional ellipse where its center is located at the axis of the hollow cone. Then we can relate the pulse width $\eta^{\prime}$ (in units of length), semi-major $a$, and -minor $b$ axes with the general equation of an ellipse as

$$
\eta^{\prime}=R \sin \theta=\frac{a b \sin \theta}{\sqrt{(b \cos \theta)^{2}+(a \sin \theta)^{2}}} .
$$

Then the angle $\theta$ can be expressed in terms of $\eta^{\prime}$ and $r$

$$
\tan \theta=\frac{\eta^{\prime}}{r} .
$$

Then substitute $\theta$ to the previous equation and solve for the width

$$
\eta^{\prime}=\frac{1}{a} \sqrt{(a b)^{2}-(b r)^{2}} .
$$

Let $h$ be the height of the cone, $H$ the emission height and $\chi$ the ellipticity, 


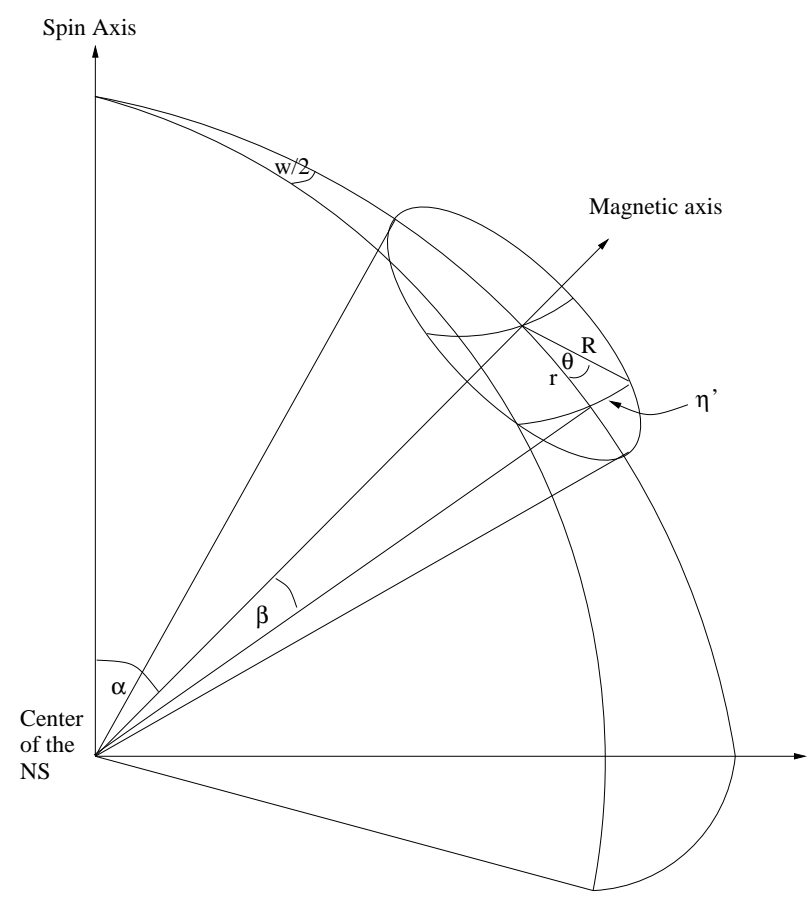

Figure B.1: Schematic view of the elliptical beam. The NS is at the origin of the coordinate system.

then we can write

$$
\begin{aligned}
& r=h \tan \beta \\
& h=H \cos \rho_{a, j} \\
& \chi=\frac{a}{b}
\end{aligned}
$$

where $\beta$ is the closest approach of the line-of-sight (i.e. impact parameter) and $\rho_{a, j}$ is the angular radius of the beam across the semi-major axis. Then $\eta^{\prime}$ is reduced to

$$
\eta^{\prime}=\frac{H}{\chi} \sqrt{\sin ^{2} \rho_{a, j}-\cos ^{2} \rho_{a, j} \tan ^{2} \beta} .
$$


Now we can write $\eta^{\prime}$ is in units of degrees $\left(\rho_{l, j} H=\eta^{\prime}\right)$ and express

$$
\rho_{l, j}=\frac{1}{\chi} \sqrt{\sin ^{2} \rho_{a, j}-\cos ^{2} \rho_{a, j} \tan ^{2} \beta} .
$$

This is Equation (2.7) of the text. In order to relate $\rho_{l, j}$ with pulse profile width $(w)$, we can use spherical geometry and express

$$
w=2 \arccos \left(\frac{\cos \left(\rho_{l, j}\right)-\cos ^{2}(\alpha+\beta)}{\sin ^{2}(\alpha+\beta)}\right)
$$

where $\alpha$ is the magnetic inclination with respect to the spin axis. Here, $w$ is in units of radians and this is Equation (2.7). 


\section{Appendix C}

\section{Gamma-ray pulsar population}

For a given luminosity law with an assumed spin-down law $\dot{\Omega} \propto \Omega^{n}$, where $\Omega$ is the spin frequency and $n$ is the braking index, the average luminosity of a pulsar for a given magnetic field strength can be written as

$$
\left\langle L_{\gamma} \mid B_{12}\right\rangle=\frac{2 c B_{12}^{2 b} P_{0}^{(2-a-b)}}{P_{\mathrm{g}}^{2}(a+b-2)}\left[1-\left(1+\frac{T_{\mathrm{g}}}{\tau_{0}}\right)^{(2-a-b) /(n-1)}\right]
$$

for $a \neq 2, n \neq 1$, and

$$
\left\langle L_{\gamma} \mid B_{12}\right\rangle \approx \frac{10^{15} c B_{12}^{2(b-1)} P_{0}^{2-a-b}}{T_{\mathrm{g}}(a+b-2)}
$$

for $a>2$ and $n=3$ (refer to Equations (7) and (8) in MC00). In these equations, the initial spin-down time $\tau_{0}$ and the period of the oldest pulsar in the Galaxy $P_{\mathrm{g}}$ can be given by

$$
\begin{aligned}
\tau_{0} & =\frac{10^{15} P_{0}^{2}}{B_{12}^{2}(n-1)} \\
P_{\mathrm{g}} & =P_{0}\left(1+\frac{T_{\mathrm{g}}}{\tau_{0}}\right)^{1 /(n-1)}
\end{aligned}
$$

where $P_{0}$ and $T_{\mathrm{g}}$ are the initial spin period of the pulsar and the age of the Galaxy $\left(T_{\mathrm{g}}=10^{10} \mathrm{yr}\right)$, respectively. 
For a Galactic pulsar population, the number of pulsars for a given flux $G$ can be written as follows

$$
\Delta N_{\mathrm{psr}}(G)=2 G \sinh (1.15 \Delta \log G) \frac{d N_{\mathrm{psr}}}{d G}
$$

where $\Delta \log G$ is the logarithmic bin size of the flux. The differential number of pulsars with respect to flux can be given as

$$
\frac{d N_{\mathrm{psr}}}{d G}=\frac{\Omega}{4 \pi} N_{\mathrm{psr}} \int d L_{\gamma} f_{\mathrm{L}_{\gamma}}\left(L_{\gamma} \mid B_{12}\right) f_{\mathrm{G}}\left(G \mid L_{\gamma}\right)
$$

where the PDFs $f_{\mathrm{L}_{\gamma}}\left(L_{\gamma} \mid B_{12}\right)$ and $f_{\mathrm{G}}\left(G \mid L_{\gamma}\right)$ are given by

$$
\begin{array}{r}
f_{\mathrm{L}_{\gamma}}\left(L_{\gamma} \mid B_{12}\right)=\frac{2 c^{2 /(a+b)}}{(a+b) P_{\mathrm{g}}^{2}} B_{12}^{4 b /(a+b)} L_{\gamma}^{-(1+2 /(a+b))} \\
f_{\mathrm{G}}\left(G \mid L_{\gamma}\right)=\frac{1}{2}\left(\frac{L_{\gamma}}{\Omega}\right)^{1 / 2} f_{\mathrm{D}}\left(D_{\mathrm{i}, \odot}\right) G^{-3 / 2} .
\end{array}
$$

Here, $f_{\mathrm{D}}\left(D_{\mathrm{i}, \odot}\right)$ is the PDF of the spatial distribution of the Galactic pulsar population as a function of the distance from the Sun $D_{\mathrm{i}, \odot}$. Assuming cylindrical symmetry around the Galactic center, we can write the PDF

$$
f_{\mathrm{D}}\left(D_{\mathrm{i}, \odot}\right)=A \frac{D_{\mathrm{i}, \odot}}{2 \pi d_{\odot}} \iint d r d z \rho(r, z) \frac{1}{r}\left[1-\left(\frac{r^{2}+z^{2}+d_{\odot}^{2}-D_{\mathrm{i}, \odot}^{2}}{2 r d_{\odot}}\right)^{2}\right]^{-1 / 2}
$$

where $\left|r^{2}+z^{2}+d_{\odot}^{2}-D_{\mathrm{i}, \odot}^{2}\right| \leq 2 r d_{\odot}$ for all $r$ and $z$. The normalization constant $A$ can 
be evaluated with the condition that $\int d D_{\mathrm{i}, \odot} f_{\mathrm{D}}\left(D_{\mathrm{i}, \odot}\right)=1$ and the pulsar number density $\rho(r, z)$ is given in Equation (5.7). All the derivations of these equations are given in $\mathrm{MC} 00$. 